\title{
Global Existence and Asymptotic Behaviors For Some Nonlinear Partial Differential Equations.
}

Ismahan Dhaw Binshati

benshati2013@yahoo.com

Follow this and additional works at: https://researchrepository.wvu.edu/etd

Part of the Analysis Commons, Harmonic Analysis and Representation Commons, and the Other Mathematics Commons

\section{Recommended Citation}

Binshati, Ismahan Dhaw, "Global Existence and Asymptotic Behaviors For Some Nonlinear Partial Differential Equations." (2019). Graduate Theses, Dissertations, and Problem Reports. 7434.

https://researchrepository.wvu.edu/etd/7434

This Dissertation is protected by copyright and/or related rights. It has been brought to you by the The Research Repository @ WVU with permission from the rights-holder(s). You are free to use this Dissertation in any way that is permitted by the copyright and related rights legislation that applies to your use. For other uses you must obtain permission from the rights-holder(s) directly, unless additional rights are indicated by a Creative Commons license in the record and/ or on the work itself. This Dissertation has been accepted for inclusion in WVU Graduate Theses, Dissertations, and Problem Reports collection by an authorized administrator of The Research Repository @ WVU.

For more information, please contact researchrepository@mail.wvu.edu. 
Global Existence and Asymptotic Behaviors For Some Nonlinear Partial Differential Equations.

\author{
Ismahan Binshati \\ Dissertation submitted to the \\ Eberly College of Arts and Sciences \\ at West Virginia University \\ in partial fulfillment of the requirements \\ for the degree of
}
Doctor of Philosophy
in
Mathematics

\author{
Harumi Hattori, Ph.D Co-Chair \\ Adrian Tudorascu, Ph.D Co-Chair \\ Adam Halasz, Ph.D \\ Casian Pantea, Ph.D \\ Tudor Stanescu, Ph.D
}

Department of Mathematics

Morgantown, WV

2019

Keywords: [Nonlinear Partial Differential Equations]

Copyright 2019 Ismahan Binshati 


\title{
ABSTRACT \\ Global Existence and Asymptotic Behaviors For Some Nonlinear Partial Differential Equations.
}

\author{
Ismahan Binshati
}

We study global existence and asymptotic behavior of the solutions for two-fluid compressible isentropic Euler-Maxwell equations by the Fourier transform and energy method. We discuss the case when the pressure for two fluids is not identical and we also add the friction between two fluids. In addition, we discuss the rates of decay of $L^{p}-L^{q}$ norms for a linear system. Moreover, we use the result for $L^{p}-L^{q}$ estimates to prove the decay rates for the nonlinear systems. In addition, we prove existence of heteroclinic orbits for the nonlinear Vlasov and the one-dimensional Vlasov-Poisson systems. In the nonlinear Vlasov case with sufficiently regular and periodic potential we show that there is at least one orbit emanating from a maximum point of the potential and at least one terminating at it. The Vlasov-Poisson case is more delicate due to the singularity of the potential, and our existence result is limited to one spatial dimension and to weak solutions in the space of probability measures on the torus endowed with the periodic Wasserstein distance. 


\section{Acknowledgements}

We would like to acknwledge support from the referees. 


\section{Contents}

I Global Existence And Asymptotic Behavior Of Solutions For Compressible Two Fluids Euler Maxwell Equation 1

1 Introduction 2

2 Global solution for the nonlinear system 5

2.1 Reformulation of the problem . . . . . . . . . . . 5

2.2 A priori estimates . . . . . . . . . . . . . . 8

2.3 Proof of global existence . . . . . . . . . . . . . . . 19

3 Linearized Homogeneous System 21

3.1 Linearized equations . . . . . . . . . . . . . . . . . 21

3.2 Representation of solution . . . . . . . . . . . . . . . . 27

$3.2 .1 \quad$ Parallel Part . . . . . . . . . . . . . . . . . . . . . . 28

3.2 .2 Perpendicular Part . . . . . . . . . . . . . . . 33

3.2.3 Combine the Parallel Part and Perpendicular Part . . . . . . 45

3.3 Refined $L^{p}-L^{q}$ time-decay property $\ldots \ldots \ldots \ldots \ldots$. . . . 46

4 The decay rates for nonlinear systems.

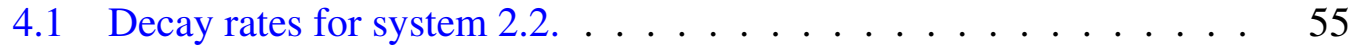

4.2 Time rate for the high-order energy functional . . . . . . . . . 59

4.3 Decay rate in $L^{q} \ldots \ldots \ldots \ldots \ldots \ldots \ldots$

II Heteroclinic Orbits For The nonlinear Vlasov And The onedimensional Vlasov-Poisson Systems 65

5 Introduction $\quad \mathbf{6 6}$

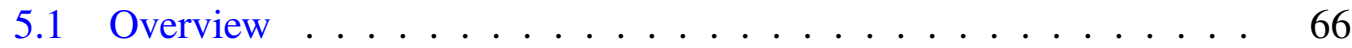

5.2 Main results . . . . . . . . . . . . . . . 70

6 Heteroclinic orbits for the Nonlinear Vlasov system 73 
7 Heteroclinic orbits for the one-dimensional, periodic Vlasov-Poisson system

7.1 Barycentric projection; a periodic Vlasov-Poisson equation . . . . . 82

7.2 Five kinds of trajectories. Ill-posedness for the initial-value problem. Periodic, homoclinic and heteroclinic orbits. . . . . . . . . . 85

8 Appendix 90

8.1 A new inner-product and its corresponding gradient . . . . . . . . 90

8.2 Optimal Transport on the torus . . . . . . . . . . . . . . . 92 


\section{Part I}

\section{Global Existence And Asymptotic Behavior Of Solutions For Compressible Two Fluids Euler Maxwell Equation}




\section{Chapter 1}

\section{Introduction}

We consider the Cauchy problem for first-order nonlinear two-fluid compressible isentropic Euler-Maxwell equations in three dimensions, written as

$$
\left\{\begin{array}{l}
\partial_{t} n_{ \pm}+\nabla \cdot\left(n_{ \pm} u_{ \pm}\right)=0, \\
\partial_{t} u_{ \pm}+u_{ \pm} . \nabla u_{ \pm}+\frac{1}{n_{ \pm}} \nabla p_{ \pm}\left(n_{ \pm}\right)= \pm\left(E+u_{ \pm} \times B\right)-v_{ \pm} u_{ \pm}-\alpha_{ \pm}\left(u_{ \pm}-u_{\mp}\right), \\
\partial_{t} E-\nabla \times B=n_{-} u_{-}-n_{+} u_{+}, \\
\partial_{t} B+\nabla \times E=0, \\
\nabla . E=n_{+}-n_{-}, \quad \nabla . B=0,
\end{array}\right.
$$

where $n_{ \pm}=n_{ \pm}(t, x) \in \mathbb{R}^{3}$ denotes the density of electrons $\left(n_{-}\right)$and ions $\left(n_{+}\right), u_{ \pm}=$ $u_{ \pm}(t, x) \in \mathbb{R}^{3}$ denotes the velocity of electrons $\left(u_{-}\right)$and ions $\left(u_{+}\right), E=E(t, x) \in \mathbb{R}^{3}$ the electric field, and $B=B(t, x) \in \mathbb{R}^{3}$ the magnetic field for $t>0, x \in \mathbb{R}^{3}$. The initial data are given by

$$
\left.\left[n_{ \pm}, u_{ \pm}, E, B\right]\right|_{t=0}=\left[n_{ \pm 0}, u_{ \pm 0}, E_{0}, B_{0}\right], \quad x \in \mathbb{R}^{3},
$$

with the compatibility conditions

$$
\nabla \cdot E_{0}=n_{+0}-n_{-0}, \quad \nabla . B_{0}=0, \quad x \in \mathbb{R}^{3} .
$$

The Euler-Maxwell system (1.1) is a symmetrizable hyperbolic system for $n>0$ and the initial value problem (1.1), (1.2) has a local smooth solution when the initial data are smooth. The global existence of smooth solutions to the initial boundary value problem has been given in [7] by the compensated compactness method. 
The authors of [1],[10] studied the existence of global smooth solutions for the three-dimensional isentropic Euler-Maxwell system with small amplitude and the periodic problem is discussed by Ueda [8]. For the special case where the solution to the Euler-Maxwell equation has asymptotic limits with small parameters, see [11],[12]. The special case of the diffusive relaxation limit of the three-dimenisonal non-isentropic Euler-Maxwell equation is considered in [13],[15]. Two hierarchies of models of the ionospheric plasma for two-fluid Euler-Maxwell equations were presented in [3]. The Fourier transform method was considered by Duan [1],[2] and Ueda [9]. Jerome [14] adapted the classical semigroup-resolvent approach of Kato [5] to Cauchy problem in $\mathbb{R}^{3}$ and established a local smooth solution. In [1] the Duan considered the case when the pressure functions $p_{ \pm}(\cdot)$ depend only on density, having the expression $p_{ \pm}\left(n_{ \pm}\right)=A_{ \pm} n_{ \pm}^{\gamma}$ with constants $A_{+}=A_{-}>0$ and the adiabatic exponent $\gamma>1$.

In this paper, we consider the global existence of smooth solutions for two-fluid compressible isentropic Euler-Maxwell equation extending the results of Duan [1]. In contrast to Duan, we suppose $A_{+} \neq A_{-}$, and we add the friction $\alpha_{ \pm}\left(u_{ \pm}-u_{\mp}\right)$ where $\alpha_{+}=\frac{\alpha}{n_{+}}>0, \alpha_{-}=\frac{\alpha}{n_{-}}>0$, and $\alpha$ is the constant. We obtain the decay rates of smooth solutions by the Fourier transform. The main results are stated as follows:

Theorem 1.1. Let $N \geq 4$ and (1.3) hold. There are $\delta_{0}>0, C_{0}$ such that if

$$
\left\|\left[n_{ \pm 0}-1, u_{ \pm 0}, E_{0}, B_{0}\right]\right\|_{N} \leq \delta_{0}
$$

then the Cauchy problem (1.1)-(1.2) of the Euler-Maxwell system admits a unique global solution $\left[n_{ \pm}(t, x), u_{ \pm}(t, x), E(t, x), B(t, x)\right]$ with

$$
\left[n_{ \pm}(t, x)-1, u_{ \pm}(t, x), E(t, x), B(t, x)\right] \in C\left([0, \infty) ; H^{N}\left(\mathbb{R}^{3}\right)\right) \cap \operatorname{Lip}\left([0, \infty) ; H^{N-1}\left(\mathbb{R}^{3}\right)\right)
$$

and

$$
\sup _{t \geq 0}\left\|\left[n_{ \pm}(t)-1, u_{ \pm}(t), E(t), B(t)\right]\right\|_{N} \leq C_{0}\left\|\left[n_{ \pm 0}-1, u_{ \pm 0}, E_{0}, B_{0}\right]\right\|_{N} .
$$

Moreover, there exist $\delta_{2}>0, C_{1}$ such that if

$$
\left\|\left[n_{ \pm 0}-1, u_{ \pm 0}, E_{0}, B_{0}\right]\right\|_{13}+\left\|\left[n_{ \pm 0}-1, u_{ \pm 0}, E_{0}, B_{0}\right]\right\|_{L^{1}} \leq \delta_{1}
$$

then the solution $\left[n_{ \pm}(t, x), u_{ \pm}(t, x), E(t, x), B(t, x)\right]$ satisfies that for any $t \geq 0$

$$
\begin{gathered}
\left\|n_{ \pm}(t)\right\|_{L^{q}} \leq C_{1}(1+t)^{-\frac{11}{4}} \\
\left\|u_{ \pm}(t), E(t)\right\|_{L^{q}} \leq C_{1}(1+t)^{-2+\frac{3}{2 q}} \\
\|B(t)\|_{L^{q}} \leq C_{1}(1+t)^{-\frac{3}{2}+\frac{3}{2 q}}
\end{gathered}
$$


with $2 \leq q \leq \infty$. Forthermore,

$$
\left\|U_{ \pm}(t)-G(t) * U_{ \pm 0}\right\|_{L^{p}} \leq C \varepsilon_{13}\left(V_{0}\right)(1+t)^{-2+\frac{3}{2 q}}
$$

where $G(t, x)$ is Green's matrix for the linearized system.

The proof of Theorem 1.1 is based on the energy method, and the Fourier transform, as in [1]. There are three key steps: the first key is the priori estimate to establish the global solution and has the form

$$
\mathcal{E}_{N}(V(t))+\int_{0}^{t} \mathcal{D}_{N}(V(s)) d s \leq \mathcal{E}_{N}\left(V_{0}\right),
$$

where $V(t)$ is the perturbation of solution (1.1) and $\varepsilon_{N}(),. \mathcal{D}_{N}($.$) denote the energy$ functional and energy dissipation rate functional as in [1]. This differs from [2] and [8] because the two fluids system has a more complex structure than one fluid, so obtaining energy estimates for the density, the velocity and electric magnetic fields for Euler-Maxwell, requires a different strategy. The time-decay property of solutions to the nonlinear system requires the construction of functionals capturing the optimal energy dissipation rate. The second key step is linearizing the homogeneous form of (1.1) and using the Fourier transform to obtain the $L^{p}-L^{q}$ time decay rate and the explicit representation of the solution. The third step is combining the previous two steps and applying the Fourier transform to obtain the time decay rate of solution to the reformulated non-linear system to finish the proof of Theorem 1.1. Thus the solutions can be represented by the solution of the linearized system and the refined energy estimates using the Duhamel's principle.

We introduce some notations that we will use later in this paper. For any integer $N \geq 0, H^{N}, \dot{H}^{N}$ denote the Sobolev space $H^{N}\left(\mathbb{R}^{3}\right)$ and the $N^{t h}$-order homogeneous Sobolev space, respectively. Set $L^{2}=H^{0}$. The norm of $H^{N}$ is denoted by $\|\cdot\|_{N}$ with $\|\cdot\|=\|\cdot\|_{0} \cdot C$ denotes some positive constant. The inner product in $L^{2}\left(\mathbb{R}^{3}\right)$ denoted by $\langle\cdot, \cdot\rangle$, i.e.,

$$
\langle f, g\rangle=\int_{\mathbb{R}^{3}} f(x) g(x) d x, f=f(x), g=g(x) \in L^{2}\left(\mathbb{R}^{3}\right) .
$$

We denote $\partial^{\alpha}=\partial_{x_{1}}^{\alpha_{1}} \partial_{x_{2}}^{\alpha_{2}} \partial_{x_{3}}^{\alpha_{3}}=\partial_{1}^{\alpha_{1}} \partial_{2}^{\alpha_{2}} \partial_{3}^{\alpha_{3}}$ for multi-index $\alpha=\left[\alpha_{1}, \alpha_{2}, \alpha_{3}\right]$ and the length of $\alpha$ is $|\alpha|=\alpha_{1}+\alpha_{2}+\alpha_{3}$. In addition, $C$ and $\lambda$ denote some positive constants, where both $C$ and $\lambda$ may take different values in different places.

We organize this paper as follows. In section 2 , we reformulate the Cauchy problem and consider the proof of global existence and uniqueness of solutions. From this, we obtain the linearized system for $9 \times 9$. In section 3 , we discuss the time rate of decay for linearized systems. Finally, in section 4, we discuss the time decay rate of solutions of the non-linear system (2.2) and complete the proof of Theorem 1.1. 


\section{Chapter 2}

\section{Global solution for the nonlinear system}

\subsection{Reformulation of the problem}

Denote by $\left[n_{ \pm}, u_{ \pm}, E, B\right]$ a smooth solution to system (1.1) with initial data (1.2) satisfying (1.3). Let

$$
\left\{\begin{array}{l}
\sigma_{ \pm}(t, x)=\sqrt{A_{ \pm}} \frac{2}{\gamma-1}\left(\left[n_{ \pm}\left(\frac{t}{\sqrt{\gamma}}, x\right)\right]^{\frac{\gamma-1}{2}}-1\right), v_{ \pm}=\frac{1}{\sqrt{\gamma}} u_{ \pm}\left(\frac{t}{\sqrt{\gamma}}, x\right), \\
\tilde{E}=\frac{1}{\sqrt{\gamma}} E\left(\frac{t}{\sqrt{\gamma}}, x\right), \tilde{B}=\frac{1}{\sqrt{\gamma}} B\left(\frac{t}{\sqrt{\gamma}}, x\right) .
\end{array}\right.
$$

Define $V:=\left[\sigma_{ \pm}, v_{ \pm}, \tilde{E}, \tilde{B}\right]$ and

$$
\phi\left(\sigma_{ \pm}\right):=\left(\frac{\gamma-1}{2 \sqrt{A_{ \pm}}} \sigma_{ \pm}+1\right)^{\frac{2}{\gamma-1}}-\frac{\sigma_{ \pm}}{\sqrt{A_{ \pm}}}-1 .
$$

Note that $V$ satisfies

$$
\left\{\begin{array}{l}
\partial_{t} \sigma_{ \pm}+\sqrt{A_{ \pm}}\left(\frac{\gamma-1}{2 \sqrt{A_{ \pm}}} \sigma_{ \pm}+1\right) \nabla \cdot v_{ \pm}+v_{ \pm} . \nabla \sigma_{ \pm}=0, \\
\partial_{t} v_{ \pm}+v_{ \pm} . \nabla v_{ \pm}+\sqrt{A_{ \pm}}\left(\frac{\gamma-1}{2 \sqrt{A_{ \pm}}} \sigma_{ \pm}+1\right) \nabla \sigma_{ \pm}= \pm\left(\frac{1}{\sqrt{\gamma}} \tilde{E}+v_{ \pm} \times \tilde{B}\right) \\
\quad-\frac{1}{\sqrt{\gamma}} v_{ \pm} v_{ \pm}-\frac{1}{\sqrt{\gamma}} \alpha_{ \pm}\left(v_{ \pm}-v_{\mp}\right), \\
\partial_{t} \tilde{E}-\frac{1}{\sqrt{\gamma}} \nabla \times \tilde{B}=\frac{1}{\sqrt{\gamma}} v_{-}+\frac{1}{\sqrt{\gamma}}\left[\phi\left(\sigma_{-}\right)+\frac{\sigma_{-}}{\sqrt{A_{-}}}\right] v_{-}-\frac{1}{\sqrt{\gamma}} v_{+}-\frac{1}{\sqrt{\gamma}}\left[\phi\left(\sigma_{+}\right)+\frac{\sigma_{+}}{\sqrt{A_{+}}}\right] v_{+}, \\
\partial_{t} \tilde{B}-\frac{1}{\sqrt{\gamma}} \nabla \times \tilde{E}=0, \\
\nabla . \tilde{E}=\frac{-1}{\sqrt{\gamma}}\left[\phi\left(\sigma_{-}\right)+\frac{\sigma_{-}}{\sqrt{A_{-}}}\right]+\frac{1}{\sqrt{\gamma}}\left[\phi\left(\sigma_{+}\right)+\frac{\sigma_{+}}{\sqrt{A_{+}}}\right], \quad \nabla . \tilde{B}=0, t>0, x \in \mathbb{R}^{3},
\end{array}\right.
$$


with initial data

$$
\left.V\right|_{t=0}=V_{0}:=\left[\sigma_{ \pm 0}, v_{ \pm 0}, \tilde{E_{0}}, \tilde{B_{0}}\right], \quad x \in \mathbb{R}^{3}
$$

Here we have used the notation $V_{0}=\left[\sigma_{ \pm 0}, v_{ \pm 0}, \tilde{E_{0}}, \tilde{B_{0}}\right]$ for the special case where $\left[n_{ \pm 0}, u_{ \pm 0}, E_{0}, B_{0}\right]$ is substituted into (2.1). Note that $V_{0}$ satisfies

$$
\left\{\begin{array}{l}
\nabla \cdot \tilde{E_{0}}=\frac{-1}{\sqrt{\gamma}}\left[\phi\left(\sigma_{+0}\right)+\frac{\sigma_{+0}}{\sqrt{A_{+}}}\right]+\frac{1}{\sqrt{\gamma}}\left[\phi\left(\sigma_{-0}\right)+\frac{\sigma_{-0}}{\sqrt{A_{-}}}\right], \\
\nabla \cdot \tilde{B_{0}}=0, \quad x \in \mathbb{R}^{3} .
\end{array}\right.
$$

Suppose $U:=\left[n_{ \pm}, u_{ \pm}, E, B\right]$ is smooth solution to the intial value proplem of the original Cauchy problem (1.1), (1.2), which satisfies (1.3). Now, we introduce the another transformation by setting $\rho_{ \pm}(t, x)=n_{ \pm}(t, x)-1$, then $U:=\left[\rho_{ \pm}, u_{ \pm}, E, B\right]$ satisfies

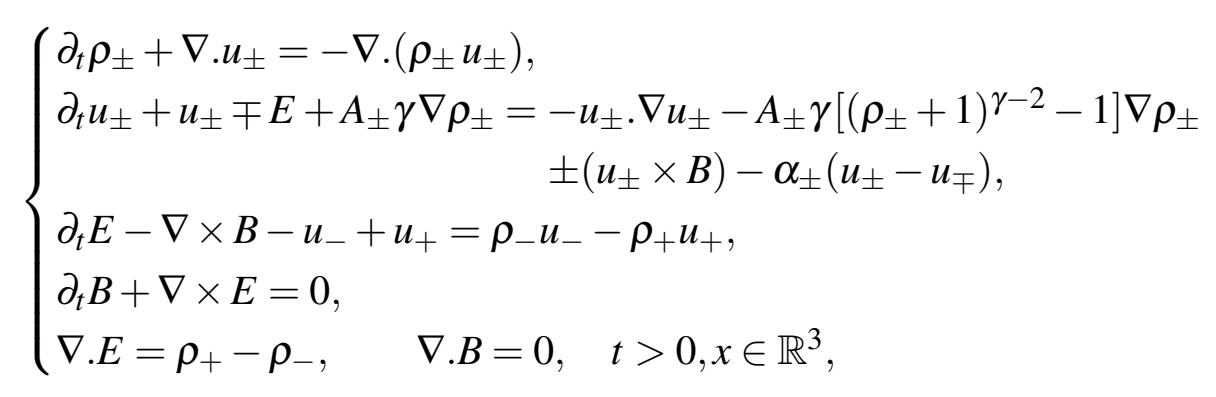

with initial data

$$
\left.U\right|_{t}=U_{0}:=\left[\rho_{ \pm 0}, u_{ \pm 0}, E_{0}, B_{0}\right], \quad x \in \mathbb{R}^{3},
$$

satisfying the compatibility condition

$$
\nabla \cdot E_{0}=\rho_{+0}-\rho_{-0}, \quad \nabla \cdot B_{0}=0,
$$

where $\rho_{ \pm 0}=n_{ \pm 0}-1$.

We will assume $N \geq 4$ is an integer. In addition to $V=\left[\sigma_{ \pm}, v_{ \pm}, \tilde{E}, \tilde{B}\right]$, define the full instant energy functional $\mathcal{E}_{N}(V(t))$ and the high-order instant energy functional $\mathcal{E}_{N}^{h}(V(t))$ via

$$
\left\{\begin{aligned}
\mathcal{E}_{N}(V(t))= & \|V(t)\|_{N}^{2}+k_{1} \sum_{|\alpha| \leq N-1}\left(\left\langle\partial^{\alpha} v_{+}, \nabla \partial^{\alpha} \sigma_{+}\right\rangle+\left\langle\partial^{\alpha} v_{-}, \nabla \partial^{\alpha} \sigma_{-}\right\rangle\right) \\
& +k_{2} \sum_{|\alpha| \leq N-1}\left\langle\partial^{\alpha}\left(v_{+}-v_{-}\right), \nabla \partial^{\alpha} \tilde{E}\right\rangle-k_{3} \sum_{|\alpha| \leq N-2}\left\langle\nabla \times \partial^{\alpha} \tilde{E}, \partial^{\alpha} \tilde{B}\right\rangle
\end{aligned}\right.
$$


and

$$
\left\{\begin{aligned}
\varepsilon_{N}^{h}(V(t))= & \|\nabla V(t)\|_{N-1}^{2}+k_{1} \sum_{1 \leq|\alpha| \leq N-1}\left(\left\langle\partial^{\alpha} v_{+}, \nabla \partial^{\alpha} \sigma_{+}\right\rangle\right. \\
& \left.+\left\langle\partial^{\alpha} v_{-}, \nabla \partial^{\alpha} \sigma_{-}\right\rangle\right)+k_{2} \sum_{1 \leq|\alpha| \leq N-1}\left\langle\partial^{\alpha}\left(v_{+}-v_{-}\right), \nabla \partial^{\alpha} \tilde{E}\right\rangle \\
& -k_{3} \sum_{1 \leq|\alpha| \leq N-2}\left\langle\nabla \times \partial^{\alpha} \tilde{E}, \partial^{\alpha} \tilde{B}\right\rangle,
\end{aligned}\right.
$$

where $0<k_{3} \ll k_{2} \ll k_{1} \ll 1$ are constants to be chosen later in the proof such that $k_{i},(i=1,2,3)$ are small enough and satisfy

$$
\begin{gathered}
\mathcal{E}_{N}(V(t)) \sim\left\|\left[\sigma_{ \pm}, v_{ \pm}, \tilde{E}, \tilde{B}\right]\right\|_{N}^{2}, \\
\mathcal{E}_{N}^{h}(V(t)) \sim\left\|\nabla\left[\sigma_{ \pm}, v_{ \pm}, \tilde{E}, \tilde{B}\right]\right\|_{N-1}^{2} .
\end{gathered}
$$

Define the dissipation rates $\mathcal{D}_{N}(V(t)), \mathcal{D}_{N}^{h}(V(t))$ by

$$
\left\{\begin{aligned}
\mathcal{D}_{N}(V(t))= & \left\|\left[v_{+}, v_{-}\right]\right\|_{N}^{2}+\left\|\nabla\left[\sigma_{+}, \sigma_{-}\right]\right\|_{N-1}^{2}+\|\nabla[\tilde{E}, \tilde{B}]\|_{N-2}^{2} \\
& +\|\tilde{E}\|^{2}+\left\|\sigma_{+}-\sigma_{-}\right\|^{2}
\end{aligned}\right.
$$

and

$$
\left\{\begin{array}{c}
\mathcal{D}_{N}^{h}(V(t))= \\
+\nabla \nabla\left[v_{+}, v_{-}\right]\left\|_{N-1}^{2}+\right\| \nabla^{2}\left[\sigma_{+}, \sigma_{-}\right]\left\|_{N-2}^{2}+\right\| \nabla^{2}[\tilde{E}, \tilde{B}] \|_{N-3}^{2} \\
+\|\nabla \tilde{E}\|^{2}+\left\|\nabla\left(\sigma_{+}-\sigma_{-}\right)\right\|^{2} .
\end{array}\right.
$$

Proposition 2.1. Suppose initial data $V_{0}=\left[\sigma_{ \pm 0}, v_{ \pm 0}, \tilde{E_{0}}, \tilde{B_{0}}\right]$ satisfies (2.4). Then there exist $\mathcal{E}_{N}(\cdot)$ and $\mathcal{D}_{N}(\cdot)$ having the form (2.8) and (2.12), respectively, such that if $\mathcal{E}_{N}\left(V_{0}\right)>0$ is sufficently small, the Cauchy problem (2.2), (2.3) admits a unique, global, non-zero solution $V=\left[\sigma_{ \pm}, v_{ \pm}, \tilde{E}, \tilde{B}\right]$ satisfying

$$
V \in C\left([0, \infty) ; H^{N}\left(\mathbb{R}^{3}\right)\right) \cap \operatorname{Lip}\left([0, \infty) ; H^{N-1}\left(\mathbb{R}^{3}\right)\right),
$$

and

$$
\mathcal{E}_{N}(V(t))+\lambda \int_{0}^{t} \mathcal{D}_{N}(V(s)) d s \leq \mathcal{E}_{N}\left(V_{0}\right)
$$

for any $t \geq 0$.

Remark 2.1. The solutions obtained in Proposition 2.1 indeed the decay rates in time under some reguarity and integrablity conditions on initial data $V_{0}=\left[\sigma_{ \pm 0}, V_{ \pm 0}, \tilde{E_{0}}, \tilde{B_{0}}\right]$ and set

$$
\varepsilon_{m}\left(V_{0}\right)=\left\|V_{0}\right\|_{m}+\left\|\left[v_{ \pm 0}, \tilde{E_{0}}, \tilde{B_{0}}\right]\right\|_{L^{1}},
$$

for the integer $m \geq 4$.

Remark 2.2. Note that the existence result in Theorem 1.1 follows from Proposition 2.1, the derivation of rates of (1.4), (1.6) in Theorem 1.1, and Proposition 4.1. The proof of Proposition 4.1 is analogous to that of Lemmas 5.2 in [2] 


\subsection{A priori estimates}

In this subsection, we obtain uniform-in-time a priori estimates for smooth solutions to the Cauchy problem (2.2), (2.3) by using the classical energy method.

Theorem 2.1. Let $0<T \leq \infty$ be given. Suppose that $V=\left[\sigma_{ \pm}, v_{ \pm}, \tilde{E}, \tilde{B}\right] \in C\left([0, T) ; H^{N}\left(\mathbb{R}^{3}\right)\right)$ is smooth, that $\sigma$ satisfies

$$
\sup _{0 \leq t \leq T}\left\|\sigma_{ \pm}(t)\right\|_{N} \leq 1
$$

and that $V$ solves the system (2.2) for $t \in(0, T)$. Then there exist $\mathcal{E}_{N}($.$) and \mathcal{D}_{N}($. having the form (2.8) and (2.12) such that for all $0 \leq t \leq T$

$$
\frac{d}{d t} \varepsilon_{N}(V(t))+\lambda \mathcal{D}_{N}(V(t)) \leq C\left[\mathcal{E}_{N}(V(t))^{1 / 2}+\mathcal{E}_{N}(V(t))\right] \mathcal{D}_{N}(V(t)) .
$$

Proof: The proof is divided into five steps as follows.

$\operatorname{Step}(1)$ We want to show

$$
\frac{1}{2} \frac{d}{d t}\|V\|_{N}^{2}+\frac{1}{\sqrt{\gamma}} v_{ \pm}\left\|\left[v_{+}, v_{-}\right]\right\|_{N}^{2} \leq C\|V\|_{N}\left(\left\|\left[v_{+}, v_{-}\right]\right\|^{2}+\left\|\nabla\left[\sigma_{+}, \sigma_{-}, v_{+}, v_{-}\right]\right\|_{N-1}^{2}\right) .
$$

Apply $\partial^{\alpha}$ to the first equation of (2.2), then multiply that equation by $\partial^{\alpha} \sigma_{ \pm}$:

$$
\partial_{t} \partial^{\alpha} \sigma_{ \pm}+\partial^{\alpha}\left(v_{ \pm} \cdot \nabla \sigma_{ \pm}\right)+\partial^{\alpha}\left[\sqrt{A_{ \pm}}\left(\frac{\gamma-1}{2 \sqrt{A_{ \pm}}} \sigma_{ \pm}+1\right) \nabla \cdot v_{ \pm}\right]=0
$$

Then,

$$
\begin{aligned}
\frac{1}{2} \frac{d}{d t}\left\|\partial^{\alpha} \sigma_{ \pm}\right\|^{2} & +\sum_{\beta<\alpha} C_{\beta}^{\alpha}\left\langle\partial^{\alpha-\beta} v_{ \pm} \cdot \nabla \partial^{\beta} \sigma_{ \pm}, \partial^{\alpha} \sigma_{ \pm}\right\rangle+\left\langle\partial^{\alpha} v_{ \pm} \cdot \nabla \sigma_{ \pm}, \partial^{\alpha} \sigma_{ \pm}\right\rangle \\
& +\frac{\gamma-1}{2} \sum_{\beta<\alpha} C_{\beta}^{\alpha}\left\langle\partial^{\alpha-\beta} \sigma_{ \pm} \nabla \cdot \partial^{\beta} v_{ \pm}, \partial^{\alpha} \sigma_{ \pm}\right\rangle=0 .
\end{aligned}
$$

Apply $\partial^{\alpha}$ to the second equation of (2.2), then multiply that equation by $\partial^{\alpha} v_{ \pm}$:

$$
\begin{aligned}
\partial_{t} \partial^{\alpha} v_{ \pm} & +\partial^{\alpha}\left[\sqrt{A_{ \pm}}\left(\frac{\gamma-1}{2 \sqrt{A_{ \pm}}} \sigma_{ \pm}+1\right) \nabla \sigma_{ \pm}\right]+\partial^{\alpha}\left(v_{ \pm} \cdot \nabla v_{ \pm}\right) \\
& =\partial^{\alpha}\left[ \pm\left(\frac{1}{\sqrt{\gamma}} \tilde{E}+v_{ \pm} \times \tilde{B}\right)\right]-\frac{1}{\sqrt{\gamma}} v_{ \pm} \partial^{\alpha} v_{ \pm}-\frac{1}{\sqrt{\gamma}} \alpha_{ \pm} \partial^{\alpha}\left(v_{ \pm}-v_{\mp}\right)
\end{aligned}
$$


Then,

$$
\begin{aligned}
& \frac{1}{2} \frac{d}{d t}\left\|\partial^{\alpha} v_{ \pm}\right\|^{2}+\frac{\gamma-1}{2} \sum_{\beta<\alpha} C_{\beta}^{\alpha}\left\langle\partial^{\alpha-\beta} \sigma_{ \pm} \cdot \nabla \partial^{\beta} \sigma_{ \pm}, \partial^{\alpha} v_{ \pm}\right\rangle \\
& +\frac{\gamma-1}{2}\left\langle\partial^{\alpha} \sigma_{ \pm} \cdot \nabla \sigma_{ \pm}, \partial^{\alpha} v_{ \pm}\right\rangle+\sum_{\beta<\alpha} C_{\beta}^{\alpha}\left\langle\partial^{\alpha-\beta} v_{ \pm} \cdot \nabla \partial^{\beta} v_{ \pm}, \partial^{\alpha} v_{ \pm}\right\rangle \\
& +\left\langle\partial^{\alpha} v_{ \pm} \cdot \nabla v_{ \pm}, \partial^{\alpha} v_{ \pm}\right\rangle= \pm \frac{1}{\sqrt{\gamma}}\left\langle\partial^{\alpha} \tilde{E}, \partial^{\alpha} v_{ \pm}\right\rangle \\
& \pm\left\langle v_{ \pm} \times \partial^{\alpha} \tilde{B}, \partial^{\alpha} v_{ \pm}\right\rangle \pm \sum_{\beta<\alpha} C_{\beta}^{\alpha}\left\langle\partial^{\alpha-\beta} v_{ \pm} \times \partial^{\beta} \tilde{B}, \partial^{\alpha} v_{ \pm}\right\rangle \\
& -\frac{1}{\sqrt{\gamma}} v_{ \pm}\left\langle\partial^{\alpha} v_{ \pm}, \partial^{\alpha} v_{ \pm}\right\rangle-\frac{1}{\sqrt{\gamma}} \alpha_{ \pm}\left\langle\partial^{\alpha}\left(v_{ \pm}-v_{\mp}\right), \partial^{\alpha} v_{\mp}\right\rangle .
\end{aligned}
$$

Next, taking the sum of equations (2.20) and (2.21) we find

$$
\begin{aligned}
& \frac{1}{2} \frac{d}{d t}\left\|\partial^{\alpha}\left[\sigma_{+}, \sigma_{-}, v_{+}, v_{-}\right]\right\|^{2}+\frac{1}{\sqrt{\gamma}} v_{ \pm}\left\|\partial^{\alpha}\left[v_{+}, v_{-}\right]\right\|-\frac{1}{\sqrt{\gamma}}\left\langle\partial^{\alpha} \tilde{E}, \partial^{\alpha} v_{+}\right\rangle \\
& +\frac{1}{\sqrt{\gamma}}\left\langle\partial^{\alpha} \tilde{E}, \partial^{\alpha} v_{-}\right\rangle+\frac{1}{\sqrt{\gamma}} \alpha_{ \pm}\left\langle\partial^{\alpha}\left(v_{ \pm}-v_{\mp}\right), \partial^{\alpha} v_{\mp}\right\rangle \\
& =-\sum_{\beta<\alpha} C_{\beta}^{\alpha}\left\langle\partial^{\alpha-\beta} v_{ \pm} \cdot \nabla \partial^{\beta} \sigma_{ \pm}, \partial^{\alpha} \sigma_{ \pm}\right\rangle-\frac{\gamma-1}{2} \sum_{\beta<\alpha} C_{\beta}^{\alpha}\left\langle\partial^{\alpha-\beta} \sigma_{ \pm} \nabla \cdot \partial^{\beta} v_{ \pm}, \partial^{\alpha} \sigma_{ \pm}\right\rangle \\
& -\frac{\gamma-1}{2} \sum_{\beta<\alpha} C_{\beta}^{\alpha}\left\langle\partial^{\alpha-\beta} \sigma_{ \pm} \cdot \nabla \partial^{\beta} \sigma_{ \pm}, \partial^{\alpha} v_{ \pm}\right\rangle \\
& -\sum_{\beta<\alpha} C_{\beta}^{\alpha}\left\langle\partial^{\alpha-\beta} v_{ \pm} \cdot \nabla \partial^{\beta} v_{ \pm}, \partial^{\alpha} v_{ \pm}\right\rangle \pm \sum_{\beta<\alpha} C_{\beta}^{\alpha}\left\langle\partial^{\alpha-\beta} v_{ \pm} \times \partial^{\beta} \tilde{B}, \partial^{\alpha} v_{ \pm}\right\rangle \\
& \pm\left\langle v_{ \pm} \times \partial^{\alpha} \tilde{B}, \partial^{\alpha} v_{ \pm}\right\rangle-\frac{\gamma-1}{2}\left\langle\partial^{\alpha} \sigma_{ \pm} \cdot \nabla \sigma_{ \pm}, \partial^{\alpha} v_{ \pm}\right\rangle-\frac{1}{2}\left\langle\nabla \cdot v_{ \pm},\left|\partial^{\alpha} \sigma_{ \pm}\right|^{2}+\left|\partial^{\alpha} v_{ \pm}\right|^{2}\right\rangle,
\end{aligned}
$$


where

$$
\begin{aligned}
I_{\alpha \beta}^{+}(t) & =\left\langle\partial^{\alpha-\beta} v_{+} \cdot \nabla \partial^{\beta} \sigma_{+}, \partial^{\alpha} \sigma_{+}\right\rangle+\frac{\gamma-1}{2}\left\langle\partial^{\alpha-\beta} \sigma_{+} \nabla \cdot \partial^{\beta} v_{+}, \partial^{\alpha} \sigma_{+}\right\rangle \\
& -\frac{\gamma-1}{2}\left\langle\partial^{\alpha-\beta} \sigma_{+} \cdot \nabla \partial^{\beta} \sigma_{+}, \partial^{\alpha} v_{+}\right\rangle-\left\langle\partial^{\alpha-\beta} v_{+} \cdot \nabla \partial^{\beta} v_{+}, \partial^{\alpha} v_{+}\right\rangle \\
& +\left\langle\partial^{\alpha-\beta} v_{+} \times \partial^{\beta} \tilde{B}, \partial^{\alpha} v_{+}\right\rangle \\
I_{\alpha \beta}^{-}(t) & =\left\langle\partial^{\alpha-\beta} v_{-} \cdot \nabla \partial^{\beta} \sigma_{-}, \partial^{\alpha} \sigma_{-}\right\rangle+\frac{\gamma-1}{2}\left\langle\partial^{\alpha-\beta} \sigma_{-} \nabla \cdot \partial^{\beta} v_{-}, \partial^{\alpha} \sigma_{-}\right\rangle \\
& -\frac{\gamma-1}{2}\left\langle\partial^{\alpha-\beta} \sigma_{-} \nabla \partial^{\beta} \sigma_{-}, \partial^{\alpha} v_{-}\right\rangle-\left\langle\partial^{\alpha-\beta} v_{-} \cdot \nabla \partial^{\beta} v_{-}, \partial^{\alpha} v_{-}\right\rangle \\
& -\left\langle\partial^{\alpha-\beta} v_{-} \times \partial^{\beta} \tilde{B}, \partial^{\alpha} v_{-}\right\rangle \\
I_{\alpha \beta}(t) & =I_{\alpha \beta}^{+}(t)+I_{\alpha \beta}^{-}(t)
\end{aligned}
$$

Note that we used integration by parts. When $|\alpha|=0$ it suffices to estimate $I_{+}(t)$ and $I_{-}(t)$ by

$$
\begin{aligned}
I_{1}^{+}(t)= & \frac{1}{2}\left\langle\nabla \cdot v_{+},\left|\partial^{\alpha} \sigma_{+}\right|^{2}+\left|\partial^{\alpha} v_{+}\right|^{2}\right\rangle+\frac{\gamma-1}{2}\left\langle\partial^{\alpha} \sigma_{+} \cdot \nabla \sigma_{+}, \partial^{\alpha} v_{+}\right\rangle \\
& +\left\langle v_{+} \times \partial^{\alpha} \tilde{B}, \partial^{\alpha} v_{+}\right\rangle+\frac{1}{\sqrt{\gamma}} \alpha_{+}\left\langle\partial^{\alpha}\left(v_{+}-v_{-}\right), \partial^{\alpha} v_{+}\right\rangle \\
I_{1}^{-}(t) & =\frac{1}{2}\left\langle\nabla \cdot v_{-},\left|\partial^{\alpha} \sigma_{-}\right|^{2}+\left|\partial^{\alpha} v_{-}\right|^{2}\right\rangle+\frac{\gamma-1}{2}\left\langle\partial^{\alpha} \sigma_{-} \cdot \nabla \sigma_{-}, \partial^{\alpha} v_{-}\right\rangle \\
& -\left\langle v_{-} \times \partial^{\alpha} \tilde{B}, \partial^{\alpha} v_{-}\right\rangle+\frac{1}{\sqrt{\gamma}} \alpha_{-}\left\langle\partial^{\alpha}\left(v_{-}-v_{+}\right), \partial^{\alpha} v_{-}\right\rangle
\end{aligned}
$$

where

$$
I_{1}(t)=I_{1}^{+}(t)+I_{1}^{-}(t)
$$

so that

$$
\begin{aligned}
I_{1}(t) & \leq C\left\|\left[\sigma_{+}, v_{+}\right]\right\|_{H^{1}}\left\|\nabla\left[\sigma_{+}, v_{+}\right]\right\|^{2}+C\|\nabla \tilde{B}\|_{H^{1}}\left\|v_{+}\right\|^{2} \\
& +C\left\|\left[\sigma_{-}, v_{-}\right]\right\|_{H^{1}}\left\|\nabla\left[\sigma_{-}, v_{-}\right]\right\|^{2}+C\|\nabla \tilde{B}\|_{H^{1}}\left\|v_{-}\right\|^{2} \\
& \leq C\left\|\left[\sigma_{+}, \sigma_{-}, v_{+}, v_{-}\right]\right\|_{H^{1}}\left\|\nabla\left[\sigma_{+}, \sigma_{-}, v_{+}, v_{-}\right]\right\|^{2}+C\|\nabla \tilde{B}\|_{H^{1}}\left\|v_{+}, v_{-}\right\|^{2} .
\end{aligned}
$$

Furthermore, $I(t)$ is bounded by the right-hand side term of (2.19). When $|\alpha| \geq 1$ each term in $I_{\alpha, \beta}(t)$ and $I_{l}(t)$ is an integral of a product of three terms in which there is at least one term containing the derivative. Thus

$$
I_{\alpha, \beta}(t)+I_{1}(t) \leq C\left\|\left[\sigma_{+}, \sigma_{-}, v_{+}, v_{-}, \tilde{B}\right]\right\|_{N}+\left\|\nabla\left[\sigma_{+}, \sigma_{-}, v_{+}, v_{-}\right]\right\|_{N-1}^{2} .
$$


Now, multiply the third equation of (2.2) by $\partial^{\alpha} E$, with $|\alpha| \leq N$ :

$$
\begin{aligned}
\partial_{t^{\prime}} \partial^{\alpha} \tilde{E}-\frac{1}{\sqrt{\gamma}} \nabla \times \partial^{\alpha} \tilde{B} & =\frac{1}{\sqrt{\gamma}} \partial^{\alpha} v_{-}+\frac{1}{\sqrt{\gamma}} \partial^{\alpha}\left(\left[\phi\left(\sigma_{-}\right)+\frac{\sigma_{-}}{\sqrt{A_{-}}}\right] v_{-}\right) \\
& -\frac{1}{\sqrt{\gamma}} \partial^{\alpha} v_{+}-\frac{1}{\sqrt{\gamma}} \partial^{\alpha}\left(\left[\phi\left(\sigma_{+}\right)-\frac{\sigma_{+}}{\sqrt{A_{+}}}\right] v_{+}\right) .
\end{aligned}
$$

Then

$$
\begin{aligned}
\frac{d}{d t}\left\langle\partial^{\alpha} \tilde{E}, \partial^{\alpha} \tilde{E}\right\rangle & -\frac{1}{\sqrt{\gamma}}\left\langle\nabla \times \partial^{\alpha} \tilde{B}, \partial^{\alpha} \tilde{E}\right\rangle=\frac{1}{\sqrt{\gamma}}\left\langle\partial^{\alpha} v_{-}, \partial^{\alpha} \tilde{E}\right\rangle \\
& +\frac{1}{\sqrt{\gamma}}\left\langle\partial^{\alpha}\left[\phi\left(\sigma_{-}\right) v_{-}+\frac{\sigma_{-}}{\sqrt{A_{-}}} v_{-}\right], \partial^{\alpha} \tilde{E}\right\rangle-\frac{1}{\sqrt{\gamma}}\left\langle\partial^{\alpha} v_{+}, \partial^{\alpha} \tilde{E}\right\rangle \\
& -\frac{1}{\sqrt{\gamma}}\left\langle\partial^{\alpha}\left[\phi\left(\sigma_{+}\right) v_{+}+\frac{\sigma_{+}}{\sqrt{A_{+}}} v_{+}\right], \partial^{\alpha} \tilde{E}\right\rangle
\end{aligned}
$$

Now, multiply the fourth equation of (2.2) by $\partial^{\alpha} \tilde{B}$ with $|\alpha| \leq N$ :

$$
\partial_{t^{\prime}} \partial^{\alpha} \tilde{B}+\frac{1}{\sqrt{\gamma}} \nabla \times \partial^{\alpha} \tilde{E}=0
$$

Then

$$
\frac{d}{d t}\left\langle\partial^{\alpha} \tilde{B}, \partial^{\alpha} \tilde{B}\right\rangle+\frac{1}{\sqrt{\gamma}}\left\langle\nabla \times \partial^{\alpha} \tilde{E}, \partial^{\alpha} \tilde{B}\right\rangle .
$$

Taking the sum of equations (2.27) and (2.28), we find

$$
\begin{gathered}
\frac{1}{2} \frac{d}{d t}\left\|\partial^{\alpha}[\tilde{E}, \tilde{B}]\right\|^{2}-\frac{1}{\sqrt{\gamma}}\left\langle\partial^{\alpha} v_{-}, \partial^{\alpha} \tilde{E}\right\rangle+\frac{1}{\sqrt{\gamma}}\left\langle\partial^{\alpha} v_{+}, \partial^{\alpha} \tilde{E}\right\rangle \\
=+\frac{1}{\sqrt{\gamma}}\left\langle\partial^{\alpha}\left[\phi\left(\sigma_{-}\right) v_{-}+\frac{\sigma_{-}}{\sqrt{A_{-}}} v_{-}\right], \partial^{\alpha} \tilde{E}\right\rangle \\
-\frac{1}{\sqrt{\gamma}}\left\langle\partial^{\alpha}\left[\phi\left(\sigma_{+}\right) v_{+}+\frac{\sigma_{+}}{\sqrt{A_{+}}} v_{+}\right], \partial^{\alpha} \tilde{E}\right\rangle,
\end{gathered}
$$

where

$$
I_{2}^{+}(t)=-\frac{1}{\sqrt{\gamma}}\left\langle\partial^{\alpha}\left[\phi\left(\sigma_{+}\right) v_{+}+\frac{\sigma_{+}}{\sqrt{A_{+}}} v_{+}\right], \partial^{\alpha} \tilde{E}\right\rangle
$$

and

$$
I_{2}^{-}(t)=+\frac{1}{\sqrt{\gamma}}\left\langle\partial^{\alpha}\left[\phi\left(\sigma_{-}\right) v_{-}+\frac{\sigma_{-}}{\sqrt{A_{-}}} v_{-}\right], \partial^{\alpha} \tilde{E}\right\rangle .
$$


Define

$$
I_{2}(t):=I_{2}^{+}(t)+I_{2}^{-}(t) .
$$

In a similar way as [10], for $|\alpha| \leq N$

$$
I_{2}^{+}(t) \leq C\|\tilde{E}\|_{N}\left(\left\|\nabla\left[\sigma_{+}, v_{+}\right]\right\|_{N}^{2}+\left\|v_{+}\right\|^{2}\right)
$$

and

$$
I_{2}^{-}(t) \leq C\|\tilde{E}\|_{N}\left(\left\|\nabla\left[\sigma_{-}, v_{-}\right]\right\|_{N}^{2}+\left\|v_{-}\right\|^{2}\right)
$$

Thus, for $|\alpha| \leq N$

$$
I_{2}^{+}(t)+I_{2}^{-}(t) \leq C\|\tilde{E}\|_{N}\left(\left\|\nabla\left[\sigma_{+}, \sigma_{-}, v_{+}, v_{-}\right]\right\|_{N}^{2}+\left\|\left[v_{+}, v_{-}\right]\right\|^{2}\right),
$$

and we therefore have

$\frac{1}{2} \frac{d}{d t}\|V\|_{N}^{2}+\frac{1}{\sqrt{\gamma}} v_{ \pm}\left\|\partial^{\alpha}\left[v_{+}, v_{-}\right]\right\|_{N}^{2} \leq\|V\|_{N}\left(\left\|\nabla\left[\sigma_{+}, \sigma_{-}, v_{+}, v_{-}\right]\right\|_{N-1}^{2}+\left\|\left[v_{+}, v_{-}\right]\right\|^{2}\right)$

Step (2) We want to show that

$$
\begin{aligned}
& \frac{d}{d t} \mathcal{E}_{N, 1}^{i n t}(V)+\lambda\left(\left\|\nabla\left[\sigma_{+}, \sigma_{-}\right]\right\|_{N-1}^{2}+\left\|\frac{\sigma_{+}}{\sqrt{A_{+}}}-\frac{\sigma_{-}}{\sqrt{A_{-}}}\right\|_{N}^{2}\right) \\
& \quad \leq C\left\|\left[v_{+}, v_{-}\right]\right\|_{N}^{2}+C\left\|\left[\sigma_{+}, \sigma_{-}, v_{+}, v_{-}, \tilde{B}\right]\right\|_{N}^{2}\left\|\nabla\left[\sigma_{+}, \sigma_{-}, v_{+}, v_{-}\right]\right\|_{N-1}^{2}
\end{aligned}
$$

where $\mathcal{E}_{N, 1}^{\text {int }}(\cdot)$ is defined by

$$
\varepsilon_{N, 1}^{i n t}(V)=\sum_{|\alpha| \leq N-1}\left(\left\langle\partial^{\alpha} v_{+}, \nabla \partial^{\alpha} \sigma_{+}\right\rangle+\left\langle\partial^{\alpha} v_{-}, \nabla \partial^{\alpha} \sigma_{-}\right\rangle\right)
$$

First, rewrite the first and second equations of (2.2) by putting the linear terms on the left-hand sides and the nonlinear terms on right-hand sides:

$$
\begin{gathered}
\partial_{t} \sigma_{+}+\sqrt{A_{+}} \nabla \cdot v_{+}=-v_{+} \cdot \nabla \sigma_{+}-\frac{\gamma-1}{2} \sigma_{+} \nabla \cdot v_{+}:=f_{1}^{+} \\
\partial_{t} \sigma_{-}+\sqrt{A_{-}} \nabla \cdot v_{-}=-v_{-} \cdot \nabla \sigma_{-}-\frac{\gamma-1}{2} \sigma_{-} \nabla \cdot v_{-}:=f_{1}^{-} \\
\partial_{t} v_{+}+\sqrt{A_{+}} \nabla \sigma_{+}-\frac{1}{\sqrt{\gamma}} \tilde{E}+\frac{v_{+}}{\sqrt{\gamma}} v_{+}+\frac{\alpha_{+}}{\sqrt{\gamma}}\left(v_{+}-v_{-}\right) \\
=-v_{+} \cdot \nabla v_{+}-\frac{\gamma-1}{2} \sigma_{+} \nabla \sigma_{+}+v_{+} \times \tilde{B}:=f_{2}^{+}
\end{gathered}
$$




$$
\begin{gathered}
\partial_{t} v_{-}+\sqrt{A_{-}} \nabla \sigma_{-}+\frac{1}{\sqrt{\gamma}} \tilde{E}+\frac{v_{-}}{\sqrt{\gamma}} v_{-}+\frac{\alpha_{-}}{\sqrt{\gamma}}\left(v_{-}-v_{+}\right) \\
=-v_{-} \cdot \nabla v_{-}-\frac{\gamma-1}{2} \sigma_{-} \nabla \sigma_{-}-v_{-} \times \tilde{B}:=f_{2}^{-}
\end{gathered}
$$

Let $|\alpha| \leq N-1$. Applying $\partial^{\alpha}$ to (2.33), multiplying by $\nabla \partial^{\alpha} \sigma_{+}$, and integrating in $x$ we find

$$
\begin{aligned}
& \left\langle\nabla \partial^{\alpha} \sigma_{+}, \partial_{t} \partial^{\alpha} v_{+}\right\rangle+\sqrt{A_{+}}\left\langle\nabla \partial^{\alpha} \sigma_{+}, \nabla \partial^{\alpha} \sigma_{+}\right\rangle-\frac{1}{\sqrt{\gamma}}\left\langle\nabla \partial^{\alpha} \sigma_{+}, \partial^{\alpha} \tilde{E}\right\rangle \\
& +\frac{v_{+}}{\sqrt{\gamma}}\left\langle\nabla \partial^{\alpha} \sigma_{+}, \partial^{\alpha} v_{+}\right\rangle+\frac{\alpha_{+}}{\sqrt{\gamma}}\left\langle\nabla \partial^{\alpha} \sigma_{+}, \partial^{\alpha}\left(v_{+}-v_{-}\right)\right\rangle=-\left\langle\nabla \partial^{\alpha} \sigma_{+}, \partial^{\alpha} v_{+} \cdot \nabla v_{+}\right\rangle \\
& -\left\langle\nabla \partial^{\alpha} \sigma_{+}, v_{+} \cdot \nabla \partial^{\alpha} v_{+}\right\rangle-\frac{\gamma-1}{2}\left\langle\nabla \partial^{\alpha} \sigma_{+}, \partial^{\alpha} \sigma_{+} \nabla \sigma_{+}\right\rangle-\frac{\gamma-1}{2}\left\langle\nabla \partial^{\alpha} \sigma_{+}, \sigma_{+} \nabla \partial^{\alpha} \sigma_{+}\right\rangle \\
& +\left\langle\nabla \partial^{\alpha} \sigma_{+}, \partial^{\alpha} v_{+} \times \tilde{B}\right\rangle+\left\langle\nabla \partial^{\alpha} \sigma_{+}, v_{+} \times \partial^{\alpha} \tilde{B}\right\rangle:=f_{2}^{+} .
\end{aligned}
$$

Since

$$
\left\langle\partial_{t} \partial^{\alpha} v_{+}, \nabla \partial^{\alpha} \sigma_{+}\right\rangle=\frac{\partial}{\partial_{t}}\left\langle\nabla \partial^{\alpha} \sigma_{+}, \partial^{\alpha} v_{+}\right\rangle-\left\langle\partial^{\alpha} v_{+}, \nabla \partial^{\alpha} \frac{\partial}{\partial_{t}} \sigma_{+}\right\rangle
$$

if we multiply $(2.35)$ by $\frac{1}{\sqrt{A_{+}}}$, then we obtain

$$
\begin{aligned}
\frac{\partial}{\partial_{t}} & \frac{1}{\sqrt{A_{+}}}\left\langle\nabla \partial^{\alpha} \sigma_{+}, \partial^{\alpha} v_{+}\right\rangle+\left\|\nabla \partial^{\alpha} \sigma_{+}\right\|^{2}-\frac{1}{\gamma}\left\langle\frac{\partial^{\alpha} \sigma_{+}}{\sqrt{A_{+}}}, \frac{\partial^{\alpha} \sigma_{+}}{\sqrt{A_{+}}}-\frac{\partial^{\alpha} \sigma_{-}}{\sqrt{A_{-}}}\right\rangle \\
& -\frac{1}{\gamma \sqrt{A_{+}}}\left\langle\partial^{\alpha} \sigma_{+}, \partial^{\alpha} \phi\left(\sigma_{+}\right)-\partial^{\alpha} \phi\left(\sigma_{-}\right)\right\rangle+\frac{v_{+}}{\sqrt{\gamma} \sqrt{A_{+}}}\left\langle\nabla \partial^{\alpha} \sigma_{+}, \partial^{\alpha} v_{+}\right\rangle \\
& +\frac{\alpha_{+}}{\sqrt{\gamma} \sqrt{A_{+}}}\left\langle\nabla \partial^{\alpha} \sigma_{+}, \partial^{\alpha}\left(v_{+}-v_{-}\right)\right\rangle=-\frac{1}{\sqrt{A_{+}}}\left\langle\nabla \partial^{\alpha} \sigma_{+}, \partial^{\alpha} v_{+} \cdot \nabla v_{+}\right\rangle \\
& -\frac{1}{\sqrt{A_{+}}}\left\langle\nabla \partial^{\alpha} \sigma_{+}, v_{+} \cdot \nabla \partial^{\alpha} v_{+}\right\rangle-\frac{\gamma-1}{2 \sqrt{A_{+}}}\left\langle\nabla \partial^{\alpha} \sigma_{+}, \partial^{\alpha} \sigma_{+} \nabla \sigma_{-}\right\rangle \\
& -\frac{\gamma-1}{2 \sqrt{A_{+}}}\left\langle\nabla \partial^{\alpha} \sigma_{+}, \sigma_{+} \nabla \partial^{\alpha} \sigma_{+}\right\rangle+\frac{1}{\sqrt{A_{+}}}\left\langle\nabla \partial^{\alpha} \sigma_{+}, \partial^{\alpha} v_{+} \times \tilde{B}\right\rangle \\
& +\frac{1}{\sqrt{A_{+}}}\left\langle\nabla \partial^{\alpha} \sigma_{+}, v_{-} \times \partial^{\alpha} \tilde{B}\right\rangle+\frac{1}{\sqrt{A_{+}}}\left\langle\nabla \cdot \partial^{\alpha} v_{+}, \partial^{\alpha}\left(v_{+} \cdot \nabla \sigma_{+}\right)\right\rangle \\
& +\frac{\gamma-1}{2 \sqrt{A_{+}}}\left\langle\nabla \cdot \partial^{\alpha} v_{+}, \partial^{\alpha}\left(\sigma_{+} \nabla \cdot v_{+}\right)\right\rangle+\frac{1}{\sqrt{A_{+}}}\left\langle\nabla \cdot \partial^{\alpha} v_{+}, \nabla \cdot \partial^{\alpha} v_{+}\right\rangle .
\end{aligned}
$$


Now, applying $\partial^{\alpha}$ to (2.34), multipling by $\nabla \partial^{\alpha} \sigma_{-}$, and integrating in $x$ we get

$$
\begin{aligned}
& \left\langle\nabla \partial^{\alpha} \sigma_{-}, \partial_{t} \partial^{\alpha} v_{-}\right\rangle+\sqrt{A_{-}}\left\langle\nabla \partial^{\alpha} \sigma_{-}, \nabla \partial^{\alpha} \sigma_{-}\right\rangle-\frac{1}{\sqrt{\gamma}}\left\langle\nabla \partial^{\alpha} \sigma_{-}, \partial^{\alpha} \tilde{E}\right\rangle \\
& +\frac{v_{-}}{\sqrt{\gamma}}\left\langle\nabla \partial^{\alpha} \sigma_{-}, \partial^{\alpha} v_{-}\right\rangle+\frac{\alpha}{\sqrt{\gamma}}\left\langle\nabla \partial^{\alpha} \sigma_{-}, \partial^{\alpha}\left(v_{-}-v_{+}\right)\right\rangle=-\left\langle\nabla \partial^{\alpha} \sigma_{-}, \partial^{\alpha} v_{-} \cdot \nabla v_{-}\right\rangle \\
& -\left\langle\nabla \partial^{\alpha} \sigma_{-}, v_{-} \cdot \nabla \partial^{\alpha} v_{-}\right\rangle-\frac{\gamma-1}{2}\left\langle\nabla \partial^{\alpha} \sigma_{-}, \partial^{\alpha} \sigma_{-} \nabla \sigma_{-}\right\rangle-\frac{\gamma-1}{2}\left\langle\nabla \partial^{\alpha} \sigma_{-}, \sigma_{-} \nabla \partial^{\alpha} \sigma_{-}\right\rangle \\
& +\left\langle\nabla \partial^{\alpha} \sigma_{-}, \partial^{\alpha} v_{-} \times \tilde{B}\right\rangle+\left\langle\nabla \partial^{\alpha} \sigma_{-}, v_{-} \times \partial^{\alpha} \tilde{B}\right\rangle:=f_{2}^{-} .
\end{aligned}
$$

Since

$$
\left\langle\partial_{t} \partial^{\alpha} v_{-}, \nabla \partial^{\alpha} \sigma_{-}\right\rangle=\frac{\partial}{\partial_{t}}\left\langle\nabla \partial^{\alpha} \sigma_{-}, \partial^{\alpha} v_{-}\right\rangle-\left\langle\partial^{\alpha} v_{-}, \nabla \partial^{\alpha} \frac{\partial}{\partial_{t}} \sigma_{-}\right\rangle
$$

if we multiply (2.37) by $\frac{1}{\sqrt{A_{-}}}$then we obtain

$$
\begin{aligned}
\frac{\partial}{\partial_{t}} & \frac{1}{\sqrt{A_{-}}}\left\langle\nabla \partial^{\alpha} \sigma_{-}, \partial^{\alpha} v_{-}\right\rangle+\left\|\nabla \partial^{\alpha} \sigma_{-}\right\|^{2}-\frac{1}{\gamma}\left\langle\frac{\partial^{\alpha} \sigma_{-}}{\sqrt{A_{-}}}, \frac{\partial^{\alpha} \sigma_{+}}{\sqrt{A_{+}}}-\frac{\partial^{\alpha} \sigma_{-}}{\sqrt{A_{-}}}\right\rangle \\
& -\frac{1}{\gamma \sqrt{A_{-}}}\left\langle\partial^{\alpha} \sigma_{-}, \partial^{\alpha} \phi\left(\sigma_{+}\right)-\partial^{\alpha} \phi\left(\sigma_{-}\right)\right\rangle+\frac{v_{-}}{\sqrt{\gamma} \sqrt{A_{-}}}\left\langle\nabla \partial^{\alpha} \sigma_{-}, \partial^{\alpha} v_{-}\right\rangle \\
& +\frac{\alpha}{\sqrt{\gamma} \sqrt{A_{-}}}\left\langle\nabla \partial^{\alpha} \sigma_{-}, \partial^{\alpha}\left(v_{-}-v_{+}\right)\right\rangle=-\frac{1}{\sqrt{A_{-}}}\left\langle\nabla \partial^{\alpha} \sigma_{-}, \partial^{\alpha} v_{-} \cdot \nabla v_{-}\right\rangle \\
& -\frac{1}{\sqrt{A_{-}}}\left\langle\nabla \partial^{\alpha} \sigma_{-}, v_{-} \cdot \nabla \partial^{\alpha} v_{-}\right\rangle-\frac{\gamma-1}{2 \sqrt{A_{-}}}\left\langle\nabla \partial^{\alpha} \sigma_{-}, \partial^{\alpha} \sigma_{-} \nabla \sigma_{-}\right\rangle \\
& -\frac{\gamma-1}{2 \sqrt{A_{-}}}\left\langle\nabla \partial^{\alpha} \sigma_{-}, \sigma_{-} \nabla \partial^{\alpha} \sigma_{-}\right\rangle+\frac{1}{\sqrt{A_{+}}}\left\langle\nabla \partial^{\alpha} \sigma_{-}, \partial^{\alpha} v_{-} \times \tilde{B}\right\rangle \\
& +\frac{1}{\sqrt{A_{+}}}\left\langle\nabla \partial^{\alpha} \sigma_{-}, v_{-} \times \partial^{\alpha} \tilde{B}\right\rangle+\frac{1}{\sqrt{A_{-}}}\left\langle\nabla \cdot \partial^{\alpha} v_{-}, \partial^{\alpha}\left(v_{-} \cdot \nabla \sigma_{-}\right)\right\rangle \\
& +\frac{\gamma-1}{2 \sqrt{A_{-}}}\left\langle\nabla \cdot \partial^{\alpha} v_{-}, \partial^{\alpha}\left(\sigma_{-} \nabla \cdot v_{-}\right)\right\rangle+\frac{1}{\sqrt{A_{-}}}\left\langle\nabla \cdot \partial^{\alpha} v_{-}, \nabla \cdot \partial^{\alpha} v_{-}\right\rangle .
\end{aligned}
$$


Taking the sum of equatiosn (2.36) and (2.38) we obtain

$$
\begin{aligned}
\frac{\partial}{\partial_{t}}( & \left.\frac{1}{\sqrt{A_{+}}}\left\langle\nabla \partial^{\alpha} \sigma_{+}, \partial^{\alpha} v_{+}\right\rangle+\frac{1}{\sqrt{A_{-}}}\left\langle\nabla \partial^{\alpha} \sigma_{-}, \partial^{\alpha} v_{-}\right\rangle\right)+\left\|\nabla \partial^{\alpha}\left[\sigma_{-}, \sigma_{-}\right]\right\|^{2} \\
& +\frac{1}{\gamma}\left\langle\frac{\partial^{\alpha} \sigma_{+}}{\sqrt{A_{+}}}-\frac{\partial^{\alpha} \sigma_{-}}{\sqrt{A_{-}}}, \frac{\partial^{\alpha} \sigma_{+}}{\sqrt{A_{+}}}-\frac{\partial^{\alpha} \sigma_{-}}{\sqrt{A_{-}}}\right\rangle+\frac{1}{\gamma \sqrt{A_{-}}}\left\langle\partial^{\alpha} \sigma_{-}, \partial^{\alpha} \phi\left(\sigma_{+}\right)-\partial^{\alpha} \phi\left(\sigma_{-}\right)\right\rangle \\
& +\frac{1}{\gamma \sqrt{A_{+}}}\left\langle\partial^{\alpha} \sigma_{+}, \partial^{\alpha} \phi\left(\sigma_{+}\right)-\partial^{\alpha} \phi\left(\sigma_{-}\right)\right\rangle+\frac{v_{+}}{\sqrt{\gamma} \sqrt{A_{+}}}\left\langle\nabla \partial^{\alpha} \sigma_{+}, \partial^{\alpha} v_{+}\right\rangle \\
& +\frac{v_{-}}{\sqrt{\gamma} \sqrt{A_{-}}}\left\langle\nabla \partial^{\alpha} \sigma_{-}, \partial^{\alpha} v_{-}\right\rangle+\frac{\alpha}{\sqrt{\gamma} \sqrt{A_{+}}}\left\langle\nabla \partial^{\alpha} \sigma_{+}, \partial^{\alpha}\left(v_{+}-v_{-}\right)\right\rangle \\
& +\frac{\alpha-}{\sqrt{\gamma} \sqrt{A_{-}}}\left\langle\nabla \partial^{\alpha} \sigma_{-}, \partial^{\alpha}\left(v_{-}-v_{+}\right)\right\rangle=\frac{1}{\sqrt{A_{+}}}\left\|\nabla \cdot \partial^{\alpha} v_{+}\right\|^{2}+\frac{1}{\sqrt{A_{-}}}\left\|\nabla \cdot \partial^{\alpha} v_{-}\right\|^{2} \\
& +\left\langle\nabla \partial^{\alpha} \sigma_{+}, \partial^{\alpha} f_{2}^{+}\right\rangle+\left\langle\nabla \partial^{\alpha} \sigma_{-}, \partial^{\alpha} f_{2}^{-}\right\rangle-\left\langle\nabla \cdot \partial^{\alpha} v_{+}, \partial^{\alpha} f_{1}^{+}\right\rangle-\left\langle\nabla \partial^{\alpha} v_{-}, \partial^{\alpha} f_{1}^{-}\right\rangle .
\end{aligned}
$$

Applying the Cauchy-Schwarz inequality we obtain

$$
\begin{aligned}
& \frac{d}{d t}\left(\left\langle\partial^{\alpha} v_{+}, \nabla \partial^{\alpha} \sigma_{+}\right\rangle+\left\langle\partial^{\alpha} v_{-}, \nabla \partial^{\alpha} \sigma_{-}\right\rangle\right)+\lambda\left(\left\|\nabla\left[\sigma_{+}, \sigma_{-}\right]\right\|_{N-1}^{2}+\left\|\frac{\sigma_{+}}{\sqrt{A_{+}}}-\frac{\sigma_{-}}{\sqrt{A_{-}}}\right\|_{N}^{2}\right) \\
& \quad \leq C\left\|\left[v_{+}, v_{-}\right]\right\|_{N}^{2}+C\left\|\left[\sigma_{+}, \sigma_{-}\right]\right\|_{N-1}^{2} C\left\|\left[v_{+}, v_{-}\right]\right\|_{N}\|\nabla \tilde{B}\|_{N-2} \\
& \quad+C\left\|\left[\sigma_{+}, \sigma_{-}, v_{+}, v_{-}, \tilde{B}\right]\right\|_{N}^{2}\left\|\nabla\left[\sigma_{+}, \sigma_{-}, v_{+}, v_{-}\right]\right\|_{N-1}^{2} .
\end{aligned}
$$

Step (3) We want to show that

$$
\begin{aligned}
\frac{d}{d t} \varepsilon_{N, 2}^{i n t}(V)+\lambda\|\tilde{E}\|_{N-1}^{2} & \leq C\left\|\left[v_{+}, v_{-}\right]\right\|_{N}^{2}+C\left\|\left[\sigma_{+}, \sigma_{-}\right]\right\|_{N-1}^{2}+C\left\|\left[v_{+}, v_{-}\right]\right\|_{N}\|\nabla \tilde{B}\|_{N-2} \\
& +C\left\|\left[\sigma_{+}, \sigma_{-}, v_{+}, v_{-}, \tilde{B}\right]\right\|_{N}^{2}\left\|\nabla\left[\sigma_{+}, \sigma_{-}, v_{+}, v_{-}\right]\right\|_{N-1}^{2}
\end{aligned}
$$

with

$$
\varepsilon_{N, 2}^{i n t}(v)=\sum_{|\alpha| \leq N-1}\left\langle\partial^{\alpha}\left(v_{+}-v_{-}\right), \partial^{\alpha} \tilde{E}\right\rangle \text { for }|\alpha| \leq N-1 .
$$

To do so, we subtract equation (2.34) from equation (2.33) to get

$$
\begin{aligned}
\partial_{t}\left(v_{+}-v_{-}\right) & +\left(\sqrt{A_{+}} \nabla \sigma_{+}-\sqrt{A_{-}} \nabla \sigma_{-}\right)-\frac{2}{\sqrt{\gamma}} \tilde{E}=-\left(v_{+} \cdot \nabla v_{+}\right)+\left(v_{-} \cdot \nabla v_{-}\right) \\
& -\frac{\gamma-1}{2}\left(\sigma_{+} \nabla \sigma_{+}\right)+\frac{\gamma-1}{2}\left(\sigma_{-} \nabla \sigma_{-}\right)+\left(v_{+} \times \tilde{B}\right)+\left(v_{-} \times \tilde{B}\right) \\
& -\frac{1}{\sqrt{\gamma}}\left(v_{+} v_{+}-v_{-} v_{-}\right)-\frac{1}{\sqrt{\gamma}}\left[\alpha_{+}\left(v_{+}-v_{-}\right)-\alpha_{-}\left(v_{-}-v_{+}\right)\right]
\end{aligned}
$$


Now apply $\partial^{\alpha}$ to (2.40), multiply by $\partial^{\alpha} \tilde{E}$, integrate by parts in $x$, and replace $\partial^{\alpha} \tilde{E}$ with the third equation of (2.2). Then we get

$$
\begin{aligned}
& \left\langle\partial^{\alpha} \tilde{E}, \partial_{t} \partial^{\alpha}\left(v_{+}-v_{-}\right)\right\rangle-\frac{2}{\sqrt{\gamma}}\left\|\partial^{\alpha} \tilde{E}\right\|^{2}=-\left\langle\partial^{\alpha}\left(\sqrt{A_{+}} \nabla \sigma_{+}-\sqrt{A_{-}} \nabla \sigma_{-}\right), \partial^{\alpha} \tilde{E}\right\rangle \\
& -\left\langle\partial^{\alpha}\left(v_{+} \cdot \nabla v_{+}\right), \partial^{\alpha} \tilde{E}\right\rangle+\left\langle\partial^{\alpha}\left(v_{-} \cdot \nabla v_{-}\right), \partial^{\alpha} \tilde{E}\right\rangle-\frac{\gamma-1}{2}\left\langle\partial^{\alpha}\left(\sigma_{+} \nabla \sigma_{+}\right), \partial^{\alpha} \tilde{E}\right\rangle \\
& +\frac{\gamma-1}{2}\left\langle\partial^{\alpha}\left(\sigma_{-} \nabla \sigma_{-}\right), \partial^{\alpha} \tilde{E}\right\rangle+\left\langle\partial^{\alpha}\left(v_{+} \times \tilde{B}\right), \partial^{\alpha} \tilde{E}\right\rangle+\left\langle\partial^{\alpha}\left(v_{-} \times \tilde{B}\right), \partial^{\alpha} \tilde{E}\right\rangle \\
& -\frac{1}{\sqrt{\gamma}}\left\langle\partial^{\alpha}\left(v_{+} v_{+}-v_{-} v_{-}\right), \partial^{\alpha} \tilde{E}\right\rangle-\frac{1}{\sqrt{\gamma}}\left\langle\partial^{\alpha}\left[\alpha_{+}\left(v_{+}-v_{-}\right)-\alpha_{-}\left(v_{-}-v_{+}\right)\right], \partial^{\alpha} \tilde{E}\right\rangle .
\end{aligned}
$$

Since

$$
\left\langle\partial^{\alpha} \tilde{E}, \partial_{t} \partial^{\alpha}\left(v_{+}-v_{-}\right)\right\rangle=\frac{d}{d t}\left\langle\partial^{\alpha} \tilde{E}, \partial^{\alpha}\left(v_{+}-v_{-}\right)\right\rangle-\left\langle\partial^{\alpha} \partial_{t} \tilde{E}, \partial^{\alpha}\left(v_{+}-v_{-}\right)\right\rangle,
$$

we have

$$
\begin{aligned}
& \frac{d}{d t}\left\langle\partial^{\alpha} \tilde{E}, \partial^{\alpha}\left(v_{+}-v_{-}\right)\right\rangle-\frac{2}{\sqrt{\gamma}}\left\|\partial^{\alpha} \tilde{E}\right\|^{2}=\frac{1}{\sqrt{\gamma}}\left\|\partial^{\alpha}\left(v_{+}-v_{-}\right)\right\|^{2} \\
& \quad+\frac{1}{\sqrt{\gamma}}\left\langle\nabla \times \partial^{\alpha} \tilde{B}, \partial^{\alpha}\left(v_{+}-v_{-}\right)\right\rangle-\frac{1}{\sqrt{\gamma}}\left\langle\partial^{\alpha}\left[\phi\left(\sigma_{+}\right)+\frac{\sigma_{+}}{\sqrt{A_{+}}}\right] v_{+}, \partial^{\alpha}\left(v_{+}-v_{-}\right)\right\rangle \\
& \quad+\frac{1}{\sqrt{\gamma}}\left\langle\partial^{\alpha}\left[\phi\left(\sigma_{-}\right)+\frac{\sigma_{-}}{\sqrt{A_{-}}}\right] v_{-}, \partial^{\alpha}\left(v_{-}-v_{+}\right)\right\rangle-\frac{1}{\sqrt{\gamma}}\left\langle\partial^{\alpha}\left(v_{+} v_{+}-v_{-} v_{-}\right), \partial^{\alpha} \tilde{E}\right\rangle \\
& \quad-\frac{1}{\sqrt{\gamma}}\left\langle\partial^{\alpha}\left[\alpha_{+}\left(v_{+}-v_{-}\right)-\alpha_{-}\left(v_{-}-v_{+}\right)\right], \partial^{\alpha} \tilde{E}\right\rangle-\left\langle\partial^{\alpha}\left(\sqrt{A_{+}} \nabla \sigma_{+}-\sqrt{A_{-}} \nabla \sigma_{-}\right), \partial^{\alpha} \tilde{E}\right\rangle \\
& -\left\langle\partial^{\alpha}\left(f_{2}^{+}-f_{2}^{-}\right), \partial^{\alpha} \tilde{E}\right\rangle .
\end{aligned}
$$

Applying the Cauchy-Schwarz inequality we obtain

$$
\begin{gathered}
\frac{d}{d t}\left\langle\partial^{\alpha} \tilde{E}, \partial^{\alpha}\left(v_{+}-v_{-}\right)\right\rangle+\lambda\left\|\partial^{\alpha} \tilde{E}\right\|^{2} \leq C\left\|\partial^{\alpha}\left(v_{+}-v_{-}\right)\right\|^{2}+\left\|\left[v_{+}, v_{-}\right]\right\|^{2}\|\nabla \tilde{B}\|_{N-2} \\
\quad+C\left\|\nabla\left[\partial^{\alpha} \sigma_{+}-\partial^{\alpha} \sigma_{-}\right]\right\|^{2}+C\left\|\left[\sigma_{+}, \sigma_{-}, v_{+}, v_{-}, \tilde{B}\right]\right\|_{N}^{2}\left\|\nabla\left[\sigma_{+}, \sigma_{-}, v_{+}, v_{-}\right]\right\|_{N-1}^{2}
\end{gathered}
$$

Step (4) We want to show that

$$
\frac{d}{d t} \mathcal{E}_{N, 3}^{i n t}(V)+\lambda\|\nabla \tilde{B}\|_{N-2}^{2} \leq C\left\|\left[v_{+}, v_{-}, \tilde{E}\right]\right\|_{N-1}^{2}+C\left\|\left[\sigma_{+}, \sigma_{-}\right]\right\|_{N}^{2}\left\|\nabla\left[v_{+}, v_{-}\right]\right\|_{N-1}^{2},
$$

with

$$
\mathcal{E}_{N, 3}^{i n t}(v)=-\sum_{|\alpha| \leq N-2}\left\langle\nabla \times \partial^{\alpha} \tilde{E}, \partial^{\alpha} \tilde{B}\right\rangle \text { for all }|\alpha| \leq N-2
$$


Now apply $\partial^{\alpha}$ to the third equation of (2.2), multiply by $\nabla \times \partial^{\alpha} \tilde{B}$, and integrate by parts in $x$. We obtain

$$
\begin{aligned}
& \left\langle\partial_{t} \partial^{\alpha} \tilde{E}, \nabla \times \partial^{\alpha} \tilde{B}\right\rangle-\frac{1}{\sqrt{\gamma}}\left\|\nabla \times \partial^{\alpha} \tilde{B}\right\|^{2}=-\frac{1}{\sqrt{\gamma}}\left\langle\partial^{\alpha} v_{+}, \nabla \times \partial^{\alpha} \tilde{B}\right\rangle \\
& \quad+\frac{1}{\sqrt{\gamma}}\left\langle\partial^{\alpha} v_{-}, \nabla \times \partial^{\alpha} \tilde{B}\right\rangle-\frac{1}{\sqrt{\gamma}}\left\langle\partial^{\alpha}\left[\phi\left(\sigma_{+}\right)+\frac{\sigma_{+}}{\sqrt{A_{+}}}\right] v_{+}, \nabla \times \partial^{\alpha} \tilde{B}\right\rangle \\
& +\frac{1}{\sqrt{\gamma}}\left\langle\partial^{\alpha}\left[\phi\left(\sigma_{-}\right)+\frac{\sigma_{-}}{\sqrt{A_{-}}}\right] v_{-}, \nabla \times \partial^{\alpha} \tilde{B}\right\rangle .
\end{aligned}
$$

Since

$$
\left\langle\nabla \times \partial^{\alpha} \tilde{B}, \partial_{t} \partial^{\alpha} \tilde{E}\right\rangle=\frac{d}{d t}\left\langle\nabla \times \partial^{\alpha} \tilde{B}, \partial^{\alpha} \tilde{E}\right\rangle-\left\langle\nabla \times \partial^{\alpha} \partial_{t} \tilde{B}, \partial^{\alpha} \tilde{E}\right\rangle,
$$

we have

$$
\begin{aligned}
& \frac{d}{d t}\left\langle\nabla \times \partial^{\alpha} \tilde{B}, \partial^{\alpha} \tilde{E}\right\rangle-\left\langle\nabla \times \partial^{\alpha} \partial_{t} \tilde{B}, \partial^{\alpha} \tilde{E}\right\rangle-\frac{1}{\sqrt{\gamma}}\left\|\nabla \times \partial^{\alpha} \tilde{B}\right\|^{2}=-\frac{1}{\sqrt{\gamma}}\left\langle\partial^{\alpha} v_{+}, \nabla \times \partial^{\alpha} \tilde{B}\right\rangle \\
& \quad+\frac{1}{\sqrt{\gamma}}\left\langle\partial^{\alpha} v_{-}, \nabla \times \partial^{\alpha} \tilde{B}\right\rangle-\frac{1}{\sqrt{\gamma}}\left\langle\partial^{\alpha}\left[\phi\left(\sigma_{+}\right)+\frac{\sigma_{+}}{\sqrt{A_{+}}}\right] v_{+}, \nabla \times \partial^{\alpha}\right\rangle \\
& \quad+\frac{1}{\sqrt{\gamma}}\left\langle\partial^{\alpha}\left[\phi\left(\sigma_{-}\right)+\frac{\sigma_{-}}{\sqrt{A_{-}}}\right] v_{-}, \nabla \times \partial^{\alpha} \tilde{B}\right\rangle .
\end{aligned}
$$

From the fourth equation of (2.2) substituting $\nabla \times \tilde{E}$ for $\partial_{t} \tilde{B}$ we get

$$
\begin{aligned}
\frac{d}{d t} & \left\langle\nabla \times \partial^{\alpha} \tilde{B}, \partial^{\alpha} \tilde{E}\right\rangle-\frac{1}{\sqrt{\gamma}}\left\langle\nabla \times \partial^{\alpha}(\nabla \times \tilde{E}), \partial^{\alpha} \tilde{E}\right\rangle-\frac{1}{\sqrt{\gamma}}\left\|\nabla \times \partial^{\alpha} \tilde{B}\right\|^{2} \\
& =-\frac{1}{\sqrt{\gamma}}\left\langle\partial^{\alpha} v_{+}, \nabla \times \partial^{\alpha} \tilde{B}\right\rangle+\frac{1}{\sqrt{\gamma}}\left\langle\partial^{\alpha} v_{-}, \nabla \times \partial^{\alpha} \tilde{B}\right\rangle \\
& -\frac{1}{\sqrt{\gamma}}\left\langle\partial^{\alpha}\left[\phi\left(\sigma_{+}\right)+\frac{\sigma_{+}}{\sqrt{A_{+}}}\right] v_{+}, \nabla \times \partial^{\alpha} \tilde{B}\right\rangle \\
& +\frac{1}{\sqrt{\gamma}}\left\langle\partial^{\alpha}\left[\phi\left(\sigma_{-}\right)+\frac{\sigma_{-}}{\sqrt{A_{-}}}\right] v_{-}, \nabla \times \partial^{\alpha} \tilde{B}\right\rangle .
\end{aligned}
$$

Multiplying this result by -1 we obtain

$$
\begin{aligned}
& -\frac{d}{d t}\left\langle\nabla \times \partial^{\alpha} \tilde{B}, \partial^{\alpha} \tilde{E}\right\rangle+\frac{1}{\sqrt{\gamma}}\left\|\nabla \times \partial^{\alpha} \tilde{B}\right\|^{2}=\frac{1}{\sqrt{\gamma}}\left\|\nabla \times \partial^{\alpha} \tilde{E}\right\|^{2} \\
& +\frac{1}{\sqrt{\gamma}}\left\langle\partial^{\alpha} v_{+}, \nabla \times \partial^{\alpha} \tilde{B}\right\rangle-\frac{1}{\sqrt{\gamma}}\left\langle\partial^{\alpha} v_{-}, \nabla \times \partial^{\alpha} \tilde{B}\right\rangle \\
& \quad+\frac{1}{\sqrt{\gamma}}\left\langle\partial^{\alpha}\left[\phi\left(\sigma_{+}\right)+\frac{\sigma_{+}}{\sqrt{A_{+}}}\right] v_{+}, \nabla \times \partial^{\alpha} \tilde{B}\right\rangle \\
& -\frac{1}{\sqrt{\gamma}}\left\langle\partial^{\alpha}\left[\phi\left(\sigma_{-}\right)+\frac{\sigma_{-}}{\sqrt{A_{-}}}\right] v_{-}, \nabla \times \partial^{\alpha} \tilde{B}\right\rangle .
\end{aligned}
$$


Applying the Cauchy-Schwarz inequality and summing over $|\alpha| \leq N-2$, also we use the relation

$$
\left\|\partial^{\alpha} \partial_{x i} \tilde{B}\right\|=\left\|\partial_{x i} \Delta^{-1} \nabla \times\left(\nabla \times \partial^{\alpha} \tilde{B}\right)\right\| \leq\left\|\nabla \times \partial^{\alpha} \tilde{B}\right\|
$$

for each $1 \leq i \leq 3$. Then we obtain

$$
\begin{aligned}
& -\frac{d}{d t}\left\langle\nabla \times \partial^{\alpha} \tilde{B}, \partial^{\alpha} \tilde{E}\right\rangle+\lambda\left\|\nabla \times \partial^{\alpha} \tilde{B}\right\|_{N-2}^{2} \leq C\left\|\left[v_{+}, v_{-}, \tilde{E}\right]\right\|_{N-1}^{2} \\
& +C\left\|\left[\sigma_{+}, \sigma_{-}\right]\right\|_{N}^{2}\left\|\nabla\left[v_{+}, v_{-}\right]\right\|_{N-1}^{2} .
\end{aligned}
$$

Step(5) Utilizing steps (1)-(4) above we can now prove (2.18). Define

$$
\varepsilon_{N}(V(t))=\|\nabla V(t)\|_{N}^{2}+\sum_{i=1}^{3} \varepsilon_{N}^{i n t}(V(t)),
$$

and note that constants $0<k_{3} \ll k_{2} \ll k_{1} \ll 1$ are to be determined. We observe that if $0<k_{i} \ll 1$ for $i=1,2,3$, are sufficiently small then $\mathcal{E}_{N}(V) \sim\|V\|_{N}^{2}$ holds. Furthermore, by letting $0 \ll k_{3} \ll k_{2} \ll k_{1} \ll 1$ be sufficiently small, taking $k_{2}^{3 / 2} \ll k_{3}$, and taking the sum of (2.19), $k_{1} \times(2.30), k_{2} \times(2.39)$, and $k_{1} \times(2.41)$ we find there exists $\lambda>0, C>0$ such that (2.18) is satisfied:

$$
\begin{aligned}
& \frac{1}{2} \frac{d}{d t}\|V\|_{N}^{2}+\frac{v_{ \pm}}{\sqrt{\gamma}}\left\|\left[v_{+}, v_{-}\right]\right\|_{N}^{2}+k_{1} \frac{d}{d t} \sum_{|\alpha| \leq N}\left(\left\langle\partial^{\alpha} v_{+}, \nabla \partial^{\alpha} \sigma_{+}\right\rangle+\left\langle\partial^{\alpha} v_{-}, \nabla \partial^{\alpha} \sigma_{-}\right\rangle\right) \\
& \quad+k_{1} \lambda\left(\left\|\nabla\left[\sigma_{+}, \sigma_{-}\right]\right\|_{N-1}^{2}+\left\|\frac{\sigma_{+}}{\sqrt{A_{+}}}-\frac{\sigma_{-}}{\sqrt{A_{-}}}\right\|_{N}^{2}\right) \\
& \quad+k_{2} \frac{d}{d t} \sum_{|\alpha| \leq N-1}\left\langle\partial^{\alpha}\left(v_{+}-v_{-}\right), \partial^{\alpha} \tilde{E}\right\rangle \\
& \quad+k_{2} \lambda\|\tilde{E}\|_{N-1}^{2}-k_{3} \frac{d}{d t} \sum_{|\alpha| \leq N-2}\left\langle\nabla \times \partial^{\alpha} \tilde{E}, \partial^{\alpha} \tilde{B}\right\rangle+k_{3} \lambda\|\nabla \tilde{B}\|_{N-2}^{2} \\
& \quad \leq C\|V\|_{N}\left(\left\|\left[v_{+}, v_{-}\right]\right\|^{2}+\left\|\nabla\left[\sigma_{+}, \sigma_{-}, v_{+}, v_{-}\right]\right\|_{N-1}^{2}\right)+k_{1} C\left\|\left[v_{+}, v_{-}\right]\right\|_{N}^{2} \\
& \quad+k_{1} C\left\|\left[\sigma_{+}, \sigma_{-}, v_{+}, v_{-}, \tilde{B}\right]\right\|_{N}^{2}\left\|\nabla\left[\sigma_{+}, \sigma_{-}, v_{+}, v_{-}\right]\right\|_{N-1}^{2} \\
& \quad+k_{2} C\left\|\left[v_{+}, v_{-}\right]\right\|_{N}^{2}+k_{2} C\left\|\left[\sigma_{+}, \sigma_{-}\right]\right\|_{N-1}^{2}+k_{2} C\left\|\left[v_{+}, v_{-}\right]\right\|\left\|_{N}\right\| \nabla \tilde{B} \|_{N-2} \\
& \quad+k_{2} C\left\|\left[\sigma_{+}, \sigma_{-}, v_{+}, v_{-}, \tilde{B}\right]\right\|_{N}^{2}\left\|\nabla\left[\sigma_{+}, \sigma_{-}, v_{+}, v_{-}\right]\right\|_{N-1}^{2} \\
& \quad+k_{3} C\left\|\left[v_{+}, v_{-}, \tilde{E}\right]\right\|_{N-1}^{2}+k_{3} C\left\|\left[\sigma_{+}, \sigma_{-}\right]\right\|_{N}^{2}\left\|\nabla\left[v_{+}, v_{-}\right]\right\|_{N-1}^{2} \cdot
\end{aligned}
$$

If we now let

$$
\|V\| \leq \mathcal{E}^{\frac{1}{2}}, \quad\left\|\left[\sigma_{+}, \sigma_{-}, v_{+}, v_{-}, \tilde{B}\right]\right\|_{N}^{2}=\mathcal{E}_{N},\left\|\nabla\left[\sigma_{+}, \sigma_{-}, v_{+}, v_{-}\right]\right\|_{N-1}^{2}=\mathcal{D}_{N},
$$


then

$$
\begin{aligned}
& \frac{d}{d t}\left[\|V\|_{N}^{2}+k_{1} \sum_{|\alpha| \leq N}\left(\left\langle\partial^{\alpha} v_{+}, \nabla \partial^{\alpha} \sigma_{+}\right\rangle+\left\langle\partial^{\alpha} v_{-}, \nabla \partial^{\alpha} \sigma_{-}\right\rangle\right)\right. \\
& \left.\quad+k_{2} \sum_{|\alpha| \leq N-1}\left\langle\partial^{\alpha}\left(v_{+}-v_{-}\right), \partial^{\alpha} E\right\rangle-k_{3} \frac{d}{d t} \sum_{|\alpha| \leq N-2}\left\langle\nabla \times \partial^{\alpha} \tilde{E}, \partial^{\alpha} \tilde{B}\right\rangle\right] \\
& \quad+\frac{v_{ \pm}}{\sqrt{\gamma}}\left\|\left[v_{+}, v_{-}\right]\right\|_{N}^{2}+k_{2} \lambda\left\|\nabla\left[\sigma_{+}, \sigma_{-}\right]\right\|_{N-1}^{2}+k_{3} \lambda\|\nabla \tilde{B}\|_{N-2}^{2}+k_{2} \lambda\|\nabla \tilde{E}\|_{N-2}^{2} \\
& \quad+k_{1} \lambda\left\|\frac{\sigma_{+}}{\sqrt{A_{+}}}-\frac{\sigma_{-}}{\sqrt{A_{-}}}\right\|_{N}^{2} \leq C\left[\|V\|_{N}\left\|\nabla\left[\sigma_{+}, \sigma_{-}, v_{+}, v_{-}\right]\right\|_{N-1}^{2}\right. \\
& \left.\quad+k_{1} C\left\|\left[\sigma_{+}, \sigma_{-}, v_{+}, v_{-}, \tilde{B}\right]\right\|_{N}^{2}\left\|\nabla\left[\sigma_{+}, \sigma_{-}, v_{+}, v_{-}\right]\right\|_{N-1}^{2}\right] .
\end{aligned}
$$

It follows that

$$
\begin{aligned}
& \frac{d}{d t} \varepsilon_{N}(V(t))+\frac{v_{ \pm}}{\sqrt{\gamma}}\left\|\left[v_{+}, v_{-}\right]\right\|_{N}^{2}+k_{2} \lambda\left\|\nabla\left[\sigma_{+}, \sigma_{-}\right]\right\|_{N-1}^{2}+k_{3} \lambda\|\nabla \tilde{B}\|_{N-2}^{2} \\
& \quad+k_{2} \lambda\|\nabla \tilde{E}\|_{N-2}^{2}+k_{2} \lambda\|\tilde{E}\|^{2}+k_{1} \lambda\left\|\frac{\sigma_{+}}{\sqrt{A_{+}}}-\frac{\sigma_{-}}{\sqrt{A_{-}}}\right\|_{N}^{2} \leq C\left[\left(\|V\|_{N}\right.\right. \\
& \left.\left.\quad+\left\|\nabla\left[\sigma_{+}, \sigma_{-}, v_{+}, v_{-}\right]\right\|_{N-1}^{2}\right)\left\|\nabla\left[\sigma_{+}, \sigma_{-}, v_{+}, v_{-}\right]\right\|_{N-1}^{2}\right] .
\end{aligned}
$$

Thus

$$
\frac{d}{d t} \mathcal{E}_{N}(V(t))+\lambda \mathcal{D}_{N}(V(t)) \leq C\left[\mathcal{E}_{N}(V(t))^{1 / 2}+\mathcal{E}_{N}(V(t))\right] \mathcal{D}_{N}(V(t)),
$$

and this concludes the proof.

\subsection{Proof of global existence}

We consider the global existence of the smooth solution to the isentropic EulerMaxwell system for quasi-linear symmetric hyperbolic system (2.2). Therefore, we combine those a priori estimates with the local existence of solutions to extend the local solution up to infinite time by using of the continuity of $\varepsilon_{N}(V(t))$.

Lemma 2.2. (Local existence of smooth solution [1],[5],[6]). Asume $V_{0} \in H^{N}\left(\mathbb{R}^{3}\right)$ satisfies (2.4). Then there exists $T_{0}>0$ such that the Cauchy problem (2.2), (2.3) admits a unique solution on $\left[0, T_{0}\right)$ with

$$
V \in C\left(\left[0, T_{0}\right) ; H^{N}\left(\mathbb{R}^{3}\right)\right) \cap \operatorname{Lip}\left(\left[0, T_{0}\right) ; H^{N-1}\left(\mathbb{R}^{3}\right)\right) .
$$




\section{Proof of Proposition 2.1}

Since (2.2) is a quasi-linear symmetric hyperbolic system, the global existence of smooth solutions follows from the local existence result in Lemma 2.2, (see also Section 16 of [6]). In addition, the a priori estimate (2.18) in Theorem 2.1 and the continuity argument show that $\varepsilon_{N}(V(t))$ is bounded uniformly in time under the assumption that $\varepsilon_{N}\left(V_{0}\right)>0$ is sufficiently small. Therefore, global solutions satisfying (2.14) and (2.15) exist. This concludes the proof of Proposition 2.1. 


\section{Chapter 3}

\section{Linearized Homogeneous System}

\subsection{Linearized equations}

To obtain the time-decay rates of solution to the nonlinear system (2.2) or (2.5), we consider the linearized homogeneous equations of system (2.5).

$$
\left\{\begin{array}{l}
\partial_{t} \rho_{ \pm}+\nabla \cdot u_{ \pm}=0, \\
\partial_{t} u_{ \pm}+u_{ \pm}+\alpha_{ \pm} u_{ \pm}-\alpha_{ \pm} u_{\mp} \mp E+A_{ \pm} \gamma \nabla \rho_{ \pm}=0, \\
\partial_{t} E-\nabla \times B-u_{-}+u_{+}=0, \\
\partial_{t} B+\nabla \times E=0, \\
\nabla . E=\rho_{+}-\rho_{-}, \quad \nabla . B=0 . \quad t>0, \quad x \in \mathbb{R}^{3},
\end{array}\right.
$$

with given initial data

$$
\left.U\right|_{t=0}=U_{0}:=\left[\rho_{ \pm 0}, u_{ \pm 0}, E_{0}, B_{0}\right], \quad x \in \mathbb{R}^{3}
$$

which satisfies the compatibility conditions

$$
\nabla \cdot E_{0}=\rho_{+0}-\rho_{-0}, \quad \nabla \cdot B_{0}=0, \quad x \in \mathbb{R}^{3} .
$$

Throughout this section, we let $U=\left[\rho_{ \pm}, u_{ \pm}, E, B\right]$ be the solution to system (3.1). Moreover, in this section, we introduce some notation about Fourier transform $f$ : $\mathbb{R}^{3} \rightarrow \mathbb{R}$, defined by

$$
\hat{f}(k)=\int_{\mathbb{R}^{3}} e^{-i x \cdot k} f(x) d x, \quad x \cdot k:=\sum_{j=1}^{3} x_{j} k_{j}, k \in \mathbb{R}^{3},
$$

where $i$ is complex number, and we use the energy mothd to the initial problem (3.1), (3.3) in Fourier space to show that there is a time-frequency Lyapunov inequality, 
which leads to the pointwise time-frequency upper-bound of the solution.

We will use the energy method to the initial value problem (3.1), (3.3) in the Fourier transform to show that there is a time-frequency Lyapunov functional which is equivalent with $|\hat{U}(t, k)|^{2}$ and morethen its dissipation rate can be represented by itself. The main result of this section state as follows.

Theorem 3.1. Let $U, t>0, x \in R^{3}$, be a well-defined solution to the system (3.1). There is a time-frequency Lyapunov functional $\mathcal{E}(\hat{U}(t, k))$ with

$$
\mathcal{E}(\hat{U}) \sim|\hat{U}|^{2}:=\left|\left[\hat{\rho}_{+}, \hat{\rho}_{-}\right]\right|^{2}+\left|\hat{\rho}_{+}-\hat{\rho}_{-}\right|^{2}+\left|\left[\hat{u}_{+}, \hat{u}_{-}\right]\right|^{2}+|\hat{E}|^{2}+|\hat{B}|^{2}
$$

satisfying that there is $\lambda>0$ such that the Lyapunove inequality

$$
\frac{d}{d t} \mathcal{E}(\hat{U}(t, k))+\lambda\left\|\left[\hat{\rho}_{+}, \hat{\rho}_{-},\left(\hat{\rho}_{+}-\hat{\rho}_{-}\right), \hat{u}_{+}, \hat{u}_{-}\right]\right\|^{2}+\frac{\lambda|k|^{2}\|[\hat{E}, \hat{B}]\|^{2}}{\left(1+|k|^{2}\right)^{2}} \leq 0
$$

holds for any $t>0$ and $k \in \mathbb{R}^{3}$

Proof : We consider the linearized homogeneous system (3.1) in Fourier space. For this purpose by taking Fourier transform in $x$ for linearized homogeneous system (3.1), $\hat{U}=\left[\hat{\rho}_{ \pm}, \hat{u}_{ \pm}, \hat{E}, \hat{B}\right]$ satisfies.

$$
\left\{\begin{array}{l}
\partial_{t} \hat{\rho}_{ \pm}+i k \cdot \hat{u}_{ \pm}=0, \\
\partial_{t} \hat{u}_{ \pm}+\hat{u}_{ \pm}+\alpha_{ \pm} \hat{u}_{ \pm}-\alpha_{ \pm} \hat{u}_{\mp} \mp \hat{E}+A_{ \pm} \gamma i k \hat{\rho_{ \pm}}=0, \\
\partial_{t} \hat{E}-i k \times \hat{B}-\hat{u}_{-}+\hat{u}_{+}=0, \\
\partial_{t} \hat{B}+i k \times \hat{E}=0, \\
i k \cdot \hat{E}=\hat{\rho}_{+}-\hat{\rho}_{-}, \quad i k \cdot \hat{B}=0 . \quad t>0, \quad x \in \mathbb{R}^{3} .
\end{array}\right.
$$

First, multiply the first equation by $\hat{\rho}_{ \pm}$and $A_{ \pm} \gamma$, the second equation by $\hat{u}_{ \pm}$, the third equation by $\hat{E}$ and the fourth equation by $\hat{B}$ and taking the summation of all equations.

$$
\begin{aligned}
& A_{+} \gamma \partial_{t}\left\langle\hat{\rho}_{+}, \hat{\rho}_{+}\right\rangle+A_{+} \gamma\left\langle i k \cdot \hat{u}_{+}, \hat{\rho}_{+}\right\rangle=0 \\
& A_{-} \gamma \partial_{t}\left\langle\hat{\rho}_{-}, \hat{\rho}_{-}\right\rangle+A_{-} \gamma\left\langle i k \cdot \hat{u}_{-}, \hat{\rho}_{-}\right\rangle=0 \\
& \partial_{t}\left\langle\hat{u}_{+}, \hat{u}_{+}\right\rangle+\left\langle\hat{u}_{+}, \hat{u}_{+}\right\rangle+\alpha_{+}\left\langle\hat{u}_{+}, \hat{u}_{+}\right\rangle-\alpha_{+}\left\langle\hat{u}_{-}, \hat{u}_{+}\right\rangle-\left\langle\hat{E}, \hat{u}_{+}\right\rangle+A_{+} \gamma\left\langle i k \hat{\rho}_{+}, \hat{u}_{+}\right\rangle=0 \\
& \partial_{t}\left\langle\hat{u}_{-}, \hat{u}_{-}\right\rangle+\left\langle\hat{u}_{-}, \hat{u}_{-}\right\rangle+\alpha_{-}\left\langle\hat{u}_{-}, \hat{u}_{-}\right\rangle-\alpha_{-}\left\langle\hat{u}_{+}, \hat{u}_{-}\right\rangle+\left\langle\hat{E}, \hat{u}_{-}\right\rangle+A_{-} \gamma\left\langle i k \hat{\rho}_{-}, \hat{u}_{-}\right\rangle=0 \\
& \partial_{t}\langle\hat{E}, \hat{E}\rangle-\langle i k \times \hat{B}, \hat{E}\rangle-\left\langle\hat{u}_{+}, \hat{E}\right\rangle+\left\langle\hat{u}_{-}, \hat{E}\right\rangle=0 \\
& \partial_{t}\langle\hat{B}, \hat{B}\rangle+\langle i k \times \hat{E}, \hat{B}\rangle=0 .
\end{aligned}
$$

Thus,

$$
\begin{aligned}
& \frac{1}{2} \frac{d}{d t}\left\|\left[\sqrt{A_{+} \gamma} \hat{\rho}_{+}, \sqrt{A_{-} \gamma} \hat{\rho}_{-}, \hat{u}_{+}, \hat{u}_{-}, \hat{E}, \hat{B}\right]\right\|^{2}+\left\|\left[\hat{u}_{+}, \hat{u}_{-}\right]\right\|^{2}+\left\|\left[\alpha_{+} \hat{u}_{+}, \alpha_{-} \hat{u}_{-}\right]\right\|^{2} \\
& =\alpha_{+}\left\langle\hat{u}_{-}, \hat{u}_{+}\right\rangle+\alpha_{-}\left\langle\hat{u}_{+}, \hat{u}_{-}\right\rangle .
\end{aligned}
$$


By Cauchy-Schwarz inequality implies

$$
\frac{1}{2} \frac{d}{d t}\left\|\left[\sqrt{A_{+} \gamma} \hat{\rho}_{+}, \sqrt{A_{-} \gamma} \hat{\rho}_{-}, \hat{u}_{+}, \hat{u}_{-}, \hat{E}, \hat{B}\right]\right\|^{2}+C\left\|\left[\hat{u}_{+}, \hat{u}_{-}\right]\right\|^{2} \leq 0 .
$$

Then, we multiply the second equation of (3.6) by $i k \hat{\rho}_{ \pm}$using integration by parts in $t$ and replacing $\partial_{t} \hat{\rho}_{ \pm}$by the first equation of (3.6).

$\left\langle\partial_{t} \cdot \hat{u}_{ \pm}, i k \hat{\rho}_{ \pm}\right\rangle+\left\langle\hat{u}_{ \pm}, i k \hat{\rho}_{ \pm}\right\rangle+\alpha_{ \pm}\left\langle\hat{u}_{ \pm}, i k \hat{\rho}_{ \pm}\right\rangle-\alpha_{ \pm}\left\langle\hat{u}_{\mp}, i k \hat{\rho}_{ \pm}\right\rangle \mp\left\langle\hat{E}, i k \hat{\rho}_{ \pm}\right\rangle+A_{ \pm} \gamma\left\langle i k \hat{\rho}_{ \pm}, i k \hat{\rho}_{ \pm}\right\rangle$,

since

$$
\left\langle\partial_{t} \cdot \hat{u}_{ \pm}, i k \hat{\rho}_{ \pm}\right\rangle=\frac{d}{d t}\left\langle\hat{u}_{ \pm}, i k \hat{\rho}_{ \pm}\right\rangle-\left\langle\partial_{t} i k \hat{\rho}_{ \pm}, \hat{u}_{ \pm}\right\rangle
$$

we get

$$
\begin{aligned}
& \frac{d}{d t}\left\langle\hat{u}_{ \pm}, i k \hat{\rho}_{ \pm}\right\rangle-\left\langle\partial_{t} i k \hat{\rho}_{ \pm}, \hat{u}_{ \pm}\right\rangle+\left\langle\hat{u}_{ \pm}, i k \hat{\rho}_{ \pm}\right\rangle+\alpha_{ \pm}\left\langle\hat{u}_{ \pm}, i k \hat{\rho}_{ \pm}\right\rangle-\alpha_{ \pm}\left\langle\hat{u}_{\mp}, i k \hat{\rho}_{ \pm}\right\rangle \\
& \mp\left\langle\hat{E}, i k \hat{\rho}_{ \pm}\right\rangle+A_{ \pm} \gamma\left\langle i k \hat{\rho}_{ \pm}, i k \hat{\rho}_{ \pm}\right\rangle .
\end{aligned}
$$

Now, we split the solution to tow equations.

$$
\begin{aligned}
& \frac{d}{d t}\left\langle\hat{u}_{+}, i k \hat{\rho}_{+}\right\rangle-\left\langle\partial_{t} i k \hat{\rho}_{+}, \hat{u}_{+}\right\rangle+\left\langle\hat{u}_{+}, i k \hat{\rho}_{+}\right\rangle+\alpha_{+}\left\langle\hat{u}_{+}, i k \hat{\rho}_{+}\right\rangle-\alpha_{+}\left\langle\hat{u}_{-}, i k \hat{\rho}_{+}\right\rangle \\
& -\left\langle\hat{E}, i k \hat{\rho}_{+}\right\rangle+A_{+} \gamma\left\langle i k \hat{\rho}_{+}, i k \hat{\rho}_{+}\right\rangle
\end{aligned}
$$

and

$$
\begin{aligned}
& \frac{d}{d t}\left\langle\hat{u}_{-}, i k \hat{\rho}_{-}\right\rangle-\left\langle\partial_{t} i k \hat{\rho}_{-}, \hat{u}_{-}\right\rangle+\left\langle\hat{u}_{-}, i k \hat{\rho}_{-}\right\rangle+\alpha_{-}\left\langle\hat{u}_{-}, i k \hat{\rho}_{-}\right\rangle-\alpha_{-}\left\langle\hat{u}_{+}, i k \hat{\rho}_{-}\right\rangle \\
& +\left\langle\hat{E}, i k \hat{\rho}_{-}\right\rangle-A_{-} \gamma\left\langle i k \hat{\rho}_{-}, i k \hat{\rho}_{-}\right\rangle .
\end{aligned}
$$

Taking the sum of equations (3.9) and (3.10)

$$
\begin{aligned}
& \frac{d}{d t}\left\langle\hat{u}_{+}, i k \hat{\rho}_{+}\right\rangle+\frac{d}{d t}\left\langle\hat{u}_{-}, i k \hat{\rho}_{-}\right\rangle-|k|^{2}\left\|\left[\hat{u}_{+}, \hat{u}_{-}\right]\right\|^{2}+\left\langle\hat{u}_{+}, i k \hat{\rho}_{+}\right\rangle+\left\langle\hat{u}_{-}, i k \hat{\rho}_{-}\right\rangle \\
& -\left\langle\hat{u}_{-}, i k\left(\alpha_{+} \hat{\rho}_{+}-\alpha_{-} \hat{\rho}_{-}\right)\right\rangle+\left\langle\hat{u}_{+}, i k\left(\alpha_{+} \hat{\rho}_{+}-\alpha_{-} \hat{\rho}_{-}\right)\right\rangle+\left\langle\hat{\rho}_{-}-\hat{\rho}_{+}, \hat{\rho}_{-}-\hat{\rho}_{+}\right\rangle \\
& +A_{+} \gamma|k|^{2}\left\|\hat{\rho}_{+}\right\|^{2}+A_{-} \gamma|k|^{2}\left\|\hat{\rho}_{-}\right\|^{2}=0 .
\end{aligned}
$$

Thus, we obtain

$$
\begin{aligned}
& \frac{d}{d t}\left\langle\hat{u}_{+}, i k \hat{\rho}_{+}\right\rangle+\frac{d}{d t}\left\langle\hat{u}_{-}, i k \hat{\rho}_{-}\right\rangle+A_{+} \gamma|k|^{2}\left\|\hat{\rho}_{+}\right\|^{2}+A_{-} \gamma|k|^{2}\left\|\hat{\rho}_{-}\right\|^{2}+\left\|\hat{\rho}_{-}-\hat{\rho}_{+}\right\|^{2} \\
& =|k|^{2}\left\|\left[\hat{u}_{+}, \hat{u}_{-}\right]\right\|^{2}-\left\langle\hat{u}_{+}, i k \hat{\rho}_{+}\right\rangle-\left\langle\hat{u}_{-}, i k \hat{\rho}_{-}\right\rangle+\left\langle\hat{u}_{-}, i k\left(\alpha_{+} \hat{\rho}_{+}-\alpha_{-} \hat{\rho}_{-}\right)\right\rangle \\
& -\left\langle\hat{u}_{+}, i k\left(\alpha_{+} \hat{\rho}_{+}-\alpha_{-} \hat{\rho}_{-}\right)\right\rangle .
\end{aligned}
$$


Moreover, taking the real part and using the Cauchy-Schwarz inequality implies

$$
\begin{aligned}
& \frac{d}{d t} R\left\langle\hat{u}_{+}, i k \hat{\rho}_{+}\right\rangle+\frac{d}{d t} R\left\langle\hat{u}_{-}, i k \hat{\rho}_{-}\right\rangle+\lambda|k|^{2}\left\|\hat{\rho}_{+}\right\|^{2}+\lambda|k|^{2}\left\|\hat{\rho}_{-}\right\|^{2}+\lambda\left\|\hat{\rho}_{-}-\hat{\rho}_{+}\right\|^{2} \\
& \leq C|k|^{2}\left\|\left[\hat{u}_{+}, \hat{u}_{-}\right]\right\|^{2} .
\end{aligned}
$$

Now, dividing by $\left(1+|k|^{2}\right)$

$$
\begin{gathered}
\frac{d}{d t} \frac{R\left\langle\hat{u}_{+}, i k \hat{\rho}_{+}\right\rangle}{1+|k|^{2}}+\frac{d}{d t} \frac{R\left\langle\hat{u}_{-}, i k \hat{\rho}_{-}\right\rangle}{1+|k|^{2}}+\frac{\lambda|k|^{2}\left(\left\|\hat{\rho}_{+}\right\|^{2}+\left\|\hat{\rho}_{-}\right\|^{2}\right)}{1+|k|^{2}} \\
+\frac{\lambda|k|^{2}\left\|\hat{\rho}_{-}\right\|^{2}}{1+|k|^{2}}+\frac{\lambda\left\|\hat{\rho}_{-}-\hat{\rho}_{+}\right\|^{2}}{1+|k|^{2}} \leq \frac{C|k|^{2}\left\|\left[\hat{u}_{+}, \hat{u}_{-}\right]\right\|^{2}}{1+|k|^{2}}
\end{gathered}
$$

Similarly, taking the second equation of (3.6) and multiply by $\hat{E}$, then using integration by parts in $t$.

$$
\left\langle\partial_{t} \hat{u}_{ \pm}, \hat{E}\right\rangle+\left\langle\hat{u}_{ \pm}, \hat{E}\right\rangle+\alpha_{ \pm}\left\langle\hat{u}_{ \pm}, \hat{E}\right\rangle-\alpha_{ \pm}\left\langle\hat{u}_{ \pm}, \hat{E}\right\rangle \mp\langle\hat{E}, \hat{E}\rangle+\left\langle A_{ \pm} \gamma i k \hat{\rho}_{ \pm}, \hat{E}\right\rangle=0
$$

Since

$$
\left\langle\partial_{t} \hat{u}_{ \pm}, \hat{E}\right\rangle=\frac{d}{d t}\left\langle\hat{u}_{ \pm}, \hat{E}\right\rangle-\left\langle\partial_{t} \hat{E}, \hat{u}_{ \pm}\right\rangle
$$

Thus,

$$
\left\langle\partial_{t} \hat{E}, \hat{u}_{ \pm}\right\rangle+\left\langle\hat{E}, \hat{u}_{ \pm}\right\rangle+\alpha_{ \pm}\left\langle\hat{u}_{ \pm}, \hat{E}\right\rangle-\alpha_{ \pm}\left\langle\hat{u}_{ \pm}, \hat{E}\right\rangle \mp\|\hat{E}\|^{2}+A_{ \pm} \gamma\left\langle i k \hat{\rho}_{ \pm}, \hat{E}\right\rangle=0 .
$$

Now, using third equation of (3.6) and replacing $\partial_{t} \hat{E}$.

$$
\begin{aligned}
& \frac{d}{d t}\left\langle\hat{u}_{+}, \hat{E}\right\rangle-\left\langle i k \times \hat{B}, \hat{u}_{ \pm}\right\rangle-\left\langle\hat{u}_{+}, \hat{u}_{ \pm}\right\rangle+\left\langle\hat{u}_{-}, \hat{u}_{ \pm}\right\rangle+\left\langle\hat{u}_{ \pm}, \hat{E}\right\rangle+\alpha_{ \pm}\left\langle\hat{u}_{ \pm}, \hat{E}\right\rangle-\alpha_{ \pm}\left\langle\hat{u}_{\mp}, \hat{E}\right\rangle \\
& \mp\|\hat{E}\|^{2}+A_{ \pm} \gamma\langle-i k(i k \cdot \hat{E}), \hat{E}\rangle=0 \\
& \frac{d}{d t}\left\langle\hat{u}_{ \pm}, \hat{E}\right\rangle+A_{ \pm} \gamma\|k \cdot \hat{E}\|^{2} \mp\|\hat{E}\|^{2}=-\left\langle\hat{u}_{ \pm}, \hat{E}\right\rangle+\left\langle i k \times \hat{B}, \hat{u}_{ \pm}\right\rangle+\left\langle\hat{u}_{+}, \hat{u}_{ \pm}\right\rangle-\left\langle\hat{u}_{-}, \hat{u}_{ \pm}\right\rangle \\
& +\alpha_{ \pm}\left\langle\hat{u}_{ \pm}, \hat{E}\right\rangle-\alpha_{ \pm}\left\langle\hat{u}_{\mp}, \hat{E}\right\rangle,
\end{aligned}
$$

split the solution to two equations,

$$
\begin{aligned}
& \frac{d}{d t}\left\langle\hat{u}_{+}, \hat{E}\right\rangle+A_{+} \gamma\|k \cdot \hat{E}\|^{2}-\|\hat{E}\|^{2}=-\left\langle\hat{u}_{+}, \hat{E}\right\rangle+\left\langle i k \times \hat{B}, \hat{u}_{+}\right\rangle+\left\langle\hat{u}_{+}, \hat{u}_{+}\right\rangle-\left\langle\hat{u}_{-}, \hat{u}_{+}\right\rangle \\
& +\alpha_{+}\left\langle\hat{u}_{+}, \hat{E}\right\rangle-\alpha_{+}\left\langle\hat{u}_{-}, \hat{E}\right\rangle
\end{aligned}
$$


and

$$
\begin{aligned}
& \frac{d}{d t}\left\langle\hat{u}_{-}, \hat{E}\right\rangle+A_{-} \gamma\|k \cdot \hat{E}\|^{2}+\|\hat{E}\|^{2}=-\left\langle\hat{u}_{-}, \hat{E}\right\rangle+\left\langle i k \times \hat{B}, \hat{u}_{-}\right\rangle+\left\langle\hat{u}_{+}, \hat{u}_{-}\right\rangle-\left\langle\hat{u}_{-}, \hat{u}_{-}\right\rangle \\
& +\alpha_{-}\left\langle\hat{u}_{-}, \hat{E}\right\rangle-\alpha_{-}\left\langle\hat{u}_{+}, \hat{E}\right\rangle
\end{aligned}
$$

Now, taking the subtract of equation (3.13) from equation (3.12) we get

$$
\begin{aligned}
& \frac{d}{d t}\left\langle\hat{u}_{+}-\hat{u}_{-}, \hat{E}\right\rangle+\left(A_{+} \gamma-A_{-} \gamma\right)|k|^{2}\|\hat{E}\|^{2}-2\|\hat{E}\|^{2}=\left\langle\hat{u}_{-}-\hat{u}_{+}, \hat{E}\right\rangle \\
& +\left\langle i k \times \hat{B}, \hat{u}_{+}-\hat{u}_{-}\right\rangle+\left\langle\hat{u}_{+}, \hat{u}_{+}\right\rangle-\left\langle\hat{u}_{-}, \hat{u}_{+}\right\rangle+\left\|\left[\hat{u}_{+}, \hat{u}_{-}\right]\right\|^{2}-\left\langle\hat{u}_{-}, \hat{u}_{+}\right\rangle \\
& -\left\langle\hat{u}_{+}, \hat{u}_{-}\right\rangle+\alpha_{+}\left\langle\left(\hat{u}_{+}-\hat{u}_{-}\right), \hat{E}\right\rangle-\alpha_{-}\left\langle\left(\hat{u}_{+}-\hat{u}_{-}\right), \hat{E}\right\rangle .
\end{aligned}
$$

Therefore, taking the real part and using the Cauchy-Schwarz inequality implies $\frac{d}{d t} R\left\langle\hat{u}_{+}-\hat{u}_{-}, \hat{E}\right\rangle+\lambda\left(|k|^{2}\|\hat{E}\|^{2}-2\|\hat{E}\|^{2}\right) \leq c\left\|\hat{u}_{-}-\hat{u}_{+}\right\|^{2}-R\left\langle i k \times \hat{B}, \hat{u}_{+}-\hat{u}_{-}\right\rangle+c\left\|\left[\hat{u}_{+}, \hat{u}_{-}\right]\right\|$.

Now, multiply by $\frac{|k|^{2}}{\left(1+|k|^{2}\right)^{2}}$

$$
\begin{aligned}
& \frac{d}{d t} \frac{|k|^{2} R\left\langle\left(\hat{u}_{+}-\hat{u}_{-}\right), \hat{E}\right\rangle}{\left(1+|k|^{2}\right)^{2}}+\frac{\lambda|k|^{2}\left(|k|^{2}\|\hat{E}\|^{2}+2\|\hat{E}\|^{2}\right)}{\left(1+|k|^{2}\right)^{2}} \leq c\left\|\hat{u}_{-}-\hat{u}_{+}\right\|^{2} \\
& -\frac{R|k|^{2}\left\langle i k \times \hat{B}, \hat{u}_{+}-\hat{u}_{-}\right\rangle}{\left(1+|k|^{2}\right)^{2}}+c\left\|\left[\hat{u}_{+}, \hat{u}_{-}\right]\right\|^{2}
\end{aligned}
$$

Similarly, multiply the third equation of the system (3.6) by $-i k \times \hat{B}$ and using the integration by parts.

$$
\left\langle-i k \times \hat{B}, \partial_{t} \hat{E}\right\rangle-\langle-i k \times \hat{B}, i k \times \hat{B}\rangle-\left\langle-i k \times \hat{B}, \hat{u}_{-}\right\rangle+\left\langle-i k \times \hat{B}, \hat{u}_{+}\right\rangle=0 .
$$

Since

$$
\left\langle-i k \times \hat{B}, \partial_{t} \hat{E}\right\rangle=\frac{d}{d t}\langle-i k \times \hat{B}, \hat{E}\rangle-\langle\hat{E},-i k \times \hat{B}\rangle
$$

Thus,

$$
\frac{d}{d t}\langle-i k \times \hat{B}, \hat{E}\rangle-\left\langle\hat{E},-i k \times \partial_{t} \hat{B}\right\rangle+|k \times \hat{B}|^{2}+\left\langle i k \times \hat{B}, \hat{u}_{-}\right\rangle-\left\langle i k \times \hat{B}, \hat{u}_{+}\right\rangle=0,
$$

then, replace $\partial_{t} \hat{B}$ by $-i k \times \hat{E}$. Thus,

$$
\frac{d}{d t}\langle-i k \times \hat{B}, \hat{E}\rangle+\|k \times \hat{B}\|^{2}=\|k \times \hat{E}\|^{2}-\left\langle i k \times \hat{B}, \hat{u}_{-}-\hat{u}_{+}\right\rangle .
$$


Now, taking the real part and using the Cauchy-Schwarz and dividing by $\left(1+|k|^{2}\right)^{2}$ implies

$$
\frac{d}{d t} \frac{R\langle-i k \times \hat{B}, \hat{E}\rangle}{\left(1+|k|^{2}\right)^{2}}+\frac{\lambda\|k \times \hat{B}\|^{2}}{\left(1+|k|^{2}\right)^{2}} \leq \frac{\|k \times \hat{E}\|^{2}}{\left(1+|k|^{2}\right)^{2}}+C\left\|\hat{u}_{-}-\hat{u}_{+}\right\|^{2}
$$

Finally, letting us define

$$
\begin{aligned}
\mathcal{E}(\hat{U}(t, k)) & =\left\|\left[\sqrt{A_{+} \gamma} \hat{\rho}_{+}, \sqrt{A_{-} \gamma} \hat{\rho}_{-}, \hat{u}_{+}, \hat{u}_{-}, \hat{E}, \hat{B}\right]\right\|^{2}+k_{1}\left(\frac{R\left\langle\hat{u}_{+}, i k \hat{\rho}_{+}\right\rangle}{1+|k|^{2}}+\frac{R\left\langle\hat{u}_{-}, i k \hat{\rho}_{-}\right\rangle}{1+|k|^{2}}\right) \\
& +k_{2} \frac{|k|^{2} R\left\langle\left(\hat{u}_{+}-\hat{u}_{-}\right), \hat{E}\right\rangle}{\left(1+|k|^{2}\right)^{2}}+k_{3} \frac{R\langle-i k \times \hat{B}, \hat{E}\rangle}{\left(1+|k|^{2}\right)^{2}} .
\end{aligned}
$$

We chosen the constants such that $0<k_{3} \ll k_{2} \ll k_{1} \ll 1$ and let $0<k_{j} \ll 1, i=1,2,3$ be small enough such that (3.4) holds. However, letting $0<k_{3} \ll k_{2} \ll k_{1} \ll 1$ with $k_{2}^{3 / 2} \ll k_{3}$ be further small enough.

Now, taking the summation of (3.6), (3.11) $\times k_{1},(3.14) \times k_{2}$ and (3.15) $\times k_{3}$ we get

$$
\begin{aligned}
& \frac{1}{2} \frac{d}{d t}\left\|\left[\sqrt{A_{+} \gamma} \hat{\rho}_{+}, \sqrt{A_{-} \gamma} \hat{\rho}_{-}, \hat{u}_{+}, \hat{u}_{-}, \hat{E}, \hat{B}\right]\right\|^{2}+C\left\|\left[\hat{u}_{+}, \hat{u}_{-}\right]\right\|^{2} \\
& +\frac{d}{d t} \frac{R\left\langle\hat{u}_{+}, i k \hat{\rho}_{+}\right\rangle}{1+|k|^{2}}+\frac{d}{d t} \frac{R\left\langle\hat{u}_{-}, i k \hat{\rho}_{-}\right\rangle}{1+|k|^{2}}+\frac{\lambda|k|^{2}\left(\left\|\hat{\rho}_{+}\right\|^{2}+\left\|\hat{\rho}_{-}\right\|^{2}\right)}{1+|k|^{2}}+\frac{\lambda\left\|\hat{\rho}_{-}-\hat{\rho}_{+}\right\|^{2}}{1+|k|^{2}}+ \\
& \frac{d}{d t} \frac{|k|^{2} R\left\langle\left(\hat{u}_{+}-\hat{u}_{-}\right), \hat{E}\right\rangle}{\left(1+|k|^{2}\right)^{2}}+\frac{\lambda|k|^{2}\left(|k|^{2}\|\hat{E}\|^{2}-2\|\hat{E}\|^{2}\right)}{\left(1+|k|^{2}\right)^{2}}+\frac{d}{d t} \frac{R\langle-i k \times \hat{B}, \hat{E}\rangle}{\left(1+|k|^{2}\right)^{2}} \\
& +\frac{\lambda\|k \times \hat{B}\|^{2}}{\left(1+|k|^{2}\right)^{2}} \leq \frac{C|k|^{2}\left\|\left[\hat{u}_{+}, \hat{u}_{-}\right]\right\|^{2}}{\left(1+|k|^{2}\right)^{2}}+C\left\|\hat{u}_{-}-\hat{u}_{+}\right\|^{2}-\frac{R|k|^{2}\left\langle i k \times \hat{B}, \hat{u}_{+}-\hat{u}_{-}\right\rangle}{\left(1+|k|^{2}\right)^{2}} \\
& +C\left\|\left[\hat{u}_{+}, \hat{u}_{-}\right]\right\|^{2}+\frac{\|k \times \hat{E}\|^{2}}{\left(1+|k|^{2}\right)^{2}}+C\left\|\hat{u}_{-}-\hat{u}_{+}\right\|^{2} .
\end{aligned}
$$

Thus,

$$
\frac{d}{d t} \varepsilon(\hat{U}(t, k))+\lambda\left\|\left[\hat{\rho}_{+}, \hat{\rho}_{-},\left(\hat{\rho}_{-}-\hat{\rho}_{+}\right), \hat{u}_{+}, \hat{u}_{-}\right]\right\|^{2}+\frac{\lambda|k|^{2}\|[\hat{E}, \hat{B}]\|^{2}}{\left(1+|k|^{2}\right)^{2}} \leq 0,
$$

where we use the identity $\|k \times \hat{B}\|^{2}=|k|^{2}\|\hat{B}\|^{2}$ due to $k \cdot \hat{B}=0$. In addition, by using the Cauchy-Schwarz inequality we obtain

$$
\frac{k_{2}|k|^{2} R\left\langle i k \times \hat{B},\left(\hat{u}_{+}-\hat{u}_{-}\right)\right\rangle}{\left(1+|k|^{2}\right)^{2}} \leq \frac{k_{2}^{1 / 2}|k|^{4}\left\|\left[\hat{u}_{+}, \hat{u}_{-}\right]\right\|^{2}}{2\left(1+|k|^{2}\right)^{2}}+\frac{k_{2}^{3 / 2}|k|^{2}\|\hat{B}\|^{2}}{2\left(1+|k|^{2}\right)^{2}} .
$$

Thus, from (3.16), since $\mathcal{E}(\hat{U}(t, k)) \sim|\hat{U}|^{2}$ and,

$$
\left\|\left[\hat{\rho}_{+}, \hat{\rho}_{-},\left(\hat{\rho}_{-}-\hat{\rho}_{+}\right), \hat{u}_{+}, \hat{u}_{-}\right]\right\|^{2}+\frac{|k|^{2}\|[\hat{E}, \hat{B}]\|^{2}}{\left(1+|k|^{2}\right)^{2}} \geq \frac{\lambda|k|^{2}|\hat{U}|^{2}}{\left(1+|k|^{2}\right)^{2}},
$$

we conclude (3.5), and this is the end of the proof. 


\subsection{Representation of solution}

Denote by $U=\left[\rho_{ \pm}, u_{ \pm}, E, B\right]=e^{t L} U_{0}$ the explicit solution to the Cauchy proplem (3.1), (3.2) satisfying (3.3). In this subsection, we study the representation of $U$.

First, we take the time derivative for the first equation and the divergance of the second equation of the system (3.1) and substitute $\nabla \cdot E=\rho_{+}-\rho_{-}$. So,

$$
\begin{gathered}
\partial_{t t} \rho_{ \pm}+\partial_{t} \nabla \cdot u_{ \pm}=0 \\
\nabla \cdot \partial_{t} u_{ \pm}+\nabla \cdot u_{ \pm}+\alpha_{ \pm} \nabla \cdot\left(u_{ \pm}-u_{\mp}\right) \mp \nabla \cdot E+A_{ \pm} \gamma \Delta \rho_{ \pm}=0 .
\end{gathered}
$$

From equation (3.17) we know $\partial_{t} \nabla \cdot u_{ \pm}=-\partial_{t t} \rho_{ \pm}$, and substituting this result into equation (3.18) and multiplying by $(-1)$ we get

$$
\partial_{t t} \rho_{ \pm}+\left(1+\alpha_{ \pm}\right) \partial_{t} \rho_{ \pm}-\alpha_{ \pm} \partial_{t} \rho_{\mp} \pm\left(\rho_{+}-\rho_{-}\right)-A_{ \pm} \gamma \Delta \rho_{ \pm}=0,
$$

with initial data given by

$$
\left\{\begin{array}{l} 
\pm\left.\left(\rho_{+}-\rho_{-}\right)\right|_{t=0}= \pm\left(\rho_{+0}-\rho_{-0}\right)= \pm \nabla \cdot E_{0}, \\
\left.\partial_{t} \rho_{ \pm}\right|_{t=0}=-\nabla \cdot u_{ \pm 0} .
\end{array}\right.
$$

Then, taking the Fourier transform of the second order ODE (3.19) with (3.20), we obtain

$$
\left\{\begin{array}{l}
\partial_{t t} \hat{\rho}_{ \pm}+\left(1+\alpha_{ \pm}\right) \partial_{t} \hat{\rho}_{ \pm}-\alpha_{ \pm} \partial_{t} \hat{\rho}_{\mp} \pm\left(\hat{\rho}_{+}-\hat{\rho}_{-}\right)+A_{ \pm} \gamma|k|^{2} \hat{\rho}_{ \pm}=0 \\
\pm\left.\left(\hat{\rho}_{+}-\hat{\rho}_{-}\right)\right|_{t=0}= \pm\left(\hat{\rho}_{+0}-\hat{\rho}_{-0}\right)= \pm i k \cdot \hat{E}_{0} \\
\left.\partial_{t} \hat{\rho}_{ \pm}\right|_{t=0}=-i k \cdot \hat{u}_{ \pm 0}
\end{array}\right.
$$

Now set

$$
\begin{aligned}
& x_{1}=\hat{\rho}_{+} \\
& x_{2}=\dot{x}_{1}=\partial_{t} \hat{\rho}_{+}, \\
& x_{3}=\hat{\rho}_{-}, \\
& x_{4}=\dot{x}_{3}=\partial_{t} \hat{\rho}_{-}, x=\left[x_{1}, x_{2}, x_{3}, x_{4}\right]^{T}
\end{aligned}
$$

Then

$$
x_{t}=A(k) x
$$

where

$$
A(k)=\left[\begin{array}{cccc}
0 & 1 & 0 & 0 \\
-1-A_{+} \gamma|k|^{2} & -\left(1+\alpha_{+}\right) & 1 & \alpha_{+} \\
0 & 0 & 0 & 1 \\
1 & \alpha_{-} & -1-A_{-} \gamma|k|^{2} & -\left(1+\alpha_{-}\right)
\end{array}\right]
$$


Note the eigenvector of the matrix $A(k)$ is given by

$$
a_{1}(\lambda)=\left[\begin{array}{c}
\left(1+A_{-} \gamma|k|^{2}\right)+\left(1+\alpha_{-}\right) \lambda+\lambda^{2} \\
{\left[\left(1+A_{-} \gamma|k|^{2}\right)+\left(1+\alpha_{-}\right) \lambda+\lambda^{2}\right] \lambda} \\
1+\alpha_{-} \lambda \\
\left(1+\alpha_{-} \lambda\right) \lambda
\end{array}\right]
$$

and

$$
a_{2}(\lambda)=\left[\begin{array}{c}
1+\alpha_{+} \lambda \\
\left(1+\alpha_{+} \lambda\right) \lambda \\
\left(1+A_{+} \gamma|k|^{2}\right)+\left(1+\alpha_{+}\right) \lambda+\lambda^{2} \\
{\left[\left(1+A_{+} \gamma|k|^{2}\right)+\left(1+\alpha_{+}\right) \lambda+\lambda^{2}\right] \lambda}
\end{array}\right]
$$

In next two subsections we provide an estimate for $\hat{u}_{ \pm}, \hat{\rho}_{ \pm}, \hat{E}, \hat{B}$. In the subsection of parallel part we estimate for $\hat{u}_{ \pm}, \hat{\rho}_{ \pm}$, and in the subsection of perpendicular part we estimate for $\hat{u}_{ \pm}, \hat{E}, \hat{B}$. To do so, we set $\tilde{k}=k /|k|$ and we use the relation $\hat{u}_{ \pm}=$ $\tilde{k} \tilde{k} \cdot \hat{u}_{ \pm}-\tilde{k} \times\left(\tilde{k} \times \hat{u}_{ \pm}\right)$where we refer to $\tilde{k} \tilde{k} \cdot \hat{u}_{ \pm}$as the "parallel part" and $\tilde{k} \times\left(\tilde{k} \times \hat{u}_{ \pm}\right)$ as the "perpendicular part."

\subsubsection{Parallel Part}

We proceed with asymptotic expansion of eigenvalues: let $\lambda_{j}(k), j=1,2,3,4$, be the eigenvalues of the matrix $A(k)$. Taking the determininant, we see the eigenvalues satisfy,

$$
\begin{aligned}
\operatorname{det}(A(k)-\lambda I) & =\lambda^{4}+\left(2+\alpha_{+}+\alpha_{-}\right) \lambda^{3}+\left(3+\alpha_{+}+\alpha_{-}+A_{+} \gamma|k|^{2}+A_{-} \gamma|k|^{2}\right) \lambda^{2} \\
& +\left(2+A_{+} \gamma|k|^{2}+A_{-} \gamma|k|^{2}+\alpha_{-} A_{+} \gamma|k|^{2}+\alpha_{+} A_{-} \gamma|k|^{2}\right) \lambda \\
& +A_{+} \gamma|k|^{2}+A_{-} \gamma|k|^{2}+A_{-} A_{+} \gamma^{2}|k|^{4}=0 .
\end{aligned}
$$

(i) First we consider when $|k| \rightarrow 0$, since $\operatorname{det}(A-\lambda I)=0$ satisfies

$$
\lambda(\lambda+1)\left(\lambda^{2}+\left(1+\alpha_{-}+\alpha_{+}\right) \lambda+2\right)=0,
$$

and has the following asymptotic expansion:

$$
\lambda_{j}(k)=\lambda_{j}^{(0)}+\lambda_{j}^{(1)} k+\lambda_{j}^{(2)} k^{2}+\ldots,
$$


where each coefficient $\lambda_{j}^{(n)}$ is given by direct computation as

$$
\begin{aligned}
& \lambda_{1}^{(0)}=\lambda_{1}^{(1)}=0, \quad \lambda_{1}^{(2)}=-\frac{1}{2}\left(A_{+} \gamma+A_{-} \gamma\right), \\
& \lambda_{2}^{(0)}=-1, \lambda_{2}^{(1)}=0, \lambda_{2}^{(2)}=-\frac{A_{+} \gamma+A_{-} \gamma+\alpha_{-} A_{+} \gamma+\alpha_{+} A_{-} \gamma}{2+\alpha_{+}+\alpha_{-}}, \\
& \lambda_{3}^{(0)}=\frac{-\left(1+\alpha_{+}-\alpha_{-}\right)-\sqrt{\left(1+\alpha_{+}+\alpha_{-}\right)^{2}-8}}{2}, \quad \lambda_{3}^{(1)}=0, \lambda_{3}^{(2)}=0, \\
& \lambda_{4}^{(0)}=\frac{-\left(1+\alpha_{+}-\alpha_{-}\right)+\sqrt{\left(1+\alpha_{+}+\alpha_{-}\right)^{2}-8}}{2}, \quad \lambda_{4}^{(1)}=0, \lambda_{4}^{(2)}=0 .
\end{aligned}
$$

Thus the approximation of the eigenvalue when $|k| \rightarrow 0$ is

$$
\begin{aligned}
& \lambda_{1}=-\frac{1}{2}\left(A_{+} \gamma+A_{-} \gamma\right) k^{2}, \\
& \lambda_{2}=-1-\left[\frac{A_{+} \gamma+A_{-} \gamma+\alpha_{-} A_{+} \gamma+\alpha_{+} A_{-} \gamma}{2-\alpha_{+}-\alpha_{-}}\right] k^{2}, \\
& \lambda_{3}=\frac{-\left(1+\alpha_{+}+\alpha_{-}\right)-\sqrt{\left(1+\alpha_{+}+\alpha_{-}\right)^{2}-8}}{2}, \\
& \lambda_{4}=\frac{-\left(1+\alpha_{+}+\alpha_{-}\right)+\sqrt{\left(1+\alpha_{+}+\alpha_{-}\right)^{2}-8}}{2} .
\end{aligned}
$$

Therefore, if we define

$$
B(t)=\left[\begin{array}{llll}
a_{1}\left(\lambda_{1}\right) e^{\lambda_{1} t} & a_{1}\left(\lambda_{2}\right) e^{\lambda_{2} t} & a_{2}\left(\lambda_{3}\right) e^{\lambda_{3} t} & a_{2}\left(\lambda_{4}\right) e^{\lambda_{4} t}
\end{array}\right],
$$

the Green matrix $G$ for (3.22) is given by $G(t)=B(t) B^{-1}(0)$ and the solution is

$$
x=G(t) x_{0} .
$$

Thus, after series of calculation using symbolic manipulater we have

$$
\left[\begin{array}{l}
x_{1} \\
x_{2} \\
x_{3} \\
x_{4}
\end{array}\right]=\left[\begin{array}{cccc}
\left(O(1) e^{-\theta t}\right. & \left(O(1) e^{-\theta t}\right. & \left(O(1) e^{-\theta t}\right. & \left(O(1) e^{-\theta t}\right. \\
\left.+O(1) e^{-\theta k^{2} t}\right) & \left.+O(1) e^{-\theta k^{2} t}\right) & \left.+O(1) e^{-\theta k^{2} t}\right) & \left.+O(1) e^{-\theta k^{2} t}\right) \\
\left(O(1) e^{-\theta t}\right. & \left(O(1) e^{-\theta t}\right. & \left(O(1) e^{-\theta t}\right. & \left(O(1) e^{-\theta t}\right. \\
\left.+O(1) e^{-\theta k^{2} t} k^{2}\right) & \left.+O(1) e^{-\theta k^{2} t} k^{2}\right) & \left.+O(1) e^{-\theta k^{2} t} k^{2}\right) & \left.+O(1) e^{-\theta k^{2} t} k^{2}\right) \\
\left(O(1) e^{-\theta t}\right. & \left(O(1) e^{-\theta t}\right. & \left(O(1) e^{-\theta t}\right. & \left(O(1) e^{-\theta t}\right. \\
\left.+O(1) e^{-\theta k^{2} t}\right) & \left.+O(1) e^{-\theta k^{2} t}\right) & \left.+O(1) e^{-\theta k^{2} t}\right) & \left.+O(1) e^{-\theta k^{2} t}\right) \\
\left(O(1) e^{-\theta t}\right. & \left(O(1) e^{-\theta t}\right. & \left(O(1) e^{-\theta t}\right. & \left(O(1) e^{-\theta t}\right. \\
\left.+O(1) e^{-\theta k^{2} t} k^{2}\right) & \left.+O(1) e^{-\theta k^{2} t} k^{2}\right) & \left.+O(1) e^{-\theta k^{2} t} k^{2}\right) & \left.+O(1) e^{-\theta k^{2} t} k^{2}\right)
\end{array}\right]\left[\begin{array}{c}
x_{1,0} \\
\\
x_{2,0} \\
x_{3,0} \\
x_{4,0}
\end{array}\right]
$$


where $\theta$ is the sum of real parts of $\lambda_{j}, j=1,2,3,4$. Thus,

$$
\begin{aligned}
\hat{\rho}_{+}(t, k)=( & \left.O(1) e^{-\theta t}+O(1) e^{-\theta k^{2} t}\right) \hat{\rho}_{+0}-\left(O(1) e^{-\theta t}+O(1) e^{-\theta k^{2} t}\right) i k \cdot \hat{u}_{+0} \\
& +\left(O(1) e^{-\theta t}+O(1) e^{-\theta k^{2} t}\right) \hat{\rho}_{-0}-\left(O(1) e^{-\theta t}+O(1) e^{-\theta k^{2} t}\right) i k \cdot \hat{u}_{-0}, \\
-i k \cdot \hat{u}_{+}(t, k) & =\left(O(1) e^{-\theta t}+O(1) e^{-\theta k^{2} t} k^{2}\right) \hat{\rho}_{+0}-\left(O(1) e^{-\theta t}+O(1) e^{-\theta k^{2} t} k^{2}\right) i k \cdot \hat{u}_{+0} \\
& +\left(O(1) e^{-\theta t}+O(1) e^{-\theta k^{2} t} k^{2}\right) \hat{\rho}_{-0}-\left(O(1) e^{-\theta t}+O(1) e^{-\theta k^{2} t} k^{2}\right) i k \cdot \hat{u}_{-0}, \\
\hat{\rho}_{-}(t, k)=( & \left.O(1) e^{-\theta k^{2} t}+O(1) e^{-\theta t}\right) \hat{\rho}_{+0}-\left(O(1) e^{-\theta k^{2} t}+O(1) e^{-\theta t}\right) i k \cdot \hat{u}_{+0} \\
& +\left(O(1) e^{-\theta k^{2} t}+O(1) e^{-\theta t}\right) \hat{\rho}_{-0}-\left(O(1) e^{-\theta k^{2} t}+O(1) e^{-\theta t}\right) i k \cdot \hat{u}_{-0}, \\
-i k \cdot \hat{u}_{-}(t, k) & =\left(O(1) e^{-\theta t}+O(1) e^{-\theta k^{2} t} k^{2}\right) \hat{\rho}_{+0}-\left(O(1) e^{-\theta t}+O(1) e^{-\theta k^{2} t} k^{2}\right) i k \cdot \hat{u}_{+0} \\
& +\left(O(1) e^{-\theta t}+O(1) e^{-\theta k^{2} t} k^{2}\right) \hat{\rho}_{-0}-\left(O(1) e^{-\theta t}+O(1) e^{-\theta k^{2} t} k^{2}\right) i k \cdot \hat{u}_{-0} .
\end{aligned}
$$

In fact we know that

$$
\hat{u}_{ \pm}=\tilde{k} \tilde{k} \cdot \hat{u}_{ \pm}-\tilde{k} \times\left(\tilde{k} \times \hat{u}_{ \pm}\right) \text {implies } \tilde{k} \tilde{k} \cdot \hat{u}_{ \pm}=\hat{u}_{ \pm}+\tilde{k} \times\left(\tilde{k} \times \hat{u}_{ \pm}\right) .
$$

Therefore, plugging (3.26) in the second and the fourth equations of (3.25), we obtain

$$
\begin{aligned}
& \hat{u}_{+}(t, k)=-\tilde{k} \times\left(\tilde{k} \times \hat{u}_{+}\right)+\left(\frac{i \tilde{k}}{|k|}\left(O(1) e^{-\theta t}+O(1) e^{-\theta k^{2} t} k^{2}\right) \hat{\rho}_{+0}\right. \\
& +\left(O(1) e^{-\theta t}+O(1) e^{-\theta k^{2} t} k^{2}\right) \tilde{k} \tilde{k} \cdot \hat{u}_{+0}+\frac{i \tilde{k}}{|k|}\left(O(1) e^{-\theta t}+O(1) e^{-\theta k^{2} t} k^{2}\right) \hat{\rho}_{-0} \\
& \left.\quad+\left(O(1) e^{-\theta t}+O(1) e^{-\theta k^{2} t} k^{2}\right) \tilde{k} \tilde{k} \cdot \hat{u}_{-0}\right), \\
& \hat{u}_{-}(t, k)=-\tilde{k} \times\left(\tilde{k} \times \hat{u}_{-}\right)+\left(\frac{i \tilde{k}}{|k|}\left(O(1) e^{-\theta t}+O(1) e^{-\theta k^{2} t} k^{2}\right) \hat{\rho}_{+0}\right. \\
& \quad+\left(O(1) e^{-\theta t}+O(1) e^{-\theta k^{2} t} k^{2}\right) \tilde{k} \tilde{k} \cdot \hat{u}_{+0}+\frac{i \tilde{k}}{|k|}\left(O(1) e^{-\theta t}+O(1) e^{-\theta k^{2} t} k^{2}\right) \hat{\rho}_{-0} \\
& \left.\quad+\left(O(1) e^{-\theta t}+O(1) e^{-\theta k^{2} t} k^{2}\right) \tilde{k} \tilde{k} \cdot \hat{u}_{-0}\right) .
\end{aligned}
$$

(ii) When $|k| \rightarrow \infty, \lambda_{j}(k)$ has the following asymptotic expansion

$$
\lambda_{j}(k)=\mu_{j}^{(1)} k+\mu_{j}^{(0)}+\mu_{j}^{(-1)} k^{-1}+\mu_{j}^{(-2)} k^{-2}+\mu_{j}^{(-3)} k^{-3}+\ldots
$$


where each coefficient $\mu_{j}^{(n)}$ is given by direct computation as

$$
\begin{aligned}
& \mu_{1}^{(1)}=i \sqrt{A_{-} \gamma}, \quad \mu_{1}^{(0)}=-\frac{\left(1+\alpha_{-}\right)}{2}, \\
& \mu_{2}^{(1)}=-i \sqrt{A_{-} \gamma}, \quad \mu_{2}^{(0)}=-\frac{\left(1+\alpha_{-}\right)}{2}, \\
& \mu_{3}^{(1)}=i \sqrt{A_{+} \gamma}, \quad \mu_{3}^{(0)}=-\frac{\left(1+\alpha_{+}\right)}{2}, \\
& \mu_{4}^{(1)}=-i \sqrt{A_{+} \gamma}, \quad \mu_{4}^{(0)}=-\frac{\left(1+\alpha_{+}\right)}{2} .
\end{aligned}
$$

The approximation of the eigenvalue when $|k| \rightarrow \infty$ is

$$
\begin{aligned}
& \lambda_{1}=-\frac{\left(1+\alpha_{-}\right)}{2}+i \sqrt{A_{-} \gamma} k, \\
& \lambda_{2}=-\frac{\left(1+\alpha_{-}\right)}{2}-i \sqrt{A_{-} \gamma} k, \\
& \lambda_{3}=-\frac{\left(1+\alpha_{+}\right)}{2}+i \sqrt{A_{+} \gamma} k, \\
& \lambda_{4}=-\frac{\left(1+\alpha_{+}\right)}{2}-i \sqrt{A_{+} \gamma} k .
\end{aligned}
$$

So $G=B(t) B^{-1}(0)$ is the Green matrix for (3.22) and the solution is

$$
x=G(t) x_{0}
$$

Thus, after series of calculation using symbolic manipulater we obtain

$$
\begin{aligned}
& {\left[\begin{array}{l}
x_{1} \\
x_{2} \\
x_{3} \\
x_{4}
\end{array}\right]=\left[\begin{array}{cccc}
O(1) e^{-\theta t} & O(1) e^{-\theta t}|k|^{-1} & O(1) e^{-\theta t}|k|^{-1} & O(1) e^{-\theta t} \\
O(1) e^{-\theta t}|k| & O(1) e^{-\theta t} & O(1) e^{-\theta t} & O(1) e^{-\theta t}|k|^{-1} \\
O(1) e^{-\theta t}|k|^{-1} & O(1) e^{-\theta t} & O(1) e^{-\theta t} & O(1) e^{-\theta t}|k|^{-1} \\
O(1) e^{-\theta t} & O(1) e^{-\theta t}|k|^{-1} & O(1) e^{-\theta t}|k| & O(1) e^{-\theta t}
\end{array}\right]\left[\begin{array}{c}
x_{1,0} \\
x_{2,0} \\
x_{3,0} \\
x_{4,0}
\end{array}\right] .} \\
& \hat{\rho}_{+}(t, k)=O(1) e^{-\theta t} \hat{\rho}_{+0}-O(1) e^{-\theta t}|k|^{-1} i k \cdot \hat{u}_{+0}+O(1) e^{-\theta t}|k|^{-1} \hat{\rho}_{-0}-O(1) e^{-\theta t} i k \cdot \hat{u}_{-0}, \\
& -i k \cdot \hat{u}_{+}(t, k)=O(1) e^{-\theta t}|k| \hat{\rho}_{+0}-O(1) e^{-\theta t} i k \cdot \hat{u}_{+0}+O(1) e^{-\theta t} \hat{\rho}_{-0}-O(1) e^{-\theta t}|k|^{-1} i k \cdot \hat{u}_{-0} \text {, } \\
& \hat{\rho}_{-}(t, k)=O(1) e^{-\theta t}|k|^{-1} \hat{\rho}_{+0}-O(1) e^{-\theta t} i k \cdot \hat{u}_{+0}+O(1) e^{-\theta t} \hat{\rho}_{-0}-O(1) e^{-\theta t}|k|^{-1} i k \cdot \hat{u}_{-0}, \\
& -i k \cdot \hat{u}_{-}(t, k)=O(1) e^{-\theta t} \hat{\rho}_{+0}-O(1) e^{-\theta t}|k|^{-1} i k \cdot \hat{u}_{+0}+O(1) e^{-\theta t}|k| \hat{\rho}_{-0}-O(1) e^{-\theta t} i k \cdot \hat{u}_{-0} .
\end{aligned}
$$


Therefore, after plugging (3.26) into the second and fourth equations of (3.29), we obtain

$$
\begin{gathered}
\hat{u}_{+}(t, k)=-\tilde{k} \times\left(\tilde{k} \times \hat{u}_{+}\right)+\left(\begin{array}{c}
O \\
(1) e^{-\theta t} i \tilde{k} \tilde{k} \cdot \hat{\rho}_{+0}+O(1) e^{-\theta t} \tilde{k} \tilde{k} \cdot \hat{u}_{+0}
\end{array}\right. \\
\left.+O(1) e^{-\theta t} \frac{i \tilde{k}}{|k|} \hat{\rho}_{-0}+O(1) e^{-\theta t} \frac{\tilde{k} \tilde{k}}{|k|} \cdot \hat{u}_{-0}\right) \\
\hat{u}_{-}(t, k)=-\tilde{k} \times\left(\tilde{k} \times \hat{u}_{-}\right)+\left(\begin{array}{c}
O(1) e^{-\theta t} \frac{i \tilde{k}}{|k|} \hat{\rho}_{+0}+O(1) e^{-\theta t} \frac{\tilde{k} \tilde{k}}{|k|} \cdot \hat{u}_{+0} \\
\left.+O(1) e^{-\theta t} i \tilde{k} \tilde{k} \hat{\rho}_{-0}+O(1) e^{-\theta t} \tilde{k} \tilde{k} \cdot \hat{u}_{-0}\right)
\end{array}\right.
\end{gathered}
$$

(iii) When $0<|k|<\infty$, we consider the Routh-Hurwitz stability condition of the characteristic polynomial (3.23). That is, if we write (3.23) in the form

$$
b_{0} \lambda^{4}+b_{1} \lambda^{3}+b_{2} \lambda^{2}+b_{3} \lambda+b_{4}=0
$$

The system stability requires

$$
b_{1}>0, b_{1} b_{2}-b_{0} b_{3}>0,\left(b_{1} b_{2}-b_{0} b_{3}\right) b_{3}-b_{1}^{2} b_{4}>0, b_{4}>0 .
$$

It can be shown that, in our case, the conditions corresponding to (3.31) are given respectively, by

$$
\begin{aligned}
& \left(2+\alpha_{+}+\alpha_{-}\right)>0, \\
& {\left[\left(2+\alpha_{+}+\alpha_{-}\right)\left(3+\alpha_{+}+\alpha_{-}+A_{+} \gamma|k|^{2}+A_{-} \gamma|k|^{2}\right)\right]-\left(2+A_{+} \gamma|k|^{2}+A_{-} \gamma|k|^{2}+\alpha_{-} A_{+} \gamma|k|^{2}\right.} \\
& \left.+\alpha_{+} A_{-} \gamma|k|^{2}\right)>0, \\
& {\left[\left(4+2 \alpha_{+}+2 \alpha_{-}+A_{+} \gamma|k|^{2}+A_{-} \gamma|k|^{2}+3 \alpha_{+}+\alpha_{+}^{2}+2 \alpha_{+} \alpha_{-}+\alpha_{+} A_{+} \gamma|k|^{2}+3 \alpha_{-}+\alpha_{-}^{2}\right.\right.} \\
& \left.\left.+\alpha_{-} A_{-} \gamma|k|^{2}\right)\left(2+A_{+} \gamma|k|^{2}+A_{-} \gamma|k|^{2}+\alpha_{-} A_{+} \gamma|k|^{2}+\alpha_{+} A_{-} \gamma|k|^{2}\right)\right] \\
& -\left[\left(2+\alpha_{+}+\alpha_{-}\right)^{2}\left(A_{+} \gamma|k|^{2}+A_{-} \gamma|k|^{2}+A_{-} A_{+} \gamma^{2}|k|^{4}\right)\right]>0, \\
& A_{+} \gamma|k|^{2}+A_{-} \gamma|k|^{2}+A_{-} A_{+} \gamma^{2}|k|^{4}>0 .
\end{aligned}
$$

It is not difficult to show that above inequalities are satisfied and this implies all roots of the characteristic equation have negative real parts.

Although the eigenvalues may coalesce, the computations in (i), (ii), and (iii) show that coalescence occurs when the real parts of the eigenvalues are negative. Therefore, the stability conditions are satisfied. 


\subsubsection{Perpendicular Part}

Now we consider

$$
\left\{\begin{array}{l}
M_{1}(t, k):=-\tilde{k} \times\left(\tilde{k} \times \hat{u}_{+}(t, k)\right), \\
M_{2}(t, k):=-\tilde{k} \times\left(\tilde{k} \times \hat{u}_{-}(t, k)\right), \\
M_{3}(t, k):=-\tilde{k} \times(\tilde{k} \times \hat{E}(t, k)), \\
M_{4}(t, k):=-\tilde{k} \times(\tilde{k} \times \hat{B}(t, k)),
\end{array}\right.
$$

when $t>0$ and $|k| \neq 0$. Taking the curl of all but the first and the last equation in system (3.1) we have

$$
\left\{\begin{array}{l}
\partial_{t}\left(\nabla \times u_{+}\right)+\nabla \times u_{+}+\alpha_{+}\left(\nabla \times u_{+}\right)-\alpha_{+}\left(\nabla \times u_{-}\right)-\nabla \times E=0, \\
\partial_{t}\left(\nabla \times u_{-}\right)+\nabla \times u_{-}+\alpha_{-}\left(\nabla \times u_{-}\right)-\alpha_{-}\left(\nabla \times u_{+}\right)+\nabla \times E=0, \\
\partial_{t}(\nabla \times E)-\nabla \times(\nabla \times B)+\nabla \times u_{+}-\nabla \times u_{-}=0 \\
\partial_{t}(\nabla \times B)+\nabla \times(\nabla \times E)=0 .
\end{array}\right.
$$

Now, taking the Fourier Transform and multiplying by $-i k$, we obtain

$$
\left\{\begin{array}{l}
\partial_{t} M_{1}+M_{1}+\alpha_{+} M_{1}-\alpha_{+} M_{2}-M_{3}=0, \\
\partial_{t} M_{2}+M_{2}+\alpha_{-} M_{2}-\alpha_{-} M_{1}+M_{3}=0, \\
\partial_{t} M_{3}-i k \times M_{4}+M_{1}-M_{2}=0, \\
\partial_{t} M_{4}+i k \times M_{3}=0 .
\end{array}\right.
$$

Subtracting the first equation from the second equation in (3.33) we obtain

$$
\partial_{t}\left(M_{2}-M_{1}\right)+\left(M_{2}-M_{1}\right)+\alpha_{-}\left(M_{2}-M_{1}\right)+\alpha_{+}\left(M_{2}-M_{1}\right)+2 M_{3}=0,
$$

and we simplify the above computation by letting $M_{5}=M_{2}-M_{1}$.

So,

$$
\partial_{t} M_{5}+\left(1+\alpha_{+}+\alpha_{-}\right) M_{5}+2 M_{3}=0
$$

Thus,

$$
\left\{\begin{array}{l}
\partial_{t} M_{5}=-\left(1+\alpha_{+}+\alpha_{-}\right) M_{5}-2 M_{3} \\
\partial_{t} M_{3}=i k \times M_{4}+M_{5} \\
\partial_{t} M_{4}=-i k \times M_{3}
\end{array}\right.
$$

where we write the initial data in the form

$$
\left.\left[M_{3}, M_{4}, M_{5}\right]\right|_{t=0}=\left[M_{3,0}, M_{4,0}, M_{5,0}\right],
$$

with

$$
M_{5,0}=-\tilde{k} \times\left(\tilde{k} \times\left(\hat{u}_{-}-\hat{u}_{+}\right)\right), \quad M_{3,0}=-\tilde{k} \times\left(\tilde{k} \times \hat{E}_{0}\right), \quad M_{4,0}=-\tilde{k} \times\left(\tilde{k} \times \hat{B}_{0}\right) .
$$


Taking the time derivative of $\partial_{t} M_{3}$ and substituting $\partial_{t} M_{5}$ and $\partial_{t} M_{4}$ as given in (3.34), we get that

$$
\partial_{t t} M_{3}=k \times\left(k \times M_{3}\right)-\left(1+\alpha_{+}+\alpha_{-}\right) M_{5}-2 M_{3} .
$$

From $k \cdot M_{2}=0$, we find $k \times\left(k \times M_{3}\right)=-|k|^{2} M_{3}$ thus

$$
\partial_{t t} M_{3}+\left(2+|k|^{2}\right) M_{3}=-\left(1+\alpha_{+}+\alpha_{-}\right) M_{5} .
$$

Now taking the time derivative of (3.36) and replace $\partial_{t} M_{5}$ by the first equation of (3.34) and taking the sum with equation (3.36). Then

$$
\partial_{t t t} M_{3}+\left(1+\alpha_{+}+\alpha_{-}\right) \partial_{t t} M_{3}+\left(2+|k|^{2}\right) \partial_{t} M_{3}+\left(1+\alpha_{+}+\alpha_{-}\right)|k|^{2} M_{3}=0,
$$

with initial data

$$
\left\{\begin{array}{l}
\left.M_{3}\right|_{t=0}=M_{3,0} \\
\left.\partial_{t} M_{3}\right|_{t=0}=i k \times M_{4,0}+M_{5,0}, \\
\left.\partial_{t t} M_{3}\right|_{t=0}=-\left(1+\alpha_{+}+\alpha_{-}\right) M_{5,0}-\left(2+|k|^{2}\right) M_{3,0} .
\end{array}\right.
$$

Note that the characteristic equation of (3.37) is

$$
F(x):=\chi^{3}+\left(1+\alpha_{+}+\alpha_{-}\right) \chi^{2}+\left(2+|k|^{2}\right) \chi+\left(1+\alpha_{+}+\alpha_{-}\right)|k|^{2}=0 .
$$

The exact roots of the characteristic equation (3.39), along with their fundamental properties, are given by

Lemma 3.2. Let $|k| \neq 0$. The equation $F(\chi)=0, \chi \in \mathbb{C}$, has a real root $\eta=\eta(|k|)$ where $-\left(1+\alpha_{+}+\alpha_{-}\right)<\eta<0$ and two conjugate complex roots $\chi_{ \pm}=\beta \pm i \omega$ with $\beta=\beta(|k|) \in\left(-\frac{\left(\left(1+\alpha_{+}+\alpha_{-}\right)+\eta\right)}{4}, 0\right)$, and $\omega=\omega(|k|) \in\left(\frac{\sqrt{3\left(1+\alpha_{+}+\alpha_{-}\right)^{2}-6}}{3}, \infty\right)$ satisfying

$$
\begin{aligned}
& \beta=-\frac{\left(\left(1+\alpha_{+}+\alpha_{-}\right)+\eta\right)}{2}, \\
& \omega=\frac{\sqrt{-\left(1+\alpha_{+}+\alpha_{-}+\eta\right)\left(1+\alpha_{+}+\alpha_{-}-3 \eta\right)+4\left(2+|k|^{2}\right)}}{2}
\end{aligned}
$$

$\eta, \beta, \omega$ are smooth over $|k|>0$, and $\eta(|k|)$ is strictly decreasing in $|k|>0$ with

$$
\lim _{|k| \rightarrow 0} \eta(|k|)=0, \quad \lim _{|k| \rightarrow \infty} \eta(|k|)=-\left(1+\alpha_{+}+\alpha_{-}\right)
$$

Moreover, we have

$$
\begin{aligned}
& \eta(|k|)=-O(1)|k|^{2}, \quad \beta(|k|)=-\frac{\left(1+\alpha_{+}+\alpha_{-}\right)}{4}+O(1)|k|^{2}, \\
& \omega(|k|)=\frac{\sqrt{\left(1+\alpha_{+}+\alpha_{-}\right)\left(1+\alpha_{+}+\alpha_{-}\right)+8}}{4}+O(1)|k|
\end{aligned}
$$


whenever $|k| \leq\left(1+\alpha_{+}+\alpha_{-}\right)$is small, and

$$
\eta(|k|)=-\left(1+\alpha_{+}+\alpha_{-}\right)+O(1)|k|^{-2}, \quad \beta(|k|)=-O(1)|k|^{-2}, \quad \omega(|k|)=O(1)|k|
$$

whenever $|k| \geq\left(1+\alpha_{+}+\alpha_{-}\right)$is large.

Proof : Suppose $|k| \neq 0$. First, we determine if $F(\chi)=0$ admits a real root. We have

$$
\begin{aligned}
F^{\prime}(\chi) & =3 x^{2}+\left(1+\alpha_{+}+\alpha_{-}\right) x+2+|k|^{2}=3\left(x+\frac{1}{3}\left(1+\alpha_{+}+\alpha_{-}\right)^{2}-\frac{1}{3}\left(1+\alpha_{+}+\alpha_{-}\right)^{2}\right. \\
& +2+|k|^{2}>0
\end{aligned}
$$

Now $F(0)=\left(1+\alpha_{+}+\alpha_{-}\right)|k|^{2}>0$ and $F\left(-\left(1+\alpha_{+}+\alpha_{-}\right)\right)=-2\left(1+\alpha_{+}+\alpha_{-}\right)<0$, so $F(\chi)=0$ does in fact admit one real root which we will denote by $\eta=\eta(|k|)$. Note that $\eta$ satisfies $-\left(1+\alpha_{+}+\alpha_{-}\right)<\eta<0$. Since $F(\cdot)$ is smooth, $\eta(\cdot)$ is also smooth in $|k|>0$. Now, taking the derivative of $F(\eta(|k|))=0$ with respect to $|k|$ we obtain

$$
\eta^{\prime}(|k|)=\frac{-2|k|\left(\eta(|k|)+\left(1+\alpha_{+}+\alpha_{-}\right)\right)}{3[\eta(|k|)]^{2}+2\left(1+\alpha_{+}+\alpha_{-}\right) \eta(|k|)}+2+|k|^{2}<0 .
$$

Thus $\eta(\cdot)$ is strictly decreasing for $|k|>0$. Since $F(\eta)=0$ can be rewritten as

$$
\eta\left[\frac{\eta\left(\eta+\left(1+\alpha_{+}+\alpha_{-}\right)\right)}{2+|k|^{2}}+1\right]=-\frac{\left(1+\alpha_{+}+\alpha_{-}\right)|k|^{2}}{2+|k|^{2}},
$$

$\eta$ has limits 0 and $-\left(1+\alpha_{+}+\alpha_{-}\right)$as $|k| \rightarrow 0$ and $|k| \rightarrow \infty$ respectively. Furthermore $\eta=-O(1)|k|^{2}$ whenever $|k|<\left(1+\alpha_{+}+\alpha_{-}\right)$is small. $F(\eta)=0$ can also be written as

$$
\eta+\left(1+\alpha_{+}+\alpha_{-}\right)=\frac{-2 \eta}{\left(\eta^{2}+1\right)+|k|^{2}+2}=\frac{-2 \eta}{\eta^{2}+|k|^{2}+3}
$$

Therefore, $\eta=-\left(1+\alpha_{+}+\alpha_{-}\right)-O(1)|k|^{-2}$ when $|k| \geq\left(1+\alpha_{+}+\alpha_{-}\right)$is large.

Now, we will determine the roots of $F(\chi)=0$ over $\chi \in \mathbb{C}$. When $F(\eta)=0$ with $\eta \in \mathbb{R}, F(\chi)=0$ can be factored as

$$
F(\chi)=(\chi-\eta)\left[\chi^{2}+\left(1+\alpha_{+}+\alpha_{-}+\eta\right) \chi+\left(1+\alpha_{+}+\alpha_{-}+\eta\right) \eta+\left(2+|k|^{2}\right)\right]=0 .
$$

The two conjugate complex roots $\chi_{ \pm}=\beta \pm i \omega$ satisfy

$$
\chi^{2}+\left(1+\alpha_{+}+\alpha_{-}+\eta\right) \chi+\left(1+\alpha_{+}+\alpha_{-}+\eta\right) \eta+2+|k|^{2}=0 .
$$

By solving the above equation we obtained (3.40) for $\beta=\beta(|k|), \omega=\omega(|k|)$. Thus, the asymptotic behavior of $\beta=\beta(|k|), \omega=\omega(|k|)$ as $|k| \rightarrow 0$ and $|k| \rightarrow \infty$ is a consequence of $\eta(|k|)$. 
Now, from Lemma 3.2, we can write the solution of (3.37) as

$$
M_{3}(t, k)=c_{1}(k) e^{\eta t}+e^{\beta t}\left[c_{2}(k) \cos \omega t+c_{3}(k) \sin \omega t\right],
$$

where we determine $c_{i}(k)$ for each $i \in\{1,2,3\}$ later. By taking the first and second derivative of equation (3.41) with respect to $k$ we get

$$
\left[\begin{array}{c}
M_{3} \\
\partial_{t} M_{3} \\
\partial_{t t} M_{3}
\end{array}\right]_{t}=A\left[\begin{array}{l}
c_{1} \\
c_{2} \\
c_{3}
\end{array}\right], A:=\left[\begin{array}{ccc}
\mathbf{I}_{3} & \mathbf{I}_{3} & \mathbf{O}_{3} \\
\eta \mathbf{I}_{3} & \beta \mathbf{I}_{3} & \omega \mathbf{I}_{3} \\
\eta^{2} \mathbf{I}_{3} & \left(\beta^{2}-\omega^{2}\right) \mathbf{I}_{3} & 2 \beta \omega \mathbf{I}_{3}
\end{array}\right]
$$

The determinent of $A$ is

$\operatorname{det} A=\left[2 \beta \omega-\omega\left(\beta^{2}-\omega^{2}\right)\right]-\left[2 \eta \beta \omega-\eta^{2} \omega\right]=\omega\left[3 \eta^{2}+2\left(1+\alpha_{+}+\alpha_{-}\right) \eta+2+|k|^{2}\right]>0$, and

$$
A^{-1}=\frac{1}{\operatorname{det} A}\left[\begin{array}{ccc}
\left(\beta^{2}+\omega^{2}\right) \omega \mathbf{I}_{3} & -2 \beta \omega \mathbf{I}_{3} & \omega \mathbf{I}_{3} \\
\eta \omega(\eta 2 \beta) \mathbf{I}_{3} & 2 \beta \omega \mathbf{I}_{3} & -\omega \mathbf{I}_{3} \\
\eta\left(\beta^{2}-\omega^{2}-\eta \beta\right) \mathbf{I}_{3} & \left(\omega^{2}+\eta^{2}-\beta^{2}\right) \mathbf{I}_{3} & (\beta-\eta) \mathbf{I}_{3}
\end{array}\right]
$$

Therefore, by using the matrix of the initial data (3.38) we obtain

$$
\left[\begin{array}{l}
c_{1} \\
c_{2} \\
c_{3}
\end{array}\right]=A^{-1}\left[\begin{array}{ccc}
\mathbf{O}_{3} & \mathbf{I}_{3} & \mathbf{O}_{3} \\
\mathbf{I}_{3} & \mathbf{O}_{3} & i k \times \\
-\left(1+\alpha_{+}+\alpha_{-}\right) \mathbf{I}_{3} & -\left(2+|k|^{2}\right) \mathbf{I}_{3} & \mathbf{O}_{3}
\end{array}\right]\left[\begin{array}{l}
M_{5,0} \\
M_{3,0} \\
M_{4,0}
\end{array}\right]
$$

Substituting $\beta$ and $\omega$, it holds that

$$
\left[\begin{array}{l}
c_{1} \\
c_{2} \\
c_{3}
\end{array}\right]=\frac{1}{\operatorname{det} A}\left[\begin{array}{ccc}
-\left(2 \beta+\left(1+\alpha_{+}+\alpha_{-}\right)\right) \omega \mathbf{I}_{3} & \left(\beta^{2}+\omega^{2}-|k|^{2}-2\right) \omega \mathbf{I}_{3} & -2 \beta \omega i k \times \\
\left(2 \beta+\left(1+\alpha_{+}+\alpha_{-}\right)\right) \omega \mathbf{I}_{3} & \left(\eta^{2}-2 \eta \beta+|k|^{2}+2\right) \omega \mathbf{I}_{3} & 2 \beta \omega i k \times \\
{\left[\left(\omega^{2}+\eta^{2}-\beta^{2}\right)\right.} & {\left[\eta\left(\beta^{2}-\omega^{2}-\eta \beta\right)\right.} & \left(\omega^{2}+\eta^{2}\right. \\
\left.-(\beta-\eta)\left(1+\alpha_{+}+\alpha_{-}\right)\right] \mathbf{I}_{3} & \left.-(\beta-\eta)\left(|k|^{2}+2\right)\right] \mathbf{I}_{3} & \left.-\beta^{2}\right) i k \times
\end{array}\right]\left[\begin{array}{c}
M_{5,0} \\
M_{3,0} \\
M_{4,0}
\end{array}\right] .
$$

Thus,

$$
\left[c_{1}, c_{2}, c_{3}\right]^{T}=\frac{1}{3 \eta^{2}+2\left(1+\alpha_{+}+\alpha_{-}\right) \eta+2+|k|^{2}}
$$

$$
\left[\begin{array}{ccc}
\eta \mathbf{I}_{3} & \eta\left(1+\alpha_{+}+\alpha_{-}+\eta\right) \mathbf{I}_{3} & \left(1+\alpha_{+}+\alpha_{-}+\eta\right) i k \times \\
-\eta \mathbf{I}_{3} & \left(2 \eta^{2}+\left(1+\alpha_{+}+\alpha_{-}\right) \eta+|k|^{2}+2\right) \omega \mathbf{I}_{3} & -\left(1+\alpha_{+}+\alpha_{-}+\eta\right) i k \times \\
\frac{\frac{3}{2} \eta^{2}+\frac{3}{2} \eta\left(1+\alpha_{+}+\alpha_{-}\right)+\left(2+|k|^{2}\right)}{\omega} \mathbf{I}_{3} & \frac{\left(1+\alpha_{+}+\alpha_{-}+\eta\right)\left(\eta\left(1+\alpha_{+}+\alpha_{-}\right)+2+|k|^{2}\right)}{2 \omega} \mathbf{I}_{3} & \frac{-\left(1+\alpha_{+}+\alpha_{-}\right)^{2}}{2}+\frac{3}{2} \eta^{2}+\left(2+|k|^{2}\right) \\
\omega & &
\end{array}\right]\left[\begin{array}{c}
M_{5,0} \\
M_{3,0} \\
M_{4,0}
\end{array}\right],
$$


where

$$
\begin{aligned}
c_{1}= & \frac{1}{3 \eta^{2}+2\left(1+\alpha_{+}+\alpha_{-}\right) \eta+2+|k|^{2}}\left[\eta \mathbf{I}_{3} M_{5,0}(t, k)+\eta\left(1+\alpha_{+}+\alpha_{-}+\eta\right) \mathbf{I}_{3} M_{3,0}(t, k)\right. \\
& \left.+\left(1+\alpha_{+}+\alpha_{-}+\eta\right) i k \times M_{4,0}(t, k)\right] \\
c_{2}= & \frac{1}{3 \eta^{2}+2\left(1+\alpha_{+}+\alpha_{-}\right) \eta+2+|k|^{2}}\left[-\eta \mathbf{I}_{3} M_{5,0}(t, k)+\left(2 \eta+\eta\left(1+\alpha_{+}+\alpha_{-}+\eta\right)\right.\right. \\
& \left.\left.+|k|^{2}+2\right) \mathbf{I}_{3} M_{3,0}(t, k)-\left(1+\alpha_{+}+\alpha_{-}+\eta\right) i k \times M_{4,0}(t, k)\right] \\
c_{3}= & \frac{1}{3 \eta^{2}+2\left(1+\alpha_{+}+\alpha_{-}\right) \eta+2+|k|^{2}}\left[\frac{\frac{3}{2} \eta^{2}+\frac{3}{2} \eta\left(1+\alpha_{+}+\alpha_{-}\right)+\left(2+|k|^{2}\right)}{\omega} \mathbf{I}_{3} M_{5,0}(t, k)\right. \\
& +\frac{\left(1+\alpha_{+}+\alpha_{-}+\eta\right)\left(\eta\left(1+\alpha_{+}+\alpha_{-}\right)+2+|k|^{2}\right)}{2 \omega} \mathbf{I}_{3} M_{3,0}(t, k) \\
& \left.+\frac{\frac{-\left(1+\alpha_{+}+\alpha_{-}\right)^{2}}{2}+\frac{3}{2} \eta^{2}+\left(2+|k|^{2}\right)}{\omega} i k \times M_{4,0}(t, k)\right]
\end{aligned}
$$

Now, after substituting equation (3.41) in the first and the third equations of (3.34) and integrating in $t$ for both, it follows that

$$
\begin{gathered}
M_{5}(t, k)=M_{5,0}(k) e^{-t\left(1+\alpha_{+}+\alpha_{-}\right)}+2 e^{-t\left(1+\alpha_{+}+\alpha_{-}\right)}\left[\int_{0}^{t} c_{1}(k) e^{\left(1+\alpha_{+}+\alpha_{-}+\eta\right) s} d s\right. \\
\left.+\int_{0}^{t} e^{-t\left(1+\alpha_{+}+\alpha_{-}+\beta\right) s}\left(c_{2}(k) \cos \omega s+c_{3}(k) \sin \omega s\right) d s\right] \\
M_{4}(t, k)=M_{4,0}(k)-i k \times \int_{0}^{t} c_{1}(k) e^{\eta s}+e^{\beta s}\left(c_{2}(k) \cos \omega s+c_{3}(k) \sin \omega s\right) d s .
\end{gathered}
$$

Then we have

$$
\begin{aligned}
M_{5}(t, k)=[ & \left.M_{5,0}(k)+c_{4}(k)\right] e^{-t\left(1+\alpha_{+}+\alpha_{-}\right)}-\frac{2 c_{1}(k)}{\left(1+\alpha_{+}+\alpha_{-}+\eta\right)} e^{\eta t} \\
& +\frac{2 c_{2}(k)}{\left(1+\alpha_{+}+\alpha_{-}+\beta\right)^{2}+\omega^{2}} e^{\beta t}\left[\left(1+\alpha_{+}+\alpha_{-}+\beta\right) \cos \omega t+\omega \sin \omega t\right] \\
& +\frac{2 c_{3}(k)}{\left(1+\alpha_{+}+\alpha_{-}+\beta\right)^{2}+\omega^{2}} e^{\beta t}\left[\left(1+\alpha_{+}+\alpha_{-}+\beta\right) \sin \omega t-\omega \cos \omega t\right]
\end{aligned}
$$

and

$$
\begin{aligned}
M_{4}(t, k)=\left[M_{4,0}(k)+i k \times c_{5}(k)\right]-i k \times \frac{c_{1}(k)}{\eta} e^{\eta t} \\
-i k \times \frac{2 c_{2}(k)}{\beta^{2}+\omega^{2}} e^{\beta t}[\beta \cos \omega t+\omega \sin \omega t] \\
-i k \times \frac{2 c_{3}(k)}{\beta^{2}+\omega^{2}} e^{\beta t}[\beta \sin \omega t-\omega \cos \omega t]
\end{aligned}
$$


where $c_{4}(k), c_{5}(k)$ are chosen such that $\left.\left[M_{5}, M_{4}\right]\right|_{t=0}=\left[M_{5,0}, M_{4,0}\right]$ by (3.35). Thus

$$
\begin{aligned}
c_{4}(k)=- & \frac{2 c_{1}(k)}{\left(1+\alpha_{+}+\alpha_{-}+\eta\right)}-\frac{2 c_{2}(k)}{\left(1+\alpha_{+}+\alpha_{-}+\beta\right)^{2}+\omega^{2}}\left(1+\alpha_{+}+\alpha_{-}+\beta\right) \\
& \quad+\frac{2 c_{3}(k)}{\left(1+\alpha_{+}+\alpha_{-}+\beta\right)^{2}+\omega^{2}} \omega \\
c_{5}(k)= & \frac{c_{1}(k)}{\eta}+\frac{c_{2}(k)}{\beta^{2}+\omega^{2}} \beta-\frac{c_{3}(k)}{\beta^{2}+\omega^{2}} \omega .
\end{aligned}
$$

Therefore, substituting the first, second, and third equations of (3.44) into the above computations of (3.45) we have

$$
\begin{aligned}
& M_{5,0}(k)+c_{4}=0 \\
& M_{4,0}(k)+i k \times c_{5}=0 .
\end{aligned}
$$

Thus, for all $|k| \neq 0$

$$
\begin{aligned}
M_{5}(t, k)=- & \frac{2 c_{1}(k)}{\left(1+\alpha_{+}+\alpha_{-}+\eta\right)} e^{\eta t} \\
& +\frac{2 c_{2}(k)}{\left(1+\alpha_{+}+\alpha_{-}+\beta\right)^{2}+\omega^{2}} e^{\beta t}\left[\left(1+\alpha_{+}+\alpha_{-}+\beta\right) \cos \omega t+\omega \sin \omega t\right] \\
& +\frac{2 c_{3}(k)}{\left(1+\alpha_{+}+\alpha_{-}+\beta\right)^{2}+\omega^{2}} e^{\beta t}\left[\left(1+\alpha_{+}+\alpha_{-}+\beta\right) \sin \omega t-\omega \cos \omega t\right]
\end{aligned}
$$

and

$$
\begin{gathered}
M_{4}(t, k)=-i k \times \frac{c_{1}(k)}{\eta} e^{\eta t}-i k \times \frac{2 c_{2}(k)}{\beta^{2}+\omega^{2}} e^{\beta t}[\beta \cos \omega t+\omega \sin \omega t] \\
-i k \times \frac{2 c_{3}(k)}{\beta^{2}+\omega^{2}} e^{\beta t}[\beta \sin \omega t-\omega \cos \omega t] .
\end{gathered}
$$

Now set

$$
\begin{aligned}
& x_{1}=M_{3}, \\
& x_{2}=\dot{x}_{1}=\partial_{t} M_{3}, \\
& x_{3}=\dot{x}_{2}=\partial_{t t} M_{3}, \quad x=\left[x_{1}, x_{2}, x_{3}\right]^{T}
\end{aligned}
$$

so that

$$
\partial_{t t t} M_{3}=-\left(1+\alpha_{+}+\alpha_{-}\right) \partial_{t t} M_{3}-\left(2+|k|^{2}\right) \partial_{t} M_{3}-\left(1+\alpha_{+}+\alpha_{-}\right)|k|^{2} M_{3} .
$$


Then

$$
x_{t}=\Phi(k) x
$$

where

$$
\Phi(k)=\left[\begin{array}{ccc}
0 & 1 & 0 \\
0 & 0 & 1 \\
-\left(1+\alpha_{+}+\alpha_{-}\right)|k|^{2} & -\left(2+|k|^{2}\right) & -\left(1+\alpha_{+}+\alpha_{-}\right)
\end{array}\right]
$$

Asymptotic expansion of eigenvalues: Let $\lambda_{j}(k)$ be the eigenvalues of the matrix $\Phi(k)$. We will find the asymptotic expansion of the eigenvalues $\lambda_{j}(k)$ for $|k| \rightarrow$ 0 , and $|k| \rightarrow \infty$.

The eigenvalues $\lambda_{j}(k), j=1,2,3$, are the solutions of the characteristic equation

$\operatorname{det}(\Phi(k)-\lambda I)=\operatorname{det}\left[\begin{array}{ccc}-\lambda & 1 & 0 \\ 0 & -\lambda & 1 \\ -\left(1+\alpha_{+}+\alpha_{-}\right)|k|^{2} & -\left(2+|k|^{2}\right) & -\left(1+\alpha_{+}+\alpha_{-}\right)-\lambda\end{array}\right]=\left[\begin{array}{l}0 \\ 0 \\ 0\end{array}\right]$

which can be written

$$
\operatorname{det}(\Phi(k)-\lambda I)=\lambda^{3}+\left(1+\alpha_{+}+\alpha_{-}\right) \lambda^{2}+\left(2+|k|^{2}\right) \lambda+\left(1+\alpha_{+}+\alpha_{-}\right)|k|^{2}=0 .
$$

The eigenvector for $\Phi(k)$ is

$$
b(\lambda)=\left[\begin{array}{c}
1 \\
\lambda \\
\lambda^{2}
\end{array}\right]
$$

(i) When $|k| \rightarrow 0, \lambda_{j}(k)$ has the following asymptotic expansion

$$
\lambda_{j}(k)=\lambda_{j}^{(0)}+\lambda_{j}^{(1)} k+\lambda_{j}^{(2)} k^{2}+\ldots,
$$

where each coefficient $\lambda_{j}^{(n)}$ is given by direct computation as

$$
\begin{aligned}
& \lambda_{1}^{(0)}=\lambda_{1}^{(1)}=0, \quad \lambda_{1}^{(2)}=-\frac{1}{2}\left(1+\alpha_{+}+\alpha_{-}\right), \\
& \lambda_{2}^{(0)}=\frac{-\left(1+\alpha_{+}+\alpha_{-}\right)+\sqrt{\left(1+\alpha_{+}+\alpha_{-}\right)^{2}-8}}{2}, \quad \lambda_{2}^{(1)}=0, \\
& \lambda_{2}^{(2)}=\frac{-\left(1+\alpha_{+}+\alpha_{-}\right)-\sqrt{\left(1+\alpha_{+}+\alpha_{-}\right)^{2}-8}}{4}, \\
& \lambda_{3}^{(0)}=\frac{-\left(1+\alpha_{+}+\alpha_{-}\right)-\sqrt{\left(1+\alpha_{+}+\alpha_{-}\right)^{2}-8}}{2}, \quad \lambda_{3}^{(1)}=0, \\
& \lambda_{3}^{(2)}=\frac{-\left(1+\alpha_{+}+\alpha_{-}\right)+\sqrt{\left(1+\alpha_{+}+\alpha_{-}\right)^{2}-8}}{4} .
\end{aligned}
$$


Thus, the approximation of the eigenvalue when $|k| \rightarrow 0$ is

$$
\begin{aligned}
& \lambda_{1}=-\frac{1}{2}\left(1+\alpha_{+}+\alpha_{-}\right) k^{2}, \\
& \lambda_{2}=\frac{-\left(1+\alpha_{+}+\alpha_{-}\right)+\sqrt{\left(1+\alpha_{+}+\alpha_{-}\right)^{2}-8}}{2}-\left[\frac{\left(1+\alpha_{+}+\alpha_{-}\right)+\sqrt{\left(1+\alpha_{+}+\alpha_{-}\right)^{2}-8}}{4}\right] k^{2}, \\
& \lambda_{3}=\frac{-\left(1+\alpha_{+}+\alpha_{-}\right)-\sqrt{\left(1+\alpha_{+}+\alpha_{-}\right)^{2}-8}}{2}-\left[\frac{\left(1+\alpha_{+}+\alpha_{-}\right)-\sqrt{\left(1+\alpha_{+}+\alpha_{-}\right)^{2}-8}}{4}\right] k^{2} .
\end{aligned}
$$

Therefore, if we set

$$
D(t)=\left[\begin{array}{lll}
b\left(\lambda_{1}\right) e^{\lambda_{1} t} & b\left(\lambda_{2}\right) e^{\lambda_{2} t} & b\left(\lambda_{3}\right) e^{\lambda_{3} t}
\end{array}\right]
$$

the Green matrix $G$ for (3.48) is given by $G(t)=D(t) D^{-1}(0)$ and the solution is represented as

$$
x=G(t) x_{0},
$$

where each component of this solution is itself a $3 \times 3$ diagonal matrix. After series of calculation using symbolic manipulater and definition (3.32) the solution can be written as

$$
\begin{aligned}
&\left(-\tilde{k} \times\left(\tilde{k} \times \hat{u}_{-}\right)\right)-\left(-\tilde{k} \times\left(\tilde{k} \times \hat{u}_{+}\right)\right)=O(1) e^{-\theta k^{2} t}\left[\left(-\tilde{k} \times\left(\tilde{k} \times \hat{u}_{-0}\right)\right)-\left(-\tilde{k} \times\left(\tilde{k} \times \hat{u}_{+0}\right)\right)\right] \\
&+\left(O(1) e^{-\theta k^{2} t}-O(1) e^{-\theta t}\right)\left(-\tilde{k} \times\left(\tilde{k} \times \hat{E}_{0}\right)\right) \\
&+\left(O(1) e^{-\theta k^{2} t}-O(1) e^{-\theta t}-O(1) i e^{-\theta t}\right)\left(-\tilde{k} \times\left(\tilde{k} \times \hat{B}_{0}\right)\right), \\
&-\tilde{k} \times(\tilde{k} \times \hat{E})=\left(O(1) e^{-\theta t}-O(1) i e^{-\theta t}\right)\left(-\tilde{k} \times\left(\tilde{k} \times \hat{E}_{0}\right)\right)+\left(O(1) i e^{-\theta t}\right)\left(-\tilde{k} \times\left(\tilde{k} \times \hat{B}_{0}\right)\right), \\
&-\tilde{k} \times(\tilde{k} \times \hat{B})=\left(O(1) i e^{-\theta t}\right)\left(-\tilde{k} \times\left(\tilde{k} \times \hat{E}_{0}\right)\right)+\left(O(1) e^{-\theta t}-O(1) i e^{-\theta t}\right)\left(-\tilde{k} \times\left(\tilde{k} \times \hat{B}_{0}\right)\right) .
\end{aligned}
$$

By using equation (3.26), and substituting (3.27) in the first equation of (3.50), we obtain

$$
\begin{aligned}
\hat{u}_{-}-\hat{u}_{+} & =\frac{i \tilde{k}}{|k|}\left(O(1) e^{-\theta t}+O(1) e^{-\theta k^{2} t} k^{2}\right) \hat{\rho}_{+0}+\left(O(1) e^{-\theta t}+O(1) e^{-\theta k^{2} t} k^{2}\right) \tilde{k} \tilde{k} \cdot \hat{u}_{+0} \\
& +\frac{i \tilde{k}}{|k|}\left(O(1) e^{-\theta t}+O(1) e^{-\theta k^{2} t} k^{2}\right) \hat{\rho}_{-0}+\left(O(1) e^{-\theta t}+O(1) e^{-\theta k^{2} t} k^{2}\right) \tilde{k} \tilde{k} \cdot \hat{u}-0 \\
& +O(1) e^{-\theta k^{2} t}\left[\left(-\tilde{k} \times\left(\tilde{k} \times \hat{u}_{-0}\right)\right)-\left(-\tilde{k} \times\left(\tilde{k} \times \hat{u}_{+, 0}\right)\right)\right] \\
& +\left(O(1) e^{-\theta k^{2} t}-O(1) e^{-\theta t}\right)\left(-\tilde{k} \times\left(\tilde{k} \times \hat{E}_{0}\right)\right) \\
& +\left(O(1) e^{-\theta k^{2} t}-O(1) e^{-\theta t}-O(1) i e^{-\theta t}\right)\left(-\tilde{k} \times\left(\tilde{k} \times \hat{B}_{0}\right)\right) .
\end{aligned}
$$


Furthermore, we know from (3.1)

$$
\nabla \cdot E=\rho_{+}-\rho_{-},
$$

and thus

$$
\hat{E}=-\tilde{k} \times(\tilde{k} \times \hat{E})-\frac{i \tilde{k}}{|k|}\left(\hat{\rho}_{+}-\hat{\rho}_{-}\right) .
$$

Multiplying the first and the third equation of (3.25) by $\tilde{k}$ and substituting the result into equation (3.52), we obtain

$$
\begin{aligned}
\hat{E} & =\frac{-i \tilde{k}}{|k|}\left[\left(O(1) e^{-\theta t}+O(1) e^{-\theta k^{2} t}\right) \hat{\rho}_{+0}-\left(O(1) e^{-\theta t}+O(1) e^{-\theta k^{2} t}\right) i k \cdot \hat{u}_{+0}\right. \\
& \left.+\left(O(1) e^{-\theta t}+O(1) e^{-\theta k^{2} t}\right) \hat{\rho}_{-0}-\left(O(1) e^{-\theta t}+O(1) e^{-\theta k^{2} t}\right) i k \cdot \hat{u}_{-0}\right] \\
& +\left(O(1) e^{-\theta t}-O(1) i e^{-\theta t}\right)\left(-\tilde{k} \times\left(\tilde{k} \times \hat{E}_{0}\right)\right)+O(1) i e^{-\theta t}\left(-\tilde{k} \times\left(\tilde{k} \times \hat{B}_{0}\right)\right) .
\end{aligned}
$$

Now since $\tilde{k} \tilde{k} \cdot \hat{B}=0$, we have

$$
\hat{B}=\left(O(1) i e^{-\theta t}\right)\left(-\tilde{k} \times\left(\tilde{k} \times \hat{E}_{0}\right)\right)+\left(O(1) e^{-\theta t}-O(1) i e^{-\theta t}\right)\left(-\tilde{k} \times\left(\tilde{k} \times \hat{B}_{0}\right)\right) .
$$

Moreover, taking the sum of the first and the second equations of (3.33), we obtain

$$
\partial_{t}\left(M_{1}+M_{2}\right)+\left(M_{1}+M_{2}\right)+\left(M_{2}-M_{1}\right)\left(\alpha_{-}-\alpha_{+}\right)=0 .
$$

Thus,

$$
M_{1}+M_{2}=e^{-\theta t}\left(M_{1,0}+M_{2,0}\right)+\int_{0}^{t} e^{-(t-s)}\left(M_{2}-M_{1}\right)\left(\alpha_{-}-\alpha_{+}\right) d s .
$$

Substituting the first and the second equations of definition (3.32) into equation (3.54), we get that

$$
\begin{aligned}
\left(-\tilde{k} \times\left(\tilde{k} \times \hat{u}_{+}\right)\right)+ & \left(-\tilde{k} \times\left(\tilde{k} \times \hat{u}_{-}\right)\right)=e^{-\theta t}\left[\left(-\tilde{k} \times\left(\tilde{k} \times \hat{u}_{+0}\right)\right)+\left(-\tilde{k} \times\left(\tilde{k} \times \hat{u}_{-0}\right)\right)\right] \\
& +\int_{0}^{t} e^{-(t-s)}\left[O(1) e^{-\theta k^{2} s}\left[\left(-\tilde{k} \times\left(\tilde{k} \times \hat{u}_{-0}\right)\right)-\left(-\tilde{k} \times\left(\tilde{k} \times \hat{u}_{+0}\right)\right)\right]\right. \\
& +\left(O(1) e^{-\theta k^{2} s}-O(1) e^{-\theta s}\right)\left(-\tilde{k} \times\left(\tilde{k} \times \hat{E}_{0}\right)\right) \\
& \left.+\left(O(1) e^{-\theta k^{2} s}-O(1) e^{-\theta s}-O(1) i e^{-\theta s}\right)\left(-\tilde{k} \times\left(\tilde{k} \times \hat{B}_{0}\right)\right)\right] d s .
\end{aligned}
$$


Next, substituting equations (3.27) into the above computations, we obtain

$$
\begin{aligned}
\hat{u}_{+}(t, k)+ & \hat{u}_{-}(t, k)=\frac{i \tilde{k}}{|k|}\left(O(1) e^{-\theta t}+O(1) e^{-\theta k^{2} t} k^{2}\right) \hat{\rho}_{+0} \\
& +\left(O(1) e^{-\theta t}+O(1) e^{-\theta k^{2} t} k^{2}\right) \tilde{k} \tilde{k} \cdot \hat{u}_{+0} \\
& +\frac{i \tilde{k}}{|k|}\left(O(1) e^{-\theta t}+O(1) e^{-\theta k^{2} t} k^{2}\right) \hat{\rho}_{-0}+\left(O(1) e^{-\theta t}+O(1) e^{-\theta k^{2} t} k^{2}\right) \tilde{k} \tilde{k} \cdot \hat{u}_{-0} \\
& +e^{-\theta t}\left[\left(-\tilde{k} \times\left(\tilde{k} \times \hat{u}_{+0}\right)\right)+\left(-\tilde{k} \times\left(\tilde{k} \times \hat{u}_{-0}\right)\right)\right] \\
& +O(1)\left(e^{-\theta k^{2} t}-e^{-t}\right)\left[\left(-\tilde{k} \times\left(\tilde{k} \times \hat{u}_{+0}\right)\right)-\left(-\tilde{k} \times\left(\tilde{k} \times \hat{u}_{-0}\right)\right)\right] \\
& +O(1)\left(e^{-\theta k^{2} t}-e^{-t}\right)\left(-\tilde{k} \times\left(\tilde{k} \times \hat{E}_{0}\right)\right)+O(1)\left(e^{-\theta t}-e^{-t}\right)\left(-\tilde{k} \times\left(\tilde{k} \times \hat{E}_{0}\right)\right) \\
& +O(1)\left(e^{-\theta k^{2} t}-e^{-t}\right)\left(-\tilde{k} \times\left(\tilde{k} \times \hat{B}_{0}\right)\right)-O(1)\left(e^{-\theta t}-e^{-t}\right)\left(-\tilde{k} \times\left(\tilde{k} \times \hat{B}_{0}\right)\right) \\
& -O(1)\left(e^{-\theta t}-e^{-t}\right)\left(-\tilde{k} \times i\left(\tilde{k} \times \hat{B}_{0}\right)\right) .
\end{aligned}
$$

Now, taking the sum and difference respectively of the equations (3.51) and (3.56), we obtain

$$
\begin{aligned}
\hat{u}_{ \pm}(t, k)= & \frac{i \tilde{k}}{|k|}\left(O(1) e^{-\theta t}+O(1) e^{-\theta k^{2} t} k^{2}\right) \hat{\rho}_{+0}+\left(O(1) e^{-\theta t}+O(1) e^{-\theta k^{2} t} k^{2}\right) \tilde{k} \tilde{k} \cdot \hat{u}_{+0} \\
& +\frac{i \tilde{k}}{|k|}\left(O(1) e^{-\theta t}+O(1) e^{-\theta k^{2} t} k^{2}\right) \hat{\rho}_{-0}+\left(O(1) e^{-\theta t}+O(1) e^{-\theta k^{2} t} k^{2}\right) \tilde{k} \tilde{k} \cdot \hat{u}_{-0} \\
& +O(1) e^{-\theta k^{2} t}\left(-\tilde{k} \times\left(\tilde{k} \times \hat{u}_{-0}\right)\right)-\left(-\tilde{k} \times\left(\tilde{k} \times \hat{u}_{+0}\right)\right) \\
& +\left(O(1) e^{-\theta k^{2} t}-O(1) e^{-\theta t}\right)\left(-\tilde{k} \times\left(\tilde{k} \times \hat{E}_{0}\right)\right) \\
& +\left(O(1) e^{-\theta k^{2} t}-O(1) e^{-\theta t}-O(1) i e^{-\theta t}\right)\left(-\tilde{k} \times\left(\tilde{k} \times \hat{B}_{0}\right)\right) \\
& +\frac{i \tilde{k}}{|k|} O(1) e^{-\theta t} \hat{\rho}_{+0}+O(1) e^{-\theta t} \tilde{k} \tilde{k} \cdot \hat{u}_{+0}-\frac{i \tilde{k}}{|k|} O(1) e^{-\theta t} \hat{\rho}_{-0} \\
& +O(1) e^{-\theta t} \tilde{k} \tilde{k} \cdot \hat{u}_{-0}+e^{-\theta t}\left[\left(-\tilde{k} \times\left(\tilde{k} \times \hat{u}_{+0}\right)\right)+(-\tilde{k} \times(\tilde{k} \times \hat{u}-0))\right] \\
& +O(1) e^{-\theta k^{2} t}+O(1) e^{-\theta k^{2} t}-O(1) e^{-\theta t}+O(1) e^{-\theta k^{2} t}+O(1) e^{-\theta t} \\
& -O(1) i e^{-\theta t}-O(1) e^{-t} .
\end{aligned}
$$

(ii) When $|k| \rightarrow \infty, \lambda_{j}(k)$ has the following asymptotic expansion:

$$
\lambda_{j}(k)=\mu_{j}^{(1)} k+\mu_{j}^{(0)}+\mu_{j}^{(-1)} k^{-1}+\mu_{j}^{(-2)} k^{-2}+\ldots .
$$


Each coefficient $\mu_{j}^{(n)}$ is given by direct computation as

$$
\begin{array}{ll}
\mu_{1}^{(1)}=0, & \mu_{1}^{(0)}=-\left(1+\alpha_{+}+\alpha_{-}\right), \\
\mu_{2}^{(1)}=i, & \mu_{2}^{(0)}=0, \quad \mu_{2}^{(-1)}=\frac{-2}{3 i}, \quad \mu_{2}^{(-2)}=-\frac{4}{9} \\
\mu_{3}^{(1)}=-i, & \mu_{3}^{(0)}=0, \quad \mu_{3}^{(-1)}=\frac{2}{3 i}, \quad \mu_{3}^{(-2)}=-\frac{4}{9}
\end{array}
$$

The approximation of the eigenvalue when $|k| \rightarrow \infty$ is

$$
\begin{aligned}
& \lambda_{1}=-\left(1+\alpha_{+}+\alpha_{-}\right), \\
& \lambda_{2}=i k-\frac{2}{3 i} k^{-1}-\frac{4}{9} k^{-2}, \\
& \lambda_{3}=-i k+\frac{2}{3 i} k^{-1}-\frac{4}{9} k^{-2} .
\end{aligned}
$$

Hence, the Green matrix $G$ for (3.48) is given by $G(t)=D(t) D^{-1}(0)$ and the solution is represented as

$$
x=G(t) x_{0},
$$

where each component of this solution is itself a $3 \times 3$ diagonal matrix. After series of calculation using symbolic manipulater and equation (3.32) the solution can be written as

$$
\begin{aligned}
& \left(-\tilde{k} \times\left(\tilde{k} \times \hat{u}_{-}\right)\right)-\left(-\tilde{k} \times\left(\tilde{k} \times \hat{u}_{+}\right)\right) \\
& =O(1) e^{-\theta t}\left[\left(-\tilde{k} \times\left(\tilde{k} \times \hat{u}_{-, 0}\right)\right)-\left(-\tilde{k} \times\left(\tilde{k} \times \hat{u}_{+0}\right)\right)\right] \\
& +\frac{O(1)}{|k|} e^{-\theta k^{-2} t}\left(-\tilde{k} \times\left(\tilde{k} \times \hat{E}_{0}\right)\right) \\
& +\frac{O(1)}{|k|^{2}} e^{-\theta t}-\frac{O(1)}{|k|^{2}} e^{-\theta k^{-2} t}\left(-\tilde{k} \times\left(\tilde{k} \times \hat{B}_{0}\right)\right), \\
& -\tilde{k} \times(\tilde{k} \times \hat{E})=-O(1) e^{-\theta t}+O(1) e^{-\theta k^{-2} t}\left[\left(-\tilde{k} \times\left(\tilde{k} \times \hat{u}_{-0}\right)\right)-\left(-\tilde{k} \times\left(\tilde{k} \times \hat{u}_{+0}\right)\right]\right. \\
& +O(1) e^{-\theta k^{-2} t}\left(-\tilde{k} \times\left(\tilde{k} \times \hat{E}_{0}\right)\right)+\frac{O(1)}{|k|} e^{-\theta k^{-2} t}\left(-\tilde{k} \times\left(\tilde{k} \times \hat{B}_{0}\right)\right), \\
& -\tilde{k} \times(\tilde{k} \times \hat{B})=O(1) i|k| e^{-\theta k^{-2} t}\left[\left(-\tilde{k} \times\left(\tilde{k} \times \hat{u}_{-0}\right)\right)-\left(-\tilde{k} \times\left(\tilde{k} \times \hat{u}_{+0}\right)\right]\right. \\
& -O(1) i|k| e^{-\theta t}\left(-\tilde{k} \times\left(\tilde{k} \times \hat{E}_{0}\right)\right)+O(1) e^{-\theta k^{-2} t}\left(-\tilde{k} \times\left(\tilde{k} \times \hat{B}_{0}\right)\right) \text {. }
\end{aligned}
$$

By using (3.26), and substituting (3.30) into the first equation of the above computa- 
tion, we obtain

$$
\begin{aligned}
\hat{u}_{-}-\hat{u}_{+} & =\left(O(1) e^{-\theta t} \tilde{k} \tilde{k}-O(1) e^{-\theta t} \frac{i \tilde{k}}{|k|} i\right) \hat{\rho}_{+0}+\left(O(1) e^{-\theta t}-O(1) e^{-\theta t} \frac{1}{|k|}\right) \tilde{k} \tilde{k} \cdot \hat{u}_{+0} \\
& +\left(O(1) e^{-\theta t} \frac{i \tilde{k}}{|k|}-O(1) e^{-\theta t} i \tilde{k} \tilde{k}\right) \hat{\rho}_{-0}+\left(O(1) e^{-\theta t} \frac{1}{|k|}-O(1) e^{-\theta t}\right) \tilde{k} \tilde{k} \cdot \hat{u}_{-0} \\
& +O(1) e^{-\theta t}\left[\left(-\tilde{k} \times\left(\tilde{k} \times \hat{u}_{-0}\right)\right)-\left(-\tilde{k} \times\left(\tilde{k} \times \hat{u}_{+, 0}\right)\right)\right] \\
& +\frac{O(1)}{|k|} e^{-\theta k^{-2} t}\left(-\tilde{k} \times\left(\tilde{k} \times \hat{E}_{0}\right)\right)+\left(\frac{O(1)}{|k|^{2}} e^{-\theta t}-\frac{O(1)}{|k|^{2}} e^{-\theta k^{-2} t}\right)\left(-\tilde{k} \times\left(\tilde{k} \times \hat{B}_{0}\right)\right) .
\end{aligned}
$$

Furthermore, we have

$$
\hat{E}=-\tilde{k} \times(\tilde{k} \times \hat{E})-\frac{i \tilde{k}}{|k|}\left(\hat{\rho}_{+}-\hat{\rho}_{-}\right) .
$$

Therefore, multiplying the first and the third equation of (3.29) by $\tilde{k}$ and substituting into the above equation, we obtain

$$
\begin{aligned}
\hat{E}= & -\left[\left(O(1)-O(1)|k|^{-1}\right) e^{-\theta t} \hat{\rho}_{+0}-\left(O(1)|k|^{-1}-O(1)\right) e^{-\theta t} i k \cdot \hat{u}_{+0}\right. \\
& \left.+\left(O(1)|k|^{-1}-O(1)\right) e^{-\theta t} \hat{\rho}_{-0}-\left(O(1)+O(1)|k|^{-1}\right) e^{-\theta t} i k \cdot \hat{u}_{-0}\right] \\
& +\left(-O(1) e^{-\theta t}+O(1) e^{-\theta k^{-2} t}\right)\left[\left(-\tilde{k} \times\left(\tilde{k} \times \hat{u}_{-0}\right)\right)-\left(-\tilde{k} \times\left(\tilde{k} \times \hat{u}_{+, 0}\right)\right)\right] \\
& +O(1) e^{-\theta k^{-2} t}\left(-\tilde{k} \times\left(\tilde{k} \times \hat{E}_{0}\right)\right)+\frac{O(1)}{|k|} e^{-\theta k^{-2} t}\left(-\tilde{k} \times\left(\tilde{k} \times \hat{B}_{0}\right)\right) .
\end{aligned}
$$

Since $\tilde{k} \tilde{k} \cdot \hat{B}=0$, we have

$$
\begin{aligned}
\hat{B}= & O(1) i|k| e^{-\theta k^{-2} t}\left(-\tilde{k} \times\left(\tilde{k} \times \hat{u}_{-, 0}\right)\right)-\left(-\tilde{k} \times\left(\tilde{k} \times \hat{u}_{+, 0}\right)\right. \\
& -O(1) i|k| e^{-\theta t}\left(-\tilde{k} \times\left(\tilde{k} \times \hat{E}_{0}\right)\right)+O(1) e^{-\theta k^{-2} t}\left(-\tilde{k} \times\left(\tilde{k} \times \hat{B}_{0}\right)\right) .
\end{aligned}
$$

Subsituting the first equation of (3.59) into the equation (3.54) results in

$$
\begin{aligned}
\left(-\tilde{k} \times\left(\tilde{k} \times \hat{u}_{+}\right)\right)+ & \left(-\tilde{k} \times\left(\tilde{k} \times \hat{u}_{-}\right)\right)=e^{-\theta t}\left[\left(-\tilde{k} \times\left(\tilde{k} \times \hat{u}_{+0}\right)\right)+\left(-\tilde{k} \times\left(\tilde{k} \times \hat{u}_{-0}\right)\right)\right] \\
& +\int_{0}^{t} e^{-(t-s)}\left[O(1) e^{-\theta s}\left(-\tilde{k} \times\left(\tilde{k} \times \hat{u}_{-0}\right)\right)-\left(-\tilde{k} \times\left(\tilde{k} \times \hat{u}_{+0}\right)\right)\right. \\
& +\frac{O(1)}{|k|} e^{-\theta k^{-2} s}\left(-\tilde{k} \times\left(\tilde{k} \times \hat{E}_{0}\right)\right) \\
& \left.+\left(\frac{O(1)}{|k|^{2}} e^{-\theta s}-\frac{O(1)}{|k|^{2}} e^{-\theta k^{-2} s}\right)\left(-\tilde{k} \times\left(\tilde{k} \times \hat{B}_{0}\right)\right)\right] d s .
\end{aligned}
$$


Next, substuting equation (3.30) into the equation (3.62), we obtain

$$
\begin{aligned}
\hat{u}_{+}(t, k) & +\hat{u}_{-}(t, k)=\left(O(1) e^{-\theta t} \tilde{k} \tilde{k}-O(1) e^{-\theta t} \frac{i \tilde{k}}{|k|} i\right) \hat{\rho}_{+0}+\left(O(1) e^{-\theta t}-O(1) e^{-\theta t} \frac{1}{|k|}\right) \tilde{k} \tilde{k} \cdot \hat{u}_{+0} \\
& +\left(O(1) e^{-\theta t} \frac{i \tilde{k}}{|k|}-O(1) e^{-\theta t} i \tilde{k} \tilde{k}\right) \hat{\rho}_{-0}+\left(O(1) e^{-\theta t} \frac{1}{|k|}-O(1) e^{-\theta t}\right) \tilde{k} \tilde{k} \cdot \hat{u}_{-0} \\
& +e^{-\theta t}\left[\left(-\tilde{k} \times\left(\tilde{k} \times \hat{u}_{+0}\right)\right)+\left(-\tilde{k} \times\left(\tilde{k} \times \hat{u}_{-0}\right)\right)\right] \\
& +O(1)\left(e^{-\theta t}-e^{-t}\right)\left[\left(-\tilde{k} \times\left(\tilde{k} \times \hat{u}_{+0}\right)\right)+\left(-\tilde{k} \times\left(\tilde{k} \times \hat{u}_{-0}\right)\right)\right] \\
& +O(1)\left(e^{-\theta k^{-2} t}-e^{-t}\right)\left(-\tilde{k} \times\left(\tilde{k} \times \hat{E}_{0}\right)\right) \\
& +O(1)\left(e^{-\theta t}-e^{-t}\right)\left(-\tilde{k} \times\left(\tilde{k} \times \hat{B}_{0}\right)\right)+O(1)\left(e^{-\theta k^{-2} t}-e^{-t}\right)\left(-\tilde{k} \times\left(\tilde{k} \times \hat{B}_{0}\right)\right)
\end{aligned}
$$

Now, taking the sum and difference respctively of the equations (3.60) and (3.63), we obtain

$$
\begin{aligned}
\hat{u}_{ \pm}(t, k) & =\left(O(1) e^{-\theta t} \tilde{k} \tilde{k}-O(1) e^{-\theta t} \frac{i \tilde{k}}{|k|} i\right) \hat{\rho}_{+0}+\left(O(1) e^{-\theta t}-O(1) e^{-\theta t} \frac{1}{|k|}\right) \tilde{k} \tilde{k} \cdot \hat{u}_{+0} \\
& +\left(O(1) e^{-\theta t} \frac{i \tilde{k}}{|k|}-O(1) e^{-\theta t} i \tilde{k} \tilde{k}\right) \hat{\rho}_{-0}+\left(O(1) e^{-\theta t} \frac{1}{|k|}-O(1) e^{-\theta t}\right) \tilde{k} \tilde{k} \cdot \hat{u}-0 \\
& +O(1) e^{-\theta t}\left[\left(-\tilde{k} \times\left(\tilde{k} \times \hat{u}_{+0}\right)\right)+\left(-\tilde{k} \times\left(\tilde{k} \times \hat{u}_{-0}\right)\right)\right] \\
& +\frac{O(1)}{|k|} e^{-\theta k^{-2} t}\left(-\tilde{k} \times\left(\tilde{k} \times \hat{E}_{0}\right)\right)+\left(\frac{O(1)}{|k|^{2}} e^{-\theta t}-\frac{O(1)}{|k|^{2}} e^{-\theta k^{-2} t}\right)\left(-\tilde{k} \times\left(\tilde{k} \times \hat{B}_{0}\right)\right) \\
& +\frac{i \tilde{k}}{|k|^{2}} O(1) e^{-\theta t} \hat{\rho}_{+0}+\frac{O(1)}{|k|} e^{-\theta t} \tilde{k} \tilde{k} \cdot \hat{u}_{+0}-i \tilde{k} O(1) e^{-\theta t} \hat{\rho}_{-0} \\
& +O(1) e^{-\theta t} \tilde{k} \tilde{k} \cdot \hat{u}_{-0}+e^{-\theta t}\left[\left(-\tilde{k} \times\left(\tilde{k} \times \hat{u}_{+0}\right)\right)+\left(-\tilde{k} \times\left(\tilde{k} \times \hat{u}_{-0}\right)\right)\right] \\
& +O(1) e^{-\theta t}+O(1) e^{-\theta k^{-2} t}+O(1) e^{-\theta t}-O(1) e^{-\theta k^{-2} t}-O(1) e^{-t} .
\end{aligned}
$$

\subsubsection{Combine the Parallel Part and Perpendicular Part}

We will decompose the solution $U=\left[\rho_{ \pm}, u_{ \pm}, E, B\right]$ into the parallel part and perpendicular part.

Theorem 3.3. Let $U=\left[\rho_{ \pm}, u_{ \pm}, E, B\right]$ be the solution to the Cauchy problem (3.1) with intial data (3.2) on the linearized homogeneous system with initial data $U_{0}=$ $\left[\rho_{ \pm, 0}, u_{ \pm, 0}, E_{0}, B_{0}\right]$ satisfying the condition (3.3). For all $t \geq 0$ and all $k \in \mathbb{R}^{3}$ satisfy- 
ing $|k| \neq 0$, we may decompose $U$ via

$$
\left[\begin{array}{c}
\hat{\rho}_{+}(t, k) \\
\hat{\rho}_{-}(t, k) \\
\hat{u}_{+}(t, k) \\
\hat{u}_{-}(t, k) \\
\hat{E}(t, k) \\
\hat{B}(t, k)
\end{array}\right]=\left[\begin{array}{c}
\hat{\rho}_{+}(t, k) \\
\hat{\rho}_{-}(t, k) \\
\hat{u}_{+, \|}(t, k) \\
\hat{u}_{-, \|}(t, k) \\
\hat{E}_{\|}(t, k) \\
0
\end{array}\right]+\left[\begin{array}{c}
0 \\
0 \\
\hat{u}_{+, \perp}(t, k) \\
\hat{u}_{-, \perp}(t, k) \\
\hat{E}_{\perp}(t, k) \\
\hat{B}_{\perp}(t, k)
\end{array}\right]
$$

where $\hat{u}_{ \pm, \|}, \hat{u}_{ \pm, \perp}$ are defined by

$$
\hat{u}_{ \pm, \|}=\tilde{k} \tilde{k} \cdot \hat{u}_{ \pm}, \quad \hat{u}_{ \pm, \perp}=-\tilde{k} \times\left(\tilde{k} \times \hat{u}_{ \pm}\right)=\left(\mathbf{I}_{3}-\tilde{k} \otimes \tilde{k}\right) \hat{u}_{ \pm} .
$$

simillarly, for $\hat{E}_{\|}(t, k), \hat{E}_{\perp}(t, k)$ and $\hat{B}_{\perp}(t, k)$

$$
\left[\begin{array}{l}
M_{5}(t, k) \\
M_{3}(t, k) \\
M_{4}(t, k)
\end{array}\right]:=\left[\begin{array}{c}
\hat{u}_{ \pm, \perp}(t, k) \\
\hat{E}_{\perp}(t, k) \\
\hat{B}_{\perp}(t, k)
\end{array}\right],\left[\begin{array}{l}
M_{5,0}(t, k) \\
M_{3,0}(t, k) \\
M_{4,0}(t, k)
\end{array}\right]:=\left[\begin{array}{c}
\hat{u}_{ \pm 0, \perp}(t, k) \\
\hat{E}_{0, \perp}(t, k) \\
\hat{B}_{0, \perp}(t, k)
\end{array}\right]
$$

Then we have the matrices $G_{11 \times 11}^{I}(t, k)$ and $G_{9 \times 9}^{I I}(t, k)$ such that

$$
\left[\begin{array}{c}
\hat{\rho}_{+}(t, k) \\
\hat{\rho}_{-}(t, k) \\
\hat{u}_{+, \|}(t, k) \\
\hat{u}_{-, \|}(t, k) \\
\hat{E}_{\|}(t, k)
\end{array}\right]=G_{11 \times 11}^{I}(t, k)\left[\begin{array}{c}
\hat{\rho}_{+0}(t, k) \\
\hat{\rho}_{-0}(t, k) \\
\hat{u}_{+0, \|}(t, k) \\
\hat{u}_{-0, \|}(t, k) \\
\hat{E}_{0 \|}(t, k)
\end{array}\right]
$$

and

$$
\left[\begin{array}{c}
M_{5}(t, k) \\
M_{3}(t, k) \\
M_{4}(t, k)
\end{array}\right]=G_{9 \times 9}^{I I}(t, k)\left[\begin{array}{l}
M_{5,0}(t, k) \\
M_{3,0}(t, k) \\
M_{4,0}(t, k)
\end{array}\right]
$$

\subsection{Refined $L^{p}-L^{q}$ time-decay property}

In this subsection, we seek to refine the $L^{p}-L^{q}$ time-decay property for every component of the solution $U=\left[\rho_{ \pm}, u_{ \pm}, E, B\right]$. To do so, we use Theorem 3.3 and find the delicate time-frequency pointwise estimates on $\hat{U}=\left[\hat{\rho}_{ \pm}, \hat{u}_{ \pm}, \hat{E}, \hat{B}\right]$ as follows.

Theorem 3.4. Let $1 \leq p, r \leq 2 \leq q \leq \infty, l \geq 0$ and let $m \geq 0$ be an integer. Define $\left[l+3\left(\frac{1}{r}-\frac{1}{q}\right)\right]_{+}=\left\{\begin{array}{l}{\left[l+3\left(\frac{1}{r}-\frac{1}{q}\right)\right]_{-}+1, \quad \text { when } r \neq 2 \text { or } q \neq 2 \text { or } l \text { is not an integer }} \\ l, \quad \text { when } r=q=2 \text { and } l \text { is an integer }\end{array}\right.$ 
where $[\cdot]_{-}$denotes the integer part of the argument. Suppose $U_{0}$ satisfies (3.3). Then for any $t \geq 0, \nabla^{m} e^{t L} U_{0}$ satisfies the following time-decay property:

$$
\left\|\nabla^{m} e^{t L} U_{0}\right\|_{L^{q}} \leq C(1+t)^{-\frac{3}{2}\left(\frac{1}{p}-\frac{1}{q}\right)-\frac{m}{2}}\left\|U_{0}\right\|_{L^{p}}+C(1+t)^{-\frac{l}{2}}\left\|\nabla^{m+\left[l+3\left(\frac{1}{r}-\frac{1}{q}\right)\right]_{+}} U_{0}\right\|_{L^{r}}
$$

where $C=C(p, q, r, l, m)$.

Lemma 3.5. Suppose $U=\left[\rho_{ \pm}, u_{ \pm}, E, B\right]$ is the solution to the linearized homogeneous system (3.1) with initial data $U_{0}=\left[\rho_{0, \pm}, u_{0, \pm}, E_{0}, B_{0}\right]$ satisfying (3.3). Then there exist constants $\theta>0, C>0$ such that for all $t \geq 0, k \in \mathbb{R}^{3}$,

$$
\begin{aligned}
& \left|\hat{\rho_{ \pm}}(t, k)\right| \leq C\left|\left[\hat{\rho}_{ \pm 0}(k), \hat{u}_{ \pm 0}(k)\right]\right| \cdot \begin{cases}\left(e^{-\theta t}+e^{-\theta|k|^{2} t}\right) & \text { if }|k| \leq 1 \\
e^{-\theta t} & \text { if }|k| \geq 1\end{cases} \\
& \left|\hat{u_{ \pm}}(t, k)\right| \leq C e^{-\frac{t}{2}}\left|\left[\hat{\rho}_{ \pm 0}(k), \hat{u}_{ \pm 0}(k), \hat{E}_{0}(k)\right]\right| \\
& +C\left|\left[\hat{\rho}_{ \pm 0}(k), \hat{u}_{ \pm 0}(k), \hat{E}_{0}(k), \hat{B}_{0}(k)\right]\right| \cdot \begin{cases}\left(e^{-\theta t}+|k| e^{-\theta|k|^{2} t}\right) & \text { if }|k| \leq 1 \\
\left(e^{-\theta t}+\frac{1}{|k|} e^{-\frac{\theta t}{|k|^{2}}}\right) & \text { if }|k| \geq 1\end{cases} \\
& |\hat{E}(t, k)| \leq C\left|\left[\hat{\rho}_{ \pm 0}(k), \hat{u}_{ \pm 0}(k), \hat{E}_{0}(k), \hat{B}_{0}(k)\right]\right| \cdot \begin{cases}e^{-\theta t} & \text { if }|k| \leq 1 \\
\left(\frac{1}{|k|} e^{-\theta t}+e^{-\frac{\theta t}{|k|^{2}}}\right) & \text { if }|k| \geq 1\end{cases} \\
& +C\left|\left[\hat{u}_{ \pm 0}(k), \hat{E}_{0}(k), \hat{B}_{0}(k)\right]\right| \cdot \begin{cases}\left(e^{-\theta t}+|k| e^{-\theta|k|^{2} t}\right) & \text { if }|k| \leq 1 \\
\left(\frac{1}{|k|^{2}} e^{-\theta t}+e^{-\frac{\theta t}{|k|^{2}}}\right) & \text { if }|k| \geq 1\end{cases} \\
& |\hat{B}(t, k)| \leq C\left|\left[\hat{u}_{ \pm 0}(k), \hat{E}_{0}(k), \hat{B}_{0}(k)\right]\right| \cdot \begin{cases}\left(|k| e^{-\theta t}+e^{-\theta|k|^{2} t}\right) & \text { if }|k| \leq 1 \\
\left(\frac{1}{|k|} e^{-\theta t}+e^{-\frac{\theta t}{|k|^{2}}}\right) & \text { if }|k| \geq 1\end{cases}
\end{aligned}
$$

Proof: The upper bound (3.69) follows from (3.65) and the first and third equations of (3.25) and (3.29). The first term of the right-hand side of (3.70) is obtained from (3.57) and (3.64), while the right-hand side of (3.72) is obtained from (3.53), (3.61). The remaining terms on the right-hand sides of (3.70) and (3.72) were found by considering the upper bound of $\left[M_{5}, M_{3}, M_{4}\right]$. Equivalently, we could consider $\left[0, \hat{u}_{ \pm \perp}, \hat{E}_{\perp}, \hat{B_{\perp}}\right]$ in terms of $\left[\hat{u}_{ \pm, 0}, \hat{E}_{0}, \hat{B}_{0}\right]$ by (3.65). Applying Lemma 3.2 to (3.43), we find

$$
\left[\begin{array}{l}
c_{1} \\
c_{2} \\
c_{3}
\end{array}\right]=\left[\begin{array}{ccc}
O(1)|k|^{2} \mathbf{I}_{3} & -O(1)|k|^{2} \mathbf{I}_{3} & O(1)|k| i \tilde{k} \times \\
-O(1)|k|^{2} \mathbf{I}_{3} & O(1) \mathbf{I}_{3} & -O(1)|k| i \tilde{k} \times \\
-O(1) \mathbf{I}_{3} & O(1) \mathbf{I}_{3} & O(1)|k| i \tilde{k} \times
\end{array}\right]\left[\begin{array}{c}
M_{5,0} \\
M_{3,0} \\
M_{4,0}
\end{array}\right]
$$


when $|k| \rightarrow 0$ and

$$
\left[\begin{array}{l}
c_{1} \\
c_{2} \\
c_{3}
\end{array}\right]=\left[\begin{array}{ccc}
O(1)|k|^{-2} \mathbf{I}_{3} & -O(1)|k|^{-4} \mathbf{I}_{3} & O(1)|k|^{-3} i \tilde{k} \times \\
-O(1)|k|^{-2} \mathbf{I}_{3} & O(1) \mathbf{I}_{3} & -O(1)|k|^{-3} i \tilde{k} \times \\
-O(1)|k|^{-1} \mathbf{I}_{3} & O(1)|k|^{-3} \mathbf{I}_{3} & O(1) i \tilde{k} \times
\end{array}\right]\left[\begin{array}{c}
M_{5,0} \\
M_{3,0} \\
M_{4,0}
\end{array}\right]
$$

when $|k| \rightarrow \infty$. Furthermore, it follows that

$$
\frac{2\left(1+\alpha_{+}+\alpha_{-}+\beta\right)}{\left(1+\alpha_{+}+\alpha_{-}+\beta\right)^{2}+\omega^{2}}= \begin{cases}O(1) & \text { if }|k| \rightarrow 0 \\ O(1)|k|^{-2} & \text { if }|k| \rightarrow \infty\end{cases}
$$

and

$$
\frac{2 \omega}{\left(1+\alpha_{+}+\alpha_{-}+\beta\right)^{2}+\omega^{2}}= \begin{cases}O(1) & \text { if }|k| \rightarrow 0 \\ O(1)|k|^{-1} & \text { if }|k| \rightarrow \infty .\end{cases}
$$

Substituting the equations (3.73) and (3.74) into equation (3.46), we obtain

$$
\begin{aligned}
M_{5}(t, k)=2( & \left.(1)|k|^{2} M_{5,0}-O(1)|k|^{2} M_{3,0}+O(1)|k| i \tilde{k} \times M_{4,0}\right) \cdot O(1) e^{\eta(k) t} \\
+ & 2\left(-O(1)|k|^{2} M_{5,0}+O(1) M_{3,0}-O(1)|k| i \tilde{k} \times M_{4,0}\right) \\
\cdot & (O(1) \cos \omega t+O(1) \sin \omega t) e^{\beta(k) t} \\
+ & 2\left(-O(1) M_{5,0}+O(1) M_{3,0}+O(1)|k| i \tilde{k} \times M_{4,0}\right) \\
\cdot & (O(1) \sin \omega t-O(1) \cos \omega t) e^{\beta(k) t}
\end{aligned}
$$

when $|k| \rightarrow 0$. When $|k| \rightarrow \infty$,

$$
\begin{aligned}
M_{5}(t, k)=2( & \left.O(1)|k|^{-2} M_{5,0}-O(1)|k|^{-4} M_{3,0}+O(1)|k|^{-3} i \tilde{k} \times M_{4,0}\right) \cdot O(1)|k|^{2} e^{\eta(k) t} \\
+ & 2\left(-O(1)|k|^{-2} M_{5,0}+O(1) M_{3,0}-O(1)|k|^{-3} i \tilde{k} \times M_{4,0}\right) \\
& \cdot\left(O(1)|k|^{-2} \cos \omega t+O(1)|k|^{-1} \sin \omega t\right) e^{\beta(k) t} \\
& +2\left(-O(1)|k|^{-1} M_{5,0}+O(1)|k|^{-3} M_{3,0}+O(1) i \tilde{k} \times M_{4,0}\right) \\
& \cdot\left(O(1)|k|^{-2} \sin \omega t-O(1)|k|^{-1} \cos \omega t\right) e^{\beta(k) t} .
\end{aligned}
$$

According to Lemma 3.2, there is $\theta>0$ such that

$$
\begin{cases}\sigma(k) \geq-\theta|k|^{2}, & \beta(k)=-\frac{\sigma(k)+1}{2} \geq-\theta \quad \text { if }|k| \leq 1 \\ \sigma(k) \geq-\theta, & \beta(k)=-\frac{\sigma(k)+1}{2} \geq-\frac{\theta t}{|k|^{2}}\end{cases}
$$

Therefore, since $\left|\left[M_{5,0}, M_{3,0}, M_{4,0}\right]\right| \leq\left|\left[\hat{u}_{ \pm, 0}(k), \hat{E}_{0}(k), \hat{B}_{0}(k)\right]\right|$, we obtain

$$
\left|M_{5}(t, k)\right| \leq C\left|\left[\hat{u}_{0}(k), \hat{E}_{0}(k), \hat{B}_{0}(k)\right]\right| \cdot \begin{cases}\left(e^{-\theta t}+|k| e^{-\theta|k|^{2} t}\right) & \text { if }|k| \leq 1 \\ \left(e^{-\theta t}+\frac{1}{|k|} e^{-\frac{\theta t}{|k|^{2}}}\right) & \text { if }|k| \geq 1\end{cases}
$$


This corresponds to the second term on the right-hand side of equation (3.70), and is therefore the upper bounded of $\hat{u}_{ \pm \perp}(t, k)$.

Now, to check equations (3.71) and (3.72), we need to use

$$
\frac{\beta}{\beta^{2}+\omega^{2}}= \begin{cases}-O(1) & \text { if }|k| \rightarrow 0 \\ O(1)|k|^{-4} & \text { if }|k| \rightarrow \infty\end{cases}
$$

and

$$
\frac{\beta}{\beta^{2}+\omega^{2}}= \begin{cases}O(1) & \text { if }|k| \rightarrow 0 \\ O(1)|k|^{-1} & \text { if }|k| \rightarrow \infty\end{cases}
$$

Similarly, to estimate $M_{4}(t, k)$, we substitute the above computation into equation (3.47) to obtain

$$
\begin{aligned}
M_{4}(t, k)=- & i k \\
& \times\left(O(1)|k|^{2} M_{5,0}-O(1)|k|^{2} M_{3,0}+O(1)|k| i \tilde{k} \times M_{4,0}\right) \cdot-O(1)|k|^{-2} e^{\eta(k) t} \\
& \cdot(-O(1) \cos \omega t+O(1) \sin \omega t) e^{\beta(k) t} \\
& -i k \times\left(-O(1) M_{5,0}+O(1) M_{3,0}+O(1)|k| \tilde{k} \times M_{4,0}\right) \\
& \cdot(-O(1) \sin \omega t-O(1) \cos \omega t) e^{\beta(k) t}
\end{aligned}
$$

when $|k| \rightarrow 0$. When $|k| \rightarrow \infty$,

$$
\begin{aligned}
M_{4}(t, k) & =-i k \times\left(-O(1)|k|^{-2} M_{5,0}-O(1)|k|^{-4} M_{3,0}+O(1)|k|^{-3} i \tilde{k} \times M_{4,0}\right) \cdot-O(1) e^{\eta(k) t} \\
& -i k \times\left(-O(1)|k|^{-2} M_{5,0}+O(1) M_{3,0}-O(1)|k|^{-3} i \tilde{k} \times M_{4,0}\right) \\
& \cdot\left(-O(1)|k|^{-4} \cos \omega t+O(1)|k|^{-1} \sin \omega t\right) e^{\beta(k) t} \\
& -i k \times\left(-O(1)|k|^{-1} M_{5,0}+O(1)|k|^{-3} M_{3,0}+O(1) i \tilde{k} \times M_{4,0}\right) \\
& \cdot\left(-O(1)|k|^{-4} \sin \omega t-O(1)|k|^{-1} \cos \omega t\right) e^{\beta(k) t}
\end{aligned}
$$

Therefore, for $|k| \leq 1$ and $|k| \geq 1$ it follows that

$$
\left|M_{4}(t, k)\right| \leq C\left|\left[\hat{u}_{0}(k), \hat{E}_{0}(k), \hat{B}_{0}(k)\right]\right| \cdot \begin{cases}\left(e^{-\theta t}+|k| e^{-\theta|k|^{2} t}\right) & \text { if }|k| \leq 1 \\ \left(\frac{1}{|k|^{2}} e^{-\theta t}+e^{-\frac{\theta t}{|k|^{2}}}\right) & \text { if }|k| \geq 1\end{cases}
$$

Moreover, using equation (3.41), we get

$$
\begin{aligned}
M_{3}(t, k)=( & \left.O(1)|k|^{2} M_{5,0}-O(1)|k|^{2} M_{3,0}+O(1)|k| i \tilde{k} \times M_{4,0}\right) \cdot e^{\eta(k) t} \\
& +\left(-O(1)|k|^{2} M_{5,0}+O(1) M_{3,0}-O(1)|k| i \tilde{k} \times M_{4,0}\right) \cdot \cos \omega t e^{\beta(k) t} \\
& +\left(-O(1) M_{5,0}+O(1) M_{3,0}+O(1)|k| i \tilde{k} \times M_{4,0}\right) \cdot \sin \omega t e^{\beta(k) t}
\end{aligned}
$$


when $|k| \rightarrow 0$. When $|k| \rightarrow \infty$,

$$
\begin{aligned}
M_{3}(t, k)=(- & \left.O(1)|k|^{-2} M_{5,0}-O(1)|k|^{-4} M_{3,0}+O(1)|k|^{-3} i \tilde{k} \times M_{4,0}\right) \cdot e^{\eta(k) t} \\
& +\left(-O(1)|k|^{-2} M_{5,0}+O(1) M_{3,0}-O(1)|k|^{-3} i \tilde{k} \times M_{4,0}\right) \cdot \cos \omega t e^{\beta(k) t} \\
& +\left(-O(1)|k|^{-1} M_{5,0}+O(1)|k|^{-3} M_{3,0}+O(1) i \tilde{k} \times M_{4,0}\right) \cdot \sin \omega t .
\end{aligned}
$$

Hence, for $|k| \leq 1$ and $|k| \geq 1$, (3.72) holds and this concludes of the proof.

Theorem 3.6. Let $1 \leq p, r \leq 2 \leq q \leq \infty, l \geq 0$ and let $m \geq 0$ be an integer. Assume $U(t)=e^{t L} U_{0}$ is the solution of the initial value problem (3.1), (3.2) with initial data $\left[\rho_{ \pm 0}, u_{ \pm 0}, E_{0}, B_{0}\right]$ which satisfies (3.3). Then for any $t \geq 0, U=\left[\rho_{ \pm}, u_{ \pm}, E, B\right]$ satisfies

$$
\begin{gathered}
\left\|\nabla^{m} \rho_{ \pm}(t)\right\|_{L^{q}} \leq C(1+t)^{-\frac{3}{2}\left(\frac{1}{p}-\frac{1}{q}\right)-\frac{m}{2}}\left\|\left[\hat{\rho}_{ \pm 0}, \hat{u}_{ \pm, 0}\right]\right\|_{L^{p}}+C\left\|\left[\rho_{ \pm 0}, u_{ \pm 0}\right]\right\|_{L^{r}} \\
\left\|\nabla^{m} u_{ \pm}(t)\right\|_{L^{q}} \leq C e^{-\frac{t}{2}}\left(\left\|\rho_{ \pm 0}\right\|_{L^{p}}+\left\|\nabla^{m+\left[3\left(\frac{1}{r}-\frac{1}{q}\right)\right]_{+}}\left[\rho_{ \pm 0}, u_{ \pm 0}\right]\right\|_{L^{r}}\right) \\
+C(1+t)^{-\frac{3}{2}\left(\frac{1}{p}-\frac{1}{q}\right)-\frac{m+1}{2}}\left\|\left[u_{ \pm 0}, E_{0}, B_{0}\right]\right\|_{L^{p}} \\
+C(1+t)^{-\frac{l+1}{2}}\left\|\nabla^{m+\left[l+3\left(\frac{1}{r}-\frac{1}{q}\right)\right]_{+}}\left[u_{ \pm 0}, E_{0}, B_{0}\right]\right\|_{L^{r}} \\
\left\|\nabla^{m} E(t)\right\|_{L^{q}} \leq C(1+t)^{-\frac{3}{2}\left(\frac{1}{p}-\frac{1}{q}\right)-\frac{m+1}{2}}\left\|\left[u_{ \pm 0}, E_{0}, B_{0}\right]\right\|_{L^{p}} \\
+C(1+t)^{-\frac{l}{2}}\left\|\nabla^{m+\left[l+3\left(\frac{1}{r}-\frac{1}{q}\right)\right]_{+}}\left[u_{ \pm 0}, E_{0}, B_{0}\right]\right\|_{L^{r}} \\
\left\|\nabla^{m} B(t)\right\|_{L^{q}} \leq C(1+t)^{-\frac{3}{2}\left(\frac{1}{p}-\frac{1}{q}\right)-\frac{m}{2}}\left\|\left[u_{ \pm 0}, E_{0}, B_{0}\right]\right\|_{L^{p}} \\
+C(1+t)^{-\frac{l}{2}}\left\|\nabla^{m+\left[l+3\left(\frac{1}{r}-\frac{1}{q}\right)\right]_{+}}\left[u_{ \pm 0}, E_{0}, B_{0}\right]\right\|_{L^{r}}
\end{gathered}
$$

where $C=C(p, q, r, l, m)$ and $\left[l+3\left(\frac{1}{r}-\frac{1}{q}\right)\right]_{+}$is defined in (3.67).

Proof : Let $1 \leq p, r \leq 2 \leq q \leq \infty$ and $m$ be an non-negative integer. Let $q^{\prime}$ satsify $\frac{1}{q^{\prime}}+\frac{1}{q}=1$. Using the Hausdorff-Young inequality and (3.69) we prove (3.77) as follows,

$$
\left\|\nabla^{m} \rho_{ \pm}(t, k)\right\|_{L_{x}^{q}} \leq C\left\|\left.|| k\right|^{m} \hat{\rho}_{ \pm}\right\|_{L^{q^{\prime}(|k| \leq 1)}}+C\left\||k|^{m} \hat{\rho}_{ \pm}\right\|_{L^{q^{\prime}}(|k| \geq 1)}
$$

We estimate of the first term of (3.81) using Hölder inequality $\frac{1}{q^{\prime}}=\frac{1}{p^{\prime}}+\frac{p^{\prime}-q^{\prime}}{p^{\prime} q^{\prime}}$ with 
$\frac{1}{p^{\prime}}+\frac{1}{p}=1$ and fixing $\varepsilon>0$ sufficiently small; that is,

$$
\begin{aligned}
&\left\||k|^{m}\left[\hat{\rho}_{ \pm 0}, \hat{u}_{ \pm 0}\right]\left[e^{-\theta t}+e^{-\theta|k|^{2} t}\right]\right\|_{L^{q^{\prime}}(|k| \leq 1)} \\
& \quad=\left\||k|^{-\frac{p^{\prime}-q^{\prime}}{p^{\prime} q^{\prime}}(3-\varepsilon)}|k|^{m+\frac{p^{\prime}-q^{\prime}}{p^{\prime} q^{\prime}}(3-\varepsilon)}\left[\hat{\rho}_{ \pm 0}, \hat{u}_{ \pm 0}\right]\left[e^{-\theta t}+e^{-\theta|k|^{2} t}\right]\right\|_{L^{q^{\prime}}(|k| \leq 1)} \\
& \quad \leq\left\||k|^{-(3-\varepsilon)}\right\|^{-\frac{p^{\prime}-q^{\prime}}{p^{\prime} q^{\prime}}}\left\||k|^{m+\frac{p^{\prime}-q^{\prime}}{p^{\prime} q^{\prime}}}\left[\hat{\rho}_{ \pm 0}, \hat{u}_{ \pm 0}\right]\left[e^{-\theta t}+e^{-\theta|k|^{2} t}\right]\right\|_{L^{p^{\prime}(|k| \leq 1)}} \\
& \quad \leq\left\||k|^{m+\frac{p^{\prime}-q^{\prime}}{p^{\prime} q^{\prime}}(3-\varepsilon)}\left[\hat{\rho}_{ \pm 0}, \hat{u}_{ \pm 0}\right]\left[e^{-\theta t}+e^{-\theta|k|^{2} t}\right]\right\|_{L^{p^{\prime}}(|k| \leq 1)} \\
& \quad \leq C e^{-\theta t}\left\|\left[\hat{\rho}_{ \pm 0}, \hat{u}_{ \pm 0}\right]\right\|_{L^{p^{\prime}}(|k| \leq 1)}+C(t+1)^{-\frac{3}{2}\left(\frac{1}{q^{\prime}}-\frac{1}{p^{\prime}}\right)-\frac{m+1}{2}}\left[\hat{\rho}_{ \pm 0}, \hat{u}_{ \pm 0}\right] \|_{L^{p^{\prime}}(|k| \leq 1)} \\
& \leq C e^{-\theta t}\left\|\left[\rho_{ \pm 0}, u_{ \pm 0}\right]\right\|_{L^{p}(|k| \leq 1)}+C(t+1)^{-\frac{3}{2}\left(\frac{1}{p}-\frac{1}{q}\right)-\frac{m+1}{2}}\left\|\left[\rho_{ \pm 0}, u_{ \pm 0}\right]\right\|_{L^{p}(|k| \leq 1)} .
\end{aligned}
$$

Now we estimate of the second term; by taking $\varepsilon>0$ sufficiently small and using Hölder inequality $\frac{1}{q^{\prime}}=\frac{1}{r^{\prime}}+\frac{r^{\prime}-q^{\prime}}{r^{\prime} q^{\prime}}$ with $\frac{1}{r^{\prime}}+\frac{1}{r}=1$ we obtain

$$
\begin{aligned}
\left\||k|^{m}\left[\hat{\rho}_{ \pm 0}, \hat{u}_{ \pm 0}\right]\left[e^{-\theta t}+e^{-\theta|k|^{2} t}\right]\right\|_{L^{q^{\prime}}(|k| \geq 1)} \\
\quad=\left\||k|^{-\frac{r^{\prime}-q^{\prime}}{r^{\prime} q^{\prime}}(3+\varepsilon)}|k|^{m+\frac{p^{\prime}-q^{\prime}}{p^{\prime} q^{\prime}}(3+\varepsilon)}\left[\hat{\rho}_{ \pm 0}, \hat{u}_{ \pm 0}\right] e^{-\theta t}\right\|_{L^{q^{\prime}}(|k| \geq 1)} \\
\quad \leq\left\||k|^{-(3+\varepsilon)}\right\|^{-\frac{r^{\prime}-q^{\prime}}{r^{\prime} q^{\prime}}}\left\||k|^{m+\frac{r^{\prime}-q^{\prime}}{r^{\prime} q^{\prime}}(3+\varepsilon)^{\prime}}\left[\hat{\rho}_{ \pm 0}, \hat{u}_{ \pm 0}\right] e^{-\theta t}\right\|_{L^{r^{\prime}}(|k| \geq 1)} \\
\quad \leq C\left\||k|^{m+\left(\frac{1}{q^{\prime}}-\frac{1}{r^{\prime}}\right)(3+\varepsilon)}\left[\hat{\rho}_{ \pm 0}, \hat{u}_{ \pm 0}\right] e^{-\theta t}\right\|_{L^{r^{\prime}(|k| \geq 1)}} \\
\quad \leq C e^{-\theta t}\left\|\nabla^{m+\left[3\left(\frac{1}{r}-\frac{1}{q}\right)\right]_{+}}\left[\rho_{ \pm 0}, u_{ \pm 0}\right]\right\|_{L^{r^{\prime}}(|k| \geq 1)} .
\end{aligned}
$$

We prove (3.78) similarly. That is,

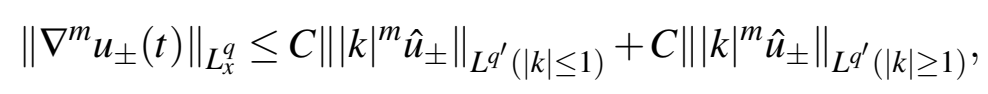

where

$$
\begin{gathered}
\left\||k|^{m} \hat{u}_{ \pm}(t, k)\right\|_{L^{q^{\prime}(|k| \leq 1)}}=\left\||k|^{m} e^{-t / 2}\left[\hat{\rho}_{ \pm 0}, \hat{u}_{ \pm 0}, \hat{E}_{0}\right]\right\|_{L^{q^{\prime}}}+\left\||k|^{m} e^{-\theta t}\left[\hat{\rho}_{ \pm 0}, \hat{u}_{ \pm 0}, \hat{E}_{0}, \hat{B}_{0}\right]\right\|_{L^{q^{\prime}}} \\
+\left\||k|^{m} e^{-\theta|k|^{2} t}\left[\hat{\rho}_{ \pm 0}, \hat{u}_{ \pm 0}, \hat{E}_{0}, \hat{B}_{0}\right]\right\|_{L^{q^{\prime}}}
\end{gathered}
$$

We estimate the first term of (3.82) using the Hölder inequality $\frac{1}{q^{\prime}}=\frac{1}{p^{\prime}}+\frac{p^{\prime}-q^{\prime}}{p^{\prime} q^{\prime}}$ with 
$\frac{1}{p^{\prime}}+\frac{1}{p}=1$ and fixing $\varepsilon>0$ sufficiently small:

$$
\begin{aligned}
\left\||k|^{m} e^{-t / 2}\left[\hat{\rho}_{ \pm 0}, \hat{u}_{ \pm 0}, \hat{E}_{0}\right]\right\|_{L^{q^{\prime}}}=\left(\int_{\mathbb{R}^{3}} \|\left.\left. k\right|^{m} e^{-t / 2}\left[\hat{\rho}_{ \pm 0}, \hat{u}_{ \pm 0}, \hat{E}_{0}\right]\right|^{q^{\prime}}\right)^{1 / q^{\prime}} \\
\quad=\left(\int_{\mathbb{R}^{3}} \|\left.\left. k\right|^{-\frac{p^{\prime}-q^{\prime}}{p^{\prime} q^{\prime}}(3-\varepsilon)}|k|^{m+\frac{p^{\prime}-q^{\prime}}{p^{\prime} q^{\prime}}(3-\varepsilon)} e^{-t / 2}\left[\hat{\rho}_{ \pm 0}, \hat{u}_{ \pm 0}, \hat{E}_{0}\right]\right|^{q^{\prime}}\right)^{1 / q^{\prime}} \\
\quad \leq e^{-t / 2}\left\||k|^{-(3-\varepsilon)}\right\|^{-\frac{p^{\prime}-q^{\prime}}{p^{\prime} q^{\prime}}}\left\||k|^{m+\frac{p^{\prime}-q^{\prime}}{p^{\prime} q^{\prime}}}\left[\hat{\rho}_{ \pm 0}\right]\right\|_{L^{p^{\prime}(|k| \leq 1)}} \\
\leq e^{-t / 2}\left\||k|^{m+\frac{p^{\prime}-q^{\prime}}{p^{\prime} q^{\prime}}(3-\varepsilon)}\left[\hat{\rho}_{ \pm 0}\right]\right\|_{L^{p^{\prime}}(|k| \leq 1)} \leq C e^{-t / 2}\left\|\left[\hat{\rho}_{ \pm 0}\right]\right\|_{L^{p^{\prime}}(|k| \leq 1)} \\
\leq C e^{-t / 2}\left\|\left[\rho_{ \pm 0}\right]\right\|_{L^{p}(|k| \leq 1)} .
\end{aligned}
$$

We estimate the second term of (3.82) by

$$
\begin{aligned}
\left\||k|^{m} e^{-\theta|k|^{2} t}\left[\hat{\rho}_{ \pm 0}, \hat{u}_{ \pm 0}, \hat{E}_{0}, \hat{B}_{0}\right]\right\|_{L^{q^{\prime}}}=\left(\int_{\mathbb{R}^{3}} \|\left.\left. k\right|^{m+1} e^{-\theta|k|^{2} t}\left[\hat{u}_{ \pm 0}, \hat{E}_{0}, \hat{B}_{0}\right]\right|^{q^{\prime}}\right)^{1 / q^{\prime}} \\
\quad=\left(\left.\left.\int_{\mathbb{R}^{3}}|| k\right|^{-\frac{p^{\prime}-q^{\prime}}{p^{\prime} q^{\prime}}(3-\varepsilon)}|k|^{m+\frac{p^{\prime}-q^{\prime}}{p^{\prime} q^{\prime}}(3-\varepsilon)} e^{-\theta|k|^{2} t}\left[\hat{u}_{ \pm 0}, \hat{E}_{0}, \hat{B}_{0}\right]\right|^{q^{\prime}}\right)^{1 / q^{\prime}} \\
\leq\left\||k|^{-(3-\varepsilon)}\right\|^{-\frac{p^{\prime}-q^{\prime}}{p^{\prime} q^{\prime}}}\left\||k|^{m+\frac{p^{\prime}-q^{\prime}}{p^{\prime} q^{\prime}}} e^{-\theta|k|^{2} t}\left[\hat{u}_{ \pm 0}, \hat{E}_{0}, \hat{B}_{0}\right]\right\|_{L^{p^{\prime}}(|k| \leq 1)} \\
\leq e^{-t / 2}\left\||k|^{m+\frac{p^{\prime}-q^{\prime}}{p^{\prime} q^{\prime}}(3-\varepsilon)}\left[\hat{\rho}_{ \pm 0}\right]\right\|_{L^{p^{\prime}}(|k| \leq 1)} \leq C e^{-t / 2}\left\|\left[\hat{\rho}_{ \pm 0}\right]\right\|_{L^{p^{\prime}}(|k| \leq 1)} \\
\leq C e^{-t / 2}\left\|\left[\rho_{ \pm 0}\right]\right\|_{L^{p}(|k| \leq 1)} .
\end{aligned}
$$

We estimate the third term of (3.82) in two parts. First, take $\varepsilon>0$ sufficiently small and use Hölder inequality $\frac{1}{q^{\prime}}=\frac{1}{r^{\prime}}+\frac{r^{\prime}-q^{\prime}}{r^{\prime} q^{\prime}}$ with $\frac{1}{r^{\prime}}+\frac{1}{r}=1$ :

$$
\begin{aligned}
\left\||k|^{m} \hat{u}_{ \pm}(t, k)\right\|_{L^{q^{\prime}}(|k| \geq 1)} & =\left\||k|^{m} e^{-t / 2}\left[\hat{\rho}_{ \pm 0}, \hat{u}_{ \pm 0}, \hat{E}_{0}\right]\right\|_{L^{q^{\prime}}}+\left\||k|^{m} e^{-\theta t}\left[\hat{\rho}_{ \pm 0}, \hat{u}_{ \pm 0}, \hat{E}_{0}, \hat{B}_{0}\right]\right\|_{L^{q^{\prime}}} \\
& +\left\||k|^{m} \frac{1}{|k|} e^{-\theta|k|^{2} t}\left[\hat{\rho}_{ \pm 0}, \hat{u}_{ \pm 0}, \hat{E}_{0}, \hat{B}_{0}\right]\right\|_{L^{q^{\prime}}}
\end{aligned}
$$


where

$$
\begin{aligned}
\left\||k|^{m} e^{-t / 2}\left[\hat{\rho}_{ \pm 0}, \hat{u}_{ \pm 0}, \hat{E}_{0}\right]\right\|_{L^{q^{\prime}}}=\left(\int_{\mathbb{R}^{3}} \|\left.\left. k\right|^{m} e^{-t / 2}\left[\hat{\rho}_{ \pm 0}, \hat{u}_{ \pm 0}, \hat{E}_{0}\right]\right|^{q^{\prime}}\right)^{1 / q^{\prime}} \\
\quad=\left(\left.\left.\int_{\mathbb{R}^{3}}|| k\right|^{-\frac{r^{\prime}-q^{\prime}}{r^{\prime} q^{\prime}}(3+\varepsilon)}|k|^{m+\frac{r^{\prime}-q^{\prime}}{r^{\prime} q^{\prime}}(3+\varepsilon)} e^{-t / 2}\left[\hat{\rho}_{ \pm 0}, \hat{u}_{ \pm 0}, \hat{E}_{0}\right]\right|^{q^{\prime}}\right)^{1 / q^{\prime}} \\
\leq e^{-t / 2}\left\||k|^{-(3+\varepsilon)}\right\|^{-\frac{r^{\prime}-q^{\prime}}{r^{\prime} q^{\prime}}}\left\||k|^{m+\frac{r^{\prime}-q^{\prime}}{r^{\prime} q^{\prime}}(3+\varepsilon)}\left[\hat{\rho}_{ \pm 0}\right]\right\|_{L^{p^{\prime}}(|k| \leq 1)} \\
\leq e^{-t / 2}\left\||k|^{m+\left(\frac{1}{q^{\prime}}-\frac{1}{r^{\prime}}\right)(3+\varepsilon)}\left[\hat{\rho}_{ \pm 0}\right]\right\|_{L^{p^{\prime}}(|k| \leq 1)} \\
\leq C e^{-t / 2}\left\|\nabla^{m+\left[3\left(\frac{1}{r}-\frac{1}{q}\right)\right]_{+}}\left[\hat{\rho}_{ \pm 0}\right]\right\|_{L^{r}} .
\end{aligned}
$$

To estimate the second part of the third term, we get

$$
\begin{aligned}
\||k|^{m} & \frac{1}{|k|} e^{\frac{-\theta t}{|k|^{2}}}\left[\hat{\rho}_{ \pm 0}, \hat{u}_{ \pm 0}, \hat{E}_{0}, \hat{B}_{0}\right] \|_{L^{q^{\prime}}}=\left(\left.\left.\int_{\mathbb{R}^{3}}|| k\right|^{m-1} e^{\frac{-\theta t}{|k|^{2}}}\left[\hat{u}_{ \pm 0}, \hat{E}_{0}, \hat{B}_{0}\right]\right|^{q^{\prime}}\right)^{1 / q^{\prime}} \\
& =\left(\left.\int_{\mathbb{R}^{3}}|| k\right|^{-\frac{r^{\prime}-q^{\prime}}{r^{\prime} q^{\prime}}(3+\varepsilon)}|k|^{m+\frac{r^{\prime}-q^{\prime}}{r^{\prime} q^{\prime}}(3+\varepsilon)} e^{\left.\left.\frac{-\theta t}{|k|^{2}}\left[\hat{u}_{ \pm 0}, \hat{E}_{0}, \hat{B}_{0}\right]\right|^{q^{\prime}}\right)^{1 / q^{\prime}}}\right. \\
& \leq\left\||k|^{-(3+\varepsilon)}\right\|^{-\frac{r^{\prime}-q^{\prime}}{r^{\prime} q^{\prime}}}\left\||k|^{m+\frac{r^{\prime}-q^{\prime}}{r^{\prime} q^{\prime}}(3+\varepsilon)} e^{\frac{-\theta t}{|k|^{2}}}\left[\hat{u}_{ \pm 0}, \hat{E}_{0}, \hat{B}_{0}\right]\right\|_{L^{r^{\prime}}(|k| \leq 1)} \\
& \leq e^{\frac{-\theta t}{|k|^{2}}}\left\||k|^{m+l+3\left(\frac{1}{q^{\prime}}-\frac{1}{r^{\prime}}\right)}|k|^{-(l+1)}\left[u_{ \pm 0}, E_{0}, B_{0}\right]\right\|_{L^{r^{\prime}}(|k| \leq 1)} \\
& \leq C(1+t)^{-\frac{l+1}{2}}\left\|\nabla^{m+\left[l+3\left(\frac{1}{r}-\frac{1}{q}\right)\right]_{+}}\left[u_{ \pm 0}, E_{0}, B_{0}\right]\right\|_{L^{r}}
\end{aligned}
$$

where we used

$$
\sup _{|k| \leq 1} \frac{1}{|k|^{l+1}} e^{\frac{-\theta(t+1)}{|k|^{2}}} \leq C(1+t)^{-\frac{l+1}{2}} .
$$

The proofs of (3.79) and (3.80) are similar.

Note that we transformed to the Fourier space to find the solution representation. Now we go back to the physical space $x(t)$ and obtain the decay rates for the solutions to the linearized equations. Fundamental properties of the system are given by the following corollary based on Theorem 3.6.

Corollary 3.1. Assume $U(t)=e^{t L} U_{0}$ is the solution of the initial value problem (3.1),(3.2) with initial data $\left[\rho_{ \pm 0}, u_{ \pm 0}, E_{0}, B_{0}\right]$ which satisfies (3.3). Then $U=\left[\rho_{ \pm}, u_{ \pm}, E, B\right]$ satisfies

$$
\left\{\begin{array}{l}
\left\|\rho_{ \pm}(t)\right\| \leq C e^{-t / 2}\left\|\left[\rho_{ \pm 0}, u_{ \pm 0}\right]\right\| \\
\left\|u_{ \pm}(t)\right\| \leq C e^{-t / 2}\left\|\left[\rho_{ \pm 0}\right]\right\|+C(1+t)^{-\frac{5}{4}}\left\|\left[u_{ \pm 0}, E_{0}, B_{0}\right]\right\|_{L^{1} \cap \dot{H}^{2}} \\
\|E(t)\| \leq C(1+t)^{-\frac{5}{4}}\left\|\left[u_{ \pm 0}, E_{0}, B_{0}\right]\right\|_{L^{1} \cap \dot{H}^{3}} \\
\|B(t)\| \leq C(1+t)^{-\frac{3}{4}}\left\|\left[u_{ \pm 0}, E_{0}, B_{0}\right]\right\|_{L^{1} \cap \dot{H}^{2}}
\end{array}\right.
$$




$$
\left\{\begin{array}{l}
\left\|\rho_{ \pm}(t)\right\|_{L^{\infty}} \leq C e^{-t / 2}\left\|\left[\rho_{ \pm 0}, u_{ \pm 0}\right]\right\|_{L^{2} \cap \dot{H}^{2}} \\
\left\|u_{ \pm}(t)\right\|_{L^{\infty}} \leq C e^{-t / 2}\left\|\left[\rho_{ \pm 0}\right]\right\|\left\|_{L^{1} \cap \dot{H}^{2}}+C(1+t)^{-2}\right\|\left[u_{ \pm 0}, E_{0}, B_{0}\right] \|_{L^{1} \cap \dot{H}^{5}} \\
\|E(t)\|_{L^{\infty}} \leq C(1+t)^{-2}\left\|\left[u_{ \pm 0}, E_{0}, B_{0}\right]\right\|_{L^{1} \cap \dot{H}^{6}} \\
\|B(t)\|_{L^{\infty}} \leq C(1+t)^{-\frac{3}{2}}\left\|\left[u_{ \pm 0}, E_{0}, B_{0}\right]\right\|_{L^{1} \cap \dot{H}^{5}}
\end{array}\right.
$$

and

$$
\left\{\begin{array}{l}
\|\nabla B(t)\| \leq C(1+t)^{-\frac{5}{4}}\left\|\left[u_{ \pm 0}, E_{0}, B_{0}\right]\right\|_{L^{1} \cap \dot{H}^{4}} \\
\left\|\nabla^{N}[E(t), B(t)]\right\| \leq C(1+t)^{-\frac{5}{4}}\left\|\left[u_{ \pm 0}, E_{0}, B_{0}\right]\right\|_{L^{1} \cap \dot{H}^{N+3}}
\end{array}\right.
$$

Proof: The results of Corollary 3.1 are particular cases of Theorem 3.6.

For example we explain how we get the decay rates for $u_{ \pm}$. From equation (3.78) for $L^{2}$ take $q=2, p=1, m=0, l=3 / 2$ and $r=2$

$$
\begin{aligned}
\left\|\nabla^{m} u_{ \pm}(t)\right\|_{L^{2}} \leq C e^{-\frac{t}{2}}\left(\left\|\rho_{ \pm 0}\right\|_{L^{1}}+\left\|\nabla^{\left[\frac{3}{2}\right]+1}\left[\rho_{ \pm 0}, u_{ \pm 0}\right]\right\|_{L^{1}}\right) \\
+C(1+t)^{-\frac{5}{4}}\left\|\left[u_{ \pm 0}, E_{0}, B_{0}\right]\right\|_{L^{1}} \\
+C(1+t)^{-\frac{5}{4}}\left\|\nabla^{\left[1+\frac{1}{2}\right]_{+1}}\left[u_{ \pm 0}, E_{0}, B_{0}\right]\right\|_{L^{1}}
\end{aligned}
$$

Thus

$$
\left\|u_{ \pm}(t)\right\|_{L^{2}} \leq C e^{-t / 2}\left\|\left[\rho_{ \pm 0}\right]\right\|+C(1+t)^{-\frac{5}{4}}\left\|\left[u_{ \pm 0}, E_{0}, B_{0}\right]\right\|_{L^{1} \cap \dot{H}^{2}}
$$

Now for $L^{\infty}$ take $q=\infty, p=1, m=0, l=3$ and $r=2$

$$
\begin{aligned}
\left\|\nabla^{m} u_{ \pm}(t)\right\|_{L^{\infty}} & \left.\leq C e^{-\frac{t}{2}}\left(\left\|\rho_{ \pm 0}\right\|_{L^{1}}+\| \nabla^{\left[\frac{3}{2}\right]}\right]_{+1}\left[\rho_{ \pm 0}, u_{ \pm 0}\right] \|_{L^{2}}\right) \\
& +C(1+t)^{-2}\left\|\left[u_{ \pm 0}, E_{0}, B_{0}\right]\right\|_{L^{1}} \\
& +C(1+t)^{-2}\left\|\nabla^{\left[4+\frac{1}{2}\right]_{+1}}\left[u_{ \pm 0}, E_{0}, B_{0}\right]\right\|_{L^{2}}
\end{aligned}
$$

Thus

$$
\left\|u_{ \pm}(t)\right\|_{L^{\infty}} \leq C e^{-t / 2}\left\|\left[\rho_{ \pm 0}\right]\right\|_{L^{1} \cap \dot{H}^{2}}+C(1+t)^{-2}\left\|\left[u_{ \pm 0}, E_{0}, B_{0}\right]\right\|_{L^{1} \cap \dot{H}^{5}}
$$




\section{Chapter 4}

\section{The decay rates for nonlinear systems.}

\subsection{Decay rates for system 2.2.}

In this section, we apply the linear $L^{p}-L^{q}$ time-decay property of the homogeneous system (3.1) to the nonlinear case. Throughout this section, we suppose that $U=$ $\left[\rho_{ \pm}, u_{ \pm}, E, B\right]$ is the solution to the Cauchy problem (2.2) satisfying (2.3).

By Duhamel's Principle, the solution $U$ can be formally written as

$$
U=e^{t L} U_{0}+\int_{0}^{t} e^{(t-s) L}\left[g_{1}(s), g_{2}(s), g_{3}(s), 0\right] d s,
$$

where $e^{t L} U_{0}$ is as defined in section 3.2 and

$$
\left\{\begin{array}{l}
g_{1}=-\nabla \cdot\left(\rho_{ \pm} u_{ \pm}\right) \\
g_{2}=-u_{ \pm} \cdot \nabla u_{ \pm}-A_{ \pm} \gamma\left[\left(\rho_{ \pm}+1\right)^{\gamma-2}-1\right] \nabla \rho_{ \pm} \pm\left(u_{ \pm} \times B\right)+\alpha_{ \pm}\left(u_{ \pm}-u_{\mp}\right), \\
g_{3}=\rho_{-} u_{-}-\rho_{+} u_{+} .
\end{array}\right.
$$

Remark 4.1. Note that in (4.1), $\left[g_{1}(s), g_{2}(s), g_{3}(s), 0\right]$ satisfies the compatibility condition (3.3). Thus $e^{(t-s) L}$ acts on $\left[g_{1}(s), g_{2}(s), g_{3}(s), 0\right]$ for all $0 \leq s \leq t$.

Proposition 4.1. Suppose initial data $V_{0}=\left[\sigma_{ \pm 0}, v_{ \pm 0}, \tilde{E}_{0}, \tilde{B_{0}}\right]$ satisfies (2.4). If $\varepsilon_{N+2}\left(V_{0}\right)>0$ is small enough, then the solution $V=\left[\sigma_{ \pm}, v_{ \pm}, \tilde{E}, \tilde{B}\right]$ satisfies

$$
\|V(t)\|_{N} \leq C_{\varepsilon_{N+2}}\left(V_{0}\right)(1+t)^{-3 / 4}
$$

for any $t>0$. Moreover, $\varepsilon_{N+6}\left(V_{0}\right)>0$ begin sufficiently small implies the solution $V=\left[\sigma_{ \pm}, v_{ \pm}, \tilde{E}, \tilde{B}\right]$ satisfies

$$
\|\nabla V(t)\|_{N-1} \leq C_{\varepsilon_{N+6}}\left(V_{0}\right)(1+t)^{-5 / 4}
$$

for any $t \geq 0$ 
Lemma 4.1. Suppose $V=\left[\sigma_{ \pm}, v_{ \pm}, E, B\right]$ is the solution of the initial value problem (2.2), (2.3) with initial data $V_{0}=\left[\sigma_{ \pm}, v_{ \pm}, \tilde{E}_{0}, \tilde{B_{0}}\right]$ satisfying (2.4) as described in Proposition 2.1. If $\mathcal{E}_{N}\left(V_{0}\right)$ is sufficiently small, then

$$
\frac{d}{d t} \varepsilon_{N}(V(t))+\lambda \mathcal{D}_{N}(V(t)) \leq 0 .
$$

Proof: Let $l \geqslant 0$. Multiplying equation (4.5) by $(1+t)^{l}$ we get

$$
\frac{d}{d t} \varepsilon_{N}(V(t))(1+t)^{l}+\lambda \mathcal{D}_{N}(V(t))(1+t)^{l} \leq 0
$$

Integrating over $[0,1]$, we find

$$
\int_{0}^{t} \frac{d}{d t} \mathcal{E}_{N}(V(s))(1+s)^{l} d s+\lambda \int_{0}^{t}(1+s)^{l} \mathcal{D}_{N}(V(s)) d s \leq 0
$$

which implies

$$
(1+t)^{l} \mathcal{E}_{N}(V(t))+\lambda \int_{0}^{t}(1+s)^{l} \mathcal{D}_{N}(V(s)) d s \leq \mathcal{E}_{N}\left(V_{0}\right)+l \int_{0}^{t}(1+s)^{l-1} \mathcal{E}_{N}(V(s)) d s .
$$

Recalling

$$
\mathcal{E}_{N}\left(V(t) \leq C\left(\mathcal{D}_{N}(V(t))+\|\tilde{B}\|^{2}+\left\|\sigma_{+}+\sigma_{-}\right\|^{2}\right)\right.
$$

we obtain

$$
\begin{aligned}
(1+t)^{l} \mathcal{E}_{N}(V(t)) & +\lambda \int_{0}^{t}(1+s)^{l} \mathcal{D}_{N}(V(s)) d s \\
& \leq \mathcal{E}_{N}\left(V_{0}\right)+C l \int_{0}^{t}(1+s)^{l-1}\left(\mathcal{D}_{N}(V(t))+\|\tilde{B}\|^{2}+\left\|\sigma_{+}+\sigma_{-}\right\|^{2}\right) d s \\
& +C l \int_{0}^{t}(1+s)^{l-1} \mathcal{D}_{N+1}(V(s)) d s .
\end{aligned}
$$

Similarly,

$$
\begin{aligned}
(1+t)^{l-1} & \mathcal{E}_{N+1}(V(t))+\lambda \int_{0}^{t}(1+s)^{l-1} \mathcal{D}_{N+1}(V(s)) d s \\
& \leq \mathcal{E}_{N+1}\left(V_{0}\right)+C l-1 \int_{0}^{t}(1+s)^{l-2}\left(\mathcal{D}_{N}(V(t))+\|\tilde{B}\|^{2}+\left\|\sigma_{+}+\sigma_{-}\right\|^{2}\right) d s \\
& +C l+1 \int_{0}^{t}(1+s)^{l-2} \mathcal{D}_{N+2}(V(s)) d s .
\end{aligned}
$$


and

$$
\lambda \int_{0}^{t} \mathcal{D}_{N+2}(V(s)) d s \leq \mathcal{E}_{N+2}\left(V_{t}\right)+\lambda \int_{0}^{t} \mathcal{D}_{N+2}(V(s)) d s \leq \mathcal{E}_{N+2}\left(V_{0}\right)
$$

for $1 \leq l \leq 2$. Thus

$$
\begin{aligned}
(1+t)^{l} \mathcal{E}_{N}(V(t)) & +\lambda \int_{0}^{t}(1+s)^{l} \mathcal{D}_{N}(V(s)) d s \\
& \leq \mathcal{E}_{N}\left(V_{0}\right)+C \int_{0}^{t}(1+s)^{l-1}\left(\|\tilde{B}\|^{2}+\left\|\sigma_{+}+\sigma_{-}\right\|^{2}\right)
\end{aligned}
$$

To estimate the term on the right hand side of (4.13), define

$$
\mathcal{E}_{N, \infty} \leq(1+s)^{3 / 2} \mathcal{E}_{N}(V(s)) .
$$

Applying Duhamel's principle for $t \geq 0$ along with the linear estimate on B from (3.83) to (4.1), we get

$$
\|B(t)\| \leq(1+t)^{-3 / 4}\left\|\left[u_{ \pm 0}, E_{0}, B_{0}\right]\right\|_{L^{1} \cap \dot{H}^{2}}+C \int_{0}^{t}(1+s)^{-3 / 4}\left\|\left[g_{2}(s), g_{3}(s)\right]\right\|_{L^{1} \cap \dot{H}^{2}}
$$

for all $0 \leq s \leq t$.

Since

$$
\begin{gathered}
\left\|g_{2}(s)\right\|_{L^{1}}=\int\left|--u_{ \pm} \cdot \nabla u_{ \pm}-A_{ \pm} \gamma\left[\left(\rho_{ \pm}+1\right)^{\gamma-2}-1\right] \nabla \rho_{ \pm} \pm\left(u_{ \pm} \times B\right)+\alpha_{ \pm}\left(u_{ \pm}-u_{\mp}\right)\right| d s \\
\leq \int\left|-u_{ \pm} \cdot \nabla u_{ \pm}\right| d s+\int\left|-u_{ \pm} \times B\right| d s+\int\left|\gamma\left[\left(1+\rho_{ \pm}\right)^{\gamma-2}-1\right] \nabla \rho_{ \pm}\right| d s
\end{gathered}
$$

we can apply the Sobolev imbedding theorem along with the Hölder and Cauchy inequalities to obtain

$$
\left\|g_{2}(s)\right\|_{L^{1}} \leq C\left(\left\|\nabla u_{ \pm}\right\|+\left\|u_{ \pm}\right\|+\|B\|+\left\|\nabla \rho_{ \pm}\right\|\right)
$$

and

$$
\left\|g_{3}(s)\right\|_{L^{1}}=\int\left|\rho_{ \pm} u_{ \pm}\right| d s
$$

Thus,

$$
\left\|g_{3}(s)\right\|_{L^{1}} \leq C\left(\left\|\rho_{ \pm}\right\|+\left\|u_{ \pm}\right\|\right)
$$

Therefore, we get

$$
\left\|g_{2}(t)\right\|_{\dot{H}^{2}}=\left(\sum_{\alpha=1}^{2} \mid D^{\alpha}\left(-u_{ \pm} . \nabla u_{ \pm}-A_{ \pm} \gamma\left[\left(\rho_{ \pm}+1\right)^{\gamma-2}-1\right] \nabla \rho_{ \pm} \pm\left(u_{ \pm} \times B\right)+\alpha_{ \pm}\left(u_{ \pm}-u_{\mp}\right) \mid\right)\right.
$$


Thus,

$$
\left\|g_{2}(t)\right\|_{\dot{H}^{2}} \leq\|\nabla U(t)\|_{2}\left\|u_{ \pm}, B(t)\right\|_{L^{\infty}}
$$

and

$$
\left\|g_{3}(s)\right\|_{\dot{H}^{2}}=\left(\sum_{\alpha=1}^{2} \int\left|D^{\alpha}\left(\rho_{ \pm} u_{ \pm}\right)\right|^{2} d s\right)^{1 / 2}
$$

Thus,

$$
\left\|g_{3}(t)\right\|_{\dot{H}^{2}} \leq\|\nabla U(t)\|_{2}\left\|\left[\rho_{ \pm}, u_{ \pm}\right]\right\|_{L^{\infty}} .
$$

Therefore,

$$
\left\|\left[g_{2}(s), g_{3}(s)\right]\right\|_{L^{1} \cap \dot{H}^{2}} \leq C \mathcal{E}_{N}(U(s)) .
$$

Notice that

$$
\mathcal{E}_{N}(U(s)) \leq C \mathcal{E}_{N}(V(\sqrt{\gamma} s))
$$

From (4.14)

$$
\varepsilon_{N}(V(\sqrt{\gamma} S)) \leq(1+\sqrt{\gamma} S)^{-3 / 2} \mathcal{E}_{N, \infty}(V(\sqrt{\gamma} t)),
$$

for any $0 \leq s \leq t$.

Then

$$
\left\|\left[g_{2}(s), g_{3}(s)\right]\right\|_{L^{1} \cap \dot{H}^{2}} \leq C(1+\sqrt{\gamma} s)^{-3 / 2} \mathcal{E}_{N, \infty}(V(\sqrt{\gamma} t))
$$

Substituting equation (4.24) in equation (4.15) we get

$$
\begin{aligned}
\|B(t)\| & \leq(1+t)^{-3 / 4}\left(\left\|\left[u_{ \pm 0}, E_{0}, B_{0}\right]\right\|_{L^{1} \cap \dot{H}^{2}}+C(1+s)^{-3 / 2} \mathcal{E}_{N, \infty}(V(\sqrt{\gamma} t))\right) \\
& \leq(1+t)^{-3 / 4}\left(\left\|\left[u_{ \pm 0}, E_{0}, B_{0}\right]\right\|_{L^{1} \cap \dot{H}^{2}}+\varepsilon_{N, \infty}(V(\sqrt{\gamma} t))\right),
\end{aligned}
$$

which implies

$$
\|\tilde{B}(t)\|^{2} \leq(1+t)^{-3 / 2}\left(\left\|\left[v_{ \pm 0}, \tilde{E}_{0}, \tilde{B}_{0}\right]\right\|_{L^{1} \cap \dot{H}^{2}}^{2}+\left(\mathcal{E}_{N, \infty}(V(\sqrt{\gamma} t))\right)^{2}\right) .
$$

Finally, we need to show the uniform-in-time bound of $\mathcal{E}_{N, \infty}(V(t))$ which implies the decay rates of the energy functional $\mathcal{E}_{N}(V(t))$. Using $l=\frac{3}{2}+\varepsilon$ in (4.13) with 
$\varepsilon>0$ sufficiently small and using (4.25) we get

$$
\begin{aligned}
(1+t)^{\frac{3}{2}+\varepsilon} \mathcal{E}_{N}(V(t))+ & \lambda \int_{0}^{t}(1+s)^{\frac{3}{2}+\varepsilon} \mathcal{D}_{N}(V(s)) d s \\
\leq & C \mathcal{E}_{N+2}\left(V_{0}\right)+C \int_{0}^{t}(1+s)^{\frac{1}{2}+\varepsilon}\left(\|\tilde{B}\|^{2}+\left\|\sigma_{+}+\sigma_{-}\right\|^{2}\right) \\
\leq & C \mathcal{E}_{N+1}\left(V_{0}\right)+(1+t)^{\frac{3}{2}+\varepsilon}\left(C(1+t)^{-3 / 2}\left\|\left[v_{ \pm 0}, \tilde{E}_{0}, \tilde{B}_{0}\right]\right\|_{L^{1} \cap \dot{H}^{2}}^{2}\right. \\
& \left.\quad+C(1+s)^{-3 / 2}\left(\mathcal{E}_{N, \infty}(V(t))\right)^{2}\right) \\
& \leq C \mathcal{E}_{N+2}\left(V_{0}\right)+C(1+t)^{\varepsilon}\left(\left\|\left[v_{ \pm 0}, \tilde{E}_{0}, \tilde{B}_{0}\right]\right\|_{L^{1} \cap \dot{H}^{2}}^{2}+\left(\mathcal{E}_{N, \infty}(V(t))\right)^{2}\right),
\end{aligned}
$$

which implies

$$
(1+t)^{\frac{3}{2}} \mathcal{E}_{N}(V(t)) \leq C\left(\mathcal{E}_{N+2}\left(V_{0}\right)+\left\|\left[v_{ \pm 0}, \tilde{E}_{0}, \tilde{B}_{0}\right]\right\|_{L^{1}}^{2}+\left(\mathcal{E}_{N, \infty}(V(t))\right)^{2}\right)
$$

and

$$
(1+t)^{\frac{3}{2}}\left(\varepsilon_{N, \infty}(V(t))\right) \leq C\left(\varepsilon_{N+2}\left(V_{0}\right)^{2}+\left(\varepsilon_{N, \infty}(V(t))\right)^{2}\right) .
$$

Now from the definition (2.16), and since $\varepsilon_{N+2}\left(V_{0}\right)>0$ is sufficiently small, $\mathcal{E}_{N, \infty}(V(t)) \leq$ $\varepsilon_{N+2}\left(V_{0}\right)^{2}$ holds for any $t \geq 0$ we get

$$
\|V(t)\|_{N} \leq C\left(\mathcal{E}_{N}(V(t))^{1 / 2} \leq C \varepsilon_{N+2}\left(V_{0}\right)(1+t)^{-3 / 4}\right.
$$

that is the proof of (4.3) in Proposition 4.1.

\subsection{Time rate for the high-order energy functional}

In this section, we determine the time-decay estimates of the high-order energy functional $\|\nabla V\|_{N}^{2}$, that is the proof (4.4) of Proposition 4.1. To do so, we investigate the time-decay estimates on $\|\nabla \tilde{B}\|$ and $\left\|\nabla^{N}[\tilde{E}, \tilde{B}]\right\|$ using the following lemma.

Lemma 4.2. Suppose $V=\left[\sigma_{ \pm}, v_{ \pm}, \tilde{E}, \tilde{B}\right]$ is the solution to the Cauchy proplem (2.2) and (2.3) with initial condition $V_{0}=\left[\sigma_{0}, v_{0}, \tilde{E}_{0}, \tilde{B_{0}}\right]$ satisfying (2.4) as obtained in Proposition 2.1. If $\mathcal{E}_{N}\left(V_{0}\right)$ is sufficiently small, then there exists the high-order energy functional $\mathcal{E}_{N}^{h}(\cdot)$ and the high-order dissipation rate $\mathcal{D}_{N}^{h}(\cdot)$ such that for all $\geq 0$

$$
\frac{d}{d t} \varepsilon_{N}^{h}(V(t))+\lambda \mathcal{D}_{N}^{h}(V(t)) \leq C\|\nabla \tilde{B}\|^{2}
$$


By comparing the definitions of $\mathcal{E}_{N}^{h}(V(t))$ (2.9) and $\mathcal{D}_{N}^{h}(V(t))$ (2.13) and based on lemma 4.2 the equation (4.31) we have

$$
\frac{d}{d t} \varepsilon_{N}^{h}(V(t))+\lambda \varepsilon_{N}^{h}(V(t)) \leq C\left(\|\nabla \tilde{B}\|^{2}+\left\|\nabla^{N}[\tilde{E}, \tilde{B}]\right\|+\left\|\nabla\left(\sigma_{+}+\sigma_{-}\right)\right\|^{2}\right)
$$

which implies

$\mathcal{E}_{N}^{h}(V(t)) \leq e^{-\lambda t} \mathcal{E}_{N}^{h}\left(V_{0}\right)+C \int_{0}^{t} e^{-\lambda(t-s)}\left(\|\nabla \tilde{B}(s)\|^{2}+\| \nabla^{N}[\tilde{E}, \tilde{B}]\left(s\|+\| \nabla\left(\sigma_{+}+\sigma_{-}\right)(s) \|^{2}\right)\right.$.

Now, we estimate the time integral term om the right-hand side of the inequality (4.32), we have the next lemma.

Lemma 4.3. Suppose $V=\left[\sigma_{ \pm}, v_{ \pm}, \tilde{E}, \tilde{B}\right]$ is the solution of the initial value proplem (2.2) and (2.3) with initial condition $V_{0}=\left[\sigma_{0}, v_{0}, \tilde{E}_{0}, \tilde{B}_{0}\right]$ satisfying (2.4) as obtained in Proposition 2.1. If $\varepsilon_{N+6}\left(V_{0}\right)$ is sufficiently small, where defined in (2.16), then for all $t \geq 0$

$$
\|\nabla \tilde{B}(t)\|^{2}+\left\|\nabla^{N}[\tilde{E}(t), \tilde{B}(t)]\right\|^{2}+\left\|\nabla^{N}\left(\sigma_{+}(t)+\sigma_{-}(t)\right)\right\|^{2} \leq \varepsilon_{N+6}\left(V_{0}\right)^{2}(1+t)^{\frac{5}{2}} .
$$

The proofs of Lemma 4.2 and Lemma 4.3 are analogous to those of Lemmas 5.1 and 5.2 in [2].

Now we suppose that the above lemma is true. Then by putting (4.32) into (4.31) we get

$$
\varepsilon_{N}^{h}(V(t)) \leq e^{-\lambda t} \varepsilon_{N}^{h}\left(V_{0}\right)+C \varepsilon_{N+6}\left(V_{0}\right)^{2}(1+t)^{\frac{5}{2}},
$$

which (4.4) in Proposition 4.1 holds.

\subsection{Decay rate in $L^{q}$}

In this section we determine the decay rates in $L^{q}, 2 \leq q \leq+\infty$, of (1.4), (1.5), and (1.6) for solutions $U=\left[\rho_{ \pm}, u_{ \pm}, E, B\right]$ to the Cauchy problem (3.1) and (3.2). Suppose that $\varepsilon_{13}\left(V_{0}\right)$ is sufficiently small. In addition, for $N \geq 4$, Proposition 4.1 shows that if $\varepsilon_{N+2}\left(V_{0}\right)$ is sufficiently small then

$$
\|U(t)\|_{N} \leq C \varepsilon_{N+2}\left(V_{0}\right)(1+t)^{-\frac{3}{4}}
$$

and if $\varepsilon_{N+6}\left(V_{0}\right)$ is sufficiently small then

$$
\|\nabla U(t)\|_{N-1} \leq C \varepsilon_{N+6}\left(V_{0}\right)(1+t)^{-\frac{5}{4}} .
$$


We establish the estimates on $B,\left[u_{ \pm}, E\right]$ and $\rho_{ \pm}$as follows.

To estimate the $L^{2}$ decay rate on $\|B\|_{L^{q}}$, note that from (4.34) we obtain

$$
\|B(t)\| \leq C \varepsilon_{6}\left(V_{0}\right)(1+t)^{-\frac{3}{4}} .
$$

To estimate the $L^{\infty}$ decay rate, note that the $L^{\infty}$ estimates on $B$ in (3.84) to (4.1) give $\|B(t)\|_{L^{\infty}} \leq C(1+t)^{-\frac{3}{2}}\left\|\left[u_{ \pm 0}, E_{0}, B_{0}\right]\right\|_{L^{1} \cap \dot{H}^{5}}+C \int_{0}^{t}(1+t-s)^{-\frac{3}{2}}\left\|\left[g_{2}(s), g_{3}(s)\right]\right\|_{L^{1} \cap \dot{H}^{5}} d s$.

From (4.34) we obtain

$$
\left\|\left[g_{2}(s), g_{3}(s)\right]\right\|_{L^{1} \cap \dot{H}^{5}} \leq C\|U(t)\|_{6}^{2} \leq C \varepsilon_{8}^{2}\left(V_{0}\right)(1+t)^{-\frac{3}{2}},
$$

and thus

$$
\|B(t)\|_{L^{\infty}} \leq C \varepsilon_{8}\left(V_{0}\right)(1+t)^{-\frac{3}{2}} .
$$

Moreover, by $L^{2}-L^{\infty}$ interpolation,

$$
\|B(t)\|_{L^{q}} \leq C \varepsilon_{8}\left(V_{0}\right)(1+t)^{-\frac{3}{2}+\frac{3}{2 q}}
$$

for $2 \leq q \leq \infty$.

To estimate $\left\|u_{ \pm}, E\right\|_{L^{q}}$, note that for the $L^{2}$ decay rate we can utilize the $L^{2}$ estimate on $u_{ \pm}$and $E$ in (3.83) to (4.1). That is,

$$
\begin{aligned}
\left\|u_{ \pm}(t)\right\| \leq & C(1+t)^{-\frac{5}{4}}\left(\left\|\rho_{ \pm 0}\right\|+\left\|\left[u_{ \pm 0}, E_{0}, B_{0}\right]\right\|_{L^{1} \cap \dot{H}^{2}}\right) \\
& +C \int_{0}^{t}(1+t-s)^{-\frac{5}{4}}\left(\left\|g_{1}(s)\right\|+\left\|\left[g_{2}(s), g_{3}(s)\right]\right\|_{L^{1} \cap \dot{H}^{2}}\right) d s
\end{aligned}
$$

and

$$
\begin{aligned}
\|E(t)\| \leq C & (1+t)^{-\frac{5}{4}}\left\|\left[u_{ \pm 0}, E_{0}, B_{0}\right]\right\|_{L^{1} \cap \dot{H}^{3}} \\
& +C \int_{0}^{t}(1+t-s)^{-\frac{5}{4}}\left\|\left[g_{2}(s), g_{3}(s)\right]\right\|_{L^{1} \cap \dot{H}^{3}} d s .
\end{aligned}
$$

From (4.34),

$$
\left\|g_{1}(t)\right\|+\left\|\left[g_{2}(t), g_{3}(t)\right]\right\|_{L^{1} \cap \dot{H}^{3}} \leq\|U(t)\|_{4}^{2} \leq C \varepsilon_{6}^{2}\left(V_{0}\right)(1+t)^{-\frac{3}{2}},
$$

and it therefore holds that

$$
\left\|u_{ \pm}(t), E(t)\right\| \leq C \varepsilon_{6}\left(V_{0}\right)(1+t)^{-\frac{5}{4}} .
$$


For the $L^{\infty}$ decay rate, we can utilize the $L^{\infty}$ estimates on $u_{ \pm}$and $E$ in (3.84) to (4.1). That is,

$$
\begin{aligned}
\left\|u_{ \pm}(t)\right\|_{L^{\infty}} \leq C(1+t)^{-2}\left(\left\|\rho_{ \pm 0}\right\|_{L^{2} \cap \dot{H}^{2}}+\left\|\left[u_{ \pm 0}, E_{0}, B_{0}\right]\right\|_{L^{1} \cap \dot{H}^{5}}\right) \\
+C \int_{0}^{t}(1+t-s)^{-2}\left(\left\|g_{1}(s)\right\|_{L^{2} \cap \dot{H}^{2}}+\left\|\left[g_{2}(s), g_{3}(s)\right]\right\|_{L^{1} \cap \dot{H}^{5}}\right) d s
\end{aligned}
$$

and

$$
\begin{aligned}
\|E(t)\|_{L^{\infty}} \leq C(1+t)^{-2}\left\|\left[u_{ \pm 0}, E_{0}, B_{0}\right]\right\|_{L^{1} \cap \dot{H}^{6}} & \\
& +C \int_{0}^{t}(1+t-s)^{-2}\left\|\left[g_{2}(s), g_{3}(s)\right]\right\|_{L^{1} \cap \dot{H}^{6}} d s .
\end{aligned}
$$

Since

$$
\left\|g_{1}(t)\right\|_{L^{2} \cap \dot{H}^{2}}+\left\|\left[g_{2}(t), g_{3}(t)\right]\right\|_{\dot{H}^{5} \cap \dot{H}^{6}} \leq\|\nabla U(t)\|_{6}^{2} \leq C \varepsilon_{13}^{2}\left(V_{0}\right)(1+t)^{-\frac{5}{2}}
$$

and

$$
\begin{aligned}
\left\|\left[g_{2}(t), g_{3}(t)\right]\right\|_{L^{1}} & \leq C\|U(t)\|\left(\left\|u_{ \pm}(t)\right\|+\|\nabla U(t)\|\right) \\
& \leq C\left[\varepsilon_{6}\left(V_{0}\right)(1+t)^{-\frac{3}{4}}\right] \cdot\left[\varepsilon_{10}\left(V_{0}\right)(1+t)^{-\frac{5}{4}}\right] \leq C \varepsilon_{10}^{2}\left(V_{0}\right)(1+t)^{-2}
\end{aligned}
$$

it holds that

$$
\left\|u_{ \pm}(t), E(t)\right\|_{L^{\infty}} \leq C \varepsilon_{13}\left(V_{0}\right)(1+t)^{-2} .
$$

Moreover, by $L^{2}-L^{\infty}$ interpolation,

$$
\left\|u_{ \pm}(t), E(t)\right\|_{L^{q}} \leq C \varepsilon_{13}\left(V_{0}\right)(1+t)^{-2+\frac{3}{2 q}}
$$

for $2 \leq q \leq \infty$.

To estimate the $L^{2}$ decay rate on $\left\|\rho_{ \pm}\right\|_{L^{q}}$, we utilize the $L^{2}$ estimate on $\rho_{ \pm}$in (3.83) to (4.1). We get

$$
\left\|\rho_{ \pm}(t)\right\| \leq C e^{-\frac{t}{2}}\left\|\left[\rho_{ \pm 0}, u_{ \pm 0}\right]\right\|+C \int_{0}^{t} e^{-\frac{(t-s)}{2}}\left\|\left[g_{1}(s), g_{2}(s)\right]\right\| d s .
$$

Since

$$
\begin{aligned}
\left\|\left[g_{1}(t), g_{2}(t)\right]\right\| & \leq C\left(\|\nabla U(t)\|_{1}^{2}+\left\|u_{ \pm}(t)\right\| \cdot\|B(t)\|_{L^{\infty}}\right) \\
& \leq C \varepsilon_{10}^{2}\left(V_{0}\right)(1+t)^{-\frac{5}{2}}
\end{aligned}
$$

(4.38) implies the slower decay estimate

$$
\left\|\rho_{ \pm}(t)\right\| \leq C \varepsilon_{10}\left(V_{0}\right)(1+t)^{-\frac{5}{2}}
$$


Moreover, after estimating $\left\|\left[g_{1}(t), g_{2}(t)\right]\right\|$ and utilizing the previous slower decay estimate to obtain

$$
\begin{aligned}
\left\|\left[g_{1}(t), g_{2}(t)\right]\right\| & \leq C\left\|u_{ \pm}(t)\right\|_{L^{\infty}}\left(\left\|\nabla \rho_{ \pm}(t)\right\|+\left\|\nabla u_{ \pm}(t)\right\|+\|B(t)\|\right) \\
& \leq C\left\|\rho_{ \pm}(t)\right\|\left(\left\|\nabla \rho_{ \pm}(t)\right\|_{2}+\left\|\nabla u_{ \pm}(t)\right\|_{2}\right) \leq C \varepsilon_{13}\left(V_{0}\right)^{2}(1+t)^{-\frac{11}{4}}
\end{aligned}
$$

it follows from (4.38) that

$$
\left\|\rho_{ \pm}(t)\right\| \leq C \varepsilon_{13}\left(V_{0}\right)(1+t)^{-\frac{11}{4}}
$$

For the $L^{\infty}$ decay rate, we utilize the $L^{\infty}$ estimates on $\rho_{ \pm}$in (3.84) to (4.1). We have

$$
\left\|\rho_{ \pm}(t)\right\|_{L^{\infty}} \leq C e^{\frac{t}{2}}\left\|\rho_{ \pm 0}, u_{ \pm}\right\|_{L^{2} \cap \dot{H}^{2}}+C \int_{0}^{t} e^{-\frac{(t-s)}{2}}\left\|\left[g_{1}(s), g_{2}(s)\right]\right\|_{L^{2} \cap \dot{H}^{2}} d s
$$

and it is simple to check that

$$
\begin{aligned}
\left\|\left[g_{1}(t), g_{2}(t)\right]\right\|_{L^{2} \cap \dot{H}^{2}} & \leq C\|\nabla U(t)\|_{4}\left(\left\|\rho_{ \pm}(t)\right\|+\left\|u_{ \pm}(t), B(t)\right\|_{L^{\infty}}+\left\|\nabla\left[\rho_{ \pm}(t), u_{ \pm}(t)\right]\right\|_{L^{\infty}}\right) \\
& \leq C \varepsilon_{13}\left(V_{0}\right)(1+t)^{-\frac{11}{4}}
\end{aligned}
$$

Applying (4.41), we obtain

$$
\left\|\rho_{ \pm}(t)\right\|_{L^{\infty}} \leq C \varepsilon_{13}\left(V_{0}\right)(1+t)^{-\frac{11}{4}}
$$

Thus, by $L^{2}-L^{\infty}$ interpolation,

$$
\left\|\rho_{ \pm}(t)\right\|_{L^{q}} \leq C \varepsilon_{13}\left(V_{0}\right)(1+t)^{-\frac{11}{4}}
$$

for $2 \leq q \leq \infty$. Note that (4.36), (4.37) and (4.43), correspond to (1.6), (1.5) and (1.4), respectively.

Now, from (4.1) let $G(t) * u_{ \pm 0}=e^{t L} U_{0}$. For the $L^{2}$ decay rate, by applying (4.1) and (7.4) we have

$$
\left\|U-e^{t L} U_{0}\right\|^{2}=\left\|\int_{0}^{t} e^{(t-s) L}\left[g_{1}(s), g_{2}(s), g_{3}(s), 0\right] d s\right\|^{2} \leq \int_{0}^{t} e^{(t-s) L}\left\|\left[g_{1}(s), g_{2}(s), g_{3}(s), 0\right]\right\|^{2} d s,
$$

since

$$
\begin{aligned}
\left\|g_{1}(t)\right\|_{L^{2}}^{2}= & \left\|\int_{0}^{t} e^{(t-s) L}\left(-\nabla \cdot\left(\rho_{ \pm} u_{ \pm}\right)\right) d s\right\|^{2} \\
& \leq \int_{0}^{t} e^{(t-s) L}\left\|\nabla \cdot\left(\rho_{ \pm} u_{ \pm}\right)\right\|^{2} d s \\
& \leq C \varepsilon_{10}^{2}\left(V_{0}\right)(1+t)^{-\frac{5}{2}}
\end{aligned}
$$




$$
\begin{gathered}
\left\|g_{2}(t)\right\|_{L^{2}}^{2}=\left\|\int_{0}^{t} e^{(t-s) L}\left(-u_{ \pm} \cdot \nabla u_{ \pm}-A_{ \pm} \gamma\left[\left(\rho_{ \pm}+1\right)^{\gamma-2}-1\right] \nabla \rho_{ \pm} \pm\left(u_{ \pm} \times B\right)+\alpha_{ \pm}\left(u_{ \pm}-u_{\mp}\right)\right) d s\right\|^{2} \\
\leq \int_{0}^{t} e^{(t-s) L}\left\|-u_{ \pm} \cdot \nabla u_{ \pm}-A_{ \pm} \gamma\left[\left(\rho_{ \pm}+1\right)^{\gamma-2}-1\right] \nabla \rho_{ \pm} \pm\left(u_{ \pm} \times B\right)+\alpha_{ \pm}\left(u_{ \pm}-u_{\mp}\right)\right\|^{2} d s \\
\leq C \varepsilon_{10}^{2}\left(V_{0}\right)(1+t)^{-\frac{5}{2}} \\
\left\|g_{3}(t)\right\|_{L^{2}}^{2}=\left\|\int_{0}^{t} e^{(t-s) L}\left(\rho_{-} u_{-}-\rho_{+} u_{+}\right) d s\right\|^{2} \\
\leq \int_{0}^{t} e^{(t-s) L}\left\|\rho_{-} u_{-}-\rho_{+} u_{+}\right\|^{2} d s \\
\leq C \varepsilon_{10}^{2}\left(V_{0}\right)(1+t)^{-\frac{5}{2}} .
\end{gathered}
$$

Therefore,

$$
\left\|U-e^{t L} U_{0}\right\|_{L^{2}} \leq C \varepsilon_{10}\left(V_{0}\right)(1+t)^{-\frac{5}{2}} .
$$

For the $L^{\infty}$ decay rate, by applying (4.1) and (7.4) we get

$$
\begin{gathered}
\left\|g_{1}(t)\right\|_{L^{\infty}}=\sup _{0 \leq s \leq t}\left|\int_{0}^{t} e^{(t-s) L}\left(-\nabla \cdot\left(\rho_{ \pm} u_{ \pm}\right)\right) d s\right| \\
\leq \sup _{0 \leq s \leq t} \int_{0}^{t} e^{(t-s) L}\left|\nabla \cdot\left(\rho_{ \pm} u_{ \pm}\right)\right| d s \\
\leq C \varepsilon_{13}\left(V_{0}\right)(1+t)^{-2} \\
\left\|g_{2}(t)\right\|_{L^{\infty}}=\sup _{0 \leq s \leq t}\left|\int_{0}^{t} e^{(t-s) L}\left(-u_{ \pm} \cdot \nabla u_{ \pm}-A_{ \pm} \gamma\left[\left(\rho_{ \pm}+1\right)^{\gamma-2}-1\right] \nabla \rho_{ \pm} \pm\left(u_{ \pm} \times B\right)+\alpha_{ \pm}\left(u_{ \pm}-u_{\mp}\right)\right) d s\right| \\
\leq \sup _{0 \leq s \leq t} \int_{0}^{t} e^{(t-s) L}\left|-u_{ \pm} \cdot \nabla u_{ \pm}-A_{ \pm} \gamma\left[\left(\rho_{ \pm}+1\right)^{\gamma-2}-1\right] \nabla \rho_{ \pm} \pm\left(u_{ \pm} \times B\right)+\alpha_{ \pm}\left(u_{ \pm}-u_{\mp}\right)\right| d s \\
\leq C \varepsilon_{13}\left(V_{0}\right)(1+t)^{-2}, \\
\left\|g_{3}(t)\right\|_{L^{\infty}}=\sup _{0 \leq s \leq t}\left|\int_{0}^{t} e^{(t-s) L}\left(\rho_{-} u_{-}-\rho_{+} u_{+}\right) d s\right| \\
\leq \sup _{0 \leq s \leq t} \int_{0}^{t} e^{(t-s) L}\left|\rho_{-} u_{-}-\rho_{+} u_{+}\right| d s \\
\leq C \varepsilon_{13}\left(V_{0}\right)(1+t)^{-2} .
\end{gathered}
$$

Thus,

$$
\left\|U-e^{t L} U_{0}\right\|_{L^{\infty}} \leq C \varepsilon_{13}\left(V_{0}\right)(1+t)^{-2} .
$$

So, by $L^{2}-L^{\infty}$ interpolation,

$$
\left\|U-e^{t L} U_{0}\right\|_{L^{q}} \leq C \varepsilon_{13}\left(V_{0}\right)(1+t)^{-2+\frac{3}{2 q}},
$$

for $2 \leq q \leq \infty$. This completes the proof of Theorem 1.1. 


\section{Part II}

\section{Heteroclinic Orbits For The nonlinear Vlasov And The one-dimensional Vlasov-Poisson Systems}




\section{Chapter 5}

\section{Introduction}

\subsection{Overview}

An important problem in Dynamical Systems is the existence of heteroclinic orbits, i.e. orbits that are asymptotically connecting two distinct critical points or manifolds. There is a rich literature in the case of planar systems, and here we recommend the survey paper [24] by Feng and $\mathrm{Hu}$, which reviews various methods for proving existence of or constructing both homoclinic and heteroclinic orbits. As expected, the dynamics is more complicated in higher dimensions, and thus much less is known in this case. About three decades ago, Rabinowitz [33] used a natural variational approach to prove existence of heteroclinic orbits for

$$
\ddot{q}(t)=-\nabla W(q(t)), \quad t \in \mathbb{R},
$$

where $W \in C^{2}\left(\mathbb{T}^{d}\right)$ and satisfies the assumptions (5.3) below. The idea is to fix $\xi \in \mathbb{Z}^{d}$ and look for minimizers of

$$
I[u]:=\frac{1}{2} \int_{\mathbb{R}}\left[|\dot{u}(t)|^{2}-W(u(t))\right] d t
$$

among all sufficiently regular curves $u: \mathbb{R} \rightarrow \mathbb{R}^{d}$ which stay sufficiently far from $\mathbb{Z}^{d} \backslash\{\xi\}$ and satisfy

$$
u(-\infty):=\lim _{t \rightarrow-\infty} u(t)=0 \in \mathbb{R}^{d} \text { and } u(\infty):=\lim _{t \rightarrow \infty} u(t)=\xi .
$$

This variational approach has been used by other authors, in different situations (multiple global minima, double-well potentials etc): [16], [19], [35] etc. Similar constructions have been employed to prove existence of heteroclinic orbits for PDE, or, 
in general, to prove the existence of connections between critical points in infinitedimensional metric spaces; see, for example, [30], [31], [36].

In recent years it has been revealed that some important evolutionary PDE arising in Atmospheric Science, Pressureless Gas Dynamics, Plasma Physics, etc. can be interpreted as infinite-dimensional Hamiltonian dynamical systems in spaces of measures. The dynamical viewpoint (where the time-continuous deformation of one distribution into the other is tracked) of the optimal (with respect to some given cost) transfer between two Borel measures of equal mass was pioneered by Benamou and Brenier [22] and has led to the discovery of many important connections between the Monge-Kantorovich theory and Fluid Dynamics. The set $\mathscr{P}_{2}\left(\mathbb{R}^{d}\right.$ ) (of Borel probabilities on $\mathbb{R}^{d}$ with finite second moment) equipped with the quadratic Monge-Kantorovich metric (see below) can be endowed with a Riemannian-like structure; Otto [32] used the geometric insight (later made rigorous by Ambrosio, Gigli and Savaré [18]) gained this way to prove useful asymptotic decay estimates for porous media. In [22] it is shown that the Euler-Lagrange equation satisfied by the geodesics of this "pseudo-Riemannian" metric space is the pressureless Euler system. An earlier connection between equations of Fluid Dynamics and infinitedimensional Geometry, not unrelated to Benamou and Brenier's [22], is the work of Arnold [20], which, in the words of Schnirelman [34], marked the beginning of "serious interaction between the dynamics of an ideal incompressible fluid and the (infinite-dimensional) differential geometry".

The realization that some classical evolutionary PDE (e.g. the Fokker-Planck equation) are gradient flows in such metric spaces has had a profound impact on the understanding of the mechanisms driving these flows. For example, whereas it had been long known that the negative Boltzmann entropy was an $\mathrm{H}$-functional (counterpart of a Lyapunov function for ODE) for the Heat Equation, Jordan, Kinderlehrer and Otto [28] showed that this equation is, in fact, the gradient flow of the entropy in the non-Euclidean setting provided by $\mathscr{P}_{2}\left(\mathbb{R}^{d}\right)$. Soon following it was realized that, beside these dissipative equations, there are systems of PDE (such as pressureless Euler-Poisson, nonlinear Vlasov, or the Semi-Geostrophic equations) that are conservative in the same setting. As opposed to the gradient flow case, the solutions to these conservative systems exhibit strong singularities and can be singular measures (i.e. not absolutely continuous with respect to the Lebesgue measure). This adds to the difficulty of the analysis, and reinforces our choice of setting the problem in spaces of measures.

The requirement $W \in C^{2}\left(\mathbb{T}^{d}\right)$ is sufficient to ensure existence and uniqueness of solutions for the nonlinear Vlasov system

$$
\partial_{t} f+v \cdot \nabla_{x} f=\nabla_{v} \cdot[f(\nabla W * \rho)], \text { where } \rho(t, x):=\int_{\mathbb{R}^{d}} f(t, x, v) d v,
$$

when $f_{0}:=f(0, \cdot, \cdot)$ is prescribed. It models the kinetics of a system of charged particles which interact according to a potential $W$ (see [37], which also covers the 
interpretation of this system as a Hamiltonian system in the space of probability measures endowed with a certain Monge-Kantorovich metric).

Definition 1. We say that $[0, T] \ni t \rightarrow f_{t}(d x, d v) \in \mathscr{P}\left(\mathbb{R}^{d} \times \mathbb{R}^{d}\right)$ is a weak solution to $(\mathrm{NV})$ on $\mathbb{R}^{d} \times \mathbb{R}^{d}$ if

$$
[0, T] \ni t \rightarrow \int_{\mathbb{R}^{d}} \int_{\mathbb{R}^{d}} \varphi(x, v) f_{t}(d x, d v) \in W^{1,1}(0, T)
$$

and

$$
\begin{aligned}
\frac{d}{d t} \int_{\mathbb{R}^{d}} \int_{\mathbb{R}^{d}} \varphi(x, v) f_{t}(d x, d v) & =\int_{\mathbb{R}^{d}} \int_{\mathbb{R}^{d}} v \cdot \nabla_{x} \varphi(x, v) f_{t}(d x, d v) \\
& -\int_{\mathbb{R}^{d}} \int_{\mathbb{R}^{d}} \nabla W * \rho_{t}(x) \cdot \nabla_{\nu} \varphi(x, v) f_{t}(d x, d v)
\end{aligned}
$$

for any $\varphi \in C_{c}\left(\mathbb{R}^{d} \times \mathbb{R}^{d}\right)$, where $\rho_{t}$ denotes the $x$-marginal of $f_{t}$.

We point the reader to [17] and [29] for issues like existence, uniqueness and stability properties for the nonlinear Vlasov equation. We add three assumptions on $W$, i.e.

$$
W(-x)=W(x)<W(0)=0 \text { for all } x \in \mathbb{R}^{d} \backslash \mathbb{Z}^{d} .
$$

Let $n \geq 1$ be an integer and define $L^{n}: \mathbb{R}^{d \times n} \times \mathbb{R}^{d \times n} \rightarrow \mathbb{R}$ by

$$
L^{n}(X, V)=\frac{1}{2}|V|_{n}^{2}-\frac{1}{2 n^{2}} \sum_{i, j=1}^{n} W\left(x_{i}-x_{j}\right)
$$

where $|\cdot|_{n}$ is the norm on $\mathbb{R}^{d \times n}$ induced by the inner-product

$$
\langle X, Y\rangle_{n}:=\frac{1}{n} \sum_{j=1}^{n} x_{j} \cdot y_{j}
$$

(the dot represents the usual dot product in $\mathbb{R}^{d}$ ). The Euler-Lagrange equations associated to $L^{n}$ are

$$
\ddot{x}_{i}(t)=-\frac{1}{n} \sum_{j=1}^{n} \nabla W\left(x_{i}(t)-x_{j}(t)\right), \quad i=1, \ldots, n .
$$

For all $t \in \mathbb{R}$ let

$$
f^{n}(t, x, v):=\frac{1}{n} \sum_{i=1}^{n} \delta_{\left(x_{i}(t), \dot{x}_{i}(t)\right)}(x, v)\left(\text { so its } x \text {-marginal is } \rho^{n}(t, x)=\frac{1}{n} \sum_{j=1}^{n} \delta_{x_{j}(t)}(x)\right) .
$$

The connection between the system (5.5) and (NV) has first been explored in [25] in the context of action-minimizing monokinetic solutions to (NV) and is the result of the following observation: 
Proposition 5.1. If $X^{n}=\left[x_{1}, \ldots, x_{n}\right]$ solves (5.5), then $f^{n}$ solves $(\mathrm{NV})$ in the sense of distributions.

Proof: Let $\varphi \in C_{c}^{1}\left(\mathbb{R}^{d} \times \mathbb{R}^{d}\right)$ and use (5.6) to compute:

$\frac{d}{d t} \int_{\mathbb{R}^{d}} \int_{\mathbb{R}^{d}} \varphi(x, v) f^{n}(t, d x, d v)=\frac{1}{n} \sum_{j=1}^{n}\left[\dot{x}_{j}(t) \cdot \nabla_{x} \varphi\left(x_{j}(t), \dot{x}_{j}(t)\right)+\ddot{x}_{j}(t) \cdot \nabla_{v} \varphi\left(x_{j}(t), \dot{x}_{j}(t)\right)\right]$.

We use (5.5) and (5.6) again to recognize the right hand side of the equation above as

$$
\int_{\mathbb{R}^{d}} \int_{\mathbb{R}^{d}}\left\{v \cdot \nabla_{x} \varphi(x, v)-\left[\nabla W * \rho^{n}(t, \cdot)\right](x) \cdot \nabla_{v} \varphi(x, v)\right\} f^{n}(t, d x, d v),
$$

which finishes the proof.

QED.

In [33], P. Rabinowitz proved the following theorem (slightly reworded here):

Theorem 5.1. Let $\mathscr{V} \in C^{2}\left(\mathbb{T}^{m}\right)$ satisfy $\mathscr{V}(-x)=\mathscr{V}(x)<\mathscr{V}(0)=0$ for all $x \in \mathbb{R}^{m} \backslash \mathbb{Z}^{m}$ (note that this implies that the absolute maximum points of $\mathscr{V}$ are isolated). Then, for any $z \in \mathbb{Z}^{m}$ there exist two solutions $q^{\mp}: \mathbb{R} \rightarrow \mathbb{R}^{m}$ of $\ddot{q}=-\nabla \mathscr{V}(q)$ such that $q^{\mp}(\mp \infty)=z$ and $q^{\mp}( \pm \infty) \in \mathbb{Z}^{m} \backslash\{z\}$.

Put in words, this means that for any $z \in \mathbb{Z}$ there exists a heteroclinic orbit for the system $\ddot{q}=-\nabla \mathscr{V}(q)$ which emanates from $z$ and another one which terminates at $z$. Of course, it is easy to notice that given $q^{-}, q^{+}$can be chosen such that $q^{+}(t):=$ $q^{-}(-t)$.

We denote

$$
\mathscr{W}^{n}(X):=\frac{1}{2 n^{2}} \sum_{i, j=1}^{n} W\left(x_{i}-x_{j}\right),
$$

which achieves its global maximum only at all matrices of the form

$$
X=\left[x, x+z_{1}, \ldots, x+z_{n-1}\right] \text { for all } x \in \mathbb{R}^{d} \text { and all } z_{i} \in \mathbb{Z}^{d}, i=1, \ldots, n-1 .
$$

For a given $Z:=\left[z_{1}, \ldots, z_{n-1}\right] \in \mathbb{Z}^{d \times(n-1)}$ let

$$
\mathscr{M}(Z):=\left\{X \in \mathbb{R}^{d \times n}: X=\left[x, x+z_{1}, \ldots, x+z_{n-1}\right], x \in \mathbb{R}^{d}\right\}
$$

and let

$$
\mathscr{M}:=\bigcup_{Z \in \mathbb{R}^{d \times(n-1)}} \mathscr{M}(Z) .
$$

Since $\mathscr{M} \subset \operatorname{Argmax} \mathscr{W}^{n}$ and none of the points in $\mathscr{M}$ is isolated, Rabinowitz's results will not apply directly to (5.5) to provide existence of heteroclinic orbits emanating from and terminating at every point in $\operatorname{Argmax} \mathscr{W}^{n}$. However, it is transparent that upon setting

$$
Y:=\left[y_{1}, \ldots, y_{n-1}\right] \text { for } y_{i}:=x_{1}-x_{i+1}, i=1, \ldots, n-1,
$$


we get that $Y: \mathbb{R} \rightarrow \mathbb{R}^{d \times(n-1)}$ solves

$$
\ddot{y}_{i}=-\frac{2}{n} \nabla W\left(y_{i}\right)-\frac{1}{n} \sum_{j \neq i}^{n-1}\left[\nabla W\left(y_{j}\right)+\nabla W\left(y_{i}-y_{j}\right)\right], \quad i=1, \ldots, n-1 .
$$

One can then recover $X$ from $Y$ up to an affine function of $t$ by first integrating

$$
\ddot{x}_{1}=-\frac{1}{n} \sum_{i=1}^{n-1} \nabla W\left(y_{i}\right)
$$

and then setting

$$
x_{i}:=x_{1}-y_{i-1} \text { for } i=2, \ldots, n .
$$

More precisely, $X$ solves (5.5) if and only if $Y$, as defined in (5.10), solves (5.11) and (5.12) is satisfied. This affine transformation from $X \in \mathbb{R}^{d \times n}$ to $Y \in \mathbb{R}^{d \times(n-1)}$ turns out to be very helpful, as the expression in the right hand side of (5.11) is still a gradient (with respect to an appropriately chosen inner-product; see Proposition 8.1 in Appendix). Clearly, $\tilde{\mathscr{W}}^{n}$ satisfies (5.3) over $\mathbb{R}^{d \times(n-1)}$ (which implies the maximum points are isolated, since they form the set $\left.\mathbb{Z}^{d \times(n-1)}\right)$. A careful inspection of the proof of Theorem 5.1 in [33] reveals that it holds true regardless of which innerproduct $\mathbb{R}^{m}$ is equipped with. Thus, by taking $m=d \times(n-1)$ and $\mathscr{V}=\tilde{\mathscr{W}}^{n}$, we deduce that for any $Z \in \mathbb{Z}^{d \times(n-1)}$ there exist two solutions $Y^{\mp}: \mathbb{R} \rightarrow \mathbb{R}^{d \times(n-1)}$ for (5.11) such that $Y^{\mp}(\mp \infty)=Z$ and $Y^{\mp}( \pm \infty) \in \mathbb{Z}^{d \times(n-1)} \backslash\{Z\}$. Reverting from $Y$ to $X$ as indicated above, we translate the above result into:

Proposition 5.2. For any $Z \in \mathbb{Z}^{d \times(n-1)}$ there exist two solutions $X^{\mp}: \mathbb{R} \rightarrow \mathbb{R}^{d \times n}$ for (5.5) such that

$$
\lim _{t \rightarrow \mp \infty} \operatorname{dist}\left(X^{\mp}(t), \mathscr{M}(Z)\right)=0 \text { and } \lim _{t \rightarrow \pm \infty} \operatorname{dist}\left(X^{\mp}(t), \mathscr{M} \backslash \mathscr{M}(Z)\right)=0,
$$

where for a metric space $(S, d)$ and any $s \in S$ and $A \subset S$, we have $\operatorname{dist}(s, A):=$ $\inf _{a \in A} d(s, a)$.

\subsection{Main results}

One can write [25] a Lagrangian which coincides with $L^{n}$ when restricted to averages of $n$ Dirac masses. It can be defined on

$$
\bigcup_{\mu \in \mathscr{P}_{2}\left(\mathbb{R}^{d}\right)}\left(\{\mu\} \times L^{2}\left(\mu ; \mathbb{R}^{d}\right)\right)
$$

by

$L(\mu, \zeta)=\frac{1}{2}\|\zeta\|_{L^{2}\left(\mu ; \mathbb{R}^{d}\right)}^{2}-\frac{1}{2} \int_{\mathbb{R}^{d}} \int_{\mathbb{R}^{d}} W(x-y) \mu(d x) \mu(d y)=: \frac{1}{2}\|\zeta\|_{L^{2}\left(\mu ; \mathbb{R}^{d}\right)}^{2}-\frac{1}{2} \mathscr{W}(\mu)$.

It is not difficult to see that the "continuous version" of $\mathscr{W}^{n}$ is $\mathscr{W}$. 
Remark 5.1. It is proved in [25] that the monokinetic solutions of (NV) correspond to the Euler-Lagrange equation associated to the Lagrangian (5.14). The same reference shows that the fully kinetic version of (NV) (i.e. (NV) itself) is the EulerLagrange equation associated to a related Lagrangian defined on $\mathscr{P}_{2}\left(\mathbb{R}^{d} \times \mathbb{R}^{d}\right)$, in the sense specified in [17]. We do not need to discuss the details here, so we refer the reader to [17] and [25] for further details.

If

$$
\Lambda:=\left\{\lambda:=\left(\lambda_{z}\right)_{z \in \mathbb{Z}^{d}}: \lambda_{z} \geq 0 \text { for } z \in \mathbb{Z}^{d} \text { and } \sum_{z \in \mathbb{Z}^{d}} \lambda_{z}=1\right\}
$$

let

$$
f_{x, \lambda}^{\infty}:=\sum_{z \in \mathbb{Z}^{d}} \lambda_{z} \delta_{(x+z, 0)} \text { for some } \lambda \in \Lambda, x \in \mathbb{R}^{d}
$$

Let us denote

$$
\mathscr{F}_{\lambda}:=\left\{f_{x, \lambda}^{\infty}: x \in \mathbb{R}^{d}\right\} \text { if } \lambda \in \Lambda, \text { and } \mathscr{F}:=\bigcup_{\lambda \in \Lambda} \mathscr{F}_{\lambda} .
$$

We shall prove first that $\mathscr{F}$ is the set of all critical points (measures, in our context) of (NV) whose $x$-marginals maximize the potential $\mathscr{W}$. For critical measures whose $x$-marginals are not maximizers of $\mathscr{W}$ we do not expect such heteroclinic orbits to exist in general; this is consistent with the finite dimensional case of mechanical Hamiltonians $(1 / 2)|p|^{2}+F(x)$, where critical points associated to minima of $F$ are, in general, stable.

Remark 5.2. Note that, in light of Proposition 5.1, Proposition 5.2 provides heteroclinic orbits for (NV) originating and terminating at measures of the form

$$
\bar{f}^{n}:=\frac{1}{n} \sum_{j=1}^{n} \delta_{\left(x+z_{j}, 0\right)} \in \mathscr{P}_{2}\left(\mathbb{R}^{d} \times \mathbb{R}^{d}\right) \text {, where } x \in \mathbb{R}^{d}, z_{j} \in \mathbb{Z}^{d}, j=1, \ldots, n \text {. }
$$

This result is, however, too restrictive, as it only covers a subset of critical points whose $x$-marginals are maximizers of the potential $\mathscr{W}$.

We hope that by now it is apparent that our goal is to formulate and prove a result of the type Proposition 5.2 for (NV), i.e. we would like to establish the existence of heteroclinic orbits emanating and terminating at $\mathscr{F} \lambda$ for any $\lambda \in \Lambda$. We will prove the following (in fact, a more precise version will be presented in the next Chapter; see Theorem 6.1 and Corollary 6.1):

Theorem 5.2. Let $\lambda \in \Lambda$. There exist two solutions $f^{\mp}: \mathbb{R} \rightarrow \mathscr{P}_{2}\left(\mathbb{R}^{d} \times \mathbb{R}^{d}\right)$ of $(\mathrm{NV})$ such that

$$
\lim _{t \rightarrow \mp \infty} \operatorname{dist}_{W_{2}}\left(f^{\mp}, \mathscr{F}_{\lambda}\right)=0 \text { and } \lim _{t \rightarrow \pm \infty} \operatorname{dist}_{W_{2}}\left(f^{\mp}, \mathscr{F} \backslash \mathscr{F}_{\lambda}\right)=0,
$$

where dist $_{W_{2}}$ is defined with respect to the Wasserstein distance $W_{2}$ on $\mathscr{P}_{2}\left(\mathbb{R}^{d} \times \mathbb{R}^{d}\right)$. 
We define the Wasserstein distance as (see, e.g., [37]):

Definition 2. If $\mu, v$ are Borel probability measures in $\mathbb{R}^{m}$, then

$$
W_{2}(\mu, v):=\left(\inf _{\gamma \in \Gamma(\mu, v)} \int_{\mathbb{R}^{m}} \int_{\mathbb{R}^{m}}|x-y|^{2} \gamma(d x, d y)\right)^{\frac{1}{2}},
$$

where

$$
\begin{aligned}
\Gamma(\mu, v):= & \left\{\gamma \in \mathscr{P}\left(\mathbb{R}^{m} \times \mathbb{R}^{m}\right): \int_{\mathbb{R}^{m}} \int_{\mathbb{R}^{m}} \varphi(x) \gamma(d x, d y)=\int_{\mathbb{R}^{m}} \varphi(x) \mu(d x),\right. \\
& \left.\int_{\mathbb{R}^{m}} \int_{\mathbb{R}^{m}} \varphi(y) \gamma(d x, d y)=\int_{\mathbb{R}^{m}} \varphi(y) \nu(d y) \text { for all } \varphi \in C_{b}\left(\mathbb{R}^{m}\right)\right\} .
\end{aligned}
$$

In Chapter 3 of this paper we analyze a singular version of (NV), namely the Vlasov-Poisson system. Here we restrict ourselves to $d=1$ and show that the initial value problem associated with a version of the periodic, one-dimensional VlasovPoisson system that takes into account the possibility of measure-valued solutions (see [27] for the Euclidean, non-periodic case) is ill-posed. Furthermore, we explicitly construct infinitely many non-trivial periodic solutions, as well as infinitely many homoclinic and heteroclinic orbits for said system. The following conclusions will follow from that analysis:

Theorem 5.3. (1) The periodic Vlasov-Poisson equation exhibits nonuniqueness of distributional solutions for the initial-value problem.

(2) The periodic Vlasov-Poisson equation admits infinitely many periodic (nontrival) distributional solutions.

(3) There exist infinitely many homoclinic orbits associated with the steady state $\delta_{(0,0)}$.

(4) There exist infinitely many heteroclinic orbits originating at $\boldsymbol{\delta}_{(0,0)}$ and infinitely many terminating at $\boldsymbol{\delta}_{(0,0)}$.

We conclude with an Appendix Chapter where we mainly visit or re-visit some concepts and results that are useful throughout Chapter 3 and are more or less known in the literature. 


\section{Chapter 6}

\section{Heteroclinic orbits for the Nonlinear Vlasov system}

As customary, we define $\mathbb{T}^{d}:=\mathbb{R}^{d} / \mathbb{Z}^{d}$,

$$
C\left(\mathbb{T}^{d}\right):=\left\{f: \mathbb{R}^{d} \rightarrow \mathbb{R}: f \text { is continuous and } \mathbb{Z}^{d} \text {-periodic }\right\} .
$$

We also use

$$
|x|_{\mathbb{T}^{d}}:=\min _{k \in \mathbb{Z}^{d}}|x+k|,
$$

so that $d(x, y):=|x-y|_{\mathbb{T}^{d}}$ defines a metric on $\mathbb{T}^{d}$. We begin with the following definition:

\section{Definition 3.}

1. We say that $\mu, v \in \mathscr{P}\left(\mathbb{R}^{d}\right)$ (the set of all Borel probabilities on $\mathbb{R}^{d}$ ) are $\mathbb{T}^{d}$ equivalent if

$$
\int_{\mathbb{R}^{d}} \varphi d \mu=\int_{\mathbb{R}^{d}} \varphi d \nu \text { for all } \varphi \in C\left(\mathbb{T}^{d}\right)
$$

2. If the above holds, we write $\mu \sim v$.

\section{Remark 6.1.}

1. It is known [26] that “ " is an equivalence relation on $\mathscr{P}\left(\mathbb{R}^{d}\right)$. We denote

$$
\mathscr{P}\left(\mathbb{R}^{d}\right) / \sim \text { by } \mathscr{P}\left(\mathbb{T}^{d}\right) .
$$

2. It is also known [26] that each equivalence class (or each $\mathscr{P}\left(\mathbb{T}^{d}\right)$ - measure) admits a unique representative $\mu \in \mathscr{P}\left(Q_{d}\right)$, where $Q_{d}:=[0,1)^{d}$. We provide more details in Chapter 8.2.

3. One can prove that given any $\mu \in \mathscr{P}\left(\mathbb{R}^{d}\right)$ there exists a unique $\mu^{*} \in \mathscr{P}\left(Q_{d}\right)$ such that $\mu^{*} \sim \mu$. The uniqueness is equivalent to:

$$
\mu \sim v \text { if and only if } \mu^{*}=v^{*} .
$$


Indeed, assume $\theta, \vartheta \in \mathscr{P}\left(\mathbb{R}^{d}\right)$ such that $\theta\left(Q_{d}\right)=\vartheta\left(Q_{d}\right)=1$ and

$$
\int_{\mathbb{R}^{d}} \varphi d \theta=\int_{\mathbb{R}^{d}} \varphi d \vartheta \text { for all } \varphi \in C\left(\mathbb{T}^{d}\right) .
$$

Let $\zeta \in C_{b}\left(\mathbb{R}^{d}\right)$ be arbitrarily fixed. There exists a sequence $\left\{\varphi_{n}\right\}_{n} \subset C\left(\mathbb{T}^{d}\right)$ bounded in the sup norm and pointwise convergent everywhere in $Q_{d}$ to $\left.\zeta\right|_{Q_{d}}$. (One way to construct such sequence would be by first multiplying $\zeta$ by a continuous function $u_{n}$ which is equal to 1 on the cube $[0,1-1 / n]^{d}$ and 0 on $\Omega:=Q_{d} \backslash[0,1-1 /(2 n)]^{d}$. Then one can solve a Laplace boundary value problem in $\Omega$ by prescribing the boundary value 0 on the portion of the boundary inside $Q_{d}$ and appropriate values elsewhere such that the corresponding values on opposite faces of $Q_{d}$ match the values of $\zeta u_{n}$. The functions obtained by patching these solutions and $\zeta u_{n}$ are in $C\left(\mathbb{T}^{d}\right)$, converge pointwise everywhere to $\zeta$ in $Q_{d}$ and, as a consequence of the maximum principle for the Laplace equation, are all bounded in the sup norm by sup $|\zeta|$.) By dominated convergence we conclude

$$
\int_{\mathbb{R}^{d}} \zeta d \theta=\int_{\mathbb{R}^{d}} \zeta d \vartheta \text { for all } \zeta \in C_{b}\left(\mathbb{R}^{d}\right)
$$

so $\theta=\vartheta$.

The proposition below fully characterizes the maximizers of the potential energy.

Proposition 6.1. Under the hypotheses (5.3) on $W$ we have that the only maximizers of the potential energy

$$
I(\mu):=\frac{1}{2} \int_{\mathbb{R}^{d}} \int_{\mathbb{R}^{d}} W(x-y) \mu(d y) \mu(d x)
$$

in each equivalence class are the Dirac measures $\delta_{a}$, where $a \in Q_{d}$ (i.e. if $\mu \in$ $\mathscr{P}\left(\mathbb{R}^{d}\right)$ is a maximizer, then $\mu^{*}=\delta_{a}$ for some $\left.a \in Q_{d}\right)$.

Proof: Since $W \in C\left(\mathbb{T}^{d}\right)$, we see that $I$ is invariant in each equivalence class. In particular, $I(\mu)=I\left(\mu^{*}\right)$ for all $\mu \in \mathscr{P}_{2}\left(\mathbb{R}^{d}\right)$. Since $I\left(\delta_{a}\right)=0 \geq I\left(\mu^{*}\right)$ for all $\mu^{*} \in$ $\mathscr{P}\left(Q_{d}\right)$ and for all $a \in Q_{d}$, we deduce that the global maximum of $I$ is 0 and it is achieved at $\delta_{a}$. Now, if we look for $\mu^{*} \in \mathscr{P}\left(Q_{d}\right)$ such that $I\left(\mu^{*}\right)=0$, we see from (5.3) that $\mu^{*} \otimes \mu^{*}$ must be supported on the diagonal of $Q_{d} \times Q_{d}$. Thus, for any Borel sets $A, B \subset Q_{d}$ such that $A \cap B=\emptyset$ we have $0=\left(\mu^{*} \otimes \mu^{*}\right)(A \times B)=\mu^{*}(A) \mu^{*}(B)$, i.e $\mu^{*}(A)=0$ or $\mu^{*}(B)=0$. We claim that this implies the existence of $a \in Q_{d}$ such that $\mu^{*}=\delta_{a}$. Indeed, let us divide the cube $Q_{d}$ into $2^{d}$ equal, disjoint subcubes, i.e.

$$
Q_{d}=\bigcup_{j=1}^{2^{d}} Q_{d}^{j}, \text { where } Q_{d}^{j}:=\left[a_{1}^{j}, a_{1}^{j}+1\right) \times\left[a_{2}^{j}, a_{2}^{j}+1\right) \times \ldots \times\left[a_{d}^{j}, a_{d}^{j}+1\right) \text {. }
$$

Since $\mu^{*}\left(Q_{d}\right)=1$, according to the previous observations, there exists $j_{0} \in\left\{1, \ldots, 2^{d}\right\}$ such that $\mu^{*}\left(Q_{d}^{j_{0}}\right)=1$ and $\mu^{*}\left(Q_{d}^{j}\right)=0$ if $j \neq j_{0}$. Next we divide $Q_{d}^{j_{0}}$ into $2^{d}$ equal, 
disjoint subcubes etc. Thus, we obtain a sequence of Borel sets $A_{1} \supset A_{2} \supset \ldots \supset A_{k} \supset$ $\ldots$, such that $\mu^{*}\left(A_{k}\right)=1$ for all $k \geq 1$, which implies

$$
\mu^{*}\left(\bigcap_{k \geq 1} A_{k}\right)=1 .
$$

Since the sets $A_{k}$ are cubes with $\lim _{k \rightarrow \infty} \mathscr{L}^{d}\left(A_{k}\right)=0$, we infer that $\bigcap_{k \geq 1} A_{k}$ is a singleton. QED.

\section{Remark 6.2.}

1. Thus, any measure $\mu \in \mathscr{P}_{2}\left(\mathbb{R}^{d}\right)$ that maximizes $I$ is $\mathbb{T}^{d}$-equivalent to $\delta_{a}$ for some $a \in Q_{d}$. It is not difficult to see that this is equivalent to maximizers $\mu$ being of the form:

$$
\mu=\sum_{z \in \mathbb{Z}^{d}} \lambda_{z} \delta_{a+z}
$$

where $a \in Q_{d}$ and $\lambda_{z} \geq 0$ such that $\sum_{z \in \mathbb{Z}^{d}} \lambda_{z}=1$.

2. We will prove in this Chapter that for any $\mu$ of the form (6.1) above there exists a heteroclinic orbit

$$
\mathbb{R} \ni t \rightarrow f(t) \in \mathscr{P}_{2}\left(\mathbb{R}^{d} \times \mathbb{R}^{d}\right)
$$

such that

$$
\lim _{t \rightarrow-\infty} f(t)=\sum_{z \in \mathbb{Z}^{d}} \lambda_{z} \delta_{(a+z, 0)} \text { and } \lim _{t \rightarrow \infty} f(t)=v,
$$

where $v$ is another maximizer which is not in the same equivalence class as $f(-\infty)$. 3. In fact, we shall see that there exist a continuum of measures $v$ as in 2.

The next proposition provides the main constructive idea for our most general result (see Theorem 6.1 and its corollary). It is, basically, a simpler, particular case. The reader may skip it and continue directly to Theorem 6.1.

Proposition 6.2. Let $\xi \in \mathbb{Z}^{d} \backslash\{0\}$ and let $q: \mathbb{R} \rightarrow \mathbb{R}^{d}$ be a solution of $\ddot{q}=-\nabla W(q)$ such that $q(-\infty)=0$ and $q(\infty)=\xi$ (this is guaranteed to exist for some $\xi \in \mathbb{Z}^{d} \backslash\{0\}$ by Theorem 5.1). Fix $\alpha \in[0,1]$. Then

$$
f_{\alpha}(t):=\alpha \delta_{((\alpha-1) q(t),(\alpha-1) \dot{q}(t))}+(1-\alpha) \delta_{(\alpha q(t), \alpha \dot{q}(t))}
$$

solves the (NV) system. Furthermore,

$$
f_{\alpha}(-\infty)=\delta_{(0,0)}
$$

and

$$
f_{\alpha}(\infty):=\alpha \delta_{((\alpha-1) \xi, 0)}+(1-\alpha) \delta_{(\alpha \xi, 0)}
$$




\section{Remark 6.3.}

1. Note that, indeed, $f_{\alpha}(\infty)$ is a stationary solution for $(\mathrm{NV})$.

Proof : Since

$$
\nabla W * \rho(x)=\int_{\mathbb{R}} \nabla W(x-y) \rho(d y) \text { and } \rho=\alpha \delta_{(\alpha-1) \xi}+(1-\alpha) \delta_{\alpha \xi}
$$

we get

$$
\begin{aligned}
& \int_{\mathbb{R}^{d}} \int_{\mathbb{R}^{d}}\left\{-v \cdot \nabla_{x} \varphi(x, v)+[\nabla W * \rho(x)] \cdot \nabla_{\nu} \varphi(x, v)\right\} f_{\infty}(d x, d v) \\
= & \int_{\mathbb{R}} \int_{\mathbb{R}}[\alpha \nabla W(x-(\alpha-1) \xi)+(1-\alpha) \nabla W(x-\alpha \xi)] \cdot \nabla_{v} \varphi(x, v) f_{\infty}(d x, d v) \\
= & \alpha[\alpha \nabla W((\alpha-1) \xi-(\alpha-1) \xi)+(1-\alpha) \nabla W((\alpha-1) \xi-\alpha \xi)] \cdot \nabla_{\nu} \varphi((\alpha-1) \xi, 0) \\
+ & (\alpha-1)[\alpha \nabla W(\alpha \xi-(\alpha-1) \xi)+(1-\alpha) \nabla W(\alpha \xi-\alpha \xi)] \cdot \nabla_{\nu} \varphi(\alpha \xi, 0)=0 .
\end{aligned}
$$

2. There exists a set $\mathscr{A} \subset[0,1]$ with cardinality $c$ (cardinality of $\mathbb{R}$ ) such that $f_{\alpha_{1}}(\infty)$ is not $\mathbb{T}^{d}$-equivalent to $f_{\alpha_{2}}(\infty)$ for all $\alpha_{1} \neq \alpha_{2} \in \mathscr{A}$.

Proof (of Proposition 6.2) : To check that $f_{\alpha}$ solves (NV) we take $\varphi \in C_{c}^{1}\left(\mathbb{R}^{d} \times \mathbb{R}^{d}\right.$ ) and see that:

$$
\begin{aligned}
& \frac{d}{d t} \int_{\mathbb{R}^{d}} \int_{\mathbb{R}^{d}} \varphi(x, v) f_{\alpha}(t, d x, d v) \\
& =\frac{d}{d t}[\alpha \varphi((\alpha-1) q(t),(\alpha-1) \dot{q}(t))+(1-\alpha) \varphi(\alpha q(t), \alpha \dot{q}(t))] \\
& =\alpha(\alpha-1) \dot{q}(t) \cdot \nabla_{x} \varphi((\alpha-1) q(t),(\alpha-1) \dot{q}(t))+(1-\alpha) \alpha \dot{q}(t) \cdot \nabla_{x} \varphi(\alpha q(t), \alpha \dot{q}(t)) \\
& +\alpha(\alpha-1) \ddot{q}(t) \cdot \nabla_{v} \varphi((\alpha-1) q(t),(\alpha-1) \dot{q}(t))+(1-\alpha) \alpha \ddot{q}(t) \cdot \nabla_{\nu} \varphi(\alpha q(t), \alpha \dot{q}(t)) .
\end{aligned}
$$

The sum of the first two terms is

$$
\int_{\mathbb{R}^{d}} \int_{\mathbb{R}^{d}} v \cdot \nabla_{x} \varphi(x, v) f_{\alpha}(t, d x, d v) .
$$

We now use the $x$-marginal of $f_{\alpha}$, namely

$$
\rho_{\alpha}(t, \cdot)=\alpha \delta_{(\alpha-1) q(t)}+(1-\alpha) \delta_{\alpha q(t)},
$$

to see that

$$
\int_{\mathbb{R}^{d}} \nabla W(x-y) \rho_{\alpha}(t, d y)=\alpha \nabla W(x+(1-\alpha) q(t))+(1-\alpha) \nabla W(x-\alpha q(t)) .
$$


Thus,

$$
\begin{aligned}
\int_{\mathbb{R}^{d}} \int_{\mathbb{R}^{d}} & \left(\int_{R^{d}} \nabla W(x-y) \rho_{\alpha}(t, d y)\right) \cdot \nabla_{\nu} \varphi(x, v) f_{\alpha}(t, d x, d v) \\
= & \alpha^{2} \nabla W((\alpha-1) q(t)+(1-\alpha) q(t)) \cdot \nabla_{\nu} \varphi((\alpha-1) q(t),(\alpha-1) \dot{q}(t)) \\
& +\alpha(1-\alpha) \nabla W(\alpha q(t)+(1-\alpha) q(t)) \cdot \nabla_{\nu} \varphi(\alpha q(t), \alpha \dot{q}(t)) \\
& +\alpha(1-\alpha) \nabla W((\alpha-1) q(t)-\alpha q(t)) \cdot \nabla_{v} \varphi((\alpha-1) q(t),(\alpha-1) \dot{q}(t)) \\
& +(1-\alpha)^{2} \nabla W(\alpha q(t)-\alpha q(t)) \cdot \nabla_{\nu} \varphi(\alpha q(t), \alpha \dot{q}(t)) \\
= & \alpha(1-\alpha) \ddot{q}(t) \cdot \nabla_{v} \varphi((\alpha-1) q(t),(\alpha-1) \dot{q}(t))+\alpha(\alpha-1) \ddot{q}(t) \cdot \nabla_{v} \varphi(\alpha q(t), \alpha \dot{q}(t)) .
\end{aligned}
$$

Note that we used $\ddot{q}=-\nabla W(q)=\nabla W(-q)$ and $\nabla W(0)=0$. Thus we have proved:

$$
\begin{array}{r}
\frac{d}{d t} \int_{\mathbb{R}^{d}} \int_{\mathbb{R}^{d}} \varphi(x, v) f_{\alpha}(t, d x, d v)=\int_{\mathbb{R}^{d}} \int_{\mathbb{R}^{d}} v \cdot \nabla_{x} \varphi(x, v) f_{\alpha}(t, d x, d v) \\
-\int_{\mathbb{R}^{d}} \int_{\mathbb{R}^{d}}\left(\int_{\mathbb{R}^{d}} \nabla W(x-y) \rho_{\alpha}(t, d y)\right) \cdot \nabla_{\nu} \varphi(x, v) f_{\alpha}(t, d x, d v)
\end{array}
$$

for any $\varphi \in C_{c}^{1}\left(\mathbb{R}^{d} \times \mathbb{R}^{d}\right)$, i.e. $f_{\alpha}$ is a distributional solution for $(\mathrm{NV})$.

QED.

Despite (NV) being nonlinear in the term containing the convolution, the compatibility between the properties of the potential $W$ and the stationary solutions with maximizing potential allows us to put the building blocks from the previous proposition together in order to treat the most general case.

Theorem 6.1. Let $\alpha, \beta \in[0,1]$ such that $\alpha+\beta=1$ and $\lambda_{j}, \mu_{j} \in[0,1]$ such that

$$
\begin{array}{r}
\sum_{j=1}^{\infty} \lambda_{j}=\beta, \quad \sum_{j=1}^{\infty} \mu_{j}=\alpha . \text { Then for any } k_{j}, l_{j} \in \mathbb{Z}^{d}, \quad j=1, \ldots, \infty, \text { the measure } \\
f(t, \cdot, \cdot):=\sum_{j=1}^{\infty}\left(\lambda_{j} \delta_{\left(k_{j}+\alpha q(t), \alpha \dot{q}(t)\right)}+\mu_{j} \delta_{\left(l_{j}-\beta q(t),-\beta \dot{q}(t)\right)}\right)
\end{array}
$$

solves (NV) in the sense of distributions and satisfies

$$
f(-\infty)=\sum_{j=1}^{\infty}\left(\lambda_{j} \delta_{\left(k_{j}, 0\right)}+\mu_{j} \boldsymbol{\delta}_{\left(l_{j}, 0\right)}\right)
$$

and

$$
f(\infty)=\sum_{j=1}^{\infty}\left(\lambda_{j} \delta_{\left(k_{j}+\alpha \xi, 0\right)}+\mu_{j} \delta_{\left(l_{j}-\beta \xi, 0\right)}\right),
$$

which are both equilibrium solutions of (NV). 
Proof : Let

$$
\rho(t):=\sum_{j=1}^{\infty}\left(\lambda_{j} \delta_{k_{j}+\alpha q(t)}+\mu_{j} \delta_{l_{j}-\beta q(t)}\right)
$$

be the $x$-marginal of $f(t)$. Note that

$$
\begin{aligned}
\int_{\mathbb{R}^{d}} \nabla W(x-y) \rho(t, d y) & =\sum_{j=1}^{\infty}\left[\lambda_{j} \nabla W\left(x-k_{j}-\alpha q(t)\right)+\mu_{j} \nabla W\left(x-l_{j}+\beta q(t)\right)\right] \\
& =\beta \nabla W(x-\alpha q(t))+\alpha \nabla W(x+\beta q(t)) .
\end{aligned}
$$

Thus, we have

$$
\begin{aligned}
& \int_{\mathbb{R}^{d}} \int_{\mathbb{R}^{d}}\left(\int_{\mathbb{R}^{d}} \nabla W(x-y) \rho_{\alpha}(t) d y\right) \cdot \nabla_{\nu} \varphi(x, v) f_{\alpha}(t, d x, d v) \\
& =\beta \int_{\mathbb{R}^{d}} \int_{\mathbb{R}^{d}} \nabla W(x-\alpha q(t)) \cdot \nabla_{v} \varphi(x, v) f(t, d x, d v) \\
& +\alpha \int_{\mathbb{R}^{d}} \int_{\mathbb{R}^{d}} \nabla W(x+\beta q(t)) \cdot \nabla_{\nu} \varphi(x, v) f(t, d x, d v)=: \beta T_{1}+\alpha T_{2} .
\end{aligned}
$$

We compute

$$
\begin{aligned}
T_{1} & =\sum_{j=1}^{\infty} \lambda_{j} \nabla W\left(k_{j}+\alpha q(t)-\alpha q(t)\right) \cdot \nabla_{\nu} \varphi\left(k_{j}+\alpha q(t), \alpha \dot{q}(t)\right) \\
& +\sum_{j=1}^{\infty} \mu_{j} \nabla W\left(l_{j}-\beta q(t)-\alpha q(t)\right) \cdot \nabla_{\nu} \varphi\left(l_{j}-\beta q(t),-\beta \dot{q}(t)\right) \\
& =\sum_{j=1}^{\infty} \mu_{j} \nabla W\left(l_{j}-q(t)\right) \cdot \nabla_{\nu} \varphi\left(l_{j}-\beta q(t),-\beta \dot{q}(t)\right) \\
& =\ddot{q}(t) \cdot \sum_{j=1}^{\infty} \mu_{j} \nabla_{\nu} \varphi\left(l_{j}-\beta q(t),-\beta \dot{q}(t)\right) .
\end{aligned}
$$

Likewise, $T_{2}=-\ddot{q}(t) \cdot \sum_{j=1}^{\infty} \lambda_{j} \nabla_{\nu} \varphi\left(k_{j}+\alpha q(t), \alpha \dot{q}(t)\right)$.

Consequently,

$$
\begin{aligned}
& \frac{d}{d t} \int_{\mathbb{R}^{d}} \int_{\mathbb{R}^{d}} \varphi(x, v) f(t, d x, d v)=\frac{d}{d t}\left[\sum_{j=1}^{\infty} \lambda_{j} \varphi\left(k_{j}+\alpha q(t), \alpha \dot{q}(t)\right)+\mu_{j} \varphi\left(l_{j}-\beta q(t),-\beta \dot{q}(t)\right)\right] \\
& =\sum_{j=1}^{\infty}\left[\lambda_{j} \alpha \dot{q}(t) \cdot \nabla_{x} \varphi\left(k_{j}+\alpha q(t), \alpha \dot{q}(t)\right)-\mu_{j} \beta \dot{q}(t) \cdot \nabla_{x} \varphi\left(l_{j}-\beta q(t),-\beta \dot{q}(t)\right)\right] \\
& +\sum_{j=1}^{\infty}\left[\lambda_{j} \alpha \ddot{q}(t) \cdot \nabla_{v} \varphi\left(k_{j}+\alpha q(t), \alpha \dot{q}(t)\right)-\mu_{j} \beta \ddot{q}(t) \cdot \nabla_{v} \varphi\left(l_{j}-\beta q(t),-\beta \dot{q}(t)\right)\right] \\
& =\int_{\mathbb{R}^{d}} \int_{\mathbb{R}^{d}} v \cdot \nabla_{x} \varphi(x, v) f(t, d x, d v)-\beta T_{1}-\alpha T_{2}=\int_{\mathbb{R}^{d}} \int_{\mathbb{R}^{d}} v \cdot \nabla_{x} \varphi(x, v) f(t, d x, d v) \\
& -\int_{\mathbb{R}^{d}} \int_{\mathbb{R}^{d}}\left(\int_{\mathbb{R}^{d}} \nabla W(x-y) \rho(t, d y)\right) \cdot \nabla_{v} \varphi(x, v) f(t, d x, d v),
\end{aligned}
$$


so $f$ is a solution for $(\mathrm{NV})$.

Finally, note that if we replace $q(t)$ by 0 or $\xi$ in $T_{1}$ and $T_{2}$ we get $T_{1}=T_{2}=0$, so $f(-\infty)$ and $f(\infty)$ are, indeed, stationary solutions.

QED.

Corollary 6.1. The same conclusion as in Theorem 6.1 holds if one replaces $f(t, \cdot, \cdot)$ by:

$$
f(t, \cdot, \cdot):=\sum_{j=1}^{\infty}\left[\lambda_{j} \delta_{\left(k_{j}+x+\alpha q(t), \alpha \dot{q}(t)\right)}+\mu_{j} \delta_{\left(l_{j}+x-\beta q(t),-\beta \dot{q}(t)\right)}\right] .
$$

for some $x \in \mathbb{R}^{d}$ and $f(-\infty), f(\infty)$ are modified accordingly.

Proof : The right hand side of (NV) is Galilean invariant (depends only on the relative coordinates $x_{i}-x_{j}$ ).

QED.

Remark 6.4. Note that the above result settles Theorem 5.2. Indeed, given any sequence of reals $\lambda_{z} \geq 0$ such that $\sum_{z \in \mathbb{Z}^{d}} \lambda_{z}=1$, one can write $\mathbb{Z}^{d}=\left\{k_{j}\right\}_{j \geq 1}$ and count the coefficients $\lambda_{z}$ as $\bar{\lambda}_{j}$ for $z=k_{j}$. Then one takes, for example, $\lambda_{j}=\mu_{j}=\bar{\lambda}_{j} / 2$ and $l_{j}=k_{j}$ for all integers $j \geq 1$ to obtain

$$
f(-\infty)=\sum_{j=1}^{\infty} \bar{\lambda}_{j} \delta_{\left(x+k_{j}, 0\right)}=\sum_{z \in \mathbb{Z}^{d}} \lambda_{z} \delta_{(x+z, 0)} .
$$

Finally, it is not difficult to quantify the rate of approach to the equilibria; the proposition below also concludes the proof of Theorem 5.2.

Proposition 6.3. If $|\cdot|_{2 d}$ denotes the Euclidean norm in $\mathbb{R}^{2 d}$, then:

$$
W_{2}(f(t), f(-\infty)) \leq \sqrt{\alpha \beta}|(q(t), \dot{q}(t))|_{2 d}
$$

and

$$
W_{2}(f(t), f(\infty)) \leq \sqrt{\alpha \beta}|(q(t)-\xi, \dot{q}(t))|_{2 d}
$$

Proof : Let $p_{1}, \ldots, p_{n}, q_{1}, \ldots, q_{n} \in \mathscr{P}_{2}\left(\mathbb{R}^{d}\right)$ and $\theta_{j} \in[0,1], j=1, \ldots, n$ such that $\sum_{j=1}^{n} \theta_{j}=$ 1.

By taking $\gamma_{1} \in \Gamma_{o}\left(p_{1}, q_{1}\right), \gamma_{2} \in \Gamma_{o}\left(p_{2}, q_{2}\right), \ldots, \gamma_{n} \in \Gamma_{o}\left(p_{n}, q_{n}\right)$ we readily see that

$$
\sum_{j=1}^{n} \theta_{j} \gamma_{j} \in \Gamma\left(\sum_{j=1}^{n} \theta_{j} p_{j}, \sum_{j=1}^{n} \theta_{j} q_{j}\right)
$$


Thus,

$$
\begin{aligned}
W_{2}^{2}\left(\sum_{j=1}^{n} \theta_{j} p_{j}, \sum_{j=1}^{n} \theta_{j} q_{j}\right) \leq & \int_{\mathbb{R}^{d}} \int_{\mathbb{R}^{d}}|x-y|^{2}\left(\sum_{j=1}^{n} \theta_{j} \gamma_{j}\right)(d x, d y) \\
& =\sum_{j=1}^{n} \theta_{j} W_{2}^{2}\left(p_{j}, q_{j}\right) .
\end{aligned}
$$

By using the continuity of the Wasserstein distance this can easily be extended to countable convex combinations of measures, so:

$$
\begin{aligned}
W_{2}^{2}(f(t), f(-\infty)) & \leq \sum_{j=1}^{\infty}\left[\lambda_{j} W_{2}^{2}\left(\delta_{\left(k_{j}+\alpha q(t), \alpha \dot{q}(t)\right.}, \delta_{\left(k_{j}, 0\right)}\right)+\mu_{j} W_{2}^{2}\left(\delta_{\left(l_{j}-\beta q(t),-\beta \dot{q}(t)\right)}, \delta_{\left(l_{j}, 0\right)}\right)\right] \\
& =\sum_{j=1}^{\infty}\left[\lambda_{j}\left(|\alpha q(t)|^{2}+|\alpha \dot{q}(t)|^{2}\right)+\mu_{j}\left(|\beta q(t)|^{2}+|\beta \dot{q}(t)|^{2}\right)\right] \\
& =\alpha \beta\left(|q(t)|^{2}+|\dot{q}(t)|^{2}\right) .
\end{aligned}
$$

The second inequality is obtained similarly.

QED. 


\section{Chapter 7}

\section{Heteroclinic orbits for the one-dimensional, periodic Vlasov-Poisson system}

If $W$ is not differentiable everywhere, one can still give a meaning to the last term in the right hand side of the equation (5.2) in some special cases. For example, if

$$
W(x)=\frac{1}{2 d}|x|^{2}-\Phi(x),
$$

where $\Phi$ is the fundamental solution for the Laplace equation in $\mathbb{R}^{d}$, equation (5.2) can be rewritten as:

$$
\begin{aligned}
\frac{d}{d t} \int_{\mathbb{R}^{d}} \int_{\mathbb{R}^{d}} \varphi(x, v) f_{t}(d x, d v) & =\int_{\mathbb{R}^{d}} \int_{\mathbb{R}^{d}} v \cdot \nabla_{x} \varphi(x, v) f_{t}(d x, d v) \\
& -\int_{\mathbb{R}^{d}} \int_{\mathbb{R}^{d}} \nabla P_{t}(x) \cdot \nabla_{\nu} \varphi(x, v) f_{t}(d x, d v),
\end{aligned}
$$

where:

$$
-\Delta P_{t}=\rho_{t}-1
$$

This is the repulsive Vlasov-Poisson system with constant background charge (normalized to the unit) on $\mathbb{R}^{d} \times \mathbb{R}^{d}$ [27]. However, note that we are now far removed from the setting of the previous Chapters as not only is $W$ singular, but it is also non-periodic. Thus, we further specialize to $d=1$ and take the periodic potential

$$
W(z):=\frac{1}{2}\left(|z|_{\mathbb{T}^{1}}^{2}-|z|_{\mathbb{T}^{1}}\right) \text { for } z \in \mathbb{R}
$$

From Proposition 8.3 in the Appendix, if $\rho_{t}$ (the $x$-marginal of $f_{t}$ ) satisfies $\rho_{t} \ll \mathscr{L}^{1}$, then the map

$$
\Lambda(t, x):=x-\partial_{x} P(t, x)
$$


where $P(t, \cdot):=W * \rho_{t}$, is the optimal map (in the periodic setting, i.e. with respect to the cost $c(x, y)=|x-y|_{\mathbb{T}^{1}}$; see Appendix for details) such that

$$
\Lambda(t, \cdot)_{\#} \rho_{t}=\mathscr{L}_{\mathbb{T}^{1}}^{1} \text { on } \mathbb{T}^{1} \quad \text { for } t \in[0, T] .
$$

The map $P_{t}$ also satisfies (once again, see Proposition 8.3 in the Appendix)

$$
\partial_{x}^{2}\left[\frac{1}{2}|x|^{2}-P_{t}(x)\right]=\rho_{t}(x) \text { for a.e. } x \in \mathbb{R} .
$$

What if $\rho_{t}$ is not necessarily absolutely continuous with respect to the Lebesgue measure? In the section below we shall argue in favor of an appropriate modification of (VP) that will address the case of general Borel probabilities $\rho_{t} \in \mathscr{P}\left(\mathbb{T}^{1}\right)$. This adaptation has already been used in the non-periodic setting in [27].

\subsection{Barycentric projection; a periodic Vlasov-Poisson equation}

Definition 4. We say that two probabilities $f, \tilde{f}$ on $\mathbb{R}^{d} \times \mathbb{R}^{d}$ are $\mathbb{T}^{d} \times \mathbb{R}^{d}$-equivalent if :

$$
\int_{\mathbb{R}^{d}} \int_{\mathbb{R}^{d}} \varphi(x, v) f(d x, d v)=\int_{\mathbb{R}^{d}} \int_{\mathbb{R}^{d}} \varphi(x, v) \tilde{f}(d x, d v) \quad \text { for all } \varphi \in C_{b}\left(\mathbb{T}^{d} \times \mathbb{R}^{d}\right) .
$$

Remark 7.1. : Note that if $f \sim \tilde{f}$, then $\rho \sim \tilde{\rho}$, where $\rho$ and $\tilde{\rho}$ are the $x$-marginals of $f$ and $\tilde{f}$ respectively.

Definition 5. 1. If $\mu \in \mathscr{P}\left(\mathbb{R}^{d}\right), m \geq 1$ is an integer and $1 \leq p \leq \infty$ let $L_{p e r}^{p}\left(\mu ; \mathbb{R}^{m}\right):=\left\{\xi \in L^{p}\left(\mu ; \mathbb{R}^{m}\right): \xi_{n} \rightarrow \xi\right.$ in $L^{p}\left(\mu ; \mathbb{R}^{m}\right)$ for some $\left.\left\{\xi_{n}\right\}_{n} \subset C\left(\mathbb{T}^{d} ; \mathbb{R}^{m}\right)\right\}$.

We denote this set by $L_{p e r}^{p}(\mu)$ if $m=1$.

2. If $\mu \sim v$ and $\xi \in L_{p e r}^{1}\left(\mu ; \mathbb{R}^{m}\right), \zeta \in L_{p e r}^{1}\left(v ; \mathbb{R}^{m}\right)$ we say $(\mu, \xi) \sim(\nu, \zeta)$ if

$$
\int_{\mathbb{R}^{d}} \varphi \cdot \xi d \mu=\int_{\mathbb{R}^{d}} \varphi \cdot \zeta d \nu \text { for all } \varphi \in C\left(\mathbb{T}^{d} ; \mathbb{R}^{m}\right)
$$

\section{Remark 7.2.}

1. Assume $\mu \sim v$. If $\left(\xi_{n}\right)_{n} \subset C\left(\mathbb{T}^{d} ; \mathbb{R}^{m}\right)$ such that $\xi_{n} \rightarrow \xi$ in $L^{p}\left(\mu ; \mathbb{R}^{m}\right)$, then

$$
\int_{\mathbb{R}^{d}} \varphi \cdot \xi d \mu=\lim _{n \rightarrow \infty} \int_{\mathbb{R}^{d}} \varphi \cdot \xi_{n} d \mu=\lim _{n \rightarrow \infty} \int_{\mathbb{R}^{d}} \varphi \cdot \xi_{n} d \nu=\int_{\mathbb{R}^{d}} \varphi \cdot \tilde{\xi} d \nu \text { for all } \varphi \in C\left(\mathbb{T}^{d} ; \mathbb{R}^{m}\right),
$$


where $\xi_{n} \rightarrow \tilde{\xi}$ in $L^{p}\left(v ; \mathbb{R}^{m}\right)$ (indeed, since $\left\{\xi_{n}\right\}_{n}$ is Cauchy in $L^{p}\left(\mu ; \mathbb{R}^{m}\right)$, then it is easy to see that it is Cauchy in $L^{p}\left(v ; \mathbb{R}^{m}\right)$ ). Since $\mu^{*}=v^{*}$ (see Remark 6.1), we have that

$$
\int_{[0,1)^{d}} \varphi \cdot \xi d \mu^{*}=\int_{[0,1)^{d}} \varphi \cdot \tilde{\xi} d v^{*} \text { for all } \varphi \in C\left(\mathbb{T}^{d} ; \mathbb{R}^{m}\right)
$$

implies

$$
\xi \equiv \tilde{\xi}, \quad \mu^{*} \text { a.e. in } Q_{d} .
$$

2. Since $\xi \in L_{\text {per }}^{p}\left(\mu ; \mathbb{R}^{m}\right)$ is equivalent to the existence of $\left\{\xi_{n}\right\}_{n} \subset C\left(\mathbb{T}^{d} ; \mathbb{R}^{m}\right)$ such that $\xi_{n} \rightarrow \xi$ in $L^{p}\left(\mu ; \mathbb{R}^{m}\right)$, if we take any sequence $\left\{\xi_{n}\right\}_{n}$ as above we note that $\left\{\left.\xi_{n}\right|_{Q_{d}}\right\}_{n}$ is Cauchy in $L^{p}\left(\mu^{*} ; \mathbb{R}^{m}\right)$. Thus, there exists $\xi^{*} \in L^{p}\left(\mu^{*} ; \mathbb{R}^{m}\right)$ such that $\xi_{n} \rightarrow \xi^{*}$ in $L^{p}\left(\mu^{*} ; \mathbb{R}^{m}\right)$. If we take any other sequence $\left\{\zeta_{n}\right\}_{n} \subset C\left(\mathbb{T}^{d} ; \mathbb{R}^{m}\right)$ such that $\zeta_{n} \rightarrow \xi$ in $L^{p}\left(\mu ; \mathbb{R}^{m}\right)$, likewise $\left\{\left.\zeta_{n}\right|_{Q_{d}}\right\}_{n}$ is Cauchy in $L^{p}\left(\mu^{*} ; \mathbb{R}^{m}\right)$ and so $\left.\zeta_{n}\right|_{Q_{d}} \rightarrow \tilde{\xi}^{*}$ in $L^{p}\left(\mu^{*} ; \mathbb{R}^{m}\right)$. If we concatenate $\left\{\xi_{n}\right\}_{n},\left\{\zeta_{n}\right\}_{n}$ we obtain that $\tilde{\xi}^{*} \equiv \xi^{*}$, $\mu^{*}$ a.e. in $Q_{d}$, so $\xi^{*}$ depends only on $\xi$ and not on the approximating sequence. Thus, if we denote $\xi^{*}:=\xi^{*}[\mu, \xi]$, we immediately see that if $\mu \sim v$ and $\xi \in L_{\text {per }}^{p}\left(\mu ; \mathbb{R}^{m}\right), \zeta \in$ $L_{p e r}^{p}\left(v ; \mathbb{R}^{m}\right)$, then $\xi^{*}[\mu, \xi]=\xi^{*}[v, \zeta]$ if and only if $(\mu, \xi) \sim(\nu, \zeta)$.

Proposition 7.1. For any $\mu \in \mathscr{P}_{2}\left(\mathbb{R}^{d}\right)$, let $\mathrm{M}[\mu]$ be the optimal map in the periodic sense (see Appendix) such that

$$
\mathrm{M}[\mu]_{\#} \mathscr{L}_{\mathbb{T}^{d}}^{d}=\mu
$$

Then, there exists a unique map $b[\mu] \in L_{\text {per }}^{2}\left(\mu^{*} ; \mathbb{R}^{d}\right)$ such that

$$
\int_{Q_{d}}[x-\mathrm{M}[\mu](x)] \cdot \varphi(\mathrm{M}[\mu](x)) d x=\int_{Q_{d}} b[\mu](y) \cdot \varphi(y) \mu^{*}(d y),
$$

for all $\varphi \in C\left(\mathbb{T}^{d} ; \mathbb{R}^{d}\right)$.

Proof : Let $I: C\left(\mathbb{T}^{d} ; \mathbb{R}^{d}\right) \rightarrow \mathbb{R}$ given by $I \varphi=$ L.H.S. of equation (7.4). This is a linear functional on $C\left(\mathbb{T}^{d} ; \mathbb{R}^{d}\right)$ which also satisfies:

$$
|I \varphi| \leq W_{\mathbb{T}^{d}}\left(\mu, \mathscr{L}_{\mathbb{T}^{d}}^{d}\right)\|\varphi\|_{L^{2}\left(\mu ; \mathbb{R}^{d}\right)} \quad \text { for all } \varphi \in C\left(\mathbb{T}^{d} ; \mathbb{R}^{d}\right) .
$$

By Hahn-Banach, $I$ can be extended to a linear functional $\tilde{I}: L_{\text {per }}^{2}\left(\mu ; \mathbb{R}^{d}\right) \rightarrow \mathbb{R}$, continuous with

$$
|\tilde{I} \xi| \leq W_{\mathbb{T}^{d}}\left(\mu, \mathscr{L}_{\mathbb{T}^{d}}^{d}\right)\|\xi\|_{L_{p e r}^{2}\left(\mu ; \mathbb{R}^{d}\right)} .
$$

From the Riesz-Frechet representation theorem we get that there exists a unique $\xi \in$ $L_{\text {per }}^{2}\left(\mu ; \mathbb{R}^{d}\right)$ such that

$$
I \varphi=\int_{\mathbb{R}^{d}} \varphi(y) \cdot \xi(y) \mu(d y) .
$$

But we have seen that there exists a unique $\xi^{*}=\xi^{*}[\mu, \xi] \in L^{2}\left(\mu^{*} ; \mathbb{R}^{d}\right)$ such that

$$
I \varphi=\int_{Q^{d}} \varphi(y) \cdot \xi^{*}(y) \mu^{*}(d y) \text { for all } \varphi \in C\left(\mathbb{T}^{d} ; \mathbb{R}^{d}\right) .
$$

We denote $b[\mu]:=\xi^{*}$ to conclude the proof.

QED. 
Definition 6. We call $b[\mu]$ defined above the barycentric projection in the periodic sense of the optimal plan (in the periodic sense) between $\mu$ and $\left.\mathscr{L}^{d}\right|_{Q_{d}}$.

Going back to the discussion of the 1D Vlasov-Poisson system (see (VP)-(7.3)), if $\rho_{t}$ is not absolutely continuous with respect to $\mathscr{L}^{1}$, then it is a well-known fact in periodic Optimal Transport that the optimal map $\Lambda$ as in (7.2) may not exist [37], so (7.3) may not make sense. In [27] the authors argued that in the general case (i.e. $\rho_{t} \ll \mathscr{L}^{1}$ may not hold), the map $\Lambda(t, \cdot)$ should be replaced by the barycentric projection of the optimal plan between $\rho_{t}$ and $\left.\mathscr{L}^{1}\right|_{(0,1)}$ (which exists and is unique). As announced in the beginning of this Chapter, we shall argue that the same generalization is appropriate in the periodic setting. Indeed, let us prove the following:

Proposition 7.2. Assume $\mu \in \mathscr{P}_{2}\left(\mathbb{R}^{d}\right)$ such that $\mu \ll \mathscr{L}^{d}$. Let $\Phi: \mathbb{R}^{d} \rightarrow \mathbb{R}$ be the optimal map in the periodic sense such that

$$
\Phi_{\#} \mu=\mathscr{L}_{\mathbb{T}^{d}}^{d} \text { on } \mathbb{T}^{d}
$$

Then $b[\mu] \equiv \Phi-\mathrm{Id}, \mu$-a.e. in $\mathbb{R}^{d}$, where $b[\mu]$ is defined in the statement of Theorem 7.1.

Proof : It is well-known that if $\mu \ll \mathscr{L}^{d}$, and $\Phi$ is as above, then:

$$
\mathrm{M}[\mu] \circ \Phi=\mathrm{ld}, \mu \text { a.e. in } \mathbb{R}^{d}
$$

and

$$
\Phi \circ \mathrm{M}[\mu]=\mathrm{ld}, \mathscr{L}^{d} \text { a.e. in } Q_{d} .
$$

So, for all $\varphi \in C\left(\mathbb{T}^{d} ; \mathbb{R}^{d}\right)$, we have:

$$
\begin{aligned}
\int_{Q_{d}}[x-\mathrm{M}[\mu](x)] \cdot \varphi(\mathrm{M}[\mu](x)) d x & =\int_{Q_{d}}(\Phi-\mathrm{ld}) \circ \mathrm{M}[\mu](x) \cdot \varphi(\mathrm{M}[\mu](x)) d x \\
& =\int_{\mathbb{R}^{d}}[\Phi(y)-y] \cdot \varphi(y) \mu(d y) .
\end{aligned}
$$

By Proposition 7.1, we deduce $b[\mu] \equiv \Phi-\mathrm{ld}, \mu$ a.e.

QED.

We are finally in the position to make the following:

Definition 7. We say $[0, T) \ni t \mapsto \mathscr{P}(\mathbb{R} \times \mathbb{R})$ is a weak solution to the one-dimensional periodic Vlasov-Poisson equation if:

$$
[0, T] \ni t \rightarrow \int_{\mathbb{T}^{1}} \int_{\mathbb{R}} \varphi(x, v) f_{t}(d x, d v) \in W^{1,1}(0, T)
$$

and

$$
\begin{aligned}
\frac{d}{d t} \int_{\mathbb{T}^{1}} \int_{\mathbb{R}} \varphi(x, v) & =\int_{\mathbb{T}^{1}} \int_{\mathbb{R}} v \partial_{x} \varphi(x, v) f_{t}(d x, d v) \\
& +\int_{\mathbb{T}^{1}} \int_{\mathbb{R}} b\left[\rho_{t}\right](x) \partial_{x} \varphi(x, v) f_{t}(d x, d v)
\end{aligned}
$$


for a.e. $t \in[0, T]$, and $\varphi \in C_{c}\left(\mathbb{T}^{1} \times \mathbb{R}\right)$, where $\rho_{t}$ is the $x$-marginal of $f_{t}$ and $b\left[\rho_{t}\right]$ is defined in Theorem 7.1.

Remark 7.3. So, if $d=1$ and we do not know that $\rho_{t} \ll \mathscr{L}^{1}$, then $\partial_{x} P_{t}(x)=x-\Lambda_{t}(x)$ should be replaced by $-b\left[\rho_{t}\right](x)$ in the Vlassov-Poisson equation (VP). Note that we used Lemma 7.1 below to make sense of the last integral in the definition above.

Lemma 7.1. Let $\mu \in \mathscr{P}\left(\mathbb{R}^{d}\right)$ and $f \in \mathscr{P}\left(\mathbb{R}^{d} \times \mathbb{R}^{d}\right)$ such that

$$
\int_{\mathbb{R}^{d}} \int_{\mathbb{R}^{d}} \varphi(x) f(d x, d v)=\int_{\mathbb{R}^{d}} \varphi(y) \mu(d y) \text { for all } \varphi \in C\left(\mathbb{T}^{d}\right) .
$$

If $\xi \in L_{\text {per }}^{1}(\mu)$, then there exist a unique $\hat{\xi}(x, v)=\hat{\xi}(x), \hat{\xi} \in L_{\text {per }}^{1}(f)$ such that

$$
\int_{\mathbb{R}^{d}} \int_{\mathbb{R}^{d}} \hat{\xi}(x) \varphi(x) f(d x, d v)=\int_{\mathbb{R}^{d}} \xi(y) \varphi(y) \mu(d y) \quad \text { for all } \varphi \in C\left(\mathbb{T}^{d}\right) .
$$

Proof : Let $\left(\xi_{n}\right)_{n} \subset C\left(\mathbb{T}^{d}\right)$ such that $\xi_{n} \rightarrow \xi$ in $L^{1}(\mu)$. Then $\left(\xi_{n}\right)_{n}$ is Cauchy in $L^{1}(f)$ and so $\xi_{n} \rightarrow \hat{\xi}$ in $L^{1}(f)$.

As usual, $\hat{\xi}$ does not depend on the approximating sequence $\left(\xi_{n}\right)_{n}$, but only on $\xi$. Thus, we have

$$
\begin{aligned}
\int_{\mathbb{R}^{d}} \int_{\mathbb{R}^{d}} \hat{\xi}(x) \varphi(x) f(d x, d v) & =\lim _{n \rightarrow \infty} \int_{\mathbb{R}^{d}} \int_{\mathbb{R}^{d}} \xi_{n}(x) \varphi(x) f(d x, d v) \\
& =\lim _{n \rightarrow \infty} \int_{\mathbb{R}^{d}} \xi_{n}(x) \varphi(x) \mu(d x) \\
& =\int_{\mathbb{R}^{d}} \xi(x) \varphi(x) \mu(d x) .
\end{aligned}
$$

QED.

\subsection{Five kinds of trajectories. Ill-posedness for the initial-value problem. Periodic, homoclinic and heteroclinic orbits.}

In this section we prove Proposition 7.3, whose immediate consequence is Theorem 5.3. This result is of the same type as Proposition 5.1, but it is considerably more delicate to prove due to the absence of the regular potential $W$; instead one needs to (somewhat painstakingly) verify that the correct expression for the periodic barycentric projection appears in the weak form of the equation. 
Let $\sigma: \mathbb{R} \rightarrow \mathbb{R}$ given by $\sigma(t):=\frac{1}{4}(1-\cos t)$.

1. Let $m<n$ be even integers and define

$$
\sigma_{m, n}(t):= \begin{cases}0 & \text { if } t \leq m \pi \\ \sigma(t) & \text { if } m \pi<t<n \pi \\ 0 & \text { if } t \geq n \pi .\end{cases}
$$

Note that $\sigma_{m, n} \in C^{1}(\mathbb{R})$ and $\sigma_{m, n}(-\infty)=\sigma_{m, n}(\infty)=0$

2. Let $m<n$ be integers, $m$ even and $n$ odd, and define

$$
\sigma^{m, n}(t):= \begin{cases}0 & \text { if } t \leq m \pi \\ \sigma(t) & \text { if } m \pi<t<n \pi \\ \frac{1}{2} & \text { if } t \geq n \pi .\end{cases}
$$

Note that $\sigma^{m, n} \in C^{1}(\mathbb{R})$ and $\sigma^{m, n}(-\infty)=0, \sigma^{m, n}(\infty)=1 / 2$.

3. Now take two integers $m<n$ with $m$ odd and $n$ even.

$$
\bar{\sigma}^{m, n}(t):= \begin{cases}\frac{1}{2} & \text { if } t \leq m \pi \\ \sigma(t) & \text { if } m \pi<t<n \pi \\ 0 & \text { if } t \geq n \pi .\end{cases}
$$

Note that $\bar{\sigma}^{m, n} \in C^{1}(\mathbb{R})$ and $\bar{\sigma}^{m, n}(-\infty)=1 / 2, \bar{\sigma}^{m, n}(\infty)=0$.

4. If $m<n$ are both odd, let

$$
\bar{\sigma}_{m, n}(t):= \begin{cases}\frac{1}{2} & \text { if } t \leq m \pi \\ \sigma(t) & \text { if } m \pi<t<n \pi \\ \frac{1}{2} & \text { if } t \geq n \pi .\end{cases}
$$

Then $\bar{\sigma}_{m, n} \in C^{1}(\mathbb{R})$ and $\bar{\sigma}_{m, n}(-\infty)=1 / 2, \bar{\sigma}_{m, n}(\infty)=1 / 2$.

5. Let $S \subset \pi \mathbb{Z}$. If $S \neq \emptyset$, assume $S:=\left\{\ldots, n_{j} \pi, \ldots, n_{k} \pi, \ldots\right\}$ is listed in increasing order. Then, for $n_{j} \pi<t<n_{j+1} \pi$ we define $\sigma_{S}(t)=0$ if both $n_{j}$ and $n_{j+1}$ are even, or $\sigma_{S}(t)=\frac{1}{2}$ if $n_{j}$ and $n_{j+1}$ are both odd. We let $\sigma_{S}(t)=\sigma(t)$ if $n_{j}+n_{j+1}$ is odd.

Remark 7.4. Note that $\sigma_{\mathbb{Z}} \equiv \sigma$.

Proposition 7.3. Let $q: \mathbb{R} \rightarrow \mathbb{R}$ be any of the functions defined above. Then

$$
f_{t}:=\frac{1}{2}\left(\delta_{(-q,-\dot{q})}+\boldsymbol{\delta}_{(q, \dot{q})}\right)
$$

is a weak solution to the Vlasov-Poisson equation on $\mathbb{T}^{1} \times \mathbb{R}, \quad t \in \mathbb{R}$.

Proof : We begin by noting that

$$
\ddot{q}+q= \begin{cases}0 & \text { on time intervals where } q \equiv 0 \\ \frac{1}{2} & \text { on time intervals where } q \equiv \frac{1}{2} \\ \frac{1}{4} & \text { on time intervals where } q \equiv \sigma .\end{cases}
$$


Let $\varphi \in C_{c}^{1}\left(\mathbb{T}^{1} \times \mathbb{R}\right)$. Since $q \in C^{1}(\mathbb{R})$ and $\dot{q}$ is absolutely continuous, we see that

$$
\int_{\mathbb{T}^{1}} \int_{\mathbb{R}} \varphi(x, v) f_{t}(d x, d v)=\frac{1}{2}[\varphi(-q(t),-\dot{q}(t))+\varphi(q(t), \dot{q}(t))]
$$

is absolutely continuous as a function of time. We have:

$$
\begin{aligned}
\frac{d}{d t} \int_{\mathbb{T}^{1}} \int_{\mathbb{R}} \varphi(x, v) f_{t}(d x, d v) & =\frac{1}{2}\left\{\left[-\dot{q} \partial_{x} \varphi(-q,-\dot{q})+\dot{q} \partial_{x} \varphi(q(t), \dot{q}(t))\right]\right. \\
& \left.+\left[-\ddot{q} \partial_{\nu} \varphi(-q,-\dot{q})+\ddot{q} \partial_{\nu} \varphi(q(t), \dot{q}(t))\right]\right\} .
\end{aligned}
$$

Clearly,

$$
\int_{\mathbb{T}^{1}} \int_{\mathbb{R}} v \partial_{x} \varphi(x, v) f_{t}(d x, d v)=\frac{1}{2}\left[-\dot{q} \partial_{x} \varphi(-q,-\dot{q})+\dot{q} \partial_{x} \varphi(q(t), \dot{q}(t))\right] .
$$

As usual, denote by

$$
\rho_{t}:=\frac{1}{2}\left(\delta_{-q(t)}+\delta_{q(t)}\right)
$$

the $x$-marginal of $f_{t}$.

(1) If $q(t)=0$ for some $t \in \mathbb{R}$, then $\rho_{t}=\delta_{0}$. The optimal map pushing $\mathscr{L}_{\mathbb{T}^{1}}^{1}$ forward to $\delta_{0}$ in the periodic sense is given by

$$
M_{0}(x)= \begin{cases}0 & \text { if } 0 \leq x<\frac{1}{2} \\ 1 & \text { if } x \leq \frac{1}{2}<1\end{cases}
$$

Indeed, note that $M_{0}$ satisfies $M_{0 \#} \mathscr{L}_{\mathbb{T}^{1}}^{1}=\delta_{0}$, the extension to $\mathbb{R}$ by $M_{0}(x+k)=$ $M_{0}(x)+k(x \in[0,1), k \in \mathbb{Z})$ is nondecreasing, and $\left|M_{0}(x)-x\right| \leq \frac{1}{2}$ for all $x \in[0,1)$ (see Remark 8.1). So, we have:

$$
\begin{aligned}
\int_{0}^{1}\left[x-M_{0}(x)\right] \zeta\left(M_{0}(x)\right) d x & =\int_{0}^{\frac{1}{2}} x \zeta(0) d x+\int_{\frac{1}{2}}^{1}(x-1) \zeta(1) d x \\
& =0 \quad \text { if } \zeta \in C\left(\mathbb{T}^{1}\right) .
\end{aligned}
$$

So,

$$
\int_{\mathbb{R}} b\left[\delta_{0}\right](y) \varphi(y) \delta_{0}(y)=b\left[\delta_{0}\right](0) \varphi(0)=0 \quad \text { for all } \varphi \in C\left(\mathbb{T}^{1}\right),
$$

i.e. $b\left[\delta_{0}\right](0)=0$, or $b\left[\rho_{t}\right] \equiv 0, \rho_{t}$-a.e.. Therefore, on all subintervals of $\mathbb{R}$ where $q \equiv 0$, we have

$$
\ddot{q}(t)=0=b\left[\delta_{0}\right](q(t))=b\left[\delta_{0}\right](0) .
$$

(2) Next, if $t$ belongs to a subinterval on which $q(t)=\frac{1}{2}$, we have $\rho_{t}=\frac{1}{2}\left(\delta_{-\frac{1}{2}}+\right.$ $\left.\delta_{\frac{1}{2}}\right) \sim_{\mathbb{T}^{1}} \delta_{\frac{1}{2}}$.

The optimal map in the periodic sense pushing $\mathscr{L}_{\mathbb{T}^{1}}^{1}$ forward to $\delta_{\frac{1}{2}}$ is $M \equiv \frac{1}{2}$ on $[0,1)$. 
Indeed, the extension to $\mathbb{R}$ by $M(x+k)=M(x)+k(x \in[0,1), k \in \mathbb{Z})$ is nondecreasing, satisfies $M_{\#} \mathscr{L}_{\mathbb{T}^{1}}^{1}=\delta_{\frac{1}{2}}$, and

$$
|x-M(x)|=\left|x-\frac{1}{2}\right| \leq \frac{1}{2} \text { for all } x \in[0,1)
$$

Thus,

$$
\int_{0}^{1}[x-M(x)] \zeta(M(x)) d x=\int_{0}^{1}\left(x-\frac{1}{2}\right) \zeta\left(\frac{1}{2}\right) d x=0
$$

so, as before, we deduce $b\left[\rho_{t}\right] \equiv 0, \rho_{t}$ a.e.

(3) Finally, if $t$ belongs to an open subinterval of $\mathbb{R}$ on which $q \equiv \sigma$, then we see that $0<q(t)<\frac{1}{2}$. Denote $\alpha:=q(t)$. We have $\rho_{t}=\frac{1}{2}\left(\delta_{-\alpha}+\delta_{\alpha}\right)$.

Let

$$
M(x)= \begin{cases}\alpha & \text { if } 0 \leq x<\frac{1}{2} \\ 1-\alpha & \text { if } \frac{1}{2} \leq x<1 .\end{cases}
$$

Since $0<\alpha<\frac{1}{2}, M$ is nondecreasing. Let, $\zeta \in C\left(\mathbb{T}^{1}\right)$ and compute

$$
\begin{aligned}
\int_{0}^{1} \zeta(M(x)) d x & =\int_{0}^{\frac{1}{2}} \zeta(\alpha) d x+\int_{\frac{1}{2}}^{1} \zeta(1-\alpha) d x \\
& =\frac{1}{2}[\zeta(-\alpha)+\zeta(\alpha)]=\int_{\mathbb{R}} \zeta(y) \rho_{t}(d y),
\end{aligned}
$$

so $M_{\#} \mathscr{L}_{\mathbb{T}^{1}}^{1}=\rho_{t}$. Furthermore,

$$
|x-M(x)|= \begin{cases}|x-\alpha| \leq \frac{1}{2} & \text { if } 0 \leq x<\frac{1}{2} \\ |x+\alpha-1| \leq \frac{1}{2} & \text { if } \frac{1}{2} \leq x<1\end{cases}
$$

and the extension to $\mathbb{R}$ by $M(x+k)=M(x)+k(x \in[0,1), k \in \mathbb{Z})$ is nondecreasing, so $M$ is the optimal map in the periodic sense such that $M_{\#} \mathscr{L}_{\mathbb{T}^{1}}^{1}=\rho_{t}$. We now look at (for $\zeta \in C\left(\mathbb{T}^{1}\right)$ )

$$
\begin{aligned}
\int_{0}^{1}[x-M(x)] \zeta(M(x)) d x & =\int_{0}^{\frac{1}{2}}(x-\alpha) \zeta(\alpha) d x+\int_{\frac{1}{2}}^{1}(x+\alpha-1) \zeta(-\alpha) d x \\
& =\frac{1}{2}[\zeta(-\alpha)+\zeta(\alpha)]=\left(\frac{1}{8}-\frac{\alpha}{2}\right) \zeta(\alpha)+\left(-\frac{1}{8}+\frac{\alpha}{2}\right) \zeta(-\alpha) \\
& =\frac{1}{2}\left[\left(\alpha-\frac{1}{4}\right) \zeta(-\alpha)+\left(-\alpha+\frac{1}{4}\right) \zeta(\alpha)\right]
\end{aligned}
$$

to see that

$$
b\left[\rho_{t}\right](-\alpha)=\alpha-\frac{1}{4}, b\left[\rho_{t}\right](\alpha)=-\alpha+\frac{1}{4}
$$


Since $\alpha=q(t)$ and $\ddot{q}(t)+q(t)=\frac{1}{4}$ for all $t$ in open subintervals where $q(t) \equiv \sigma(t)$, we see that

$$
b\left[\rho_{t}\right](-q(t))=-\ddot{q}(t) \text { and } b\left[\rho_{t}\right](q(t))=\ddot{q}(t) .
$$

Thus,

$$
\frac{1}{2}\left[-\ddot{q} \partial_{\nu} \varphi(-q,-\dot{q})+\ddot{q} \partial_{v} \varphi(q, \dot{q})\right]=\int_{\mathbb{T}^{1}} \int_{\mathbb{R}} b\left[\rho_{t}\right](x) \partial_{v} \varphi(x, v) f_{t}(d x, d v),
$$

which finishes the proof.

QED.

Remark 7.5. Note that the statements collected in Theorem 5.3 are immediate consequences of Proposition 7.3. 


\section{Chapter 8}

\section{Appendix}

\subsection{A new inner-product and its corresponding gradi- ent}

Proposition 8.1. On $\mathbb{R}^{d \times(n-1)}$ we define the inner-product

$$
\left\langle\left\langle Y^{1}, Y^{2}\right\rangle\right\rangle:=\frac{1}{n^{2}} \sum_{j=1}^{n-1} y_{j}^{1} \cdot y_{j}^{2}+\frac{1}{2 n^{2}} \sum_{j, k=1}^{n-1}\left(y_{j}^{1}-y_{k}^{1}\right) \cdot\left(y_{j}^{2}-y_{k}^{2}\right) \text { for all } Y^{1}, Y^{2} \in \mathbb{R}^{d \times(n-1)} .
$$

Then the expression in the right hand side of (5.11) is the gradient of

$$
\tilde{\mathscr{W}}^{n}: \mathbb{R}^{d \times(n-1)} \rightarrow \mathbb{R}, \tilde{\mathscr{W}}^{n}(Y):=\frac{1}{n^{2}} \sum_{j=1}^{n-1} W\left(y_{j}\right)+\frac{1}{2 n^{2}} \sum_{j, k=1}^{n-1} W\left(y_{j}-y_{k}\right)
$$

with respect to $\langle\langle\cdot, \cdot\rangle\rangle$.

Proof: We need to show $\left\langle\nabla \tilde{\mathscr{W}}^{n}(Y), \xi\right\rangle=\langle\langle U, \xi\rangle\rangle$ for all $\xi \in \mathbb{R}^{d \times(n-1)}$, where $U$ is the matrix whose columns are the vectors in the right hand side of $(5.11)$, and $\langle\cdot, \cdot\rangle$ denotes the standard, Euclidean inner-product. First,

$$
\begin{aligned}
\left\langle\nabla \tilde{\mathscr{W}}^{n}(Y), \xi\right\rangle & =\sum_{j=1}^{n-1} \nabla y_{j} \tilde{\mathscr{W}}^{n}(Y) \cdot \xi_{j} \\
& =\frac{1}{n^{2}} \sum_{j=1}^{n-1}\left[\nabla W\left(y_{j}\right)+\sum_{k=1}^{n-1} \nabla W\left(y_{j}-y_{k}\right)\right] \cdot \xi_{j}
\end{aligned}
$$


Next we compute $\langle\langle U, \xi\rangle\rangle$ :

$$
\begin{aligned}
\langle\langle U, \xi\rangle\rangle & =\frac{1}{n^{2}} \sum_{j=1}^{n-1} u_{j} \cdot \xi_{j}+\frac{1}{2 n^{2}} \sum_{j, k=1}^{n-1}\left(u_{j}-u_{k}\right) \cdot\left(\xi_{j}-\xi_{k}\right) \\
& =\frac{1}{n^{2}} \sum_{j=1}^{n-1} u_{j} \cdot \xi_{j}+\frac{1}{2 n^{2}} \sum_{j, k=1}^{n-1}\left(u_{j} \cdot \xi_{j}+u_{k} \cdot \xi_{k}\right)-\frac{1}{2 n^{2}} \sum_{j, k=1}^{n-1}\left(u_{j} \cdot \xi_{k}+u_{k} \cdot \xi_{j}\right) \\
& =\frac{1}{n^{2}} \sum_{j=1}^{n-1} u_{j} \cdot \xi_{j}+\frac{n-1}{n^{2}} \sum_{j=1}^{n-1} u_{j} \cdot \xi_{j}-\frac{1}{2 n^{2}} \sum_{j, k=1}^{n-1}\left(u_{j} \cdot \xi_{k}+u_{k} \cdot \xi_{j}\right) \\
& =\frac{1}{n} \sum_{j=1}^{n-1} u_{j} \cdot \xi_{j}-\frac{1}{n^{2}}\left(\sum_{j=1}^{n-1} u_{j}\right) \cdot\left(\sum_{j=1}^{n-1} \xi_{j}\right) \\
& =\frac{1}{n} \sum_{j=1}^{n-1} u_{j} \cdot \xi_{j}-\sum_{j=1}^{n-1} \xi_{j} \cdot\left(\frac{1}{n^{2}} \sum_{j=1}^{n-1} u_{j}\right) \\
& =\frac{1}{n^{2}} \sum_{j=1}^{n-1} \xi_{j} \cdot\left(n u_{j}-\sum_{k=1}^{n-1} u_{k}\right), \quad \text { for all } U, \xi \in \mathbb{R}^{d \times(n-1)} .
\end{aligned}
$$

Now, fix $Y \in \mathbb{R}^{d \times(n-1)}$ and use the expression in the right hand side of (5.11) to set:

$$
u_{j}=\frac{1}{n} \nabla W\left(y_{j}\right)+\frac{1}{n} \sum_{k=1}^{n-1}\left[\nabla W\left(y_{k}\right)+\nabla W\left(y_{j}-y_{k}\right)\right] .
$$

Thus,

$$
\begin{aligned}
\sum_{j=1}^{n-1} u_{j} & =\sum_{j=1}^{n-1}\left[\frac{1}{n} \nabla W\left(y_{j}\right)+\frac{1}{n} \sum_{k=1}^{n-1}\left[\nabla W\left(y_{k}\right)+\nabla W\left(y_{j}-y_{k}\right)\right]\right] \\
& =\sum_{j=1}^{n-1} \nabla W\left(y_{j}\right)+\frac{1}{n} \sum_{j, k=1}^{n-1} \nabla W\left(y_{j}-y_{k}\right)=\sum_{j=1}^{n-1} \nabla W\left(y_{j}\right) .
\end{aligned}
$$

Since

$$
n u_{j}=\nabla W\left(y_{j}\right)+\sum_{k=1}^{n-1} \nabla W\left(y_{k}\right)+\sum_{k=1}^{n-1} \nabla W\left(y_{j}-y_{k}\right)
$$

we get

$$
n u_{j}-\sum_{j=1}^{n-1} u_{j}=\nabla W\left(y_{j}\right)+\sum_{k=1}^{n-1} \nabla W\left(y_{j}-y_{k}\right)
$$

We conclude

$$
\left\langle\nabla \tilde{\mathscr{W}}^{n}(Y), \xi\right\rangle=\frac{1}{n^{2}} \sum_{j=1}^{n-1} \xi_{j} \cdot\left[\nabla W\left(y_{j}\right)+\sum_{k=1}^{n-1} \nabla W\left(y_{j}-y_{k}\right)\right]=\langle\langle U, \xi\rangle\rangle .
$$

QED. 


\subsection{Optimal Transport on the torus}

There are different (yet, equivalent) ways of defining $\mathscr{P}\left(\mathbb{T}^{d}\right)$, the space of Borel probability measures on $\mathbb{T}^{d}$.

1. In [23], which is the original paper on periodic Optimal Transport, the following procedure is used: let $\mu^{*} \in \mathscr{P}\left(Q_{d}\right)$. Define $\mu_{0} \in \mathscr{P}\left(\mathbb{R}^{d}\right)$ by $\mu_{0}(B):=\mu^{*}\left(B \cap Q_{d}\right)$ for any Borel set $B$. Then define the translates of $\mu_{0}$ by

$$
\mu_{k}(B):=\mu_{0}(B-k) \text { for all } k \in \mathbb{Z}^{d} .
$$

Clearly, $\mu_{k}\left(Q_{d}+k\right)=1$. Let

$$
\mu:=\sum_{k \in \mathbb{Z}^{d}} \mu_{k}
$$

This is a Borel measure on $\mathbb{R}^{d}$ with $\mu\left(\mathbb{R}^{d}\right)=\infty$, and such that for any $\varphi \in C\left(\mathbb{T}^{d}\right)$ we have

$$
\int_{Q[a]} \varphi d \mu \text { is independent of } a:=\left(a_{1}, \ldots, a_{d}\right) \in \mathbb{R}^{d}, \text { where } Q[a]:=\prod_{j=1}^{d}\left[a_{j}, a_{j}+1\right) .
$$

We denote the value of this integral by

$$
\int_{\mathbb{T}^{d}} \varphi d \mu\left(=\int_{Q_{d}} \varphi d \mu^{*}\right)
$$

Take $\varphi \equiv 1$ to see that

$$
\mu\left(\mathbb{T}^{d}\right):=\int_{\mathbb{T}^{d}} 1 d \mu=1 .
$$

It is known [23] that if $\mu, v \in \mathscr{P}\left(\mathbb{T}^{d}\right)$ (the set of all measures defined as above) and $\mu \ll \mathscr{L}^{d}$, then there exists a unique $\Phi: \mathbb{R}^{d} \rightarrow \mathbb{R}$ convex such that $\Phi-\frac{1}{2}|\mathrm{Id}|^{2}$ is periodic and $\nabla \Phi_{\#} \mu=\nu$ in the periodic sense, i.e.

$$
\int_{\mathbb{T}^{d}} \xi(\nabla \Phi(x)) \mu(d x)=\int_{\mathbb{T}^{d}} \xi(y) v(d y) \quad \text { for all } \xi \in C\left(\mathbb{T}^{d}\right) .
$$

Furthermore,

$$
|\nabla \Phi(x)-x| \leq \frac{\sqrt{d}}{2} \quad \text { for a.e. } x \in \mathbb{R}^{d}
$$

and

$$
W_{\mathbb{T}^{d}}^{2}(\mu, v):=\int_{\mathbb{T}^{d}}|\nabla \Phi(x)-x|^{2} \mu(d x)=\min _{S_{\#} \mu=v} \int_{\mathbb{T}^{d}}|S(x)-x|^{2} \mu(d x),
$$

where $S$ satisfies $S(x+k)=S(x)+k$ for a.e. $x \in \mathbb{R}^{d}$, for all $k \in \mathbb{Z}^{d}$ and

$$
\int_{\mathbb{T}^{d}} \xi(S(x)) \mu(d x)=\int_{\mathbb{T}^{d}} \xi(y) v(d y) \quad \text { for all } \xi \in C\left(\mathbb{T}^{d}\right) .
$$


2. We now describe the procedure from [26], which we briefly touched upon in the beginning of the previous Chapter. If $\mu \in \mathscr{P}\left(\mathbb{R}^{d}\right)$, then $\mathscr{P}\left(\mathbb{T}^{d}\right):=\mathscr{P}\left(\mathbb{R}^{d}\right) / \sim$, where

$$
\mu \sim \nu \Longleftrightarrow \int \varphi d \mu=\int \varphi d v \text { for all } C\left(\mathbb{T}^{d}\right)
$$

If $\varphi \in C\left(\mathbb{T}^{d}\right)$ and $\mu \in \mathscr{P}\left(\mathbb{R}^{d}\right)$, then:

$$
\int_{\mathbb{R}^{d}} \varphi d \mu=\sum_{k \in \mathbb{Z}^{d}} \int_{Q_{d}+k} \varphi d \mu=\sum_{k \in \mathbb{Z}^{d}} \int_{Q_{d}} \varphi d \mu_{k}
$$

Here,

$$
\mu_{k}(B):=\mu(B+k) \quad \text { for all } k \in \mathbb{Z}^{d} \text { and } B \subset Q_{d}
$$

Let

$$
\mu^{*}:=\sum_{k \in \mathbb{Z}^{d}} \mu_{k} \in \mathscr{P}\left(Q_{d}\right)
$$

to see that $\mu^{*} \sim \mu$. The distance between $\mu, v$ is (see [26] for a proof of the second equality)

$$
\mathscr{W}_{\mathbb{T}^{d}}(\mu, v)=\inf _{\substack{\mu \sim \tilde{\mu} \\ v \sim \tilde{v}}} W_{2}(\tilde{\mu}, \tilde{v})=\min _{v \sim \tilde{v}} W_{2}\left(\mu^{*}, \tilde{v}\right)
$$

where $W_{2}$ is the Wasserstein distance for quadratic cost $c(x, y):=|x-y|^{2} / 2$ and $\mathscr{W}_{\mathbb{T}^{d}}$ is the distance associated with the cost $c_{\mathbb{T}^{d}}(x, y):=|x-y|_{\mathbb{T}^{d}}^{2}$ (see [26]). If $\mu \ll \mathscr{L}^{d}$, then $\left.\mu^{*} \ll \mathscr{L}^{d}\right|_{Q_{d}}$. Since spt $\mu^{*} \subset \bar{Q}_{d}$, it follows [23] that for each $\tilde{v} \sim v$, there exists $S[\tilde{v}]: Q_{d} \rightarrow \mathbb{R}$ the gradient of a convex function $\Phi$ such that $S[\tilde{v}]_{\#} \mu^{*}=\tilde{v}$, i.e.

$$
\int_{Q_{d}} \xi(S[\tilde{v}](x)) \mu^{*}(d x)=\int_{\mathbb{R}^{d}} \xi(y) \tilde{v}(d y) \quad \text { for all } \xi \in C_{b}\left(\mathbb{R}^{d}\right) \text {. }
$$

Also,

$$
\int_{Q_{d}}|S[\tilde{v}](x)-x|^{2} \mu^{*}(d x)=W_{2}^{2}\left(\mu^{*}, \tilde{v}\right)
$$

Let $v_{0} \sim v$ (whose existence follows from (8.2)) such that

$$
\int_{Q_{d}}\left|S\left[v_{0}\right](x)-x\right|^{2} \mu^{*}(d x)=W_{\mathbb{T}^{d}}^{2}\left(\mu^{*}, v_{0}\right)=W_{2}^{2}\left(\mu^{*}, v_{0}\right)
$$

The equivalence with the previous way (from [23], explained at $\mathbf{1}$ above) of viewing Optimal Transport on the torus is obtained from:

Proposition 8.2. There exists a convex function $\Phi: \mathbb{R}^{d} \rightarrow \mathbb{R}$ such that $\Phi-\frac{1}{2}|\mathrm{Id}|^{2}$ is $\mathbb{Z}^{d}$-periodic and $\left.\nabla \Phi\right|_{Q_{d}}=S\left[v_{0}\right], \mu^{*}$-a.e. in $Q_{d}$. Also, $\mathscr{W}_{\mathbb{T}^{d}}(\mu, v)=W_{\mathbb{T}^{d}}(\mu, v)$. 
Proof: According to [26] (section 2.2), we have

$$
\begin{aligned}
\inf _{\gamma \in \Gamma\left(\mu^{*}, v_{0}\right)} \int_{\mathbb{R}^{2 d}}|x-y|_{\mathbb{T}^{d}}^{2} \gamma(d x, d y) & =: \mathscr{W}_{\mathbb{T}^{d}}^{2}(\mu, v)=\mathscr{W}_{\mathbb{T}^{d}}^{2}\left(\mu^{*}, v_{0}\right)=W_{2}^{2}\left(\mu^{*}, v_{0}\right) \\
& =\max _{(f, g) \in \mathscr{U}}\left\{\int_{\mathbb{R}^{d}} f d \mu^{*}+\int_{\mathbb{R}^{d}} g d v_{0}\right\},
\end{aligned}
$$

where $\mathscr{U}$ is the set of all $(f, g) \in C\left(\mathbb{T}^{d}\right) \times C\left(\mathbb{T}^{d}\right)$ such that

$$
f(x)=\sup _{u \in \mathbb{R}^{d}}\left\{|x-u|_{\mathbb{T}^{d}}^{2}-g(u)\right\}, g(y)=\sup _{v \in \mathbb{R}^{d}}\left\{|y-v|_{\mathbb{T}^{d}}^{2}-f(v)\right\} \text { for all } x, y \in \mathbb{R}^{d} .
$$

From the definition of $W_{2}$ (see Definition (2)), the inequality $c \geq c_{\mathbb{T}^{d}}$ and the fact that the optimal transport plan (with respect to $c$ ) is uniquely given by $\bar{\gamma}:=(\mathrm{Id} \times$ $\left.S\left[v_{0}\right]\right)_{\#} \mu^{*}$, we deduce

$$
\bar{f}(x)+\bar{g}\left(S\left[v_{0}\right](x)\right)=\left|x-S\left[v_{0}\right](x)\right|^{2}=\left|x-S\left[v_{0}\right](x)\right|_{\mathbb{T}^{d}}^{2} \text { for } \mu^{*} \text { a.e. } x \in Q_{d},
$$

where $(\bar{f}, \bar{g})$ is a maximizing pair in $\mathscr{U}$. It follows that there exists a convex function $\Phi: \mathbb{R}^{d} \rightarrow \mathbb{R}$ such that $\Phi-\frac{1}{2}|\mathrm{Id}|^{2}=\bar{f}$ is $\mathbb{Z}^{d}$-periodic and $\left(\Phi^{*}\right.$ is the Legendre transform of $\Phi)$

$$
x \cdot S\left[v_{0}\right](x)=\Phi(x)+\Phi^{*}\left(S\left[v_{0}\right](x)\right) \text { for } \mu^{*} \text { a.e. } x \in Q_{d} .
$$

This means $S\left[v_{0}\right] \equiv \nabla \Phi, \mu^{*}$-a.e. in $Q_{d}$. By the uniqueness of $\Phi$ from $\mathbf{1}$, we get that $\Phi$ obtained here is the same as that from 1. In particular, $W_{\mathbb{T}^{d}}=\mathscr{W}_{\mathbb{T}^{d}}$.

QED.

Remark 8.1. It follows from the considerations above that in order to determine if some map $S: Q_{d} \rightarrow \mathbb{R}^{d}$ that pushes $\mu^{*}$ forward to $v$ in the periodic sense is also optimal in the periodic sense, we need to check both (1) that $|S(x)-x| \leq \sqrt{d} / 2$ for $\mu$-a.e. $x \in Q_{d}$, and (2) the fact that the extension $\tilde{S}$ given by $\tilde{S}(x+k):=S(x)+k$, $x \in Q_{d}, k \in \mathbb{Z}^{d}$ is the gradient of a convex function defined on $\mathbb{R}^{d}$.

Lemma 8.1. Let $\rho \in \mathscr{P}\left(\mathbb{T}^{1}\right) \cap C\left(\mathbb{T}^{1}\right)$ and let $W$ be the kernel defined in (7.1). Then $P^{*}(x):=W * \rho^{*}(x)$ satisfies

$$
\frac{d P^{*}}{d x}(x)=x-\int_{0}^{x} \rho^{*}(z) d z+\frac{1}{2}-\int_{0}^{1} z \rho^{*}(z) d z
$$

and

$$
\frac{d^{2}}{d x^{2}}\left[\frac{1}{2}|x|^{2}-P^{*}(x)\right]=\rho^{*}(x) .
$$

for all $x \in[0,1)$.

We shall skip the proof of the above lemma, as it follows easily from the computation of the distributional derivatives of the kernel $W$. 
Proposition 8.3. If $\rho^{*} \in \mathscr{P}([0,1))$ and $\rho^{*} \ll \mathscr{L}^{1}$, then $P^{*}$ is differentiable $\rho^{*}$-a.e. in $[0,1)$ and the map $G: \mathbb{R} \rightarrow \mathbb{R}$ given by $G(x):=x-\left(P^{*}\right)^{\prime}(x)$ is the optimal map such that:

$$
G_{\#} \rho=\mathscr{L}_{\mathbb{T}^{1}}^{1} \text { on } \mathbb{T}^{1},
$$

i.e. $G: \mathbb{T}^{1} \rightarrow \mathbb{T}^{1}$ is the optimal map (with respect to quadratic cost) such that

$$
\int_{\mathbb{T}^{1}} \zeta(G(x)) \rho(d x)=\int_{\mathbb{T}^{1}} \zeta(y) d y
$$

for all $\zeta \in C\left(\mathbb{T}^{1}\right)$ and for all $\rho \sim \rho^{*}$.

Proof: Since $\rho^{*} \in L^{1}(0,1)$, there exists $\left(\tilde{\rho}_{n}\right)_{n} \subset C_{c}(0,1)$ such that $\tilde{\rho}_{n} \rightarrow \rho^{*}$ in $L^{1}(0,1)$. From the triangle inequality ||$a|-| b|| \leq|a-b|$ we deduce $\left|\tilde{\rho}_{n}\right| \rightarrow \rho^{*}$ in $L^{1}(0,1)$. Take

$$
\rho_{n}(x):=\frac{\left|\tilde{\rho}_{n}(x)\right|+\frac{1}{n}}{\frac{1}{n}+\int_{0}^{1}\left|\tilde{\rho}_{n}(y)\right| d y} \text { for all integers } n \geq 1, \text { and all } x \in[0,1] .
$$

Note that $\rho_{n}(0)=\rho_{n}(1)$ and $\rho_{n}$ integrates to 1 over $[0,1]$, so $\rho_{n} \in \mathscr{P}([0,1)) \cap C\left(\mathbb{T}^{1}\right)$. Let

$$
F_{n}(x):=\int_{0}^{x} \rho_{n}(y) d y, F(x):=\int_{0}^{x} \rho(y) d y \text { for all } x \in[0,1] .
$$

It is not difficult to see that $\rho_{n}$ converges to $\rho^{*}$ in $L^{1}(0,1)$, which implies that $F_{n} \rightarrow$ $F$ uniformly in $[0,1]$. Since $\rho_{n}>0$ on $[0,1]$ and $\rho_{n} \in C(0,1)$ we have that $F_{n}$ : $[0,1] \rightarrow[0,1]$ with $F_{n} \in C^{1}([0,1]), F_{n}$ strictly increasing and $F_{n}(0)=0, F_{n}(1)=1$ for all integers $n \geq 1$. If we let $P_{n}(x):=W * \rho_{n}(x), x \in[0,1]$, we see that

$$
P_{n}(x)=\int_{0}^{1}\left[(z-x)^{2}-|z-x|\right] \rho_{n}(z) d z
$$

for all $x \in[0,1]$ because $|z-x| \leq 1$ for all $z, x \in[0,1]$ and we can see (simple computation) that, in this case,

$$
|z-x|_{\mathbb{T}^{1}}^{2}-|z-x|_{\mathbb{T}^{1}}=|z-x|^{2}-|z-x| .
$$

We then use (8.3) to see that

$$
\begin{aligned}
P_{n}^{\prime}(x) & =x+\frac{1}{2}-F_{n}(x)-\int_{0}^{1} z F_{n}^{\prime}(z) d z \\
& =x-\frac{1}{2}-F_{n}(x)+\int_{0}^{1} F_{n}(z) d z .
\end{aligned}
$$

To prove that $\left|P_{n}^{\prime}(x)\right| \leq \frac{1}{2}$ for all $x \in(0,1)$ we show that

$$
0 \leq x+\frac{1}{2}-F_{n}(x)+\int_{0}^{1} F_{n}(z) d z \leq 1 .
$$


We will prove that

$$
0 \leq x-f(x)+\int_{0}^{1} f(z) d z \leq 1
$$

for any strictly increasing, continuous function

$$
f:[0,1] \rightarrow[0,1] \text { such that } f(0)=0, f(1)=1 .
$$

The obvious identity

$$
\int_{0}^{1} f(x) d x+\int_{0}^{1} f^{-1}(y) d y=1
$$

shows that it is enough to prove

$$
f(x)-x \leq \int_{0}^{1} f(z) d z \quad \text { for all } x \in[0,1],
$$

as the second inequality in (8.6) follows by applying the inequality above to $f^{-1}$. Since $f$ is increasing, we have that for any $x \in[0,1]$,

$$
\int_{0}^{1} f(z) d z \geq \int_{x}^{1} f(z) d z \geq \int_{x}^{1} f(x) d z=(1-x) f(x) .
$$

But $0 \leq f(x) \leq 1$ and $0 \leq x \leq 1$, so $(1-x) f(x) \geq f(x)-x$, which proves our inequality.

So,

$$
\left|x-\left[F_{n}(x)+\frac{1}{2}-\int_{0}^{1} F_{n}(z) d z\right]\right|=\left|P_{n}^{\prime}(x)\right| \leq \frac{1}{2} .
$$

Denote

$$
G_{n}(x):=F_{n}(x)+\frac{1}{2}-\int_{0}^{1} F_{n}(z) d z
$$

which is also strictly increasing on $[0,1]$, so it is the gradient of a convex function. Compute:

$$
\int_{0}^{1} \zeta\left(G_{n}(x)\right) \rho_{n}(x) d x=\int_{a_{n}}^{b_{n}} \zeta(y) \rho_{n}\left(G_{n}^{-1}(y)\right)\left(G_{n}^{-1}\right)^{\prime}(y) d y
$$

where $a_{n}:=\frac{1}{2}-\int_{0}^{1} F_{n}(z) d z, b_{n}:=\frac{3}{2}-\int_{0}^{1} F_{n}(z) d z$. Since,

$$
\left(G_{n}^{-1}\right)^{\prime}(y)=\frac{1}{G_{n}^{\prime}\left(G_{n}^{-1}(y)\right)}=\frac{1}{\rho_{n}\left(G_{n}^{-1}(y)\right)} \quad \text { for all } a_{n} \leq y \leq b_{n},
$$

we obtain,

$$
\int_{0}^{1} \zeta\left(G_{n}(x)\right) \rho_{n}(x) d x=\int_{a_{n}}^{b_{n}} \zeta(y) d y=\int_{0}^{1} \zeta(y) d y \text { if } \zeta \in C\left(\mathbb{T}^{1}\right) .
$$


If we extend $G_{n}$ to $\mathbb{R}$ by

$$
\tilde{G}_{n}(x):=G_{n}(x-k)+k \text { if } k \leq x<k+1(k \in \mathbb{Z}),
$$

then

$$
\int_{\mathbb{T}^{1}} \zeta\left(\tilde{G}_{n}(x)\right) \hat{\rho}_{n}(x) d x=\int_{\mathbb{T}^{1}} \zeta(y) d y \quad \text { for all } \zeta \in C\left(\mathbb{T}^{1}\right)
$$

where $\hat{\rho}_{n}$ is periodic extention of $\rho_{n}$. Since $G_{n}(1)=G_{n}(0)+1, \tilde{G}_{n}$ is strictly increasing, so (see Remark 8.1) $\tilde{G}_{n}$ is the gradient of a convex function such that $\tilde{G}_{n}: \mathbb{T}^{1} \rightarrow \mathbb{T}^{1}$ and $\left|x-\tilde{G}_{n}(x)\right| \leq \frac{1}{2}$ for all $x \in \mathbb{R}$, and $\tilde{G}_{n \#} \hat{\rho}_{n}=\mathscr{L}_{\mathbb{T}^{1}}^{1}$. We deduce that $\tilde{G}_{n}$ is the optimal map such that $\tilde{G}_{n \# \hat{\rho}_{n}}=\mathscr{L}_{\mathbb{T}^{1}}^{1}$ on $\mathbb{T}^{1}$. We now let $n \rightarrow \infty$ and use $\rho_{n} \rightarrow \rho^{*}$ in $L^{1}(0,1)$ and $F_{n} \rightarrow F$ uniformly on $[0,1]$ to deduce

$$
\int_{0}^{1} \zeta(G(x)) \rho^{*}(x) d x=\int_{0}^{1} \zeta(y) d y \quad \text { for all } \zeta \in C\left(\mathbb{T}^{1}\right),
$$

where

$$
G(x)=F(x)+\frac{1}{2}-\int_{0}^{1} F(z) d z
$$

Also,

$$
|x-G(x)|=\lim _{n \rightarrow \infty}\left|x-G_{n}(x)\right| \leq \frac{1}{2} \quad \text { for all } x \in(0,1),
$$

so we deduce the extension $\tilde{G}$ of $G$ to $\mathbb{R}$ given by

$$
\tilde{G}(x):=G(x-k)+k \quad \text { if } k \leq x<k+1(k \in \mathbb{Z}),
$$

is the optimal map such that $\tilde{G}_{\#} \rho=\mathscr{L}_{\mathbb{T}^{1}}^{1}$ for any $\rho \sim \rho^{*}$ (note also that $\tilde{G}$ is nondecreasing as the limit of $\left.\tilde{G}_{n}\right)$. Finally, $P^{*}(x)=W * \rho^{*}(x)$ is differentiable a.e. in $(0,1)$ and we use (8.5) to see that

$$
\left(P^{*}\right)^{\prime}(x)=\lim _{n \rightarrow \infty} P_{n}^{\prime}(x)=x-\frac{1}{2}-F(x)+\int_{0}^{1} F(z) d z=x-G(x)
$$

for $\rho^{*}$ a.e. $x \in[0,1)$.

QED. 


\section{Bibliography}

[1] R. J. Duan, The Cauchy problem on the compressible two-fluids Euler-Maxwell Equations. Siam J. Math. Anal. Vol. 44, No. 1, pp.102-133.

[2] R. J. Duan, Global smooth flows for the compressible Euler-Maxwell System. The Relaxation case. Journal of Hyperbolic Differential Equations. (2011) Vol. 8, No. 2 , 375-413.

[3] C. Besse, J. Claudel, P. Degong, ET AL., A model hierarchy for ionospheric plasma modeling, Math. Models Methods Appl. Sci., 14 (2004), pp. 393-415.

[4] R. J. Duan, S. Ukai, and T. Yang, A combination of energy method and spectral analysis for study of equations of gas motion, Front. Math. China, 4 (2009), pp. 253-282.

[5] T. Kato, The Cauchy problem for quasi-linear symmetric hyperbolic systems, Arch. Ration. Mech. Anal., 58 (1975), pp. 181-205.

[6] M. E. Taylor, Partial differential equations, III. Basic Theory (Springer, New York, 1996).

[7] G. Q. Chen, J. W. Jerome and D. H. Wang, Compressible Euler-Maxwell equations, Trans. Th. Statist. Phys. 29 (2000) 311-331.

[8] Y. Ueda, S. Wang and S. Kawashima, Large global existence and asymptotic decay of solutions to the Euler-Maxwell system, SIAM J. Math. Anal. 44 (2012) 2002-2017.

[9] Y. Ueda and S. Kawashima, Decay property of regularity-loss type for the Euler- Maxwell system, Methods Appl. Anal. 18 (2011) 215-268.

[10] Y. J. Peng, S. Wang and Q. L. Gu, Relaxation limit and global existence of smooth solutions of compressible Euler-Maxwell equations, SIAM J. Math. Anal. 43 (2011) 944-970.

[11] Y. J. Peng and S. Wang, Convergence of compressible Euler-Maxwell equations to incompressible Euler equations, Commun. Part. Differential Equations 33 (2008) 349-376. 
[12] Y. J. Peng and S. Wang, Rigorous derivation of incompressible e-MHD equations from compressible Euler-Maxwell equations, SIAM J. Math. Anal. 40 (2008) 540-565.

[13] J. W. Yang and S. Wang, The diffusive relaxation limit of non-isentropic EulerMaxwell equations for plasmas, J. Math. Anal. Appl. 380 (2011) 343-353.

[14] J. W. Jerome, The Cauchy problem for compressible hydrodynamic-Maxwell systems: A local theory for smooth solutions, Differential Integral Equations 16 (2003) 1345-1368.

[15] Y. H. Feng, S. Wang and S. Kawashima, Global existence and asymptotic decay of solutions to non-isentropic Euler-Maxwell system. Vol. 24, No. 14(2014) 2851-2884. I.B. and A.T. would like to acknowledge partial support from the NSF award DMS-16 00272 (to A.T.). We would like to thank the anonymous referees for their suggestions to considerably improve this manuscript.

[16] N.D. Alikakos, G. Fusco, On the connection problem for potentials with several global minima, Indiana Univ. Math. J., 57 (2008), No. 4, pp. 18711906.

[17] L. Ambrosio, W. Gangbo, Hamiltonian ODE's in the Wasserstein space of probability measures, Comm. Pure Appl. Math. 61 (2008), No. 2, pp. 18-53.

[18] L. Ambrosio, N. Gigli, G. SaVArÉ, Gradient flows in metric spaces and the Wasserstein spaces of probability measures, Lectures in mathematics, E.T.H. Zurich, Birkhäuser, 2005.

[19] P. Antonopoulos, And P. SMyrnelis, On minimizers of the Hamiltonian system $u^{\prime \prime}=\nabla W(u)$ and on the existence of heteroclinic, homoclinic and periodic orbits, Indiana Univ. Math. J. 65 (2016), No. 5, pp. 1503-1524.

[20] V. ARnOld, Sur la geometrie differentielle de groupes de Lie de dimension infinie et ses applications a l'hydrodynamique des fluids parfaits, Ann. Inst. Fourier 16 (1966), pp. 316-361.

[21] Y. BRenIER, Optimal Transport, Convection, Magnetic Relaxation and Generalized Boussinesq equations, final version in JNLS (2009).

[22] J.D. Benamou, Y. Brenier, A Computational Fluid Mechanics solution to the Monge-Kantorovich mass transfer problem, Numer. Math. 84, no. 3 (2000), pp. 375-393.

[23] D. Cordero-Erausquin, Sur le transport de mesures périodiques, C. R. Acad. Sci. Paris, t. 239, Série 1, p. 199-202, (1999).

[24] B. Feng, R. Hu, A survey on homoclinic and heteroclinic orbits, Appl. Math. E-Notes 3 (2003), 16-37. 
[25] W. Gangbo, A. Tudorascu, A Weak KAM Theorem; from finite to infinite dimensions, Optimal Transportation, Geometry and Functional Inequalities, Edizioni della Normale (L. Ambrosio ed.) (2010), pp. 45-72.

[26] W. Gangbo, A. Tudorascu, Weak KAM theory on the Wasserstein torus with multi-dimensional underlying space, Comm. Pure Appl. Math. 67, No. 3 (2014), pp. 408-463.

[27] W. Gangbo, T. Nguyen And A. Tudorascu, Euler-Poisson systems as action-minimizing paths in the Wasserstein space, Archive for Rational Mechanics and Analysis. 192, No. 3 (2009), pp. 419-452.

[28] R. Jordan, D. Kinderlehrer, F. OtTo, The Variational Formulation of the Fokker-Plank Equation, SIAM J. Math. Anal. 29, No. 1 (1998), pp. 1-17.

[29] H. KIM, Hamiltonian systems and the calculus of differential forms on the Wasserstein space, PhD dissertation, 2008.

[30] A. Monteil, F. Santambrogio, Metric methods for heteroclinic connections, Math. Meth. Appl. Sci. 41 (2018), No. 3, pp. 1019-1024.

[31] A. Monteil, F. SAntambrogio, Heteroclinic connections in infinite dimensional spaces, arXiv:1709.02117 [math.AP].

[32] F. Отто, The geometry of dissipative evolution equations: the porous medium equation, Comm. PDE 26, No. 1-2 (2001), pp. 101-176.

[33] P. Rabinowitz, Periodic and heteroclinic orbits for a periodic Hamiltonian system, Ann. Inst. H. Poincaré C 6, No. 5 (1989), pp. 331-346.

[34] A. SCHNiRelman, Curvature and instability of flows of ideal incompressible fluids, BIRS workshop report, 2006.

[35] C. SouRDIS, The heteroclinic connection problem for general double-well potentials, Mediterranean J. Math.13 (2016), No. 6, pp. 4693-4710.

[36] P. Sternberg, A. ZunigA, On the heteroclinic connection problem for multiwell gradient systems, J.D.E. 261, no. 7 (2016), 3987-4007.

[37] C. Villani, Topics in Optimal Transportation, p. cm. - (Graduate Studies in Math. ISSN 1065-7339; V.58) QA402 .6 . V56 (2003). 